\title{
Identification of potential and novel target genes in pituitary prolactinoma by bioinformatics analysis
}

Vikrant Ghatnatti ${ }^{1}$, Basavaraj Vastrad ${ }^{2}$, Swetha Patil ${ }^{3}$, Chanabasayya Vastrad ${ }^{4 *}$, Iranna Kotturshetti ${ }^{5}$

1. Dept of Endocrinology/Medicine, J N Medical College, Belagavi 590010, Karnataka, India.

2. Department of Biochemistry, Basaveshwar College of Pharmacy, Gadag, Karnataka 582103, India.

3. Dept of Obstetrics and Gynaecology, J N Medical college, Belagavi 590010, Karnataka, India.

4. Biostatistics and Bioinformatics, Chanabasava Nilaya, Bharthinagar, Dharwad 580001 , Karanataka, India.

5. Department of Ayurveda, Rajiv Gandhi Education Society`s Ayurvedic Medical College, Ron 562209, Karanataka, India.

* Chanabasayya Vastrad

channu.vastrad@gmail.com

Ph: +919480073398

Chanabasava Nilaya, Bharthinagar,

Dharwad 580001 , Karanataka, India

\section{Abstract}


Pituitary prolactinoma is one of the most complicated and fatally pathogenic pituitary adenomas. Therefore, there is an urgent need to improve our understanding of the underlying molecular mechanism that drives the initiation, progression, and metastasis of pituitary prolactinoma. The aim of the present study was to identify the key genes and signaling pathways associated with pituitary prolactinoma using bioinformatics analysis. Transcriptome microarray dataset GSE119063 was acquired from Gene Expression Omnibus datasets, which included 5 pituitary prolactinoma samples and 4 normal pituitaries samples. We screened differentially expressed genes (DEGs) with limma and investigated their biological function by pathway and Gene Ontology (GO) enrichment analysis. A protein-protein interaction (PPI) network of the up and down DEGs were constructed and analyzed by HIPPIE and Cytoscape software. Module analyses were performed. In addition, a target gene - miRNA network and target gene - TF network of the up and down DEGs were constructed by NetworkAnalyst and Cytoscape software. The set of DEGs exhibited an intersection consisting of 989 genes (461 up-regulated and 528 down-regulated), which may be associated with pituitary prolactinoma. Pathway enrichment analysis showed that the 989 DEGs were significantly enriched in the retinoate biosynthesis II, signaling pathways regulating pluripotency of stem cells, ALK2 signaling events, vitamin D3 biosynthesis, cell cycle and aurora B signaling. Gene Ontology (GO) enrichment analysis also showed that sensory organ morphogenesis, extracellular matrix, hormone activity, nuclear division, condensed chromosome and microtubule binding. In the PPI network and modules, SOX2, PRSS45, CLTC, PLK1, B4GALT6, RUNX1 and GTSE1 were considered as hub genes. In the target gene miRNA network and target gene - TF network, LINC00598, SOX4, IRX1 and UNC13A were considered as hub genes. Using integrated bioinformatics analysis, we identified candidate genes in pituitary prolactinoma, which may improve our understanding of the mechanisms of the pathogenesis and integration; genes may be therapeutic targets and prognostic markers for pituitary prolactinoma.

Key words: pituitary prolactinoma; differentially expressed genes; gene ontology; pathway enrichment analysis; protein-protein interactions

\section{Introduction}


Prolactinoma is named as prolactin secreting pituitary adenoma seen more frequently in women and is characterized by irregular menstrual, erectile dysfunction, eye problems and loss of sexual function [1]. Its typical signs and symptoms include amenorrhoea, glactorrhoea, headache, anaemia and hypertension [2]. Prolactinoma involves $20.7 \%$ among other pituitary adenomas [3]. Although treatment methods, including surgery [4], chemotherapy [5] and radiotherapy [6] are improving, overall survival rate remains lower. Consequently, elucidating the molecular mechanism associated in the proliferation of pituitary prolactinoma is essential for the improvement of efficacious diagnosis and treatment strategies.

Presently, a wealth of previous studies has been enforced to advance a better understanding of the molecular mechanisms of pituitary prolactinoma. One study showed that allelic loss of DRD2 is responsible for development of pituitary prolactinoma [7]. Elevated expression of HMGA1 and HMGA2 are diagnosed with pituitary prolactinoma [8-9]. BMP4 is associated with SMAD4 and ER in development of pituitary prolactinoma [10]. FGF4 is responsible for improvement of pituitary prolactinoma through invasion and cell proliferation [11]. Alteration in oncogene GNAS is important for improvement of pituitary prolactinoma [12]. Stimulation of Raf/MEK/ERK and PI3K/Akt/mTOR signaling pathway is liable for development of prolactinoma [13]. Abnormal expression of CTNNB1 is diagnosed with prolactinoma [14]. Epigenetic inactivation of CDKN2A is responsible for advancement of prolactinoma [15]. AIP play key role in pathogenesis of pituitary prolactinoma [16]. Hence, searching for specific and sensitive molecular marker as well as some core genes or proteins as therapeutic target will benefit the diagnosis and treatment of pituitary prolactinoma.

At current study, microarray analysis has been applied as a very critical tool for medical research [17]. In this analysis, we chose GSE119063 dataset from Gene Expression Omnibus (GEO) (http:// www.ncbi.nlm.nih.gov/geo/) and used limma bioconductor package to find the differentially expressed genes (DEGs). Subsequently, we made pathway enrichment and gene ontology (biological process (BP), molecular function (MF), cellular component (CC) analyses were performed. In addition, we made PPI network of the DEGs and selected core genes with a high degree of connectivity, high betweenness centrality, high stress centrality, high closeness centrality and low Clustering coefficient, and modules analysis were 
performed. Moreover, miRNA-target gene regulatory network and TF-target gene regulatory network were constructed and analyzed. Briefly, this study would provide novel targets for diagnosis and treatment of pituitary prolactinoma.

\section{Results}

\section{Identification of DEGs}

After data preprocessing, the raw data of nine samples is proved to be eligible (Fig. 1). The GSE119063 expression profile data from GEO was investigated to screen for DEGs between the experimental and control groups. Under the threshold of FDR $<0.05$, and fold change $\geq 0.93$ for up regulated gene and fold change $\geq-0.29$ for down regulated gene. Comparison of prolactinoma with normal pituitaries identified total of 989 DEGs, including 461 up regulated genes and 528 down regulated genes, were revealed (Table 1). A corresponding heat map is shown in Fig. 2 and Fig 3. All the DEGs were presented by volcano plot in the study (Fig. 4).

\section{Pathway enrichment analysis of DEGs}

Several significant enriched pathways are acquired through BIOCYC, KEGG, PID, REACTOME, GenMAPP, MSigDB C2 BIOCARTA, PantherDB, Pathway Ontology and SMPDB pathway enrichment analysis (Table 2 and Table 3). The top enriched pathways for up regulated genes included retinoate biosynthesis II, retinoate biosynthesis I, signaling pathways regulating pluripotency of stem cells, neuroactive ligand-receptor interaction, ALK2 signaling events, BMP receptor signaling, peptide hormone biosynthesis, glycoprotein hormones, tyrosine metabolism, androgen and estrogen metabolism, ensemble of genes encoding extracellular matrix and extracellular matrix-associated proteins, genes encoding secreted soluble factors, adenine and hypoxanthine salvage pathway, 5Hydroxytryptamine biosynthesis, melanocortin system, androgen and estrogen metabolic, tryptophan metabolism and xanthine dehydrogenase deficiency (Xanthinuria). Meanwhile, down regulated DEGs strikingly enriched in vitamin D3 biosynthesis, cell cycle, pancreatic secretion, aurora B signaling, FOXM1 transcription factor network, mitotic prometaphase, resolution of sister chromatid cohesion, role of ran in mitotic spindle regulation, Eph kinases and ephrins support platelet aggregation, inflammation mediated by chemokine and cytokine signaling 
pathway, O-Glycans biosynthetic, ganglioside biosynthetic, eptifibatide pathway and ticlopidine pathway.

\section{Gene Ontology (GO) enrichment analysis of DEGs}

All significant DEGs were divided into up regulated genes and down regulated genes. GO categories analyses are conducted for these 2 lists of genes, respectively. Results of GO categories are presented by 3 functional groups, which are group BP, CC, and MF (Table 4 and Table 5). In group BP, up and down regulated DEGs are significantly enriched in sensory organ morphogenesis, embryonic organ morphogenesis, nuclear division and organelle fission. For group $\mathrm{CC}$, up and down regulated DEGs mainly enriched in extracellular matrix and extracellular space, condensed chromosome and kinetochore. In addition, GO results of group MF showed that up and down regulated DEGs mainly enriched in hormone activity, signaling receptor binding, microtubule binding and microtubule motor activity.

\section{PPI network construction and topology analysis}

PPI networks were constructed on the basis of HIPPIE online tool. We also analyzed the network properties such as node degree, betweenness centrality, stress centrality, closeness centrality and cluster coefficient. The PPI network for up regulated DEGs is shown in Fig. 5, which has 4005 nodes and 5562 interactions. The top 5 nodes with greater degrees are listed in Table 6, including SOX2 (degree = 355), KRT40 $($ degree $=313)$, SMAD9 $($ degree $=114)$, AMOT $($ degree $=111)$ and DPPA4 (degree $=105$ ). $\mathrm{R}$ square and correlation coefficient are 0.776 and 0.966, respectively (Fig. 7A). Top 5 up regulated genes with high betweenness centrality are SOX2 (betweeness $=0.20715558$ ), KRT40 (betweeness = 0.15884752), SMAD9 (betweeness $=0.07865032$ ), AMOT (betweeness $=$ 0.06814966 ) and TRIM29 (betweeness $=0.0613444$ ) shown in Table 6. R square and correlation coefficient are 0.474 and 0.098, respectively (Fig. 8A). Top 5 up regulated high stress genes are SOX2 (stress $=37524600$ ), KRT40 (stress = 22565808), AMOT (stress $=13889668$ ), C6orf141 (stress $=9207512$ ) and TRIM29 (stress $=9041322$ ) shown in Table 6. R square and correlation coefficient are 0.088 and 0.072 , respectively (Fig. 8B). Top 5 up regulated gene with high closeness centrality are SOX2 (closeness $=0.31817447$ ), KRT40 (closeness = 
0.30482897), SMAD9 (closeness $=0.30309326)$, TRIM29 (closeness $=$ 0.2975525 ) and FGFR2 (closeness $=0.29117386$ ) shown in Table 6. R square and correlation coefficient are 0.036 and 0.082 , respectively (Fig. 8C). Top 5 up regulated gene with low clustering coefficient are PRSS45 (clustering coefficient = 0 ), CARTPT (clustering coefficient $=0$ ), TAC4 (clustering coefficient $=0$ ), CT45A1 (clustering coefficient $=0$ ) and PON3 (clustering coefficient $=0$ ) shown in Table 6. R square and correlation coefficient are 0.616 and 0.882 , respectively (Fig. 8D).

The PPI network for down regulated DEGs is shown in Fig. 6, which has 5441 nodes and 9866 interactions. The top 5 nodes with grater degrees are listed in Table 6, including CLTC (degree = 333), PLK1 (degree = 276), DHX15 (degree = 208), GTSE1 (degree $=141$ ) and DISC1 (degree $=138$ ). $\mathrm{R}$ square and correlation coefficient are 0.756 and 0.955 , respectively (Fig. 7B). Top 5 down regulated genes with high betweenness centrality are CLTC (betweeness = 0.17279707), PLK1 (betweeness $=0.16393694$ ), DHX15 (betweeness = 0.09387272 ), KIF11 (betweeness $=0.05972209$ ) and ATP2B2 (betweeness = 0.0557287 ) shown in Table 6 . $\mathrm{R}$ square and correlation coefficient are 0.596 and 0.119, respectively (Fig. 9A). Top 5 down regulated genes with high stress genes are CLTC (stress = 59969246), DHX15 (stress = 39966314), PLK1 (stress = 39488508), ATP2B3 (stress = 23062588) and KIF11 (stress = 17181634) shown in Table 6. R square and correlation coefficient are 0.371 and 0.004 , respectively (Fig. 9B). Top 5 down regulated genes with high closeness centrality are PLK1 $($ closeness $=0.35244041)$, CLTC $($ closeness $=0.34583256)$, ATP2B4 $($ closeness $=$ 0.33762233 ), ATP2B8 (closeness $=0.32332523$ ) and DHX15 (closeness = 0.32084733 ) shown in Table 6. R square and correlation coefficient are 0.081 and 0.144 , respectively (Fig. 9C). Top 5 up regulated gene with low clustering coefficient are B4GALT6 (clustering coefficient $=0$ ), ZNF160 (clustering coefficient $=0$ ), HIGD1B (clustering coefficient $=0$ ), CCL3L3 (clustering coefficient $=0$ ) and C20orf203 (clustering coefficient $=0$ ) shown in Table $6 . \mathrm{R}$ square and correlation coefficient are 0.569 and 0.860, respectively (Fig. 9D).

\section{Module analysis}

A total of 332 modules are identified in up regulated PPI network, among which the best are module 1, module 2, module 3 and module 10 (Fig. 10). Module 1 is 
composed of 17 nodes and 33 edges. The hub proteins in this module such as RUNX1 (degree $=79)$ and SOX2 (degree $=355)$ are involved in module 1. Module 2 is composed of 11 nodes and 23 edges. The hub proteins in this module such as FGFR2 (degree $=93)$, FGF1 (degree $=27)$ and FGFR3 (degree $=49)$ are involved in module 2. Module 3 is composed of 11 nodes and 21 edges. The hub proteins in this module such as S100B (degree $=33$ ) and S100A1 (degree $=23$ ) are involved in module 3. Module 10 is composed of 5 nodes and 8 edges. The hub proteins in this module such as SMAD9 (degree $=114)$ and EVC2 (degree $=86)$ are involved in module 10 .

A total of 425 modules are identified in down regulated PPI network, among which the best are module 1, module 5, module 8 and module 12 (Fig.11). Module 1 is composed of 80 nodes and 157 edges. The hub proteins in this module such as CLTC (degree $=333$ ) and GTSE1 (degree $=141$ ) are involved in module 1. Module 5 is composed of 15 nodes and 43 edges. The hub proteins in this module such as NUF2 (degree $=44)$, BUB1 (degree $=95)$, SPC24 (degree $=41$ ) and SPC25 (degree $=35)$ are involved in module 5. Module 8 is composed of 11 nodes and 23 edges. The hub proteins in this module such as CKS2 (degree $=36$ ), CCNB2 (degree $=36$ ) and CCNA1 (degree $=83$ ) are involved in module 8 . Module 12 is composed of 9 nodes and 17 edges. The hub proteins in this module such as KIF18A (Degree 27), FOXM1 (degree $=80)$ and PRC1 (degree $=29)$ are involved in module 12 .

\section{Construction of the target gene - miRNA network}

The miRNAs that may control the DEGs are diagnosed based on the up and down regulation expressions (Fig. 12 and Fig. 13). Top 5 up regulated targeted genes such as LINC00598 regulated by 209 miRNAs, CNKSR3 regulated by 138 miRNAs, PMAIP1 regulated by 128 miRNAs, TRIM71 regulated by 104 miRNAs and FAM83F regulated by 94 miRNAs are given in Table 7. Top 5 down regulated targeted genes such as SOX4 regulated by 160 miRNAs, ZMAT3 regulated by 145 miRNAs, PTP4A1 regulated by 132 miRNAs, RAD51 regulated by 113 miRNAs and DAZAP2 regulated by 109 miRNAs are given in Table 7.

\section{Construction of the target gene - TF network}


The TFs for target up and down regulated genes are shown in Fig. 14 and Fig. 15, respectively. Top 5 up regulated targeted genes such as IRX1 regulated by 73 TFs, CACNA2D3 regulated by 30 TFs, VSNL1 regulated by 29 TFs, BMP7 regulated by 28 TFs and DACT2 regulated by 27 TFs are given in Table 8 . Top 5 down regulated targeted genes such as UNC13A regulated by $43 \mathrm{TFs}$, CCNF regulated by 35 TFs, BDNF regulated by 34 TFs, POU3F4 regulated by 34 TFs and AKAP3 regulated by 31 TFs are given in Table 8 .

\section{Discussion}

A molecular understanding of pituitary prolactinoma is particularly essential to eventually improve effective approaches for its control, treatment, and prevention. In the present study, important candidate genes of pituitary prolactinoma were identified by bioinformatics analysis. We extracted the gene expression data from GSE119063 and obtained 461 up regulated and 528 down regulated DEGs in pituitary prolactinoma. SALL1 action as a tumor suppressor and epigenetic inactivation of this gene is responsible for development of cancer [18], but association of this gene still not reported in pituitary prolactinoma. Wang et al [19] showed that expression of homeodomain transcription factor NKX2-2 is linked with neuro endocrine tumor. High expression of pleiotropic signaling molecule BMP7 controls proliferation, migration, and invasion of cancer cells [20], but this gene may be linked with pathogenesis of pituitary prolactinoma. Cipriano et al [21] identified that oncogene FAM83F is associated in stimulating CRAF/MAPK signaling pathway and driving epithelial cell transformation in cancer, but involvement of this gene may responsible for advancement of pituitary prolactinoma. Oncogene GRHL2 is a transcriptional controller of proliferation and differentiation in epithelial cells, both during progression and tumor development, but this gene still not reported in pituitary prolactinoma [22]. TRIM24 play key role in proliferation and invasion of cancer cell [23], but high expression of this gene may be responsible for development of pituitary prolactinoma. Kaistha et al [24] describe that high expression of dual specificity kinase TTK shows proliferative potential of cancer, but this gene may be linked with pathogenesis of pituitary prolactinoma. Over expression of TOP2A is responsible for improvement of cancer [25], but this gene may associate with pituitary prolactinoma. Potential role KIF18A is associated with abnormal cell division, checkpoint activation and tumor formation [26], but this gene may be responsible for advancement pituitary 
prolactinoma. Over expression of microtubule associated protein TPX2 stimulates the cell cycle kinase aurora A and strongly correlates with chromosomal instability in cancer [27], but this gene may be associated with pathogenesis of pituitary prolactinoma. Genes such as FGFR2 [28], SOX2 [29], POMC [30], FSHB [31], EFEMP1 [32], SFRP2 [33], CSH2 [34], IHH [35], GH1 [36], PTTG2 [37], CCNB2 [38], RACGAP1 [39], CXCR2 [40], CXCR3 [41], FOXL2 [42], RUNX2 [43], TF [44], CCK [45], DPPA4 [46], RUNX1 [43] and BDNF [47] are associated with pituitary prolactinoma.

In present study retinoate biosynthesis II is the most significant BIOCYC pathway for up-regulated genes. XDH and RBP4 are novel biomarkers for pathogenesis of pituitary prolactinoma. Signaling pathways regulating pluripotency of stem cells is the most significant KEGG pathway for up-regulated genes. Alteration in FGFR3 is diagnosed with bladder cancer [48], but this gene may be important for development of pituitary prolactinoma. WNT5A improves cancer cell invasion and proliferation through receptor-mediated endocytosis dependent and independent mechanisms [49], but this gene may be diagnosed with pituitary prolactinoma. ID4 is potential tumor suppressor and epigenetic inactivation of this gene linked with prostate cancer [50], but this gene may be liable for advancement of pituitary prolactinoma. High expression of INHBA improves cell proliferation and is associated with progression of lung adenocarcinoma [51], but this gene may be involve in pathogenesis of pituitary prolactinoma. PAX6 prevents cancer cell proliferation and invasion and epigenetic silencing of this is diagnosed with breast cancer [52], but this gene may be culpable for progression of pituitary prolactinoma. LEFTY2 and SMAD9 are novel biomarkers for pathogenesis of pituitary prolactinoma. ALK2 signaling events are the most significant PID pathway for up regulated genes. Peptide hormone biosynthesis is the most significant REACTOME pathway for up-regulated genes. CGB3 and TSHB are novel biomarkers for pathogenesis of pituitary prolactinoma. Tyrosine metabolism was the most significant GenMAPP pathway for up-regulated genes. Duan et al [53] showed that ALDH1A3 belongs to the member of aldehyde dehydrogenase (ALDH) superfamily and play a regulatory role in the initiation and development of cancer via the clearance of aldehydes and the synthesizes of retinoic acid, but this gene may be linked with pathogenesis of pituitary prolactinoma. PNMT and AOX1 are novel biomarkers for pathogenesis of pituitary prolactinoma. Ensemble 
of genes encoding extracellular matrix and extracellular matrix-associated proteins is the most significant MSigDB C2 BIOCARTA pathway for up-regulated genes. S100A14 is belongs to a member of the S100 protein family and actions as a modulator of HER2 signaling pathway in breast cancer [54], but this gene may be responsible for development of pituitary prolactinoma. DeRycke et al [55] found that high expression of calcium binding protein S100A1 is identified with ovarian cancer, but this gene may be important for improvement of pituitary prolactinoma. Harpio and Einarsson [56] identified that high level of S100B is answerable for development of melanoma, but this gene may be identified with pituitary prolactinoma. Methylation and silencing of large modular extracellular matrix glycoprotein FBN2 play an essential role in carcinogenesis, invasion and metastasis of non-small cell lung cancer [57], but this gene may be involved in pathogenesis of pituitary prolactinoma. Yoshimura et al [58] reported that FGF1 is an inducer of angiogenesis in breast cancer, but this gene may play a role in the pathogenesis of pituitary prolactinoma. SLIT1 is tumor suppressor gene and epigenetic suppression of this gene is associated with cancer [59], but this silenced gene may be culpable for development pituitary prolactinoma. ADAMTS18 and CHRDL1 are putative tumor suppressive genes and epigenetic silencing of these genes diagnosed with gastric, colorectal and pancreatic cancers [60-61], but inactivation of these genes may be link with pituitary prolactinoma. VIT, MFAP4, FCN2, SBSPON, SPON1, ITIH6, COL4A6, COL8A2, FRAS1, COL13A1, ITLN1, ADAM32, CSH1, CSHL1, SLPI, IFNE, MEGF11, LAMA1, ITIH2, GH2, COL21A1, CLEC9A, NTF4, OGN, RSPO3, MEGF6, CBLN2 and LGI1 are novel biomarkers for pathogenesis of pituitary prolactinoma. Adenine and hypoxanthine salvage pathway is the most significant PantherDB pathway for up-regulated genes. Melanocortin system is the most significant Pathway Ontology for upregulated genes. PCSK2 is novel biomarkers for pathogenesis of pituitary prolactinoma. Tryptophan metabolism is the most significant PantherDB pathway for up-regulated genes. TDO2 and TPH1 are novel biomarkers for pathogenesis of pituitary prolactinoma. Vitamin D3 biosynthesis is the most significant BIOCYC pathway for down-regulated genes. Polymorphisms of CYP27B1 is modulate vitamin $\mathrm{D}$ metabolism in colon cancer [62], but this polymorphic gene may be responsible for improvement of pituitary prolactinoma. Cell cycle is the most significant KEGG pathway for down-regulated genes. High expression of PLK1 is linked with cancer cell proliferation [63], but this may be gene is liable for 
development pituitary prolactinoma. High expression of mitotic spindle checkpoint gene BUB1 is linked with gastric cancer cell proliferation [64], but this gene may be important in pathogenesis of pituitary prolactinoma. Epigenetic inactivation of CCNA1 is diagnosed with cervical cancer [65], but silencing of this gene may be responsible for advancement of pituitary prolactinoma. CDC45 is novel biomarker for pathogenesis of pituitary prolactinoma. Aurora B signaling is the most significant PID pathway for down-regulated genes. KIF23 plays as both a controller of cytokinesis and a motor enzyme of microtubule and this gene is over expressed in lung cancer [66], but this gene may be play crucial role in pituitary prolactinoma. BIRC5 play key role in cell division and proliferation and by inhibiting apoptosis in hepatocellular carcinoma [67], but this gene may be link with pituitary prolactinoma. NCAPG improves hepatocellular cancer cell proliferation and migration [68], but this gene may be diagnosed with pituitary prolactinoma. Nie et al [69] suggest that STMN1 play essential role in the control of cellular division and proliferation in non-small cell lung cancer, but this gene may be involved in pathogenesis of pituitary prolactinoma. High expression of KIF20A is associated with pancreatic cancer [70], but this gene may be play key role in pituitary prolactinoma. CENPA and KLHL13 are novel biomarkers for pathogenesis of pituitary prolactinoma. Mitotic prometaphase is the most significant REACTOME pathway for down-regulated genes. High expression of SPC24 and SPC25 are associated with genomic instability and disrupted regulation of cell cycle in lung cancer, but this gene may be responsible for progression of pituitary prolactinoma [71]. Over expression cell cycle molecular marker CENPH pay key role in gastric carcinoma cell proliferation and gastric carcinoma cell survival as well as procancer role [72], but this gene may be involved in pituitary prolactinoma. Over expression of NUF2 activates tumor growth and inhibits cell apoptosis [73], but this gene may be liable for pathogenesis of pituitary prolactinoma. CENPM, CENPI, CDCA5 and CENPU are novel biomarkers for pathogenesis of pituitary prolactinoma. Role of Ran in mitotic spindle is the most significant MSigDB C2 BIOCARTA pathway for down-regulated genes. RCC1 and KIF15 are novel biomarkers for pathogenesis of pituitary prolactinoma. Inflammation mediated by chemokine and cytokine signaling pathway is the most significant PantherDB pathway for down-regulated genes. PF4-active platelet accumulation in cancer is crucial because platelets can modulate cancer cells and the cancer microenvironment to stimulate lung Cancer outgrowth [74], but this 
gene may be answerable for development of pituitary prolactinoma. VWF, CCL3L3, CASK and PLCD4 are novel biomarkers for pathogenesis of pituitary prolactinoma. O-glycans biosynthetic is the most significant Pathway Ontology for down-regulated genes. B4GALT6 is novel biomarker for pathogenesis of pituitary prolactinoma. Eptifibatide pathway is the most significant SMPDB pathway for down-regulated genes. RYR2 is novel biomarker for pathogenesis of pituitary prolactinoma.

In present study, sensory organ morphogenesis is the most significant GO BP term for up-regulated genes. Epigenetic inactivation of tumor suppressor genes EYA4 and GAS1are associated with lung cancer and papillary thyroid carcinoma development, but silencing of these genes may associate with pituitary prolactinoma [75-76]. Expression of SOX9 enhances colorectal cancer cell invasion and migration [77], but this gene may link with pathogenesis of pituitary prolactinoma. ROR2 play a key role as an important mediator of the Wnt signaling pathway in colorectal cancer [78], but this gene may be involves in pathogenesis of pituitary prolactinoma through activation of Wnt signaling pathway. CLIC5, TMIE, CLRN1, PRRX2, SDK2, CELSR1, STRA6, CALB1 and VANGL2 are novel biomarkers for pathogenesis of pituitary prolactinoma. Extracellular matrix is the most significant GO CC term for up-regulated genes. FLRT2, CPXM2, LAD1 and TGFBR3 are novel biomarkers for pathogenesis of pituitary prolactinoma. Hormone activity is the most significant GO MF term for upregulated genes. CARTPT, GPHA2 and GAL are novel biomarkers for pathogenesis of pituitary prolactinoma. Nuclear division is the most significant GO BP term for down-regulated genes. Elevated expression of NUSAP1 may improve cancer progression by increasing proliferation and invasion of prostate cancer cells [79], but this gene may be responsible for progression of pituitary prolactinoma. High expression of cell cycle regulatory protein CKS2 is culpable for advancement of gastric cancer [80], but this gene may be link with pathogenesis of pituitary prolactinoma. PBK is likely to play a crucial role in cell division and cytokinesis in breast cancer [81], but this gene may be liable for improvement of pituitary prolactinoma. Inappropriate expression of NEK2 interfere with mitotic processes results in breast cancer development [82], but this gene may be play key role in pathogenesis of pituitary prolactinoma. High expression of oncogene KIF14 may play crucial role in cancer development [83], 
but this gene may be associates with pituitary prolactinoma. Wong et al [84] and Loveday et al [85] demonstrated that modification of normal DNA repair function of RAD51 and RAD51D may lead to genomic instabilities that eventually contribute to breast and ovarian cancer development, but gene may be identify with pituitary prolactinoma. CCNG2 deeply involved in pancreatic cancer cell proliferation, invasion, chemoresistance, and differentiation [86], but this gene may be responsible for important for the pathogenesis of pituitary prolactinoma. FANCA, FANCD2, CLTC, MTBP, HAUS6, DLGAP5, RAD21L1, PTTG3P, KIF11, PRC1, EDN1 and CCNF are novel biomarkers for pathogenesis of pituitary prolactinoma. Condensed chromosome is the most significant GO CC term for down-regulated genes. HJURP is novel biomarker for pathogenesis of pituitary prolactinoma. Microtubule binding is the most significant GO MF term for downregulated genes. KIF21B and KIF26A are novel biomarkers for pathogenesis of pituitary prolactinoma.

In present study, SOX2, KRT40, SMAD9, AMOT and DPPA4 were identified as hub proteins (up regulated DEGs) in the PPI network. AMOT control of the Hippo/LATS pathway in the processes of cell proliferation, motility, and differentiation in cancer [87], but this may be linked with pituitary prolactinoma. KRT40 is novel biomarkers for pathogenesis of pituitary prolactinoma. SOX2, KRT40, SMAD9, AMOT and TRIM29 are the hub proteins (up regulated DEGs) with highest betweenness centrality in the PPI network. High expression of TRIM29 is important in differentiation, proliferation, and development of gastric cancer [88], but this gene may be responsible for progression of pituitary prolactinoma. SOX2, KRT40, AMOT, C6orf141 and TRIM29 are the hub proteins (up regulated DEGs) with highest stress centrality in the PPI network. C6orf141 is novel biomarkers for pathogenesis of pituitary prolactinoma. SOX2, KRT40, SMAD9, TRIM29 and FGFR2 are the hub proteins (up regulated DEGs) with highest closeness centrality in the PPI network. PRSS45, CARTPT, TAC4, CT45A1 and PON3 are the hub proteins (up regulated DEGs) with lowest clustering coefficient in the PPI network. Shang et al [89] reported that protooncogene CT45A1 play key role cancer cell invasion, but this gene may be link with pituitary prolactinoma. PRSS45, TAC4 and PON3 are novel biomarkers for pathogenesis of pituitary prolactinoma. CLTC, PLK1, DHX15, GTSE1 and DISC1 are identified as hub proteins (down regulated DEGs) in the PPI network. 
DHX15 play key role in cancer through activating AR activity through Siah2mediated ubiquitination independent of its ATPase activity [90], but this gene may be liable for development of pituitary prolactinoma. Subhash et al [91] shown that GTSE1 is up-regulated in gastric cancer through DNA damage activated transactivation of p53 is inhibited, thus affecting p53 activated apoptosis, but this gene may be answerable for development of pituitary prolactinoma. DISC1 is novel biomarker for pathogenesis of pituitary prolactinoma. CLTC, PLK1, DHX15, KIF11 and ATP2B2 are the hub proteins with highest betweenness centrality in the PPI network for down regulated DEGs. ATP2B2 is novel biomarker for pathogenesis of pituitary prolactinoma. CLTC, DHX15, PLK1, ATP2B3 and KIF11 are the hub proteins with highest stress centrality in the PPI network for down regulated DEGs. ATP2B3 is novel biomarker for pathogenesis of pituitary prolactinoma. PLK1, CLTC, ATP2B4, ATP2B8 and DHX15 are the hub proteins with highest closeness centrality in the PPI network for down regulated DEGs. ATP2B4 and ATP2B8 are novel biomarkers for pathogenesis of pituitary prolactinoma. B4GALT6, ZNF160, HIGD1B, CCL3L3 and C20orf203 are the hub proteins with lowest clustering coefficient in the PPI network for down regulated DEGs. ZNF160, HIGD1B and C20orf203 are novel biomarkers for pathogenesis of pituitary prolactinoma.

Modules are extracted from the PPI network for up and down regulated DEGs. RUNX1, SOX2, FGFR2, FGF1, FGFR3, S100B, S100A1, SMAD9 and EVC2 are the hub proteins (up regulated DEGs with high degree) in all four modules in the PPI network. EVC2 is novel biomarker for pathogenesis of pituitary prolactinoma. CLTC, GTSE1, NUF2, BUB1, SPC24, SPC25, CKS2, CCNB2, CCNA1, KIF18A, FOXM1 and PRC1 are the hub proteins (down regulated DEGs with high degree) in all four modules in the PPI network. FOXM1 play roles in cancer related processes, such as invasion and metastasis [92], but this may be link with pathogenesis of pituitary prolactinoma.

LINC00598, CNKSR3, PMAIP1, TRIM71 and FAM83F are identified as up regulated target genes with high degree of connectivity in target gene - miRNAregulatory network. LINC00598, CNKSR3, PMAIP1 and TRIM71 are novel biomarkers for pathogenesis of pituitary prolactinoma. SOX4, ZMAT3, PTP4A1, RAD51 and DAZAP2 are identified as down regulated target genes with high degree of connectivity in target gene-miRNA regulatory network. Oncogene SOX4 
plays an essential role in the stimulation of and response to developmental pathways, such as Wnt, Notch, Hedgehog, and TGF $\beta$ in prostate cancer [93], but this gene may be associates with pituitary prolactinoma. PTP4A1 play key role in cancer cell growth and invasion of breast cancer cells [94], but this gene may be responsible for improvement of pituitary prolactinoma. ZMAT3 and DAZAP2 are novel biomarkers for pathogenesis of pituitary prolactinoma.

IRX1, CACNA2D3, VSNL1, BMP7 and DACT2 are identified as up regulated target gene with high degree of connectivity in TFs-target gene regulatory network. CACNA2D3 and DACT2 are tumor suppressor genes and epigenetic inactivation of this gene is linked with gastric and lung cancer [95-96], but suppression of these genes may be identify with pituitary prolactinoma. IRX1 and VSNL1 are novel biomarkers for pathogenesis of pituitary prolactinoma. UNC13A, CCNF, BDNF, POU3F4 and AKAP3 are identified as down regulated target genes with high degree of connectivity in TFs- target gene regulatory network. UNC13A, POU3F4 and AKAP3 are novel biomarkers for pathogenesis of pituitary prolactinoma.

\section{Methods}

\section{Microarray Data}

We chose a gene expression profile of GSE119063 from GEO database. GSE119063 was based on the Agilent GPL13607 platform (Agilent-028004 SurePrint G3 Human GE 8x60K Microarray (Feature Number version)). The GSE119063 dataset included 9 samples, containing 5 pituitary prolactinoma samples and 4 normal pituitaries samples. Besides, we downloaded the Series Matrix File of GSE119063 from GEO database.

\section{Data preprocessing}

The raw data used in this study were downloaded. The raw GSE119063 data was preprocessed by the Limma package (http://www.bioconductor.org/packages/release/bioc/html/limma.html) [97] in Bioconductor. The data preprocessing included background correction and quantile normalization. Probe identities (IDs) are mapped to gene IDs using the corresponding platform files. 


\section{Identification of DEGs}

The Limma package was subsequently used for identifying DEGs. $\mathrm{p}<0.05$ and absolute fold change $\geq 0.93$ for up regulated gene and fold change $\geq-0.29$ for down regulated gene [98] were considered as the cutoff values for DEG screening using the Benjamini \& Hochberg procedure. R software was used to produce heat maps of common significant differentially expressed genes between pituitary prolactinoma samples and normal pituitaries samples. Genes are ordered according to the fold change in the expression values. This information was presented as a heat map and a volcano plot.

\section{Pathway enrichment analysis of DEGs}

BIOCYC (https://biocyc.org/) [99], Kyoto Encyclopedia of Genes and Genomes (KEGG) (http://www.genome.jp/kegg/pathway.html) [100], Pathway Interaction Database

(https://wiki.nci.nih.gov/pages/viewpage.action?pageId=315491760) REACTOME (https://reactome.org/) [102], GenMAPP (http://www.genmapp.org/) [103], MSigDB

$\mathrm{C} 2$

BIOCARTA (http://software.broadinstitute.org/gsea/msigdb/collections.jsp) [104], PantherDB (http://www.pantherdb.org/) [105], Pathway Ontology (http://www.obofoundry.org/ontology/pw.html) [106] and Small Molecule Pathway Database (SMPDB) (http://smpdb.ca/) [107] were a collection of databases which helps to handle genomes, biological pathways, diseases, chemical substances, and drugs. ToppGene (https://toppgene.cchmc.org/enrichment.jsp) is a web-based online bioinformatics resource that aims to provide tools for the functional interpretation of large lists of genes or proteins [108]. $p$ value $<0.05$ is regarded as the cutoff criterion. We could visualize the pathways among those DEGs using ToppGene.

\section{Gene Ontology (GO) enrichment analysis of DEGs}

Gene ontology (GO) (http://www.geneontology.org/) enrichment analysis served as a useful approach to annotate genes and gene products and also analyze characteristic biological attributing to high-throughput genome or transcriptome data [109]. ToppGene (https://toppgene.cchmc.org/enrichment.jsp) is a web-based online bioinformatics resource that aims to provide tools for the functional 
interpretation of large lists of genes or proteins [108]. $\mathrm{P}$ value $<0.05$ is regarded as the cutoff criterion. We could visualize the core biological process (BP), molecular function (MF) and cellular component (CC) among those DEGs using ToppGene.

\section{PPI network construction and topology analysis}

The Human Integrated Protein-Protein Interaction rEference (HIPPIE) (http://cbdm.uni-mainz.de/hippie/) is an online tool providing experimental and predicted PPI information [110] through interfacing different data bases such as IntAct Molecular Interaction Database (https://www.ebi.ac.uk/intact/) [111], Biological General Repository for Interaction Datasets (BioGRID) (https://thebiogrid.org/) [112], The Human Protein Reference Database (HPRD) (http://www.hprd.org/) [113], the Molecular INTeraction database (MINT) (https://mint.bio.uniroma2.it/) [114], The Biomolecular Interaction Network Database (BIND) (http://baderlab.org/BINDTranslation) [115], MIPS (http://mips.helmholtz-muenchen.de/proj/ppi/) [116] and DIP (http://dip.doembi.ucla.edu/dip/Main.cgi) [117]. In this study, the HIPPIE [110] was used to analyze the PPIs among the proteins encoded by the DEGs, then the PPI networks for the up-regulated and the down-regulated genes are separately visualized by Cytoscape version 3.5.1 software (http://www.cytoscape.org/) [118]. The degree of a gene in a PPI network is equal to the number of edges containing that node [119]. Betweenness centrality of a gene which is located on the shortest path between two other genes has most influence over the "information transfer" between them [120]. Stress centrality is number of genes in the shortest path between two other genes [121]. Closeness centrality is an inverse of the average length of the shortest paths to/from all the other genes in the PPI network [122]. Cluster coefficient measures the density of interactions in the network neighborhood of a gene [123].

\section{Module analysis}

In PPI networks, genes in the same module typically show the same or similar function and work together to implement their biological function. To visualize the network and identify the modules in the network, PEWCC1 java plug-in [124] on the Cytospace software (www.cytoscape.org/) [118] was used. The parameters were set as follows: Degree cutoff $\geq 10$ (degrees of each node in module were at 
least larger than 2), K-core $\geq 2$ (subgraphs of each node in module were at least 2 and more than 2).

\section{Construction of the target gene - miRNA network}

The NetworkAnalyst (http://www.networkanalyst.ca/) is a online tool available comprehensive resource containing the predicted and the experimentally validated target gene- miRNA interaction pairs [125]. The DEGs -associated predicted miRNA were selected when they were included two TarBase (http://diana.imis.athena-innovation.gr/DianaTools/index.php?r=tarbase/index) [126] and miRTarBase (http://mirtarbase.mbc.nctu.edu.tw/php/download.php) [127]. Subsequently, the overlapping target genes were identified and the genemiRNA pair was selected. The gene-miRNA network was formed and visualized using the Cytoscape version 3.5.1 software (http://www.cytoscape.org/) [118].

\section{Construction of the target gene - TF network}

The DEGs and transcription factors (TFs) that potentially regulated the DEGs are predicted using Overrepresentation Enrichment Analysis (ORA) in NetworkAnalyst (http://www.networkanalyst.ca/) [125]. The DEGs-associated predicted TF were selected when they were included database such as ENCODE (http://cistrome.org/BETA/) [128]. Then gene- TF- network are also visualized using version 3.5.1 software (http://www.cytoscape.org/) [118].

\section{Conclusion}

We use bioinformatics analysis of pituitary prolactinoma to investigate the biological and clinical value genes. Finally, using a series of particular conditions we screened crucial genes from DEGs. These findings may improve our understanding of the etiology, pathology, and the potential molecular mechanisms and gene targets of pituitary prolactinoma, which may be beneficial for the identification of diagnostic biomarkers and treatment methods for pituitary prolactinoma. Nevertheless, lacking of experimental verification is a limitation of this study. Further molecular biological experiments in vivo and in vitro are required to confirm the function of the identified genes in pituitary prolactinoma.

\section{Abbreviations}


BioGRID: Biological General Repository for Interaction Datasets; BP: biological process; CC: cellular component; DEGs : Differentially Expressed Genes; GEO : Gene Expression Omnibus; GO: Gene ontology; HIPPIE; Human Integrated Protein-Protein Interaction rEference; KEGG: Kyoto Encyclopedia of Genes and Genomes; MF: molecular function; SMPDB: Small Molecule Pathway Database

\section{Acknowledgement}

I thank $\mathrm{Ni} \mathrm{Li}$, Institute of Health Sciences, Shanghai Institute for Biological Sciences, Chinese Academy of Sciences, China, very much, the author who deposited their microarray dataset, GSE119063, into the public GEO database.

\section{Conflict of interest}

The authors declare that they have no conflict of interest.

\section{Ethical approval}

This article does not contain any studies with human participants or animals performed by any of the authors.

\section{Informed consent}

No informed consent because this study does not contain human or animals participants.

\section{Author Contributions}

Vikrant Ghatnatti - Methodology and validation

Basavaraj Vastrad - Writing original draft, investigation, and review and editing

Swetha Patil - Formal analysis and validation

Chanabasayya Vastrad - Software and investigation

Iranna Kotturshetti - Supervision and resources

\section{Availability of data and materials}


The datasets supporting the conclusions of this article are available in the GEO (Gene Expression Omnibus) (https://www.ncbi.nlm.nih.gov/geo/) repository. [(GSE119063)

(https://www.ncbi.nlm.nih.gov/geo/query/acc.cgi?acc=GSE119063)]

\section{Consent for publication}

Not applicable.

\section{Competing interests}

The authors declare that they have no competing interests.

\section{Authors}

Vikrant Ghatnatti

Basavaraj Vastrad

Swetha Patil

Chanabasayya Vastrad

Iranna Kotturshetti
ORCID ID: 0000-0001-9353-1481

ORCID ID: 0000-0003-2202-7637

ORCID ID: 0000-0003-2769-3970

ORCID ID: 0000-0003-3615-4450

ORCID ID: 0000-0003-1988-7345

\section{References}

1. Thorner, M.O.; Martin, W.H.; Rogol, A.D.; Morris, J.L.; Perryman, R.L.; Conway, B.P.; Howards, S.S.; Wolfman, M.G.; MacLeod, R.M. Rapid regression of pituitary prolactinomas during bromocriptine treatment. J Clin Endocrinol Metab. 1980,51,438-445. doi:10.1210/jcem-51-3-438

2. Doumith, R.; Gennes, J.L.; Cabane, J.P.; Zygelman, N. Pituitary prolactinoma, adrenal aldosterone-producing adenomas, gastric schwannoma and colonic polyadenomas: a possible variant of multiple endocrine neoplasia (MEN) type I. Acta Endocrinol (Copenh). 1982,100,189-195.

3. Oruçkaptan, H.H.; Senmevsim, O.; Ozcan, O.E.; Ozgen, T. Pituitary adenomas: results of 684 surgically treated patients and review of the literature. Surg Neurol. 2000,53,211-219. 
4. Cho, D.Y.; Liau, W.R. Comparison of endonasal endoscopic surgery and sublabial microsurgery for prolactinomas. Surg Neurol. 2002;58,371-375

5. Murakami, M.; Mizutani, A.; Asano, S.; Katakami, H.; Ozawa, Y.; Yamazaki, K.; Ishida, Y.; Takano, K.; Okinaga, H.; Matsuno, A. A mechanism of acquiring temozolomide resistance during transformation of atypical prolactinoma into prolactin-producing pituitary carcinoma: case report. Neurosurgery.

2011,68,E1761-E1767. doi:10.1227/NEU.0b013e318217161a

6. Tsang, R.W.; Laperriere, N.J.; Simpson, W.J.; Brierley, J.; Panzarella, T.; Smyth, H.S. Glioma arising after radiation therapy for pituitary adenoma. A report of four patients and estimation of risk. Cancer. 1993;72,2227-2233.

7. Friedman, E.; Adams, E.F.; Höög, A,; Gejman, P.V.; Carson, E.; Larsson, C.; De Marco, L.; Werner, S.; Fahlbusch, R.; Nordenskjöld, M. Normal structural dopamine type 2 receptor gene in prolactin-secreting and other pituitary tumors. $J$ Clin Endocrinol Metab. 1994,78,568-574. doi:10.1210/jcem.78.3.7907340

8. Fedele, M.; Pentimalli, F.; Baldassarre, G.; Battista, S.; Klein-Szanto, A.J.; Kenyon, L.; Visone, R.; De Martino, I.; Ciarmiello, A.; Arra, C.; et al. Transgenic mice overexpressing the wild-type form of the HMGA1 gene develop mixed growth hormone/prolactin cell pituitary adenomas and natural killer cell lymphomas. Oncogene. 2005,24,3427-3435. doi:10.1038/sj.onc.1208501

9. Finelli, P.; Pierantoni, G.M.; Giardino, D.; Losa, M.; Rodeschini, O.; Fedele, M.; Valtorta, E.; Mortini, P.; Croce, C.M.; Larizza, L.; et al. The High Mobility Group A2 gene is amplified and overexpressed in human prolactinomas. Cancer Res. 2002,62,2398-2405.

10.Paez-Pereda, M.; Giacomini, D.; Refojo, D.; Nagashima, A.C.; Hopfner, U.; Grubler, Y.; Chervin, A.; Goldberg, V.; Goya, R.; Hentges, S.T.; et al. Involvement of bone morphogenetic protein 4 (BMP-4) in pituitary prolactinoma pathogenesis through a Smad/estrogen receptor crosstalk. Proc Natl Acad Sci U S A. 2003,100,1034-1039. doi:10.1073/pnas.0237312100

11.Shimon, I.; Hinton, D.R.; Weiss, M.H.; Melmed, S. Prolactinomas express human heparin-binding secretory transforming gene (hst) protein product: marker of tumour invasiveness. Clin Endocrinol (Oxf). 1998;48(1):23-29. 
12.Lania, A.G.; Ferrero, S.; Pivonello, R.; Mantovani, G.; Peverelli, E.; Di Sarno, A.; Beck-Peccoz, P.; Spada, A.; Colao, A. Evolution of an aggressive prolactinoma into a growth hormone secreting pituitary tumor coincident with GNAS gene mutation. J Clin Endocrinol Metab. 2010,95,13-17. doi:10.1210/jc.2009-1360

13.Dworakowska, D.; Wlodek, E.; Leontiou, C.A.; Igreja, S.; Cakir, M.; Teng, M.; Prodromou, N. ;Góth, M.I.; Grozinsky-Glasberg, S.; Gueorguiev, M.; et al. Activation of RAF/MEK/ERK and PI3K/AKT/mTOR pathways in pituitary adenomas and their effects on downstream effectors. Endocr Relat Cancer. 2009,16,1329-1338. doi:10.1677/ERC-09-0101

14.Semba, S.; Han, S.Y.; Ikeda, H.; Horii, A. Frequent nuclear accumulation of beta-catenin in pituitary adenoma. Cancer. 2001,91,42-48.

15.Seemann, N.; Kuhn, D.; Wrocklage, C.; Keyvani, K.; Hackl, W.; Buchfelder, M.; Fahlbusch, R.; Paulus, W. CDKN2A/p16 inactivation is related to pituitary adenoma type and size. J Pathol. 2001,193,491-497. doi:10.1002/path.833

16.Ozfirat, Z.; Korbonits, M. AIP gene and familial isolated pituitary adenomas. Mol Cell Endocrinol. 2010,326,71-79. doi:10.1016/j.mce.2010.05.001

17.Lock, C.; Hermans, G.; Pedotti, R.; Brendolan, A.; Schadt, E.; Garren, H.; Langer-Gould, A.; Strober, S.; Cannella, B.; Allard, J.; et al. Genemicroarray analysis of multiple sclerosis lesions yields new targets validated in autoimmune encephalomyelitis. Nat Med. 2002,8,500-508. doi:10.1038/nm0502-500

18.Ma, C.; Wang, F.; Han, B.; Zhong, X.; Si, F.; Ye, J.; Hsueh, E.C.; Robbins, L.; Kiefer, S.M.; Zhang, Y.; et al. SALL1 functions as a tumor suppressor in breast cancer by regulating cancer cell senescence and metastasis through the NuRD complex. Mol Cancer. 2018,17,78. doi:10.1186/s12943-0180824-y

19.Wang, Y.C.; Gallego-Arteche, E.; Iezza, G.; Yuan, X.; Matli, M.R.; Choo, S.P.; Zuraek, M.B.; Gogia, R.; Lynn, F.C.; German, M.S.; et al. Homeodomain transcription factor NKX2.2 functions in immature cells to control enteroendocrine differentiation and is expressed in gastrointestinal neuroendocrine tumors. Endocr Relat Cancer. 2009,16,267-279. doi:10.1677/ERC-08-0127 
20.Alarmo, E.L. Pärssinen, J. Ketolainen, J.M. Savinainen, K. Karhu R. Kallioniemi, A. BMP7 influences proliferation, migration, and invasion of breast cancer cells. Cancer Lett. 2009,275,35-43. doi:10.1016/j.canlet.2008.09.028

21.Cipriano, R.; Miskimen, K.L.; Bryson, B.L.; Foy, C.R.; Bartel, C.A.; Jackson, M.W. Conserved oncogenic behavior of the FAM83 family regulates MAPK signaling in human cancer. Mol Cancer Res. 2014,12,11561165. doi:10.1158/1541-7786.MCR-13-0289

22.Quan, Y.; Xu, M.; Cui, P.; Ye, M.; Zhuang, B.; Min, Z. Grainyhead-like 2 Promotes Tumor Growth and is Associated with Poor Prognosis in Colorectal Cancer. J Cancer. 2015;6,342-350. doi:10.7150/jca.10969

23.Li, H. Sun, L. Tang, Z. Fu, L. Xu, Y. Li, Z. Luo, W. Qiu, X. Wang, E. Overexpression of TRIM24 correlates with tumor progression in non-small cell lung cancer. PLoS One. 2012,7,e37657. doi:10.1371/journal.pone.0037657

24.Kaistha, B.P.; Honstein, T.; Müller, V.; Bielak, S.; Sauer, M.; Kreider, R.; Fassan, M.; Scarpa, A.; Schmees, C.; Volkmer, H.; et al. Key role of dual specificity kinase TTK in proliferation and survival of pancreatic cancer cells. Br J Cancer. 2014;111,1780-1787. doi:10.1038/bjc.2014.460

25.Di Leo, A.; Desmedt, C.; Bartlett, J.M.; Piette, F.; Ejlertsen, B.; Pritchard, K.I.; Larsimont, D.; Poole, C.; Isola, J.; Earl, H.; et al. HER2 and TOP2A as predictive markers for anthracycline-containing chemotherapy regimens as adjuvant treatment of breast cancer: a meta-analysis of individual patient data. Lancet Oncol. 2011,12,1134-1142. doi:10.1016/S14702045(11)70231-5

26.Zhang, C.; Zhu, C; Chen, H.; Li, L.; Guo, L.; Jiang, W.; Lu, S.H. Kif18A is involved in human breast carcinogenesis. Carcinogenesis. 2010,31,16761684. doi:10.1093/carcin/bgq134

27.Aguirre-Portolés C, Bird AW, Hyman A, Cañamero M, Pérez de Castro I, Malumbres M. Tpx2 controls spindle integrity, genome stability, and tumor development. Cancer Res. 2012,72,1518-1528. doi:10.1158/00085472.CAN-11-1971

28.Zhu, X.; Lee, K.; Asa, S.L.; Ezzat, S. Epigenetic silencing through DNA and histone methylation of fibroblast growth factor receptor 2 in neoplastic 
pituitary cells. Am J Pathol. 2007,170,1618-1628. doi:10.2353/ajpath.2007.061111

29.Alatzoglou, K.S. Andoniadou, C.L. Kelberman, D. Buchanan, C.R. Crolla, J. Arriazu, M.C. Roubicek, M. Moncet, D. Martinez-Barbera, J.P. Dattani, M.T. SOX2 haploinsufficiency is associated with slow progressing hypothalamo-pituitary tumours. Hum Mutat. 2011,32,1376-1380. doi:10.1002/humu.21606

30.Heinrichs, M. Baumgärtner, W. Capen, C.C. Immunocytochemical demonstration of proopiomelanocortin-derived peptides in pituitary adenomas of the pars intermedia in horses. Vet Pathol. 1990,27,419-425. doi:10.1177/030098589902700606

31.Katznelson, L. Alexander, J.M. Bikkal, H.A. Jameson, J.L. Hsu, D.W. Klibanski, A. Imbalanced follicle-stimulating hormone beta-subunit hormone biosynthesis in human pituitary adenomas. J Clin Endocrinol Metab. 1992;74,1343-1351. doi:10.1210/jcem.74.6.1375599

32.Duong, C.V. Yacqub-Usman, K. Emes, R.D. Clayton, R.N. Farrell, W.E. The EFEMP1 gene: a frequent target for epigenetic silencing in multiple human pituitary adenoma subtypes. Neuroendocrinology. 2013;98,200-211. doi:10.1159/000355624

33.Wu, Y, Bai, J, Hong, L, Yu, S, Yu, G, Zhang, Y. Low expression of secreted frizzled-related protein 2 and nuclear accumulation of $\beta$-catenin in aggressive nonfunctioning pituitary adenoma. Oncol Lett. 2016;12,199-206. doi:10.3892/ol.2016.4560

34.Lekva, T.; Berg, J.P.; Lyle, R.; Heck, A.; Bollerslev, J.; Ueland, T. Alternative splicing of placental lactogen (CSH2) in somatotroph pituitary adenomas. Neuro Endocrinol Lett. 2015,36,136-142.

35.Yavropoulou, M.P.; Maladaki, A.; Topouridou, K.; Kotoula, V.; Poulios, C.; Daskalaki, E.; Foroglou, N.; Karkavelas, G.; Yovos, J.G. Expression pattern of the Hedgehog signaling pathway in pituitary adenomas. Neurosci Lett. 2016,611,94-100. doi:10.1016/j.neulet.2015.10.076

36.Giustina, A.; Bonfanti, C.; Licini, M.; De Rango, C.; Milani, G. Inhibitory effect of galanin on growth hormone release from rat pituitary tumor cells (GH1) in culture. Life Sci. 1994,55,1845-1851.

37.Hunter, J.A.; Skelly, R.H.; Aylwin, S.J.; Geddes, J.F.; Evanson, J.; Besser, G.M.; Monson, J.P.; Burrin, J.M. The relationship between pituitary tumour 
transforming gene (PTTG) expression and in vitro hormone and vascular endothelial growth factor (VEGF) secretion from human pituitary adenomas. Eur J Endocrinol. 2003,148,203-211.

38.De Martino, I.; Visone, R.; Wierinckx, A.; Palmieri, D.; Ferraro, A.; Cappabianca, P.; Chiappetta, G.; Forzati, F.; Lombardi, G.; Colao, A.; et al. HMGA proteins up-regulate CCNB2 gene in mouse and human pituitary adenomas. Cancer Res. 2009,69,1844-1850. doi:10.1158/0008-5472.CAN$08-4133$

39.Wierinckx, A.; Auger, C.; Devauchelle, P.; Reynaud, A.; Chevallier, P.; Jan, M.; Perrin, G.; Fèvre-Montange, M.; Rey, C.; Figarella-Branger, D.; et al. A diagnostic marker set for invasion, proliferation, and aggressiveness of prolactin pituitary tumors. Endocr Relat Cancer. 2007;14,887-900. doi:10.1677/ERC-07-0062

40.Tecimer, T. Dlott, J. Chuntharapai, A. Martin, A.W. Peiper, S.C. Expression of the chemokine receptor CXCR2 in normal and neoplastic neuroendocrine cells. Arch Pathol Lab Med. 2000;124,520-525.doi:10.1043/00039985(2000)124<0520:EOTCRC>2.0.CO;2

41.Grizzi, F. Borroni, E.M. Vacchini, A. Qehajaj, D. Liguori, M. Stifter, S. Chiriva-Internati, M. Di Ieva, A. Pituitary Adenoma and the Chemokine Network: A Systemic View. Front Endocrinol (Lausanne). 2015,6,141. doi:10.3389/fendo.2015.00141

42.Egashira, N.; Takekoshi, S.; Takei, M.; Teramoto, A.; Osamura, R.Y. Expression of FOXL2 in human normal pituitaries and pituitary adenomas. Mod Pathol. 2011,24,765-773. doi:10.1038/modpathol.2010.169

43.Zhang, H.Y. Jin, L. Stilling, G.A. Ruebel, K.H. Coonse, K. Tanizaki, Y. Raz, A. Lloyd, R.V. RUNX1 and RUNX2 upregulate Galectin-3 expression in human pituitary tumors. Endocrine. 2009,35,101-111. doi:10.1007/s12020-008-9129-Z

44.Tampanaru-Sarmesiu, A, Stefaneanu, L, Thapar, K, Kontogeorgos, G, Sumi, T, Kovacs, K. Transferrin and transferrin receptor in human hypophysis and pituitary adenomas. Am J Pathol. 1998; 152,413-422.

45.Rehfeld, J.F.; Lindholm, J.; Andersen, B.N.; Bardram, L.; Cantor, P.; Fenger, M.; Lüdecke, D.K. Pituitary tumors containing cholecystokinin. N Engl J Med. 1987;316,1244-1247. doi:10.1056/NEJM198705143162004 
46.Shaima, J.; William, B.; Omkaram, G.; et al. Developmental pluripotency associated 4: A novel putative predictor for prognosis of aggressive prolactin secreting tumors in the pituitary. Cancer Research. 2017,77.

47.Missale, C. Losa, M. Sigala, S. Balsari, A. Giovanelli, M. Spano, P.F. Nerve growth factor controls proliferation and progression of human prolactinoma cell lines through an autocrine mechanism. Mol Endocrinol. 1996,10,272285. doi:10.1210/mend.10.3.8833656

48.Tomlinson, D.C. Baldo, O. Harnden, P. Knowles, M.A. FGFR3 protein expression and its relationship to mutation status and prognostic variables in bladder cancer. J Pathol. 2007,213,91-98. doi:10.1002/path.2207

49.Shojima, K.; Sato, A.; Hanaki, H.; Tsujimoto, I.; Nakamura, M.; Hattori, K.; Sato, Y.; Dohi, K.; Hirata, M.; Yamamoto, H.; et al. Wnt5a promotes cancer cell invasion and proliferation by receptor-mediated endocytosis-dependent and -independent mechanisms, respectively. Sci Rep. 2015;5:8042. doi:10.1038/srep08042

50. Sharma P, Chinaranagari S, Patel D, et al. Epigenetic inactivation of inhibitor of differentiation 4 (Id4) correlates with prostate cancer. Cancer Med. 2012,1,176-186. doi:10.1002/cam4.16

51.Seder, C.W.; Hartojo, W.; Lin, L.; Carey, J.; Chaudhary, J. Upregulated INHBA expression may promote cell proliferation and is associated with poor survival in lung adenocarcinoma. Neoplasia. 2009;11(4):388-396.

52.Salem, C.E.; Markl, I.D.; Bender, C.M.; Gonzales, F.A.; Jones, P.A.; Liang, G. PAX6 methylation and ectopic expression in human tumor cells. Int $J$ Cancer. 2000,87,179-185.

53.Duan, J.J.; Cai, J.; Guo, Y.F.; Bian, X.W.; Yu, S.C.; ALDH1A3, a metabolic target for cancer diagnosis and therapy. Int J Cancer. 2016,139,965-975. doi:10.1002/ijc.30091

54.Xu, C.; Chen, H.; Wang, X.; Gao, J.; Che, Y.; Li, Y.; Ding, F.; Luo, A.; Zhang, S.; Liu, Z. S100A14, a member of the EF-hand calcium-binding proteins, is overexpressed in breast cancer and acts as a modulator of HER2 signaling. J Biol Chem. 2014,289,827-837. doi:10.1074/jbc.M113.469718

55.DeRycke, M.S.; Andersen, J.D.; Harrington, K.M.; Pambuccian, S.E.; Kalloger, S.E.; Boylan, K.L.; Argenta, P.A.; Skubitz, A.P.; S100A1 expression in ovarian and endometrial endometrioid carcinomas is a 
prognostic indicator of relapse-free survival. Am $J$ Clin Pathol. 2009,132,846-856. doi:10.1309/AJCPTK87EMMIKPFS

56.Harpio, R.; Einarsson, R. S100 proteins as cancer biomarkers with focus on $\mathrm{S} 100 \mathrm{~B}$ in malignant melanoma. Clin Biochem. 2004,37,512-528. doi:10.1016/j.clinbiochem.2004.05.012

57.Chen, H.; Suzuki, M.; Nakamura, Y.; Ohira, M.; Ando, S.; Iida, T.; Nakajima, T.; Nakagawara, A.; Kimura, H. Aberrant methylation of FBN2 in human non-small cell lung cancer. Lung Cancer. 2005,50,43-49. doi:10.1016/j.lungcan.2005.04.013

58.Yoshimura, N.; Sano, H.; Hashiramoto, A.; Yamada, R.; Nakajima, H.; Kondo, M.; Oka, T. The expression and localization of fibroblast growth factor-1 (FGF-1) and FGF receptor-1 (FGFR-1) in human breast cancer. Clin Immunol Immunopathol. 1998,89,28-34.

59.Dickinson, R.E. Dallol, A. Bieche, I. Krex, D. Morton, D. Maher, E.R. Latif, F. Epigenetic inactivation of SLIT3 and SLIT1 genes in human cancers. $\mathrm{Br} J$ Cancer. 2004,91,2071-2078. doi:10.1038/sj.bjc.6602222

60.Li, Z.; Zhang, W.; Shao, Y.; Zhang, C.; Wu, Q.; Yang, H.; Wan, X.; Zhang, J.; Guan, M.; Wan, J.; et al. High-resolution melting analysis of ADAMTS18 methylation levels in gastric, colorectal and pancreatic cancers. Med Oncol. 2010;27,998-1004. doi:10.1007/s12032-009-9323-8

61.Pei Y.F.; Zhang Y.J.; Lei Y.; Wu D.W.; Ma T.H.; Liu X.Q. Hypermethylation of the CHRDL1 promoter induces proliferation and metastasis by activating Akt and Erk in gastric cancer. Oncotarget. 2017,8,23155-23166. doi:10.18632/oncotarget.15513

62.Jacobs E.T.; Van Pelt C.; Forster R.E.; Zaidi W.; Hibler E.A.; Galligan M.A.; Haussler M.R.; Jurutka P.W. CYP24A1 and CYP27B1 polymorphisms modulate vitamin D metabolism in colon cancer cells. Cancer Res. 2013,73,2563-2573. doi:10.1158/0008-5472.CAN-12-4134

63.Liu, X.; Erikson, R. Polo-like kinase 1 in the life and death of cancer cells. Cell Cycle. 2003,2,424-425.

64.Grabsch, H, Takeno, S, Parsons, WJ, Pomjanski, N, Boecking, A, Gabbert, HE, Mueller, W. Overexpression of the mitotic checkpoint genes BUB1, BUBR1, and BUB3 in gastric cancer--association with tumour cell proliferation. J Pathol. 2003,200,16-22. doi:10.1002/path.1324 
65.Kitkumthorn, N.; Yanatatsanajit, P.; Kiatpongsan, S.; Phokaew, C.; Triratanachat, S.; Trivijitsilp, P.; Termrungruanglert, W.; Tresukosol, D.; Niruthisard, S.; Mutirangura, A. Cyclin A1 promoter hypermethylation in human papillomavirus-associated cervical cancer. BMC Cancer. 2006,6,55. doi:10.1186/1471-2407-6-55

66.Kato, T.; Wada, H.; Patel, P.; Hu, H.P.; Lee, D.; Ujiie, H.; Hirohashi, K.; Nakajima, T.; Sato, M.; Kaji, M.; et al. Overexpression of KIF23 predicts clinical outcome in primary lung cancer patients. Lung Cancer. 2016,92:5361. doi:10.1016/j.lungcan.2015.11.018

67.Cao, L.; Li, C.; Shen, S.; Yan, Y.; Ji, W.; Wang, J.; Qian, H.; Jiang, X.; Li, $\mathrm{Z}$; $\mathrm{Wu}, \mathrm{M}$; et al. OCT4 increases BIRC5 and CCND1 expression and promotes cancer progression in hepatocellular carcinoma. BMC Cancer. 2013,13,82. doi:10.1186/1471-2407-13-82

68.Zhang, Q.;Su, R.; Shan, C.; Gao, C.; Wu, P. Non-SMC Condensin I Complex, Subunit G (NCAPG) is a Novel Mitotic Gene Required for Hepatocellular Cancer Cell Proliferation and Migration. Oncol Res. 2018;26,269-276. doi:10.3727/096504017X15075967560980

69.Nie, W, Xu, MD, Gan, L, Huang, H, Xiu, Q, Li, B. Overexpression of stathmin 1 is a poor prognostic biomarker in non-small cell lung cancer. Lab Invest. 2015;95,56-64. doi:10.1038/labinvest.2014.124

70.Imai, K.; Hirata, S.; Irie, A.; Senju, S.; Ikuta, Y.; Yokomine, K.; Harao, M.; Inoue, M.; Tomita, Y.; Tsunoda, T.; et al. Identification of HLA-A2restricted CTL epitopes of a novel tumour-associated antigen, KIF20A, overexpressed in pancreatic cancer. $B r J$ Cancer. 2011,104,300-307. doi:10.1038/sj.bjc.6606052

71.Zhou, J.; Yu, Y.; Pei, Y.; Cao, C.; Ding, C.; Wang, D.; Sun, L.; Niu, G. A potential prognostic biomarker SPC24 promotes tumorigenesis and metastasis in lung cancer. Oncotarget. 2017,8,65469-65480. doi:10.18632/oncotarget.18971

72.He, W.L.; Li, Y.H.; Yang, D.J.; Song, W.; Chen, X.L.; Liu, F.K.; Wang, Z.; Li, W.; Chen, W.; Chen, C.Y.; et al. Combined evaluation of centromere protein $\mathrm{H}$ and $\mathrm{Ki}-67$ as prognostic biomarker for patients with gastric carcinoma. Eur J Surg Oncol. 2013,39,141-149. doi:10.1016/j.ejso.2012.08.023 
73.Hu, P.; Shangguan, J.; Zhang, L. Down regulation of NUF2 inhibits tumor growth and induces apoptosis by regulating lncRNA AF339813. Int J Clin Exp Pathol. 2015,8,2638-2648.

74.Pucci, F.; Rickelt, S,; Newton, A.P.; Garris, C.; Nunes, E.; Evavold, C.; Pfirschke, C.; Engblom, C.; Mino-Kenudson, M,; Hynes, R.O.; et al. PF4 Promotes Platelet Production and Lung Cancer Growth. Cell Rep. 2016;17,1764-1772. doi:10.1016/j.celrep.2016.10.031

75.Wilson, I.M.; Vucic, E.A.; Enfield, K.S.; Thu, K.L.; Zhang, Y.A.; Chari, R.; Lockwood, W.W.; Radulovich, N.; Starczynowski, D.T.; Banáth, J.P.; et al. EYA4 is inactivated biallelically at a high frequency in sporadic lung cancer and is associated with familial lung cancer risk. Oncogene. 2014,33,44644473. doi:10.1038/onc.2013.396

76.Ma, Y.; Qin, H.; Cui, Y. MiR-34a targets GAS1 to promote cell proliferation and inhibit apoptosis in papillary thyroid carcinoma via PI3K/Akt/Bad pathway. Biochem Biophys Res Commun. 2013;441,958-963. doi:10.1016/j.bbrc.2013.11.010

77.Xu, Y.; Zhang, X.; Hu, X.; Zhou, W.; Zhang, P.; Zhang, J.; Yang, S.; Liu, Y. The effects of lncRNA MALAT1 on proliferation, invasion and migration in colorectal cancer through regulating SOX9. Mol Med. 2018;24,52. doi:10.1186/s10020-018-0050-5

78.Mei, H.; Lian, S.; Zhang, S.; Wang, W.; Mao, Q.; Wang, H. High expression of ROR2 in cancer cell correlates with unfavorable prognosis in colorectal cancer. Biochem Biophys Res Commun. 2014,453,703-709. doi:10.1016/j.bbrc.2014.09.141

79.Gordon, C.A.; Gulzar, Z.G.; Brooks, J.D. NUSAP1 expression is upregulated by loss of RB1 in prostate cancer cells. Prostate. 2015,75,517526. doi:10.1002/pros.22938

80.Kang, M.A.; Kim, J.T.; Kim, J.H.; Kim, S.Y.; Kim, Y.H.; Yeom, Y.I.; Lee, Y.; Lee, H.G. Upregulation of the cycline kinase subunit CKS2 increases cell proliferation rate in gastric cancer. $J$ Cancer Res Clin Oncol. 2009;135,761-769. doi:10.1007/s00432-008-0510-3

81.Park, J.H.; Lin, M.L.; Nishidate, T.; Nakamura, Y.; Katagiri, T. PDZbinding kinase/T-LAK cell-originated protein kinase, a putative cancer/testis antigen with an oncogenic activity in breast cancer. Cancer Res. 2006,66,9186-9195. doi:10.1158/0008-5472.CAN-06-1601 
82.Hayward, D.G.; Clarke, R.B.; Faragher, A.J.; Pillai, M.R.; Hagan, I.M.; Fry, A.M. The centrosomal kinase Nek2 displays elevated levels of protein expression in human breast cancer. Cancer Res. 2004,64,7370-7376. doi:10.1158/0008-5472.CAN-04-0960

83.Corson, T.W.; Huang, A.; Tsao, M.S.; Gallie, B.L. KIF14 is a candidate oncogene in the $1 \mathrm{q}$ minimal region of genomic gain in multiple cancers. Oncogene. 2005,24,4741-4753. doi:10.1038/sj.onc.1208641

84.Wong, A,K.; Pero, R.; Ormonde, P.A.; Tavtigian, S.V.; Bartel, P.L. RAD51 interacts with the evolutionarily conserved BRC motifs in the human breast cancer susceptibility gene brca2. J Biol Chem. 1997,272,31941-31944.

85.Loveday, C.; Turnbull, C.; Ramsay, E.; Hughes, D.; Ruark, E.; Frankum, J.R.; Bowden, G.; Kalmyrzaev, B.; Warren-Perry, M.; Snape, K.; et al. Germline mutations in RAD51D confer susceptibility to ovarian cancer. Nat Genet. 2011,43,879-882. doi:10.1038/ng.893

86.Hasegawa, S.; Eguchi, H.; Nagano, H.; Konno, M.; Tomimaru, Y.; Wada, H.; Hama, N.; Kawamoto, K.; Kobayashi, S.; Nishida, N.; et al. MicroRNA1246 expression associated with CCNG2-mediated chemoresistance and stemness in pancreatic cancer. $B r \quad J$ Cancer. 2014,111,1572-1580. doi:10.1038/bjc.2014.454

87.Paramasivam M, Sarkeshik A, Yates JR et al Angiomotin family proteins are novel activators of the LATS2 kinase tumor suppressor. Mol Biol Cell. 2011;22(19):3725-3733. doi:10.1091/mbc.E11-04-0300

88.Kosaka, Y.; Inoue, H.; Ohmachi, T.; Fernandes, M.J.; McCollum, D. Tripartite motif-containing 29 (TRIM29) is a novel marker for lymph node metastasis in gastric cancer. Ann Surg Oncol. 2007;14,2543-2549. doi:10.1245/s10434-007-9461-1

89.Shang, B.; Gao, A.; Pan, Y.; Zhang, G.; Tu, J.; Zhou, Y.; Yang, P.; Cao, Z.; Wei, Q.; Ding, Y.; et al. CT45A1 acts as a new proto-oncogene to trigger tumorigenesis and cancer metastasis. Cell Death Dis. 2014,5,e1285. doi:10.1038/cddis.2014.244

90.Jing, Y.; Nguyen, M.M.; Wang, D.; Pascal, L.E.; Guo ,W.; Xu, Y.; Ai, J.; Deng, F.M.; Masoodi, K.Z.; Yu, X.; et al. DHX15 promotes prostate cancer progression by stimulating Siah2-mediated ubiquitination of androgen receptor. Oncogene. 2018;37,638-650. doi:10.1038/onc.2017.371 
91.Subhash, V.V.; Tan, S.H.; Tan, W.L.; Yeo, M.S.; Xie, C.; Wong, F.Y.; Kiat, Z.Y.; Lim, R.; Yong, W.P. GTSE1 expression represses apoptotic signaling and confers cisplatin resistance in gastric cancer cells. BMC Cancer. 2015,15,550. doi:10.1186/s12885-015-1550-0

92.Alvarez-Fernández, M.; Medema, R.H. Novel functions of FoxM1: from molecular mechanisms to cancer therapy. Front Oncol. 2013,3,30. doi:10.3389/fonc.2013.00030

93.Scharer, C.D.; McCabe, C.D.; Ali-Seyed, M.; Berger, M.F.; Bulyk, M.L.; Moreno, C.S. Genome-wide promoter analysis of the SOX4 transcriptional network in prostate cancer cells. Cancer Res. 2009;69,709-717. doi:10.1158/0008-5472.CAN-08-3415

94.Hu, J.Y.; Yi, W.; Wei, X.; Zhang, M.Y.; Xu, R.; Zeng, L.S.; Huang, Z.J.; Chen, J.S. miR-601 is a prognostic marker and suppresses cell growth and invasion by targeting PTP4A1 in breast cancer. Biomed Pharmacother. 2016,79,247-253. doi:10.1016/j.biopha.2016.02.014

95.Wanajo, A.; Sasaki, A.; Nagasaki, H.; Shimada, S.; Otsubo, T.; Owaki, S.; Shimizu, Y.; Eishi, Y.; Kojima, K.; Nakajima, Y.; et al. Methylation of the calcium channel-related gene, CACNA2D3, is frequent and a poor prognostic factor in gastric cancer. Gastroenterology. 2008,135,580-590. doi:10.1053/j.gastro.2008.05.041

96.Jia, Y.; Yang, Y.; Brock, M.V.; Zhan, Q.; Herman, J.G.; Guo, M. Epigenetic regulation of DACT2, a key component of the Wnt signalling pathway in human lung cancer. J Pathol. 2013;230,194-204. doi:10.1002/path.4073

97.Diboun, I.; Wernisch, L.; Orengo, C.A.; Koltzenburg, M. Microarray analysis after RNA amplification can detect pronounced differences in gene expression using limma. BMC Genomics. 2006,7,252. doi:10.1186/14712164-7-252

98. Reiner-Benaim, A. FDR control by the BH procedure for two-sided correlated tests with implications to gene expression data analysis. Biom J. 2007,49,107-126.

99.Caspi, R.; Billington, R.; Ferrer, L.; Foerster, H.; Fulcher, C.A.; Keseler, I.M.; Kothari, A.; Krummenacker, M.; Latendresse, M.; Mueller, L.A.; et al. The MetaCyc database of metabolic pathways and enzymes and the BioCyc collection of pathway/genome databases. Nucleic Acids Res. 2016,44,D471D480. doi:10.1093/nar/gkv1164 
100. Kanehisa, M.; Sato, Y.; Furumichi, M.; Morishima, K.; Tanabe, M. New approach for understanding genome variations in KEGG. Nucleic Acids Res. 2019,47,D590-D595. doi:10.1093/nar/gky962

101. Schaefer, C.F.; Anthony, K.; Krupa, S.; Buchoff, J.; Day, M.; Hannay, T.; Buetow, K.H. PID: the Pathway Interaction Database. Nucleic Acids Res 2009,37,D674-D679. doi:10.1093/nar/gkn653

102. Fabregat, A.; Jupe, S.; Matthews, L.; Sidiropoulos, K.; Gillespie, M.; Garapati, P.; Haw, R.; Jassal, B.; Korninger, F.; May, B.; et al. The Reactome Pathway Knowledgebase. Nucleic Acids Res. 2018,46,D649D655. doi:10.1093/nar/gkx1132

103. Dahlquist, K.D.; Salomonis, N.; Vranizan, K.; Lawlor, S.C.; Conklin, B.R. GenMAPP, a new tool for viewing and analyzing microarray data on biological pathways. Nat Genet. 2002;31,19-20. doi:10.1038/ng0502-19

104. Subramanian, A, Tamayo, P, Mootha, VK, Mukherjee, S.; Ebert, B.L.; Gillette, M.A.; Paulovich, A.; Pomeroy, S.L.; Golub, T.R.; Lander, E.S.; et al. Gene set enrichment analysis: a knowledge-based approach for interpreting genome-wide expression profiles. Proc Natl Acad Sci. U. S. A. 2005,102,15545-15550. doi:10.1073/pnas.0506580102

105. Mi, H.; Huang, X.; Muruganujan, A.; Tang, H.; Mills, C.; Kang, D.;

Thomas, P.D. PANTHER version 11: expanded annotation data from Gene Ontology and Reactome pathways, and data analysis tool enhancements. Nucleic Acids Res. 2017;45,D183-D189. doi:10.1093/nar/gkw1138

106. Petri, V.; Jayaraman, P.; Tutaj, M.; Hayman, G.T.; Smith, J.R.; De

Pons, J.; Laulederkind, S.J.; Lowry, T.F.; Nigam, R.; Wang, S.J.; et al. The pathway ontology - updates and applications. J Biomed Semantics. 2014,5,7. doi:10.1186/2041-1480-5-7

107. Jewison, T.; Su, Y.; Disfany, F.M.; Liang, Y.; Knox, C.; Maciejewski, A.; Poelzer, J.; Huynh, J.; Zhou, Y.; Arndt, D.; et al. SMPDB 2.0: big improvements to the Small Molecule Pathway Database. Nucleic Acids Res.

2014,42,D478-D484. doi:10.1093/nar/gkt1067

108. Chen, J.;Bardes, E.E.; Aronow, B.J.; Jegga, A.G. ToppGene Suite for gene list enrichment analysis and candidate gene prioritization. Nucleic Acids Res. 2009,37,W305-W311. doi:10.1093/nar/gkp427

109. Ashburner, M.; Ball, C.A.; Blake, J.A.; Botstein, D.;Butler, H.; Cherry, J.M.; Davis, A.P.; Dolinski, K.; Dwight, S.S.; Eppig, J.T.; et al. 
Gene ontology: tool for the unification of biology. The Gene Ontology Consortium. Nat Genet. 2000,25,25-29. doi:10.1038/75556

110. Alanis-Lobato, G.; Andrade-Navarro, M.A.; Schaefer, M.H. HIPPIE v2.0: enhancing meaningfulness and reliability of protein-protein interaction networks. Nucleic Acids Res. 2017,45,D408-D414. doi:10.1093/nar/gkw985

111. Orchard, S.; Ammari, M.; Aranda, B.; Breuza, L.; Briganti, L.; Broackes-Carter, F.; Campbell, N.H.; Chavali, G.; Chen, C.; del-Toro, N.; et al. The MIntAct project--IntAct as a common curation platform for 11 molecular interaction databases. Nucleic Acids Res. 2014;42,D358-D363. doi:10.1093/nar/gkt1115

112. Chatr-Aryamontri, A, Oughtred, R, Boucher, L, Rust, J, Chang, C, Kolas, NK, O'Donnell, L, Oster, S, Theesfeld, C, Sellam, A et al. The BioGRID interaction database: 2017 update. Nucleic Acids Res. 2017,45,D369-D379. doi:10.1093/nar/gkw1102

113. Keshava Prasad, TS, Goel, R, Kandasamy, K, Keerthikumar, S,

Kumar, S, Mathivanan, S, Telikicherla, D, Raju, R, Shafreen, B, Venugopal, A et al. Human Protein Reference Database--2009 update. Nucleic Acids Res. 2009,37,D767-D772. doi:10.1093/nar/gkn892

114. Licata, L.; Briganti, L.; Peluso, D.; Perfetto, L.; Iannuccelli, M.; Galeota, E.; Sacco, F.; Palma, A.; Nardozza, A.P.; Santonico, E.; et al. MINT, the molecular interaction database: 2012 update. Nucleic Acids Res. 2012;40,D857-D861. doi:10.1093/nar/gkr930

115. Isserlin, R, El-Badrawi, RA, Bader, GD. The Biomolecular Interaction Network Database in PSI-MI 2.5. Database (Oxford). 2011,2011,baq037. doi:10.1093/database/baq037

116. Pagel, P.; Kovac, S.; Oesterheld, M.; Dunger-Kaltenbach, I.; Frishman, G.; Montrone, C.; Mark, P.; Stümpflen, V.; Mewes, H.W.; Ruepp, A.; et al. The MIPS mammalian protein-protein interaction database. Bioinformatics. 2005;21,832-834. doi:10.1093/bioinformatics/bti115

117. Salwinski, L, Miller, CS, Smith, AJ, Pettit, FK, Bowie, JU, Eisenberg, D. The Database of Interacting Proteins: 2004 update. Nucleic Acids Res. 2004,32,D449-D451. doi:10.1093/nar/gkh086

118. Shannon, P, Markiel, A, Ozier, O, Baliga, NS, Wang, JT, Ramage, D, Amin, N, Schwikowski, B, Ideker, T. Cytoscape: a software environment for 
integrated models of biomolecular interaction networks. Genome Res. 2003,13,2498-2504. doi:10.1101/gr.1239303

119. Przulj, N, Wigle, DA, Jurisica, I. Functional topology in a network of protein interactions. Bioinformatics. $\quad \mathbf{2 0 0 4 , 2 0 , 3 4 0 - 3 4 8 . ~}$ doi:10.1093/bioinformatics/btg415

120. Joy, M.P.; Brock, A.; Ingber, D.E.; Huang, S. High-betweenness proteins in the yeast protein interaction network. J Biomed Biotechnol. 2005,2005,96-103. doi:10.1155/JBB.2005.96

121. Lehtinen, S, Marsellach, FX, Codlin, S, Schmidt, A, Clément-Ziza, M, Beyer, A, Bähler, J, Orengo, C, Pancaldi, V. Stress induces remodelling of yeast interaction and co-expression networks. Mol Biosyst. 2013,9,16971707. doi:10.1039/c3mb25548d

122. Hsu, C.W.; Juan, H.F.; Huang, H.C. Characterization of microRNAregulated protein-protein interaction network. Proteomics. 2008,8,19751979. doi:10.1002/pmic.200701004

123. Stelzl, U.; Worm, U,; Lalowski, M,; Haenig, C.; Brembeck, F.H.; Goehler, H.; Stroedicke, M.; Zenkner, M.; Schoenherr, A.; Koeppen, S.; et al. A human protein-protein interaction network: a resource for annotating the proteome. Cell. 2005,122,957-968. doi:10.1016/j.cell.2005.08.029

124. Zaki, N, Efimov, D, Berengueres, J. Protein complex detection using interaction reliability assessment and weighted clustering coefficient. BMC Bioinformatics. 2013,14,163. doi:10.1186/1471-2105-14-163

125. Xia, J.; Benner, M.J.; Hancock, R.E. NetworkAnalyst--integrative approaches for protein-protein interaction network analysis and visual exploration. Nucleic Acids Res. 2014,42,W167-w174. doi:10.1093/nar/gku443

126. Vlachos, I.S.; Paraskevopoulou, M.D.; Karagkouni, D.; Georgakilas, G.; Vergoulis, T.; Kanellos, I.; Anastasopoulos, I.L.; Maniou, S.; Karathanou, K.; Kalfakakou, D.; et al. DIANA-TarBase v7.0: indexing more than half a million experimentally supported miRNA:mRNA interactions. Nucleic Acids Res. 2015,43,D153-D159. doi:10.1093/nar/gku1215

127. Chou, C.H.; Shrestha, S.; Yang, C.D.; Chang, N.W.; Lin, Y.L.; Liao, K.W.; Huang, W.C.; Sun, T.H.; Tu, S.J.; Lee, W.H.; et al. miRTarBase update 2018: a resource for experimentally validated microRNA-target 
interactions. Nucleic Acids Res. 2018,46,D296-D302. doi:10.1093/nar/gkx1067

128. Wang, S.; Sun, H.; Ma, J.; Zang, C.; Wang, C.; Wang, J.; Tang, Q.; Meyer, C.A.; Zhang, Y.; Liu, X.S. Target analysis by integration of transcriptome and ChIP-seq data with BETA. Nat Protoc. 2013,8,25022515. doi:10.1038/nprot.2013.150
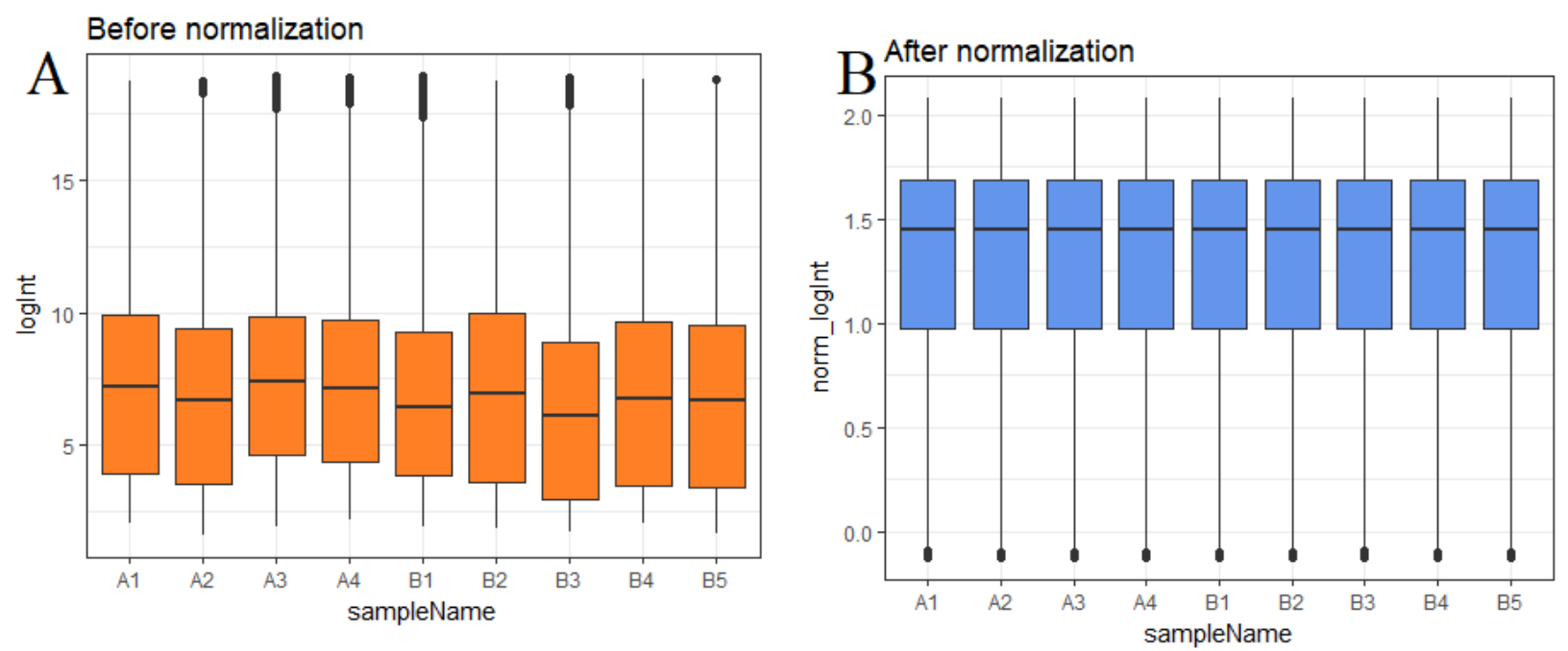

Fig. 1. Box plots of the gene expression data before and after normalization. Horizontal axis represents the sample symbol and the vertical axis represents the gene expression values. The black line in the box plot represents the median value of gene expression. (A1, A2, A3, A4 = normal pituitaries samples; B1, B2, B3, B4, B5 $=5$ pituitary prolactinoma ) 

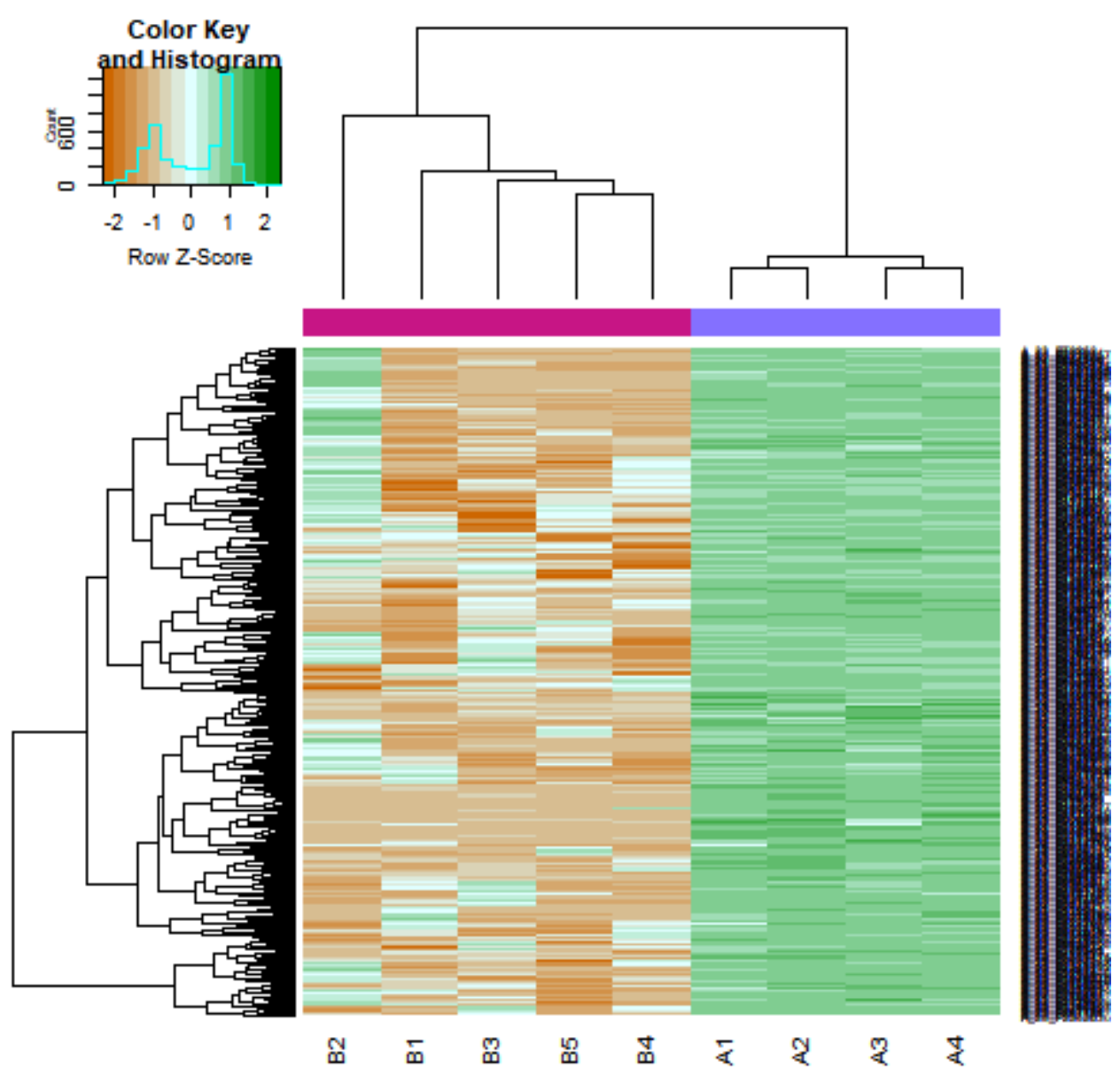

Fig. 2. Heat map of up regulated differentially expressed genes. Legend on the top left indicate $\log$ fold change of genes. (A1, A2, A3, A4 = normal pituitaries samples; B1, B2, B3, B4, B5 = 5 pituitary prolactinoma ) 


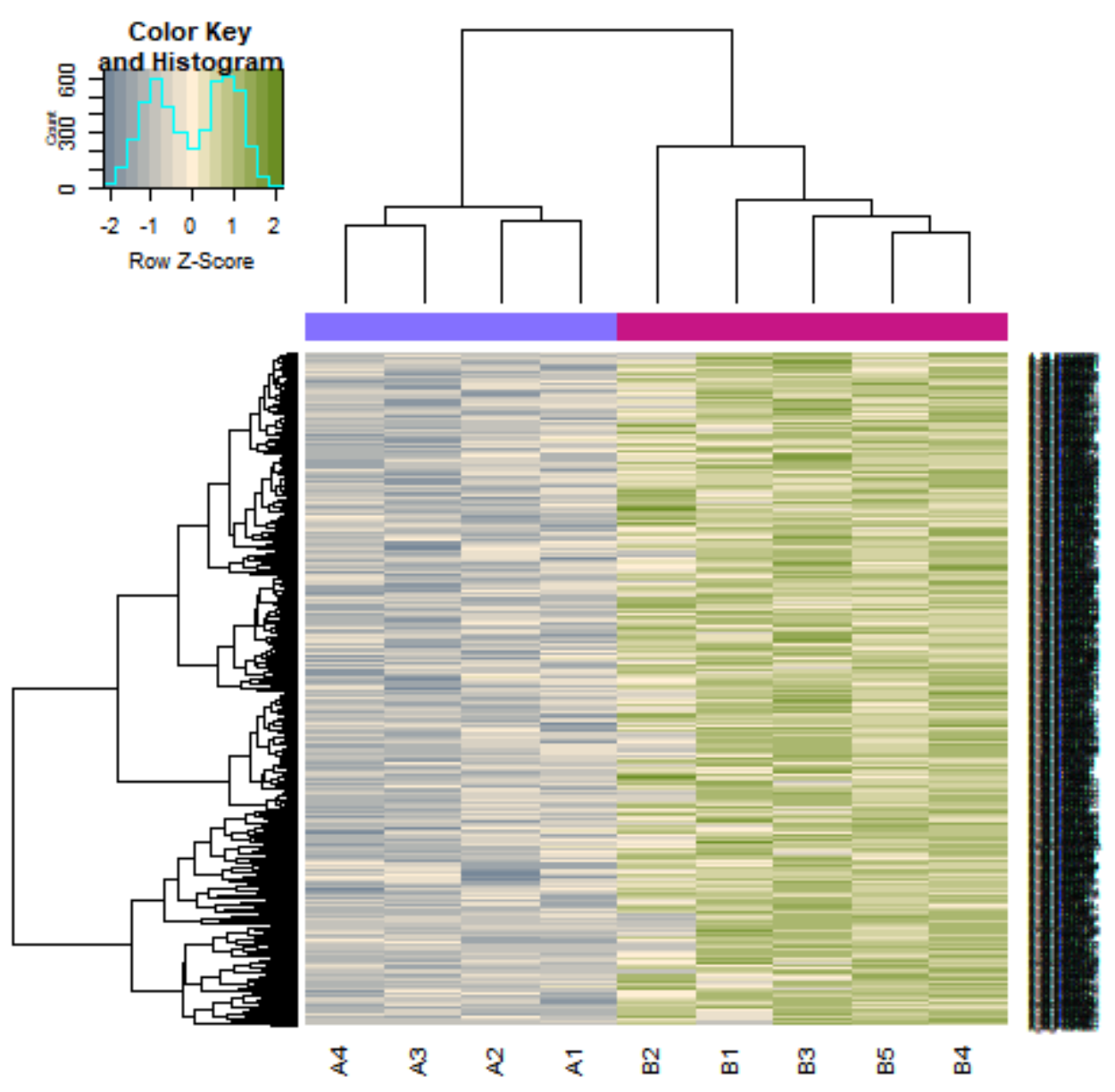

Fig. 3. Heat map of down regulated differentially expressed genes. Legend on the top left indicate $\log$ fold change of genes. (A1, A2, A3, A4 = normal pituitaries samples; B1, B2, B3, $\mathrm{B} 4, \mathrm{~B} 5=5$ pituitary prolactinoma ) 


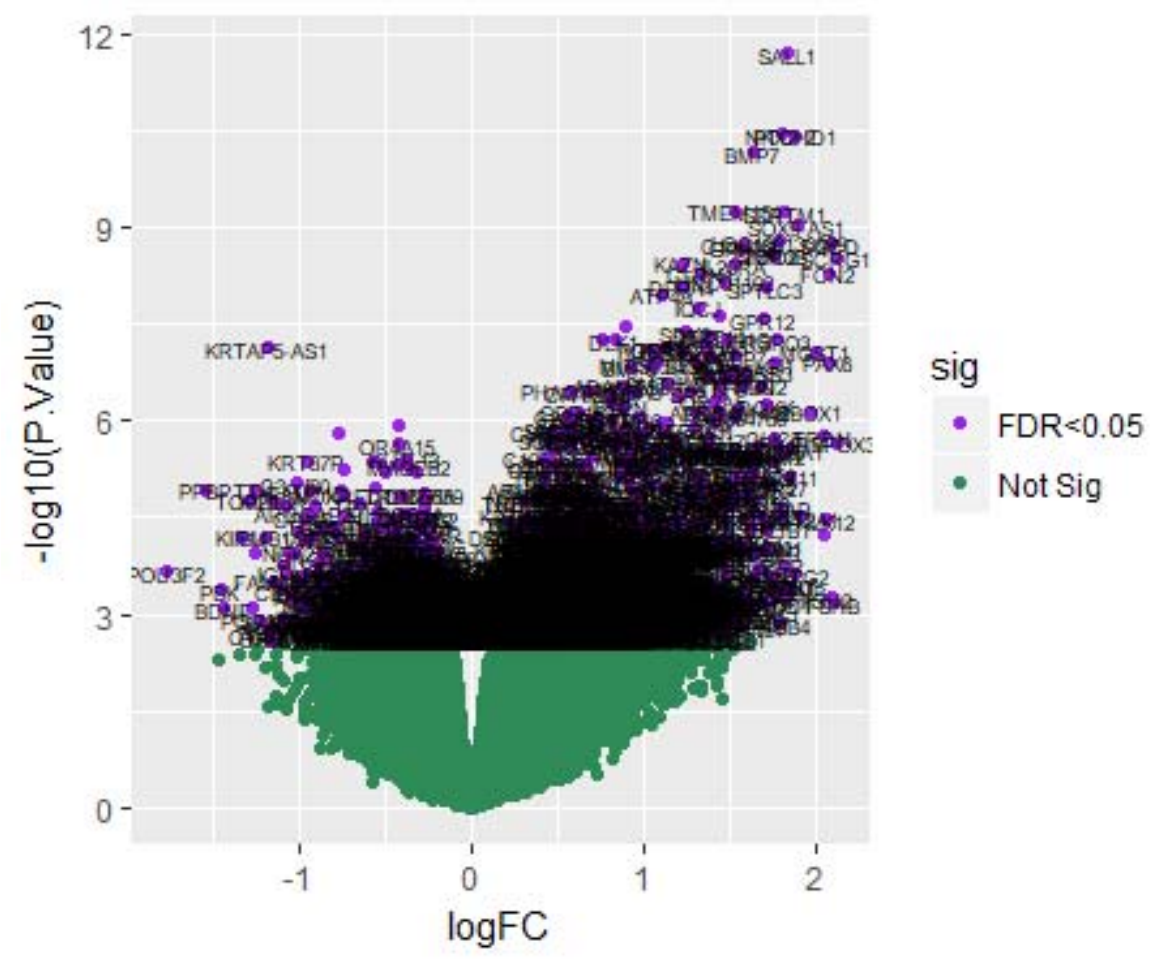

Fig. 4. Volcano plot of differentially expressed genes. Genes with a significant change of more than two-fold were selected. 


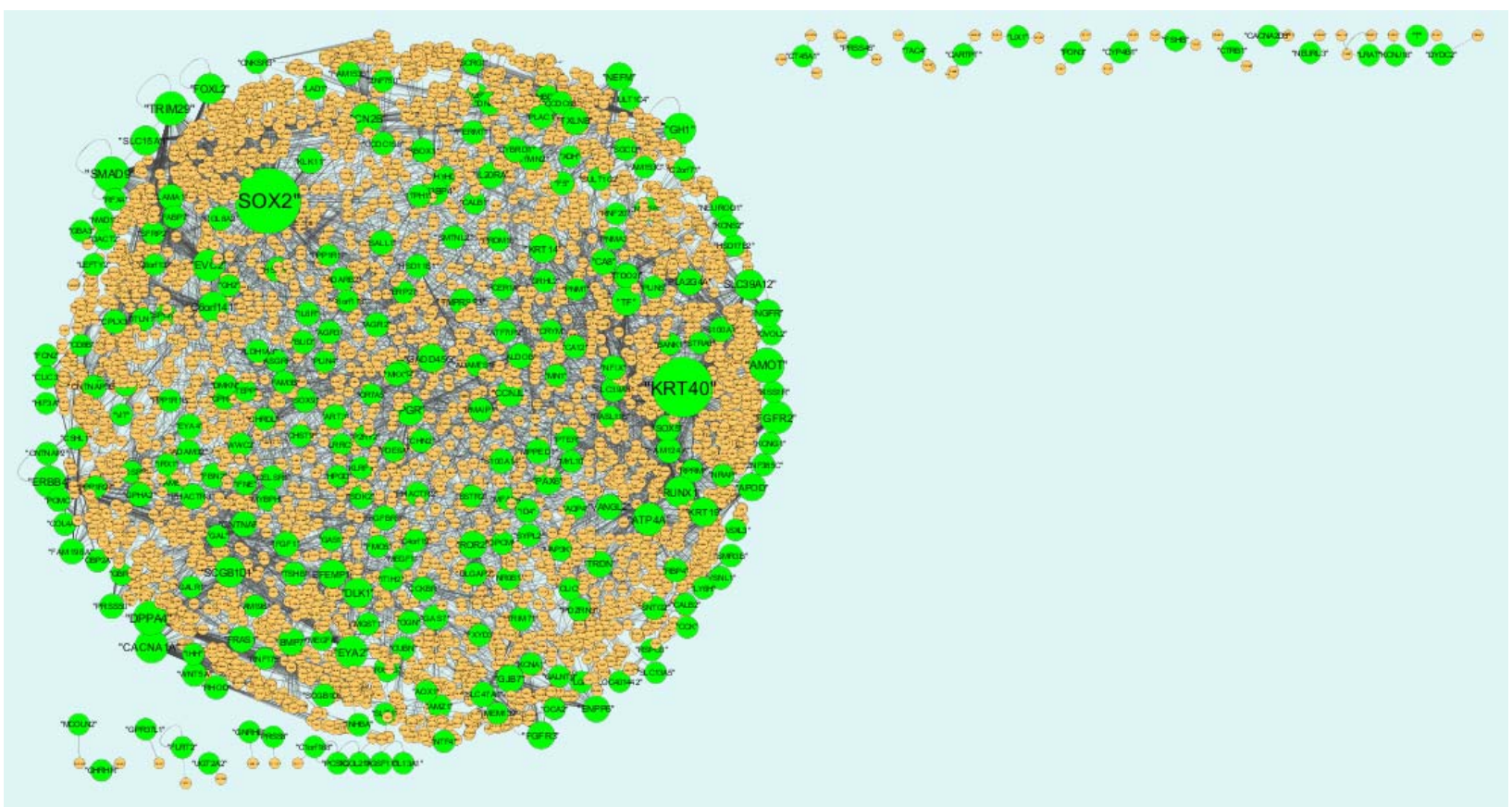

Fig. 5. Protein-protein interaction network of differentially expressed genes (DEGs). Green nodes denotes up regulated genes.

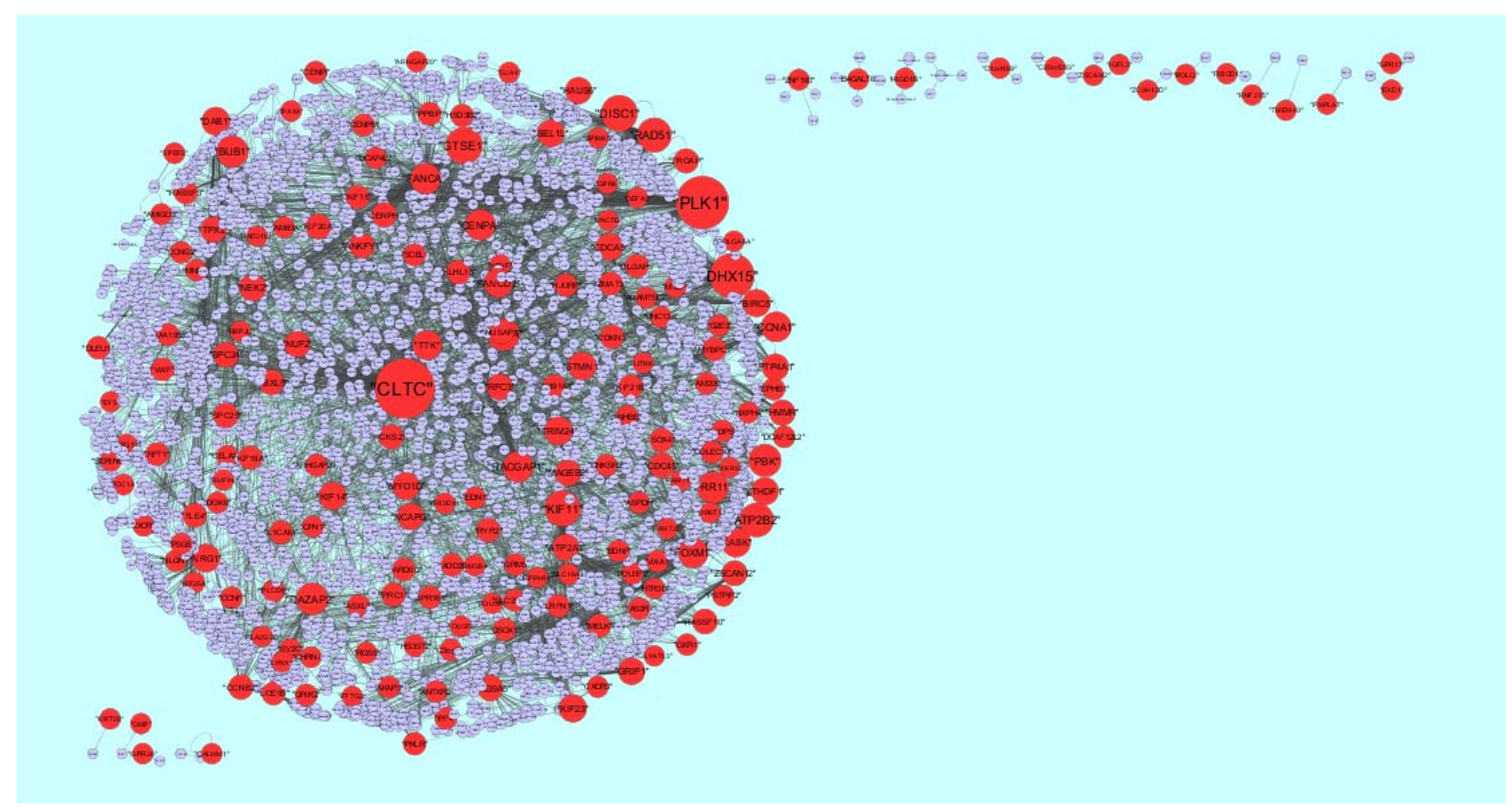

Fig. 6. Protein-protein interaction network of differentially expressed genes (DEGs). Red nodes denotes up regulated genes. 

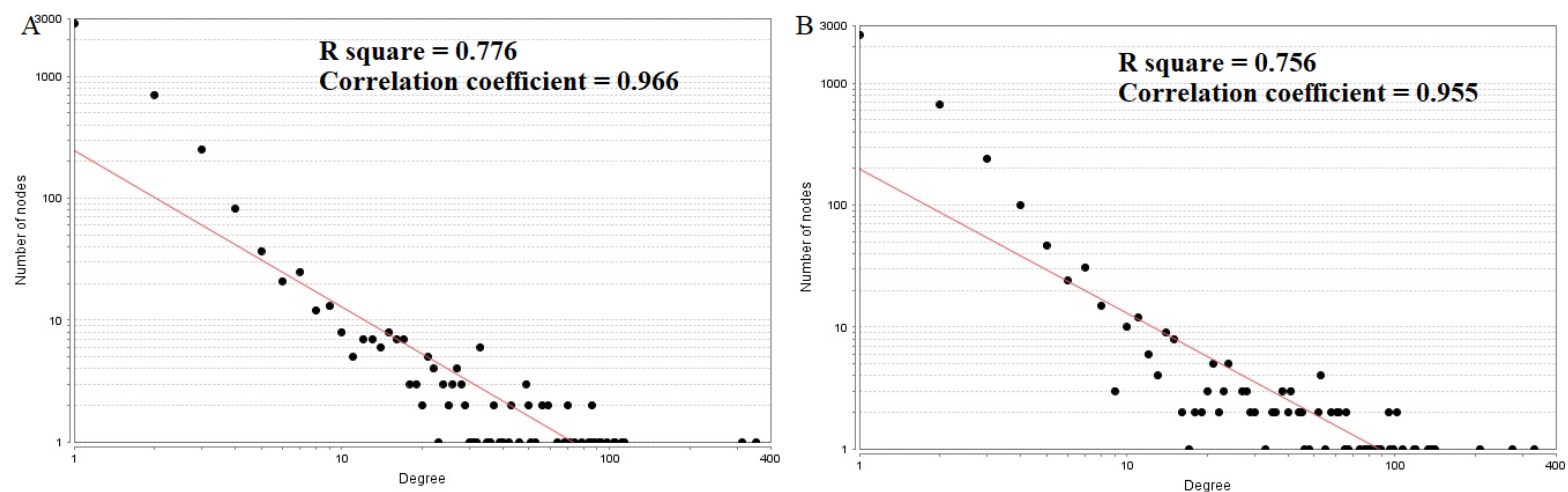

Fig. 7. Node degree distribution (A- Up regulated genes ; B- - Down regulated genes)
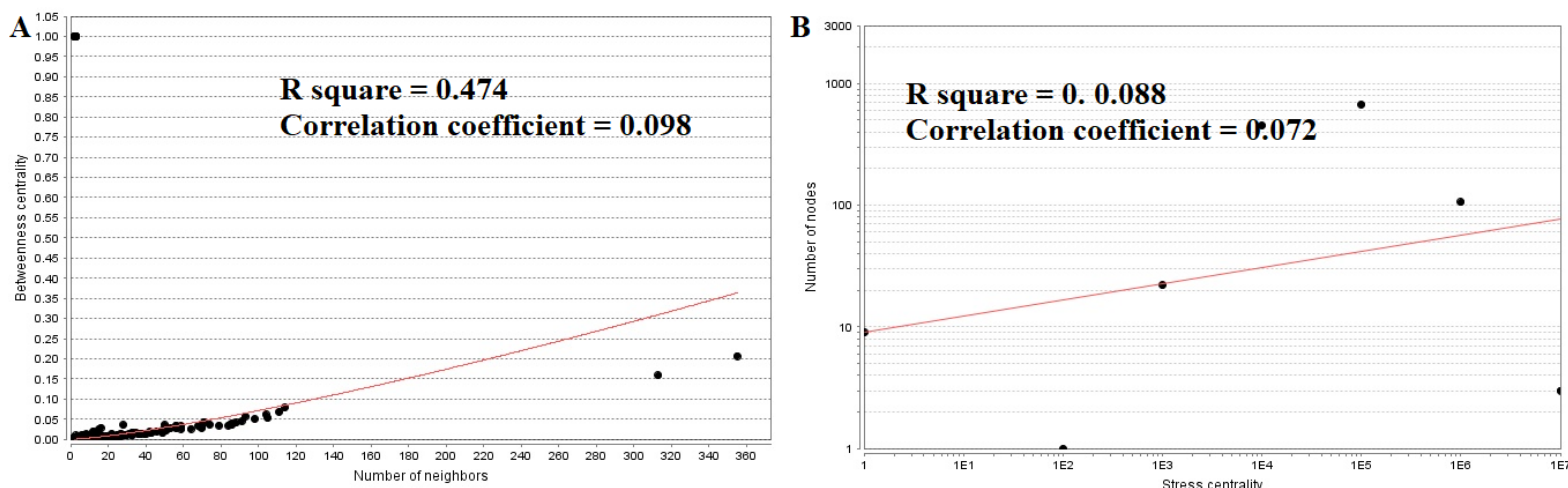

C

D
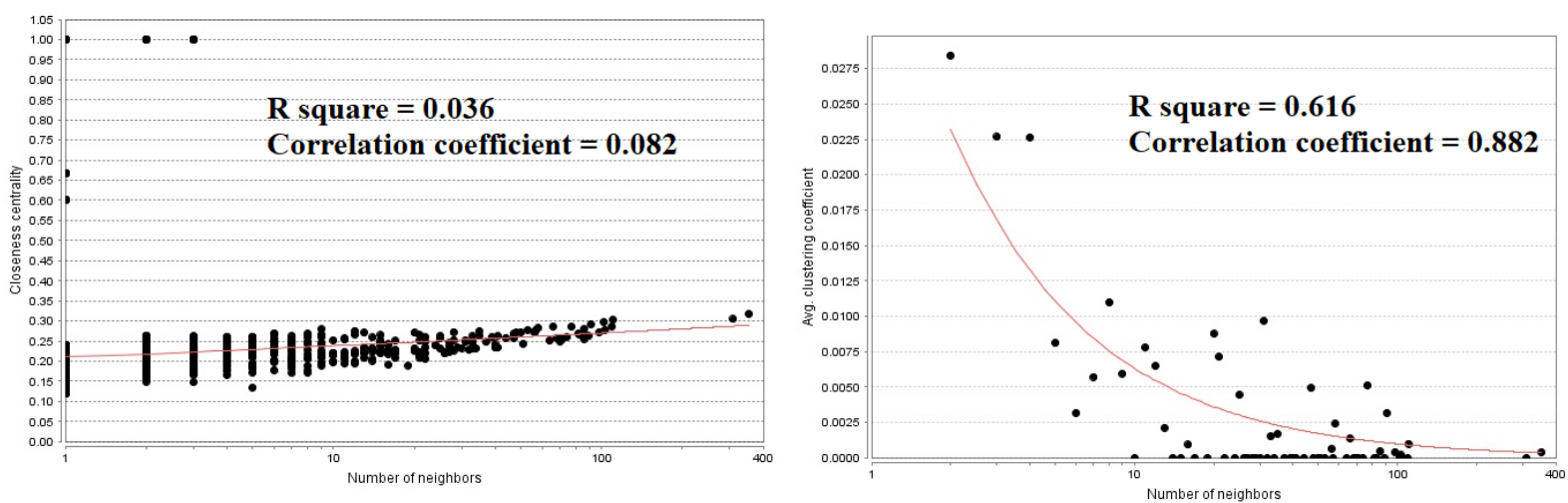

Fig. 8. Regression diagrams for Up regulated genes ( A- Betweenness centrality; B- Stress centrality ; C- Closeness centrality; D- Clustering coefficient) 

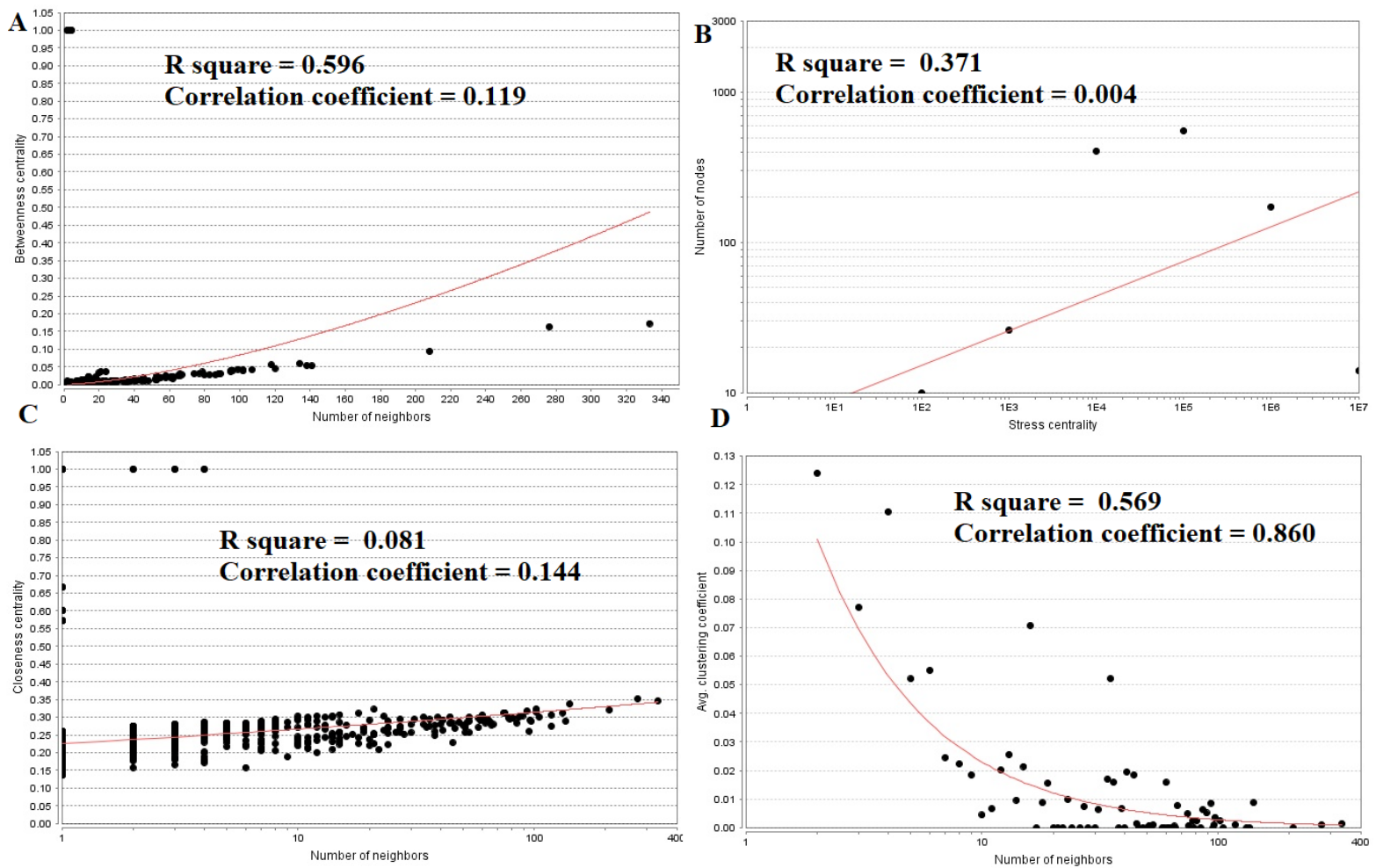

Fig. 9. Regression diagrams for Down regulated genes ( A- Betweenness centrality; B- Stress centrality ; C-Closeness centrality; D- Clustering coefficient) 
Module 1

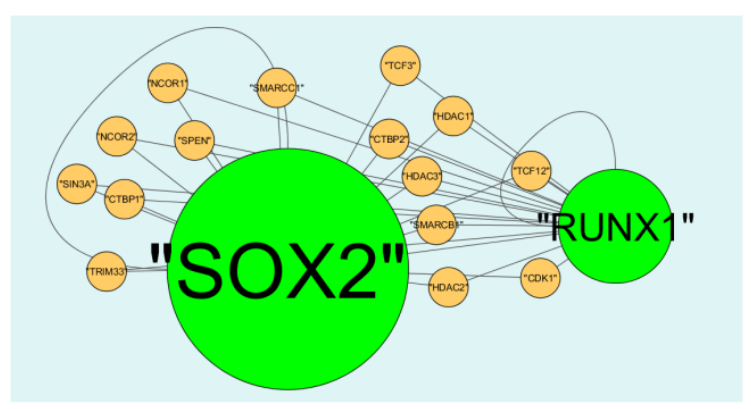

Module 3

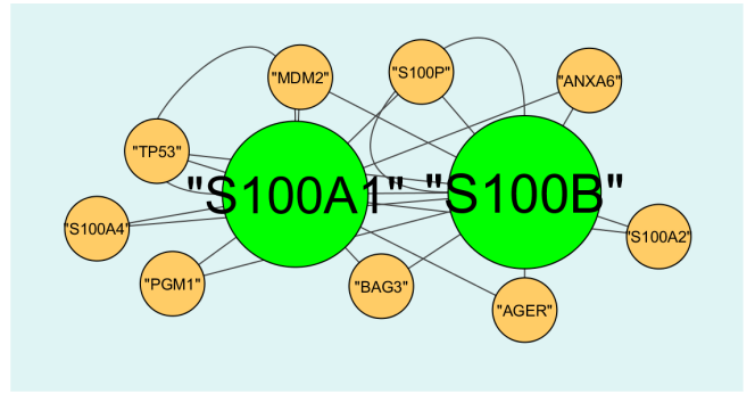

Module 2

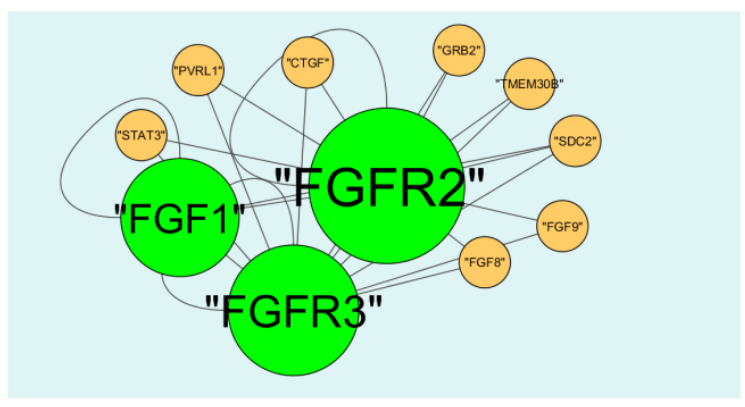

Module 10

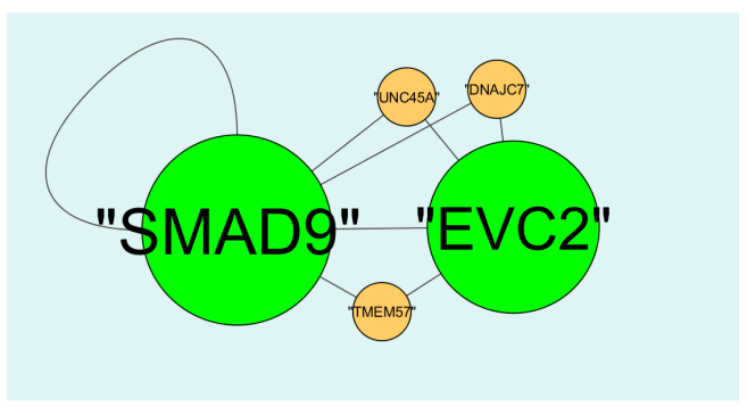

Fig. 10. Modules in PPI network. The green nodes denote the up-regulated genes. 


\section{Module 1}

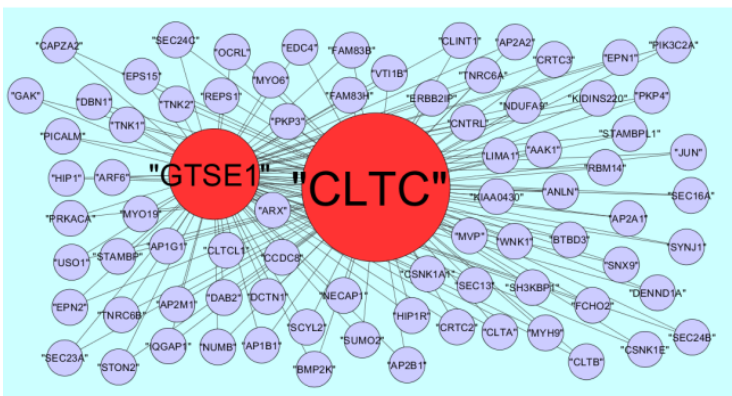

Module 8

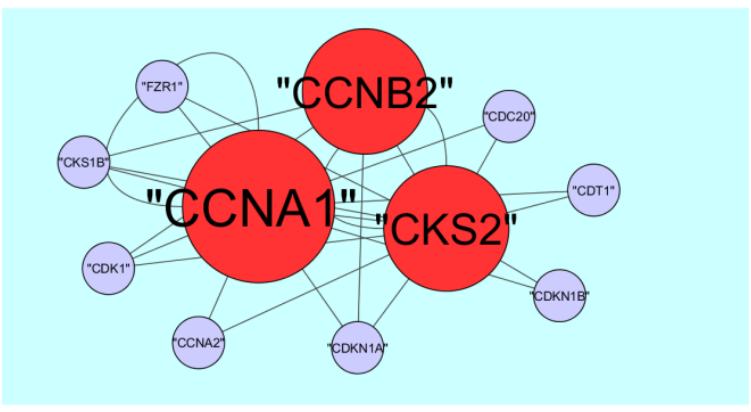

Module 5

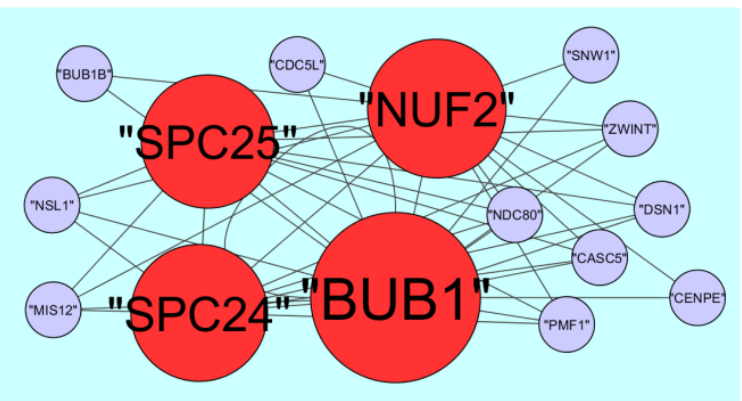

Module 12

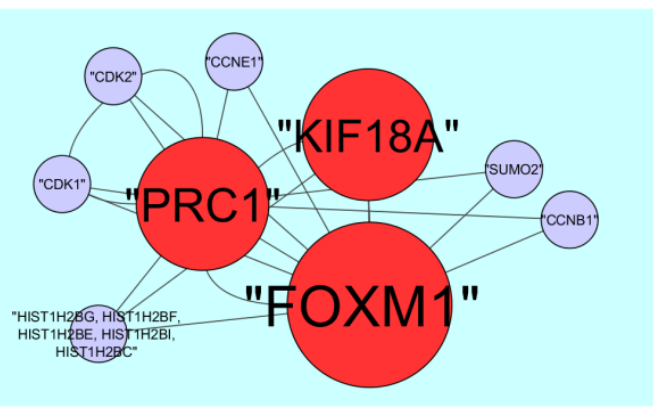

Fig. 11. Modules in PPI network. The red nodes denote the down-regulated genes. 


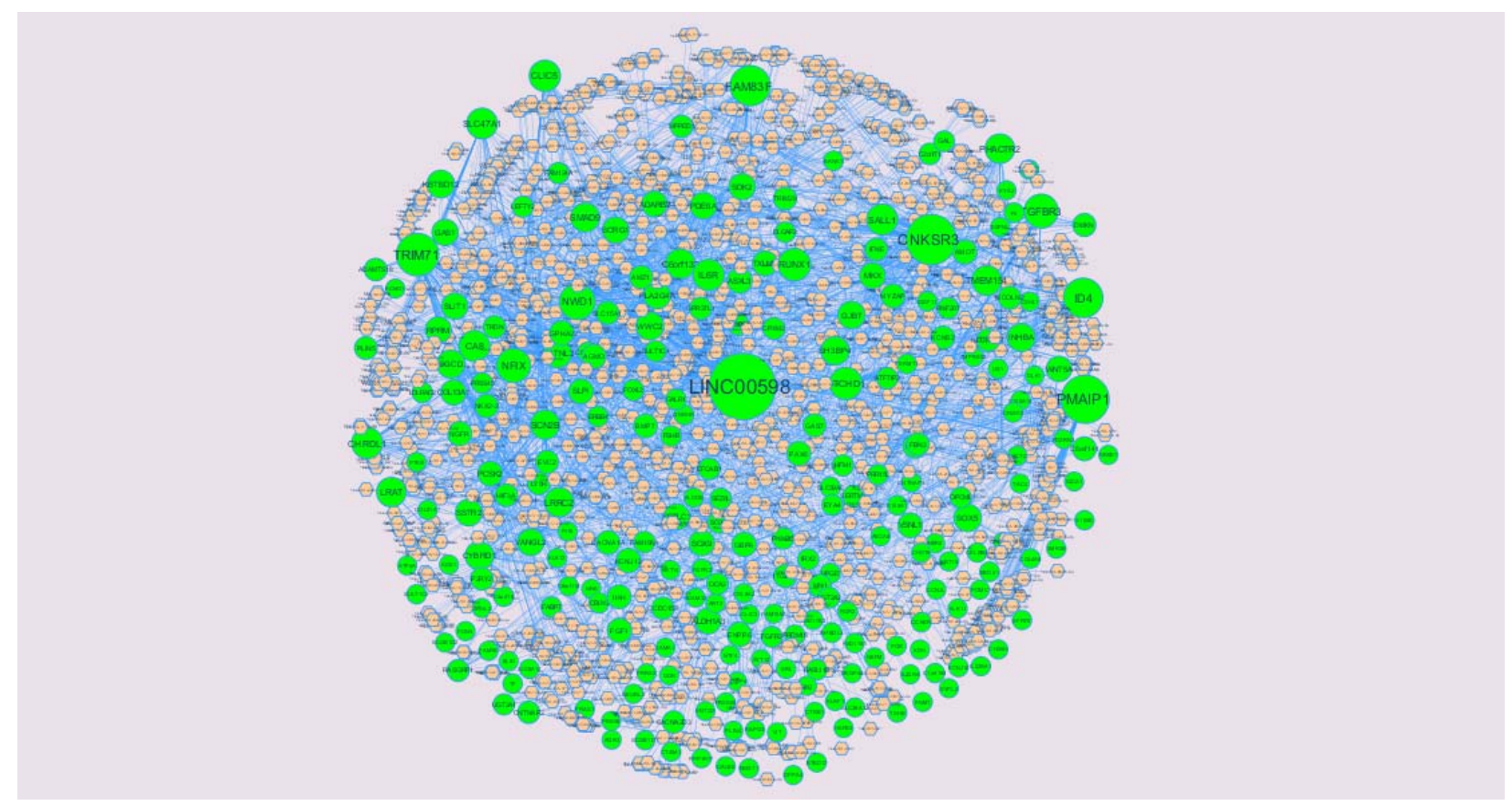

Fig. 12. The network of up-regulated DEGs and their related miRNAs. The green circles nodes are the up regulated DEGs and pinkiamond nodes are the miRNAs 


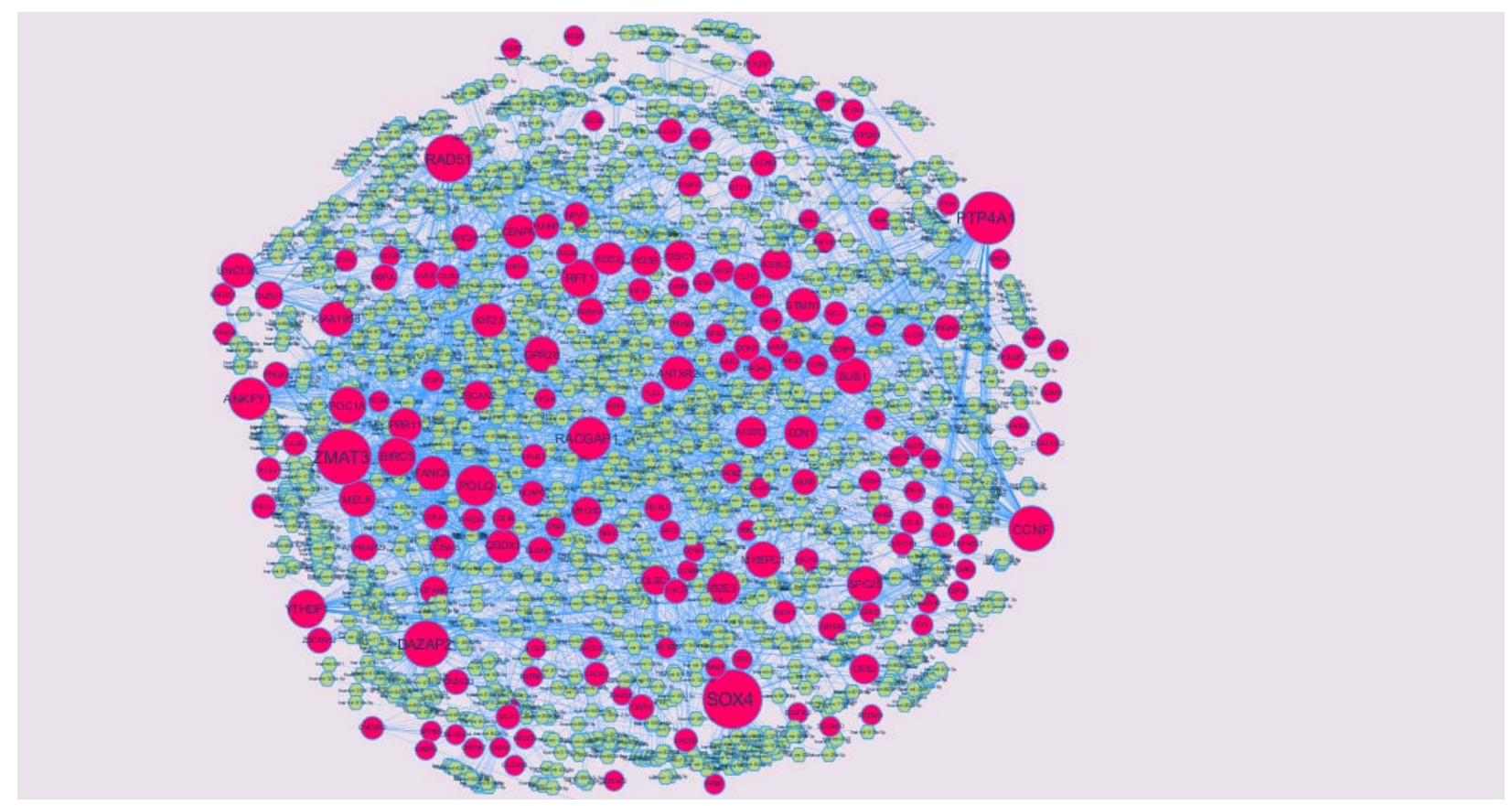

Fig. 13. The network of up-regulated DEGs and their related miRNAs. The pink circles nodes are the up regulated DEGs and yellow diamond nodes are the miRNAs 


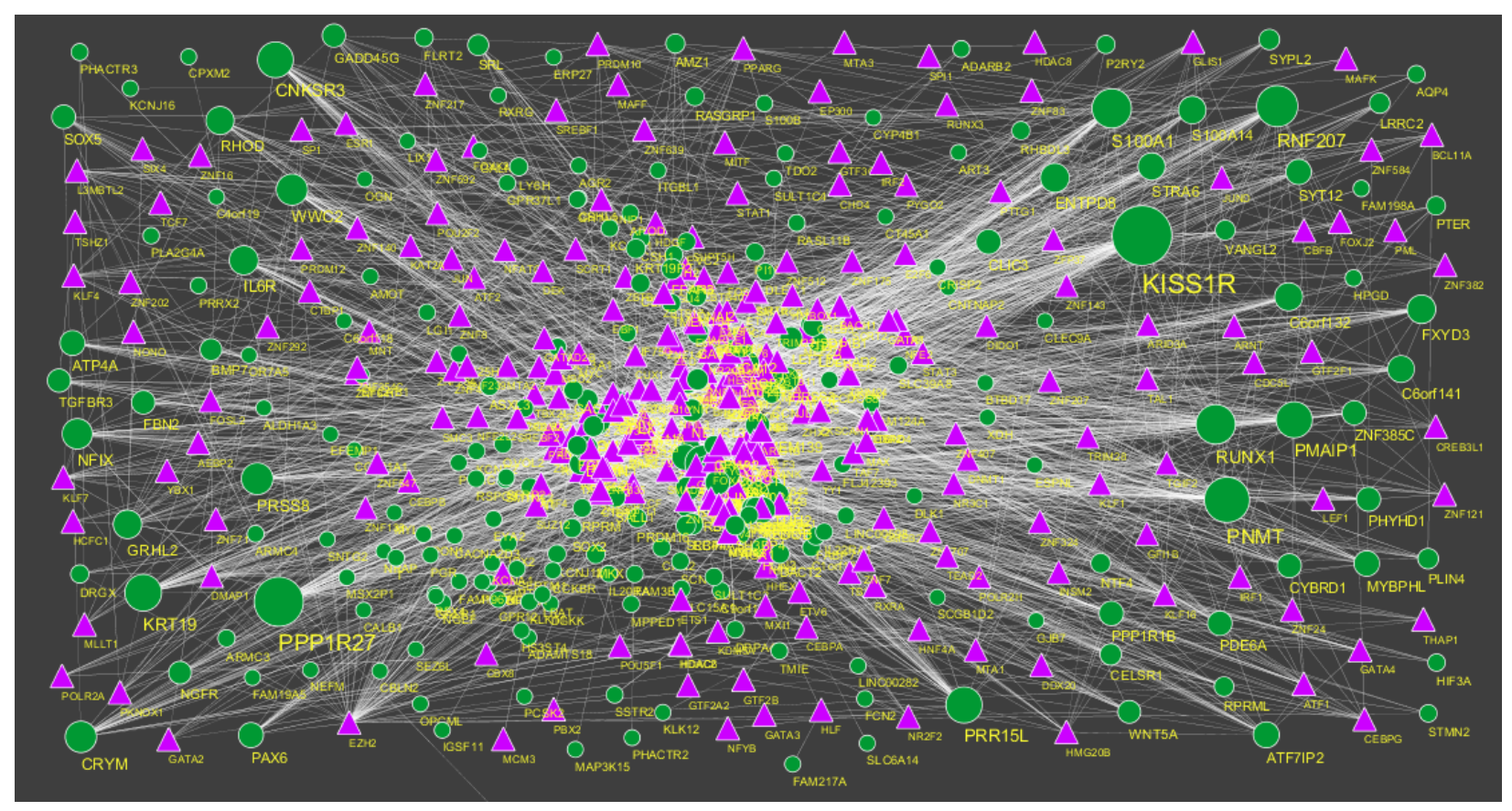

Fig. 14. TF - gene network of predicted target up regulated genes. (Purple triangle - TFs and green circles- target up regulated genes) 


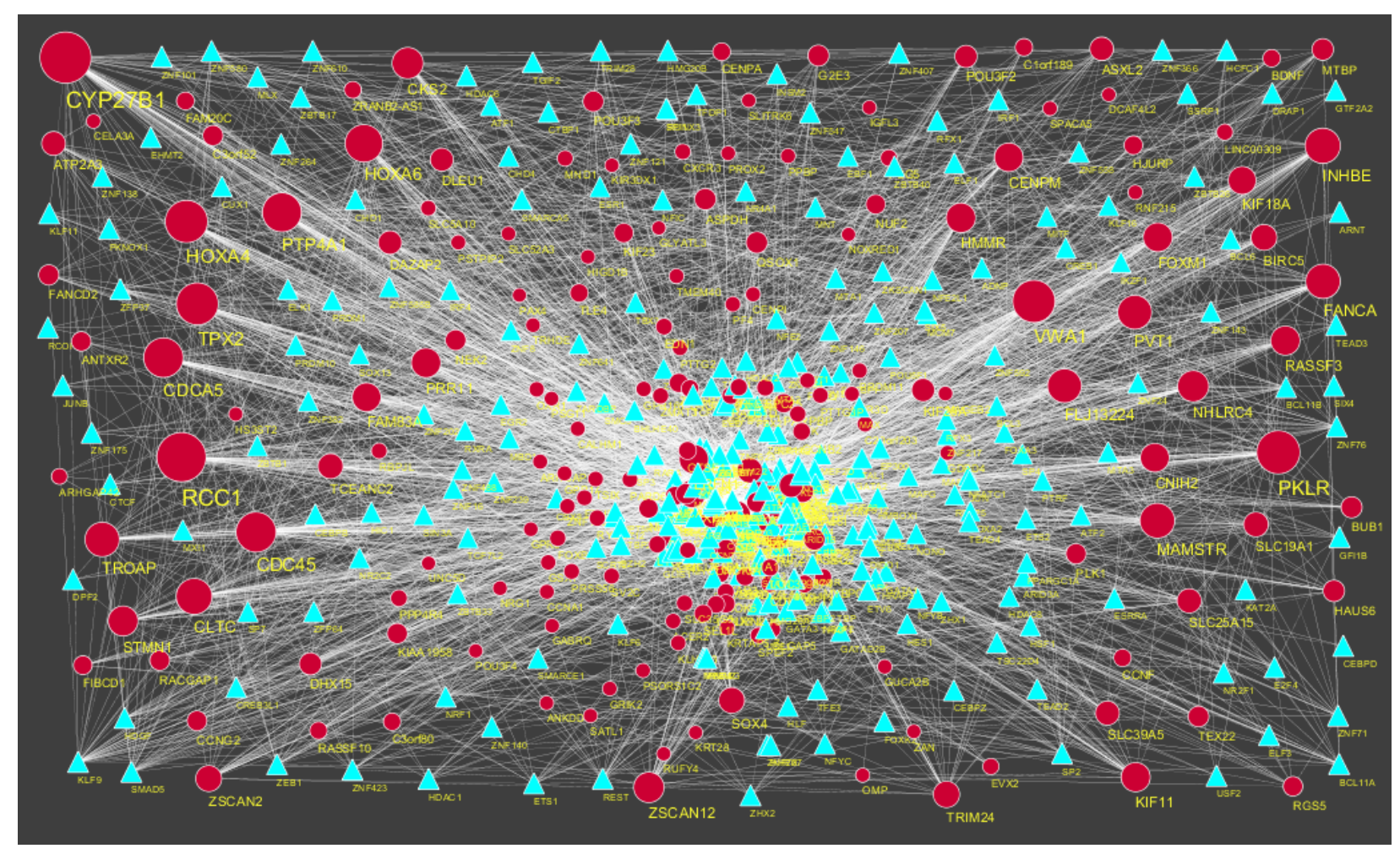

Fig. 15. TF- gene network of predicted target up regulated genes. (Sky blue triangle - TFs and red circles- target up regulated genes)

\section{Tables}

Table 1 The statistical metrics for key differentially expressed genes (DEGs)

\begin{tabular}{|c|c|c|c|c|c|c|c|}
\hline Agilent Id & Gene Symbol & $\log$ FC & P Value & FDR & T value & Up/Down & Gene Name \\
\hline A_23_P328074 & SALL1 & 1.833089 & $2.06 \mathrm{E}-12$ & 8.77E-08 & 60.26576 & Up & spalt like transcription factor 1 \\
\hline A_23_P17522 & NKX2-2 & 1.814078 & $3.68 \mathrm{E}-11$ & $5.54 \mathrm{E}-07$ & 42.7718 & Up & NK2 homeobox 2 \\
\hline A_32_P149435 & PTCHD1 & 1.891448 & $3.91 \mathrm{E}-11$ & $5.54 \mathrm{E}-07$ & 42.46841 & Up & patched domain containing 1 \\
\hline A_23_P68487 & BMP7 & 1.638544 & 7.17E-11 & 7.63E-07 & 39.50907 & Up & bone morphogenetic protein 7 \\
\hline A_23_P361584 & TMEM154 & 1.53654 & $5.79 \mathrm{E}-10$ & 4.13E-06 & 30.7916 & Up & $\begin{array}{l}\text { transmembrane protein } 154 \\
\text { serine rich and transmembrane domain containing }\end{array}$ \\
\hline A_24_P366607 & SERTM1 & 1.817216 & $5.83 \mathrm{E}-10$ & 4.13E-06 & 30.77075 & $\mathrm{Up}$ & 1 \\
\hline A_24_P497464 & SOX9-AS1 & 1.905462 & $9.38 \mathrm{E}-10$ & $5.7 \mathrm{E}-06$ & 29.06852 & Up & SOX9 antisense RNA 1 \\
\hline A_33_P3240747 & FAM83F & 1.788916 & $1.62 \mathrm{E}-09$ & $8.1 \mathrm{E}-06$ & 27.23397 & Up & family with sequence similarity 83 member $F$ \\
\hline A_32_P4595 & SGCD & 2.094348 & $1.81 \mathrm{E}-09$ & $8.1 \mathrm{E}-06$ & 26.87211 & $\mathrm{Up}$ & sarcoglycan delta \\
\hline A_32_P182299 & C1orf168 & 1.588213 & 1.93E-09 & $8.1 \mathrm{E}-06$ & 26.65684 & Up & chromosome 1 open reading frame 168 \\
\hline A_24_P35228 & GRHL2 & 1.574365 & 2.1E-09 & $8.1 \mathrm{E}-06$ & 26.40034 & Up & grainyhead like transcription factor 2 \\
\hline A_23_P80974 & TDO2 & 1.745047 & $2.66 \mathrm{E}-09$ & $9.04 \mathrm{E}-06$ & 25.6513 & Up & tryptophan 2,3-dioxygenase \\
\hline
\end{tabular}




\begin{tabular}{|c|c|c|c|c|c|c|c|}
\hline A_23_P29965 & SMR3B & 1.759372 & $2.76 \mathrm{E}-09$ & $9.04 \mathrm{E}-06$ & 25.54097 & $\mathrm{Up}$ & submaxillary gland androgen regulated protein $3 \mathrm{~B}$ \\
\hline A_23_P167159 & SCRG1 & 2.129388 & $3.06 \mathrm{E}-09$ & $9.29 \mathrm{E}-06$ & 25.23178 & $\mathrm{Up}$ & stimulator of chondrogenesis 1 \\
\hline A_24_P331830 & KAZN & 1.227017 & $3.59 \mathrm{E}-09$ & $1.02 \mathrm{E}-05$ & 24.75271 & $\mathrm{Up}$ & kazrin, periplakin interacting protein \\
\hline A_23_P145514 & IL20RA & 1.535447 & $3.86 \mathrm{E}-09$ & $1.03 \mathrm{E}-05$ & 24.53233 & $\mathrm{Up}$ & interleukin 20 receptor subunit alpha \\
\hline A_23_P328709 & CLRN1 & 1.340457 & $5.2 \mathrm{E}-09$ & $1.27 \mathrm{E}-05$ & 23.67443 & $\mathrm{Up}$ & clarin 1 \\
\hline A_23_P216756 & $\mathrm{FCN} 2$ & 2.077085 & $5.38 \mathrm{E}-09$ & $1.27 \mathrm{E}-05$ & 23.57765 & $\mathrm{Up}$ & ficolin 2 \\
\hline A_24_P709476 & LINC01102 & 1.480536 & $6.97 \mathrm{E}-09$ & $1.56 \mathrm{E}-05$ & 22.85332 & $\mathrm{Up}$ & long intergenic non-protein coding RNA 1102 \\
\hline A_23_P380526 & DPPA4 & 1.231647 & 8.6E-09 & $1.78 \mathrm{E}-05$ & 22.28297 & $\mathrm{Up}$ & $\begin{array}{l}\text { developmental pluripotency associated } 4 \\
\text { serine palmitoyltransferase long chain base }\end{array}$ \\
\hline A_24_P49260 & SPTLC3 & 1.719134 & 8.8E-09 & $1.78 \mathrm{E}-05$ & 22.2211 & $\mathrm{Up}$ & subunit 3 \\
\hline A_23_P430728 & ATP4A & 1.108847 & $1.14 \mathrm{E}-08$ & $2.2 \mathrm{E}-05$ & 21.5462 & $\mathrm{Up}$ & ATPase $\mathrm{H}+/ \mathrm{K}+$ transporting alpha subunit \\
\hline A_33_P3250178 & IQCJ & 1.326735 & $1.73 \mathrm{E}-08$ & $3.2 \mathrm{E}-05$ & 20.48275 & $\mathrm{Up}$ & IQ motif containing $\mathrm{J}$ \\
\hline A_19_P00331971 & MIR503HG & 1.447267 & $2.34 \mathrm{E}-08$ & $4.14 \mathrm{E}-05$ & 19.75227 & $\mathrm{Up}$ & MIR503 host gene \\
\hline A_33_P3236833 & GPR12 & 1.701415 & $2.66 \mathrm{E}-08$ & 4.53E-05 & 19.44286 & $\mathrm{Up}$ & G protein-coupled receptor 12 \\
\hline A_32_P194312 & SDK2 & 1.254756 & 4.19E-08 & $6.6 \mathrm{E}-05$ & 18.40361 & $\mathrm{Up}$ & sidekick cell adhesion molecule 2 \\
\hline A_33_P3234060 & SEPT14 & 1.389704 & 4.95E-08 & $7.45 \mathrm{E}-05$ & 18.03658 & $\mathrm{Up}$ & septin 14 \\
\hline A_33_P3608210 & MIR31HG & 1.48494 & $5.52 \mathrm{E}-08$ & $7.45 \mathrm{E}-05$ & 17.79865 & $\mathrm{Up}$ & MIR31 host gene \\
\hline A_23_P111402 & RSPO3 & 1.785412 & $5.6 \mathrm{E}-08$ & $7.45 \mathrm{E}-05$ & 17.76502 & $\mathrm{Up}$ & R-spondin 3 \\
\hline A_23_P66739 & SLC13A5 & 1.374669 & 7.12E-08 & $9.03 \mathrm{E}-05$ & 17.25669 & $\mathrm{Up}$ & $\begin{array}{l}\text { solute carrier family } 13 \text { member } 5 \\
\text { tubulin polymerization promoting protein family }\end{array}$ \\
\hline A_23_P117464 & ТPРP2 & 1.134536 & 7.49E-08 & $9.03 \mathrm{E}-05$ & 17.15035 & $\mathrm{Up}$ & member 2 \\
\hline A_24_P206624 & FGFR2 & 1.045116 & $7.8 \mathrm{E}-08$ & $9.03 \mathrm{E}-05$ & 17.06439 & $\mathrm{Up}$ & fibroblast growth factor receptor 2 \\
\hline A_33_P3292432 & IQCA1 & 1.083925 & $7.85 \mathrm{E}-08$ & $9.03 \mathrm{E}-05$ & 17.05136 & $\mathrm{Up}$ & IQ motif containing with AAA domain 1 \\
\hline A_23_P36658 & MGST1 & 2.006529 & $8.59 \mathrm{E}-08$ & $9.49 \mathrm{E}-05$ & 16.86567 & $\mathrm{Up}$ & microsomal glutathione S-transferase 1 \\
\hline A_33_P3372954 & LOC387720 & 1.229633 & 8.7E-08 & $9.49 \mathrm{E}-05$ & 16.83959 & $\mathrm{Up}$ & collagen alpha- $1(\mathrm{X})$ chain \\
\hline A_23_P134139 & FABP7 & 1.539766 & $1 \mathrm{E}-07$ & 0.000105 & 16.54886 & $\mathrm{Up}$ & fatty acid binding protein 7 \\
\hline A_23_P215549 & PON3 & 1.382761 & $1.02 \mathrm{E}-07$ & 0.000105 & 16.52506 & $\mathrm{Up}$ & paraoxonase 3 \\
\hline A_23_P374844 & GAL & 1.402474 & $1.07 \mathrm{E}-07$ & 0.000109 & 16.41339 & $\mathrm{Up}$ & galanin and GMAP prepropeptide \\
\hline A_33_P3330149 & PAX6 & 2.074082 & $1.27 \mathrm{E}-07$ & 0.000123 & 16.08292 & $\mathrm{Up}$ & paired box 6 \\
\hline A_33_P3385376 & SYPL2 & 1.302737 & $1.28 \mathrm{E}-07$ & 0.000123 & 16.06335 & $\mathrm{Up}$ & synaptophysin like 2 \\
\hline A_33_P3345344 & DRGX & 1.08197 & $1.33 \mathrm{E}-07$ & 0.000123 & 15.9954 & $\mathrm{Up}$ & dorsal root ganglia homeobox \\
\hline A_23_P127267 & LGI1 & 1.756456 & $1.33 \mathrm{E}-07$ & 0.000123 & 15.9945 & $\mathrm{Up}$ & leucine rich glioma inactivated 1 \\
\hline A_33_P3402329 & MIR503HG & 1.05068 & $1.36 \mathrm{E}-07$ & 0.000123 & 15.94943 & $\mathrm{Up}$ & MIR503 host gene \\
\hline A_33_P3373273 & SLC15A1 & 1.538301 & $1.64 \mathrm{E}-07$ & 0.000142 & 15.58775 & $\mathrm{Up}$ & solute carrier family 15 member 1 \\
\hline A_23_P80162 & TMPRSS3 & 1.596153 & $1.77 \mathrm{E}-07$ & 0.000148 & 15.44677 & $\mathrm{Up}$ & transmembrane protease, serine 3 \\
\hline A_19_P00318205 & \#N/A & 1.577183 & $1.78 \mathrm{E}-07$ & 0.000148 & 15.43499 & $\mathrm{Up}$ & NA \\
\hline A_23_P158318 & ROR2 & 1.473452 & $1.95 \mathrm{E}-07$ & 0.000157 & 15.26237 & $\mathrm{Up}$ & receptor tyrosine kinase like orphan receptor 2 \\
\hline A_24_P289260 & DACT2 & 1.389093 & $1.95 \mathrm{E}-07$ & 0.000157 & 15.25987 & $\mathrm{Up}$ & dishevelled binding antagonist of beta catenin 2 \\
\hline A_32_P111336 & AL591242.1 & 1.506199 & $2.32 \mathrm{E}-07$ & 0.000183 & 14.93773 & $\mathrm{Up}$ & NA \\
\hline A_24_P555066 & SMTNL2 & 1.13931 & $2.61 \mathrm{E}-07$ & 0.000202 & 14.72185 & $\mathrm{Up}$ & smoothelin like 2 \\
\hline A_33_P3310894 & KRT40 & 1.57768 & $2.81 \mathrm{E}-07$ & 0.000208 & 14.59126 & $\mathrm{Up}$ & keratin 40 \\
\hline A_23_P214026 & FBN2 & 1.687663 & $2.83 \mathrm{E}-07$ & 0.000208 & 14.57834 & $\mathrm{Up}$ & fibrillin 2 \\
\hline A_23_P4899 & NTF4 & 1.441871 & $2.84 \mathrm{E}-07$ & 0.000208 & 14.57475 & $\mathrm{Up}$ & neurotrophin 4 \\
\hline A_23_P18672 & GBA3 & 0.964336 & $3.42 \mathrm{E}-07$ & 0.000232 & 14.24096 & $\mathrm{Up}$ & glucosylceramidase beta 3 (gene/pseudogene) \\
\hline
\end{tabular}




\begin{tabular}{|c|c|c|c|c|c|c|c|}
\hline A_19_P00325499 & \#N/A & 0.933759 & $3.44 \mathrm{E}-07$ & 0.000232 & 14.23364 & $\mathrm{Up}$ & NA \\
\hline A_33_P3346073 & SRL & 1.279597 & $3.63 \mathrm{E}-07$ & 0.000238 & 14.13739 & $\mathrm{Up}$ & sarcalumenin \\
\hline A_19_P00321712 & AC112198.2 & 1.208537 & 4.08E-07 & 0.000259 & 13.93544 & $\mathrm{Up}$ & NA \\
\hline A_33_P3411021 & SLIT1 & 1.437893 & 4.23E-07 & 0.000265 & 13.87466 & $\mathrm{Up}$ & slit guidance ligand 1 \\
\hline A_23_P16630 & OR7A5 & 1.409749 & $5.03 \mathrm{E}-07$ & 0.00031 & 13.58235 & $\mathrm{Up}$ & olfactory receptor family 7 subfamily A member 5 \\
\hline A_33_P3249768 & DGKK & 1.719184 & $5.8 \mathrm{E}-07$ & 0.000347 & 13.34618 & $\mathrm{Up}$ & diacylglycerol kinase kappa \\
\hline A_33_P3342797 & LOC401442 & 1.555765 & $6.8 \mathrm{E}-07$ & 0.000396 & 13.08512 & $\mathrm{Up}$ & uncharacterized LOC401442 \\
\hline A_23_P127495 & BBOX1 & 1.969105 & $7.18 \mathrm{E}-07$ & 0.00041 & 12.99661 & $\mathrm{Up}$ & gamma-butyrobetaine hydroxylase 1 \\
\hline A_33_P3723448 & ART3 & 1.310074 & $7.36 \mathrm{E}-07$ & 0.000412 & 12.95714 & $\mathrm{Up}$ & ADP-ribosyltransferase 3 \\
\hline A_33_P3275220 & NRK & 1.438252 & 7.49E-07 & 0.000414 & 12.93021 & $\mathrm{Up}$ & Nik related kinase \\
\hline A_33_P3322400 & LOC654780 & 1.529021 & 8.77E-07 & 0.000472 & 12.67915 & $\mathrm{Up}$ & $\begin{array}{l}\text { splicing factor proline/glutamine-rich } \\
\text { inter-alpha-trypsin inhibitor heavy chain family }\end{array}$ \\
\hline A_33_P3363071 & ITIH6 & 0.970677 & $9.01 \mathrm{E}-07$ & 0.000478 & 12.63721 & $\mathrm{Up}$ & member 6 \\
\hline A_19_P00316371 & AC002480.2 & 1.144117 & $1.1 \mathrm{E}-06$ & 0.000549 & 12.32129 & $\mathrm{Up}$ & NA \\
\hline A_33_P3229370 & ID4 & 1.414675 & $1.11 \mathrm{E}-06$ & 0.000549 & 12.31878 & $\mathrm{Up}$ & inhibitor of DNA binding 4, HLH protein \\
\hline A_33_P3282474 & \#N/A & 1.105066 & $1.11 \mathrm{E}-06$ & 0.000549 & 12.31117 & $\mathrm{Up}$ & NA \\
\hline A_33_P3246026 & DEFB131 & 1.290266 & $1.31 \mathrm{E}-06$ & 0.000615 & 12.05627 & $\mathrm{Up}$ & defensin beta 131 \\
\hline A_33_P3400763 & PLIN4 & 1.033075 & $1.33 \mathrm{E}-06$ & 0.000616 & 12.03503 & $\mathrm{Up}$ & perilipin 4 \\
\hline A_33_P3504002 & LINC01299 & 1.129723 & $1.57 \mathrm{E}-06$ & 0.000697 & 11.7883 & $\mathrm{Up}$ & long intergenic non-protein coding RNA 1299 \\
\hline A_32_P324933 & LINC00282 & 0.971613 & $1.72 \mathrm{E}-06$ & 0.000748 & 11.65582 & $\mathrm{Up}$ & long intergenic non-protein coding RNA 282 \\
\hline A_33_P3248799 & TRDN & 2.047985 & $1.75 \mathrm{E}-06$ & 0.000749 & 11.63034 & $\mathrm{Up}$ & triadin \\
\hline A_23_P311895 & CLIC5 & 1.76 & $1.86 \mathrm{E}-06$ & 0.000777 & 11.54202 & $\mathrm{Up}$ & chloride intracellular channel 5 \\
\hline A_33_P3391915 & BTBD17 & 1.379368 & $1.97 \mathrm{E}-06$ & 0.000812 & 11.45968 & $\mathrm{Up}$ & BTB domain containing 17 \\
\hline A_33_P3315134 & DIRC3 & 1.187589 & $2.04 \mathrm{E}-06$ & 0.000812 & 11.41367 & $\mathrm{Up}$ & disrupted in renal carcinoma 3 \\
\hline A_33_P3336257 & IRX1 & 1.782642 & $2.19 \mathrm{E}-06$ & 0.000854 & 11.31183 & $\mathrm{Up}$ & iroquoishomeobox 1 \\
\hline A_33_P3316953 & RBFOX3 & 2.121609 & $2.25 \mathrm{E}-06$ & 0.000869 & 11.27381 & $\mathrm{Up}$ & RNA binding protein, fox-1 homolog 3 \\
\hline A_23_P103765 & FCER1A & 1.675704 & $2.5 \mathrm{E}-06$ & 0.000926 & 11.12106 & $\mathrm{Up}$ & Fc fragment of IgE receptor Ia \\
\hline A_33_P3240094 & NGEF & 1.065886 & $2.66 \mathrm{E}-06$ & 0.000969 & 11.03844 & $\mathrm{Up}$ & neuronal guanine nucleotide exchange factor \\
\hline A_23_P162010 & CCKBR & 1.368159 & $2.67 \mathrm{E}-06$ & 0.000969 & 11.03391 & $\mathrm{Up}$ & cholecystokinin B receptor \\
\hline A_23_P95790 & ITLN1 & 1.534417 & $2.72 \mathrm{E}-06$ & 0.00098 & 11.00679 & $\mathrm{Up}$ & intelectin 1 \\
\hline A_23_P383227 & S100A1 & 1.861029 & $2.85 \mathrm{E}-06$ & 0.001006 & 10.94318 & $\mathrm{Up}$ & S100 calcium binding protein A1 \\
\hline A_23_P42909 & TMEM139 & 1.402909 & $2.87 \mathrm{E}-06$ & 0.001006 & 10.93278 & Up & transmembrane protein 139 \\
\hline A_23_P15889 & CBLN2 & 1.141876 & $2.89 \mathrm{E}-06$ & 0.001006 & 10.92424 & $\mathrm{Up}$ & cerebellin 2 precursor \\
\hline A_33_P3619171 & PMAIP1 & 1.187979 & $3.01 \mathrm{E}-06$ & 0.001041 & 10.86654 & $\mathrm{Up}$ & phorbol-12-myristate-13-acetate-induced protein 1 \\
\hline A_23_P8497 & GHRHR & 1.170394 & 3.07E-06 & 0.001054 & 10.83768 & $\mathrm{Up}$ & growth hormone releasing hormone receptor \\
\hline A_33_P3270863 & $\mathrm{XDH}$ & 1.809518 & $3.15 \mathrm{E}-06$ & 0.001071 & 10.80343 & $\mathrm{Up}$ & xanthine dehydrogenase \\
\hline A_24_P595717 & TOGARAM2 & 1.604949 & $3.4 \mathrm{E}-06$ & 0.001139 & 10.69969 & $\mathrm{Up}$ & TOG array regulator of axonemal microtubules 2 \\
\hline A_33_P3350164 & $\mathrm{CCDC} 178$ & 1.42881 & $3.44 \mathrm{E}-06$ & 0.001144 & 10.68331 & $\mathrm{Up}$ & coiled-coil domain containing 178 \\
\hline A_23_P203267 & TRIM29 & 1.443956 & $3.54 \mathrm{E}-06$ & 0.001159 & 10.64492 & $\mathrm{Up}$ & $\begin{array}{l}\text { tripartite motif containing } 29 \\
\text { UDP glucuronosyltransferase family } 2 \text { member }\end{array}$ \\
\hline A_33_P3389237 & UGT2A2 & 1.479469 & 3.6E-06 & 0.00117 & 10.62203 & $\mathrm{Up}$ & A2 \\
\hline A_33_P3412262 & JAKMIP2-AS1 & 1.32862 & 3.67E-06 & 0.001183 & 10.59731 & Up & JAKMIP2 antisense RNA 1 \\
\hline A_24_P27234 & SOX5 & 1.659349 & $3.82 \mathrm{E}-06$ & 0.001212 & 10.54464 & $\mathrm{Up}$ & SRY-box 5 \\
\hline A_33_P3409086 & S100A1 & 1.494024 & 4.14E-06 & 0.001285 & 10.43768 & $\mathrm{Up}$ & S100 calcium binding protein A1 \\
\hline
\end{tabular}




\begin{tabular}{|c|c|c|c|c|c|c|c|}
\hline A_32_P3476 & RPRML & 1.611243 & 4.32E-06 & 0.001322 & 10.38091 & $\mathrm{Up}$ & reprimo like \\
\hline A_33_P3773374 & AC120036.5 & 1.753756 & $4.49 \mathrm{E}-06$ & 0.001353 & 10.33104 & $\mathrm{Up}$ & NA \\
\hline A_23_P153155 & GALR1 & 1.619947 & 4.67E-06 & 0.001358 & 10.27926 & $\mathrm{Up}$ & galanin receptor 1 \\
\hline A_23_P83134 & GAS1 & 1.058861 & $4.68 \mathrm{E}-06$ & 0.001358 & 10.27589 & $\mathrm{Up}$ & growth arrest specific 1 \\
\hline A_33_P3308964 & PPP1R17 & 1.085168 & $4.71 \mathrm{E}-06$ & 0.001358 & 10.26698 & $\mathrm{Up}$ & protein phosphatase 1 regulatory subunit 17 \\
\hline A_23_P302060 & IFNE & 0.935355 & $4.82 \mathrm{E}-06$ & 0.001371 & 10.23827 & $\mathrm{Up}$ & interferon epsilon \\
\hline A_23_P132378 & CELSR1 & 1.253634 & 4.83E-06 & 0.001371 & 10.23304 & $\mathrm{Up}$ & cadherin EGF LAG seven-pass G-type receptor 1 \\
\hline A_23_P29975 & C4orf19 & 1.051466 & $5.25 \mathrm{E}-06$ & 0.001459 & 10.12693 & $\mathrm{Up}$ & chromosome 4 open reading frame 19 \\
\hline A_33_P3345036 & POMC & 1.014551 & $5.73 \mathrm{E}-06$ & 0.001563 & 10.01362 & $\mathrm{Up}$ & proopiomelanocortin \\
\hline A_19_P00315473 & \#N/A & 1.012926 & $6.47 \mathrm{E}-06$ & 0.00171 & 9.859854 & $\mathrm{Up}$ & NA \\
\hline A_24_P365721 & SLC6A14 & 1.636424 & $6.82 \mathrm{E}-06$ & 0.00179 & 9.794187 & $\mathrm{Up}$ & $\begin{array}{l}\text { solute carrier family } 6 \text { member } 14 \\
\text { lecithin retinol acyltransferase }\end{array}$ \\
\hline A_32_P113066 & LRAT & 1.318608 & $6.87 \mathrm{E}-06$ & 0.001793 & 9.784901 & $\mathrm{Up}$ & $\begin{array}{l}\text { (phosphatidylcholine--retinol O-acyltransferase) } \\
\text { serine palmitoyltransferase long chain base }\end{array}$ \\
\hline A_33_P3415395 & SPTLC3 & 1.309174 & 7.32E-06 & 0.00185 & 9.704786 & Up & subunit 3 \\
\hline A_23_P101505 & KLK11 & 1.847661 & 7.34E-06 & 0.00185 & 9.702092 & $\mathrm{Up}$ & kallikrein related peptidase 11 \\
\hline A_32_P41604 & F5 & 1.535516 & 7.35E-06 & 0.00185 & 9.700312 & $\mathrm{Up}$ & coagulation factor $\mathrm{V}$ \\
\hline A_33_P3367196 & CNTNAP2 & 1.605568 & 7.42E-06 & 0.001856 & 9.688935 & $\mathrm{Up}$ & contactin associated protein-like 2 \\
\hline A_33_P3215640 & PI16 & 1.290311 & 7.68E-06 & 0.001911 & 9.645508 & $\mathrm{Up}$ & $\begin{array}{l}\text { peptidase inhibitor } 16 \\
\text { ectonucleotidepyrophosphatase/phosphodiesterase }\end{array}$ \\
\hline A_24_P397255 & ENPP6 & 1.594549 & $8.75 \mathrm{E}-06$ & 0.002116 & 9.485334 & $\mathrm{Up}$ & 6 \\
\hline A_33_P3217437 & GBP6 & 1.036074 & $9.63 \mathrm{E}-06$ & 0.002289 & 9.369347 & $\mathrm{Up}$ & guanylate binding protein family member 6 \\
\hline A_33_P3351264 & OVOL3 & 1.050788 & $9.78 \mathrm{E}-06$ & 0.002311 & 9.351444 & $\mathrm{Up}$ & ovo like zinc finger 3 \\
\hline A_23_P112327 & $\mathrm{OBP} 2 \mathrm{~A}$ & 1.350445 & $1 \mathrm{E}-05$ & 0.002352 & 9.323343 & $\mathrm{Up}$ & odorant binding protein $2 \mathrm{~A}$ \\
\hline A_23_P75283 & RBP4 & 1.726183 & $1.04 \mathrm{E}-05$ & 0.00242 & 9.282412 & $\mathrm{Up}$ & retinol binding protein 4 \\
\hline A_33_P3252048 & PPP1R27 & 1.719928 & $1.05 \mathrm{E}-05$ & 0.002445 & 9.263686 & $\mathrm{Up}$ & protein phosphatase 1 regulatory subunit 27 \\
\hline A_23_P202053 & ITIH2 & 1.137137 & $1.1 \mathrm{E}-05$ & 0.002526 & 9.21207 & $\mathrm{Up}$ & inter-alpha-trypsin inhibitor heavy chain 2 \\
\hline A_23_P328740 & NEURL3 & 1.378209 & $1.16 \mathrm{E}-05$ & 0.002615 & 9.144984 & $\mathrm{Up}$ & neuralized E3 ubiquitin protein ligase 3 \\
\hline A_23_P416774 & CLIC5 & 1.122275 & $1.19 \mathrm{E}-05$ & 0.002615 & 9.114478 & $\mathrm{Up}$ & chloride intracellular channel 5 \\
\hline A_24_P13024 & SLC16A12 & 1.528046 & $1.2 \mathrm{E}-05$ & 0.002615 & 9.110565 & $\mathrm{Up}$ & solute carrier family 16 member 12 \\
\hline A_33_P3287472 & PPP1R27 & 1.441994 & $1.23 \mathrm{E}-05$ & 0.002663 & 9.081115 & $\mathrm{Up}$ & protein phosphatase 1 regulatory subunit 27 \\
\hline A_23_P69738 & RASL11B & 1.2602 & $1.25 \mathrm{E}-05$ & 0.002678 & 9.056703 & $\mathrm{Up}$ & $\begin{array}{l}\text { RAS like family } 11 \text { member B } \\
\text { potassium voltage-gated channel subfamily } \mathrm{J}\end{array}$ \\
\hline A_24_P339429 & $\mathrm{KCNJ} 12$ & 1.592216 & $1.33 \mathrm{E}-05$ & 0.002794 & 8.983141 & $\mathrm{Up}$ & member 12 \\
\hline A_33_P3420757 & AQP4 & 1.278225 & $1.34 \mathrm{E}-05$ & 0.002794 & 8.978342 & Up & aquaporin 4 \\
\hline A_23_P159335 & CD8B & 0.96392 & $1.37 \mathrm{E}-05$ & 0.002835 & 8.949807 & $\mathrm{Up}$ & CD8b molecule \\
\hline A_23_P24903 & P2RY2 & 0.995063 & $1.44 \mathrm{E}-05$ & 0.002894 & 8.898402 & $\mathrm{Up}$ & purinergic receptor $\mathrm{P} 2 \mathrm{Y} 2$ \\
\hline A_23_P62607 & IL22RA1 & 1.500212 & $1.48 \mathrm{E}-05$ & 0.002928 & 8.862964 & $\mathrm{Up}$ & interleukin 22 receptor subunit alpha 1 \\
\hline A_33_P3304533 & RNF207 & 1.223159 & $1.67 \mathrm{E}-05$ & 0.003222 & 8.722137 & $\mathrm{Up}$ & $\begin{array}{l}\text { ring finger protein } 207 \\
\text { ADAM metallopeptidase with thrombospondin }\end{array}$ \\
\hline A_32_P34844 & ADAMTS18 & 1.549798 & $1.79 \mathrm{E}-05$ & 0.003372 & 8.645032 & Up & type 1 motif 18 \\
\hline A_23_P389897 & NGFR & 1.465734 & $1.8 \mathrm{E}-05$ & 0.003372 & 8.640153 & $\mathrm{Up}$ & $\begin{array}{l}\text { nerve growth factor receptor } \\
\text { potassium voltage-gated channel subfamily } \mathrm{J}\end{array}$ \\
\hline A_23_P501193 & KCNJ16 & 1.649162 & $1.81 \mathrm{E}-05$ & 0.003381 & 8.632189 & $\mathrm{Up}$ & $\begin{array}{l}\text { member } 16 \\
\text { EGF containing fibulin like extracellular matrix }\end{array}$ \\
\hline A_23_P501007 & EFEMP1 & 0.963358 & $1.86 \mathrm{E}-05$ & 0.003434 & 8.604817 & $\mathrm{Up}$ & protein 1 \\
\hline A_33_P3353027 & CHN2 & 0.959558 & $1.92 \mathrm{E}-05$ & 0.003523 & 8.566558 & $\mathrm{Up}$ & chimerin 2 \\
\hline A_23_P47181 & GPHA2 & 1.258662 & $1.95 \mathrm{E}-05$ & 0.003562 & 8.549199 & $\mathrm{Up}$ & glycoprotein hormone alpha 2 \\
\hline
\end{tabular}




\begin{tabular}{|c|c|c|c|c|c|c|c|}
\hline A_33_P3232655 & GHRHR & 1.32053 & $2.15 \mathrm{E}-05$ & 0.003813 & 8.440624 & $\mathrm{Up}$ & growth hormone releasing hormone receptor \\
\hline A_24_P280333 & KRT40 & 1.503725 & 2.2E-05 & 0.003863 & 8.416969 & $\mathrm{Up}$ & keratin 40 \\
\hline A_32_P218355 & C6orf132 & 1.411295 & $2.3 \mathrm{E}-05$ & 0.003978 & 8.367301 & $\mathrm{Up}$ & chromosome 6 open reading frame 132 \\
\hline A_23_P402765 & NRAP & 1.811676 & $2.31 \mathrm{E}-05$ & 0.003978 & 8.362425 & $\mathrm{Up}$ & nebulin related anchoring protein \\
\hline A_33_P3250173 & IQCJ & 1.201705 & $2.32 \mathrm{E}-05$ & 0.003978 & 8.357898 & $\mathrm{Up}$ & IQ motif containing $\mathrm{J}$ \\
\hline A_24_P726336 & PHACTR2 & 0.937904 & $2.47 \mathrm{E}-05$ & 0.0042 & 8.289587 & $\mathrm{Up}$ & phosphatase and actin regulator 2 \\
\hline A_33_P3319880 & $\mathrm{EVC} 2$ & 1.047398 & $2.51 \mathrm{E}-05$ & 0.004259 & 8.269911 & $\mathrm{Up}$ & EvCciliary complex subunit 2 \\
\hline A_33_P3355508 & FOXL2 & 1.219617 & $2.54 \mathrm{E}-05$ & 0.004275 & 8.257205 & $\mathrm{Up}$ & forkhead box L2 \\
\hline A_24_P385732 & SLC51A & 1.228643 & 2.6E-05 & 0.004347 & 8.234886 & $\mathrm{Up}$ & solute carrier family 51 alpha subunit \\
\hline A_19_P00320141 & NR2F1-AS1 & 1.049334 & 2.63E-05 & 0.004388 & 8.220529 & $\mathrm{Up}$ & NR2F1 antisense RNA 1 \\
\hline A_23_P166269 & FAM3B & 1.658734 & $2.81 \mathrm{E}-05$ & 0.004546 & 8.14888 & $\mathrm{Up}$ & family with sequence similarity 3 member B \\
\hline A_24_P236251 & DLK1 & 1.084201 & 2.9E-05 & 0.004667 & 8.116486 & $\mathrm{Up}$ & delta like non-canonical Notch ligand 1 \\
\hline A_24_P50368 & BLID & 0.934055 & 3.01E-05 & 0.004764 & 8.075302 & $\mathrm{Up}$ & BH3-like motif containing, cell death inducer \\
\hline A_23_P43197 & CALB1 & 1.816152 & $3.01 \mathrm{E}-05$ & 0.004764 & 8.074442 & $\mathrm{Up}$ & $\begin{array}{l}\text { calbindin } 1 \\
\text { UDP glucuronosyltransferase family } 2 \text { member }\end{array}$ \\
\hline A_33_P3346766 & UGT2A1 & 1.919609 & $3.08 \mathrm{E}-05$ & 0.004817 & 8.050798 & $\mathrm{Up}$ & A1 complex locus \\
\hline A_19_P00322810 & \#N/A & 0.976388 & $3.19 \mathrm{E}-05$ & 0.004888 & 8.012101 & $\mathrm{Up}$ & NA \\
\hline A_33_P3279590 & OGN & 1.376373 & $3.24 \mathrm{E}-05$ & 0.004921 & 7.998292 & $\mathrm{Up}$ & osteoglycin \\
\hline A_23_P212508 & $\mathrm{TF}$ & 1.475208 & $3.38 \mathrm{E}-05$ & 0.005067 & 7.952258 & $\mathrm{Up}$ & transferrin \\
\hline A_24_P655849 & SMAD9 & 1.578849 & $3.42 \mathrm{E}-05$ & 0.00508 & 7.939515 & $\mathrm{Up}$ & SMAD family member 9 \\
\hline A_19_P00801466 & LINC00598 & 1.475444 & $3.45 \mathrm{E}-05$ & 0.00508 & 7.930302 & $\mathrm{Up}$ & long intergenic non-protein coding RNA 598 \\
\hline A_23_P401055 & SOX2 & 1.69957 & $3.47 \mathrm{E}-05$ & 0.005086 & 7.925451 & $\mathrm{Up}$ & SRY-box 2 \\
\hline A_23_P500010 & KLK12 & 2.06498 & $3.51 \mathrm{E}-05$ & 0.005129 & 7.913013 & $\mathrm{Up}$ & kallikrein related peptidase 12 \\
\hline A_24_P882732 & KRT17P1 & 1.106234 & 3.67E-05 & 0.005299 & 7.864481 & $\mathrm{Up}$ & $\begin{array}{l}\text { keratin } 17 \text { pseudogene } 1 \\
\text { somatomedin } \mathrm{B} \text { and thrombospondin type } 1\end{array}$ \\
\hline A_23_P170649 & SBSPON & 1.314417 & $3.84 \mathrm{E}-05$ & 0.005496 & 7.817453 & $\mathrm{Up}$ & domain containing \\
\hline A_33_P3345534 & KRT14 & 1.187608 & $3.91 \mathrm{E}-05$ & 0.00555 & 7.798667 & $\mathrm{Up}$ & keratin 14 \\
\hline A_32_P930375 & DLGAP2 & 1.302276 & $3.98 \mathrm{E}-05$ & 0.005631 & 7.780067 & $\mathrm{Up}$ & DLG associated protein 2 \\
\hline A_23_P110319 & CWH43 & 1.018626 & 4.1E-05 & 0.005709 & 7.750289 & $\mathrm{Up}$ & cell wall biogenesis $43 \mathrm{C}$-terminal homolog \\
\hline A_23_P334798 & LRRC2 & 0.953096 & $4.18 \mathrm{E}-05$ & 0.005713 & 7.731307 & $\mathrm{Up}$ & leucine rich repeat containing 2 \\
\hline A_33_P3347397 & AMOT & 1.359913 & $4.28 \mathrm{E}-05$ & 0.005822 & 7.705274 & $\mathrm{Up}$ & angiomotin \\
\hline A_19_P00318158 & \#N/A & 1.004498 & $4.39 \mathrm{E}-05$ & 0.005857 & 7.67955 & $\mathrm{Up}$ & NA \\
\hline A_33_P3340981 & LINC00598 & 1.256571 & 4.45E-05 & 0.005905 & 7.666631 & $\mathrm{Up}$ & long intergenic non-protein coding RNA 598 \\
\hline A_33_P3340580 & ZNF385C & 0.953391 & 4.66E-05 & 0.006119 & 7.618782 & $\mathrm{Up}$ & zinc finger protein $385 \mathrm{C}$ \\
\hline A_23_P425681 & $\mathrm{CCK}$ & 1.622521 & $4.79 \mathrm{E}-05$ & 0.006272 & 7.590454 & $\mathrm{Up}$ & cholecystokinin \\
\hline A_23_P63209 & HSD11B1 & 1.715094 & 4.83E-05 & 0.006272 & 7.583273 & Up & hydroxysteroid 11-beta dehydrogenase 1 \\
\hline A_23_P9711 & HS3ST4 & 1.269685 & 4.84E-05 & 0.006272 & 7.581112 & Up & heparansulfate-glucosamine 3 -sulfotransferase 4 \\
\hline A_24_P691826 & WFDC21P & 0.992875 & $5.11 \mathrm{E}-05$ & 0.00653 & 7.524302 & $\mathrm{Up}$ & WAP four-disulfide core domain 21 , pseudogene \\
\hline A_19_P00807452 & \#N/A & 1.104671 & $5.13 \mathrm{E}-05$ & 0.00653 & 7.520302 & $\mathrm{Up}$ & NA \\
\hline A_33_P3267375 & SGCD & 1.257873 & $5.28 \mathrm{E}-05$ & 0.006685 & 7.492188 & Up & sarcoglycan delta \\
\hline A_33_P3329088 & PRSS8 & 1.069842 & $5.57 \mathrm{E}-05$ & 0.00695 & 7.439292 & $\mathrm{Up}$ & $\begin{array}{l}\text { protease, serine } 8 \\
\text { olfactory receptor family } 3 \text { subfamily A member } 4\end{array}$ \\
\hline A_33_P3249229 & OR3A4P & 1.184305 & $5.63 \mathrm{E}-05$ & 0.006981 & 7.427986 & $\mathrm{Up}$ & pseudogene \\
\hline A_32_P85106 & C6orf118 & 1.123262 & $5.67 \mathrm{E}-05$ & 0.007016 & 7.420158 & $\mathrm{Up}$ & chromosome 6 open reading frame 118 \\
\hline A_19_P00804546 & MIR503HG & 2.046985 & $6.03 \mathrm{E}-05$ & 0.007246 & 7.359333 & $\mathrm{Up}$ & MIR503 host gene \\
\hline
\end{tabular}




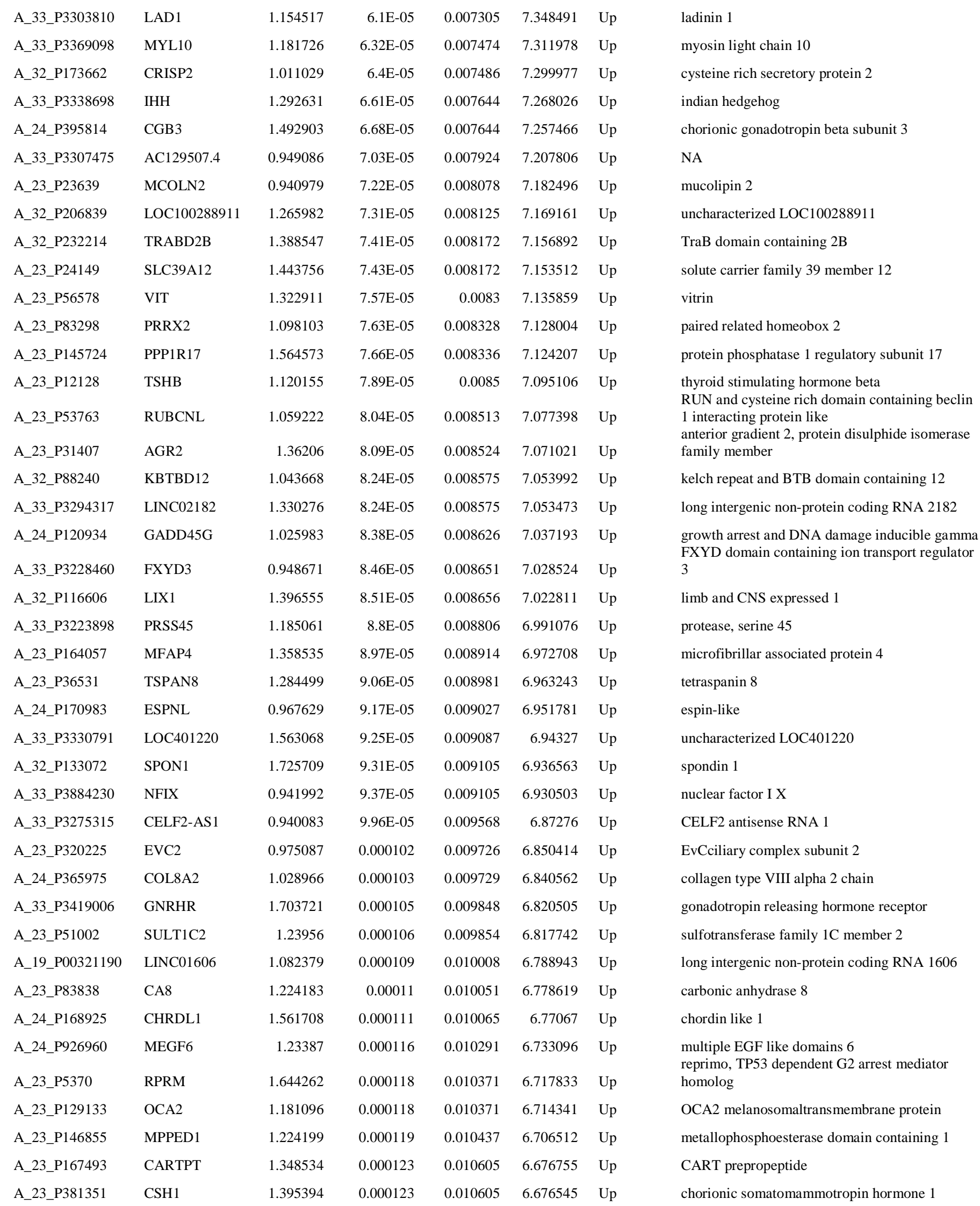




\begin{tabular}{|c|c|c|c|c|c|c|c|}
\hline A_23_P252462 & GALNT9 & 0.972962 & 0.000126 & 0.01073 & 6.651804 & $\mathrm{Up}$ & polypeptide $\mathrm{N}$-acetylgalactosaminyltransferase 9 \\
\hline A_23_P82990 & OGN & 1.458835 & 0.000129 & 0.010881 & 6.628807 & $\mathrm{Up}$ & osteoglycin \\
\hline A_32_P84369 & FAM153B & 1.100172 & 0.000131 & 0.010897 & 6.618167 & $\mathrm{Up}$ & family with sequence similarity 153 member B \\
\hline A_33_P3252394 & GADD45G & 1.041451 & 0.000132 & 0.010919 & 6.612967 & $\mathrm{Up}$ & growth arrest and DNA damage inducible gamma \\
\hline A_23_P209978 & VSNL1 & 1.042135 & 0.000134 & 0.011037 & 6.599626 & $\mathrm{Up}$ & visinin like 1 \\
\hline A_33_P3231357 & KISS1R & 1.447717 & 0.000134 & 0.01105 & 6.596694 & $\mathrm{Up}$ & KISS1 receptor \\
\hline A_23_P212830 & FGFR3 & 1.121697 & 0.000135 & 0.011086 & 6.586685 & $\mathrm{Up}$ & fibroblast growth factor receptor 3 \\
\hline A_33_P3680789 & PROSER2-AS1 & 1.087472 & 0.000137 & 0.011165 & 6.575783 & $\mathrm{Up}$ & PROSER2 antisense RNA 1 \\
\hline A_33_P3341499 & WNT5A & 1.252562 & 0.000137 & 0.011165 & 6.574844 & $\mathrm{Up}$ & Wnt family member $5 \mathrm{~A}$ \\
\hline A_23_P357881 & CD8B & 1.210532 & 0.000138 & 0.011213 & 6.569217 & $\mathrm{Up}$ & CD8b molecule \\
\hline A_23_P81103 & SFRP2 & 1.416148 & 0.000152 & 0.011906 & 6.483851 & $\mathrm{Up}$ & secreted frizzled related protein 2 \\
\hline A_23_P92730 & HSPB3 & 1.413693 & 0.000162 & 0.012387 & 6.426669 & $\mathrm{Up}$ & heat shock protein family B (small) member 3 \\
\hline A_33_P3306267 & PAX6 & 1.112687 & 0.000163 & 0.012452 & 6.416123 & $\mathrm{Up}$ & paired box 6 \\
\hline A_24_P345451 & CYBRD1 & 1.038518 & 0.000166 & 0.01256 & 6.402632 & $\mathrm{Up}$ & $\begin{array}{l}\text { cytochrome } b \text { reductase } 1 \\
\text { FXYD domain containing ion transport regulator }\end{array}$ \\
\hline A_33_P3228450 & FXYD3 & 1.266019 & 0.000169 & 0.012624 & 6.387741 & $\mathrm{Up}$ & 3 \\
\hline A_33_P3480395 & FLJ30901 & 1.022724 & 0.000172 & 0.012732 & 6.371786 & $\mathrm{Up}$ & uncharacterized protein FLJ30901 \\
\hline A_23_P138938 & PGR & 1.816923 & 0.000175 & 0.012856 & 6.35462 & $\mathrm{Up}$ & progesterone receptor \\
\hline A_19_P00803588 & \#N/A & 1.00275 & 0.000176 & 0.012892 & 6.349834 & $\mathrm{Up}$ & NA \\
\hline A_23_P118065 & HSD17B2 & 1.040626 & 0.000183 & 0.013153 & 6.316724 & $\mathrm{Up}$ & hydroxysteroid 17-beta dehydrogenase 2 \\
\hline A_32_P160563 & OPCML & 1.365608 & 0.000185 & 0.01326 & 6.308043 & $\mathrm{Up}$ & opioid binding protein/cell adhesion molecule like \\
\hline A_23_P317760 & FAM217A & 0.982831 & 0.000189 & 0.013446 & 6.284687 & $\mathrm{Up}$ & family with sequence similarity 217 member A \\
\hline A_23_P76332 & RFX4 & 1.06413 & 0.000193 & 0.013638 & 6.266747 & $\mathrm{Up}$ & $\begin{array}{l}\text { regulatory factor } \mathrm{X} 4 \\
\text { anterior gradient } 3 \text {, protein disulphide isomerase }\end{array}$ \\
\hline A_23_P42811 & AGR3 & 1.186616 & 0.000196 & 0.013804 & 6.253129 & $\mathrm{Up}$ & family member \\
\hline A_24_P940644 & SLIT1 & 1.673787 & 0.000201 & 0.013821 & 6.233263 & $\mathrm{Up}$ & slit guidance ligand 1 \\
\hline A_23_P26847 & SOX9 & 1.113591 & 0.000204 & 0.01395 & 6.220577 & $\mathrm{Up}$ & SRY-box 9 \\
\hline A_33_P3351681 & DEUP1 & 1.483472 & 0.00021 & 0.014139 & 6.195544 & $\mathrm{Up}$ & deuterosome assembly protein 1 \\
\hline A_23_P344408 & CSHL1 & 1.2167 & 0.000215 & 0.014376 & 6.173456 & $\mathrm{Up}$ & chorionic somatomammotropin hormone like 1 \\
\hline A_32_P111639 & CHST9 & 1.181236 & 0.000222 & 0.014537 & 6.146767 & $\mathrm{Up}$ & carbohydrate sulfotransferase 9 \\
\hline A_33_P3248405 & NRK & 1.148294 & 0.000223 & 0.014561 & 6.141927 & $\mathrm{Up}$ & Nik related kinase \\
\hline A_33_P3245238 & SULT1C2 & 1.01799 & 0.000226 & 0.014718 & 6.130746 & $\mathrm{Up}$ & sulfotransferase family $1 \mathrm{C}$ member 2 \\
\hline A_23_P75063 & DYDC2 & 1.077759 & 0.00023 & 0.014886 & 6.113345 & $\mathrm{Up}$ & DPY30 domain containing 2 \\
\hline A_33_P3410121 & CFAP74 & 1.034589 & 0.000234 & 0.015035 & 6.098098 & $\mathrm{Up}$ & cilia and flagella associated protein 74 \\
\hline A_23_P39605 & SULT1C4 & 1.072548 & 0.000236 & 0.015056 & 6.090655 & $\mathrm{Up}$ & sulfotransferase family $1 \mathrm{C}$ member 4 \\
\hline A_23_P251132 & SNTG2 & 1.884616 & 0.000237 & 0.015081 & 6.086334 & $\mathrm{Up}$ & syntrophin gamma 2 \\
\hline A_33_P3423420 & ZNF750 & 1.064614 & 0.000239 & 0.015087 & 6.082126 & $\mathrm{Up}$ & zinc finger protein 750 \\
\hline A_33_P3258046 & TAC4 & 1.141906 & 0.000242 & 0.015181 & 6.071276 & $\mathrm{Up}$ & tachykinin 4 (hemokinin) \\
\hline A_23_P136116 & AGMO & 1.074353 & 0.000242 & 0.015181 & 6.070326 & $\mathrm{Up}$ & alkylglycerolmonooxygenase \\
\hline A_33_P3317253 & PTER & 1.057567 & 0.000247 & 0.015377 & 6.050692 & $\mathrm{Up}$ & phosphotriesterase related \\
\hline A_23_P431139 & CTRB1 & 1.081155 & 0.000249 & 0.015428 & 6.043694 & $\mathrm{Up}$ & chymotrypsinogen B1 \\
\hline A_23_P18282 & DLEC1 & 1.106803 & 0.000255 & 0.015611 & 6.024776 & $\mathrm{Up}$ & deleted in lung and esophageal cancer 1 \\
\hline A_23_P31124 & COL21A1 & 1.493919 & 0.000258 & 0.015701 & 6.015768 & $\mathrm{Up}$ & collagen type XXI alpha 1 chain \\
\hline A_19_P00320685 & LINC01852 & 0.977693 & 0.000258 & 0.015701 & 6.013804 & $\mathrm{Up}$ & long intergenic non-protein coding RNA 1852 \\
\hline
\end{tabular}




\begin{tabular}{|c|c|c|c|c|c|c|c|}
\hline A_19_P00805833 & ATF7IP2 & 1.079212 & 0.000259 & 0.015703 & 6.010456 & Up & $\begin{array}{l}\text { activating transcription factor } 7 \text { interacting protein } \\
2\end{array}$ \\
\hline A_33_P3249534 & NEFM & 1.748709 & 0.000266 & 0.015898 & 5.989561 & $\mathrm{Up}$ & neurofilament medium \\
\hline A_19_P00316370 & AC002480.2 & 1.245626 & 0.000277 & 0.016233 & 5.953808 & $\mathrm{Up}$ & NA \\
\hline A_23_P124619 & S100A14 & 1.025455 & 0.000278 & 0.016296 & 5.94929 & $\mathrm{Up}$ & S100 calcium binding protein A14 \\
\hline A_33_P3354414 & AOX1 & 1.169692 & 0.000283 & 0.016493 & 5.934365 & $\mathrm{Up}$ & aldehyde oxidase 1 \\
\hline A_32_P158966 & KLRF1 & 0.936194 & 0.000285 & 0.016572 & 5.92911 & $\mathrm{Up}$ & killer cell lectin like receptor F1 \\
\hline A_33_P3352906 & KCNIP4-IT1 & 1.113185 & 0.000289 & 0.016663 & 5.916334 & $\mathrm{Up}$ & KCNIP4 intronic transcript 1 \\
\hline A_33_P3420224 & ENTPD8 & 0.994821 & 0.000296 & 0.016851 & 5.896821 & $\mathrm{Up}$ & ectonucleoside triphosphate diphosphohydrolase 8 \\
\hline A_23_P363255 & CCDC68 & 1.63892 & 0.000298 & 0.016851 & 5.892889 & $\mathrm{Up}$ & coiled-coil domain containing 68 \\
\hline A_33_P3297562 & IRX2 & 0.941375 & 0.000298 & 0.016851 & 5.89031 & $\mathrm{Up}$ & iroquoishomeobox 2 \\
\hline A_33_P3368646 & CNKSR3 & 1.102873 & 0.000303 & 0.016944 & 5.878301 & $\mathrm{Up}$ & CNKSR family member 3 \\
\hline A_23_P77731 & CRYM & 0.956236 & 0.000309 & 0.017112 & 5.860011 & Up & crystallin mu \\
\hline A_33_P3298810 & FFAR3 & 1.175134 & 0.000311 & 0.017186 & 5.855259 & $\mathrm{Up}$ & free fatty acid receptor 3 \\
\hline A_33_P3423874 & MYZAP & 1.382209 & 0.000315 & 0.017267 & 5.845554 & Up & myocardial zonulaadherens protein \\
\hline A_23_P81590 & PDE6A & 1.325565 & 0.000317 & 0.017267 & 5.840506 & Up & phosphodiesterase $6 \mathrm{~A}$ \\
\hline A_23_P118615 & ABCA8 & 1.154317 & 0.000335 & 0.017778 & 5.793871 & $\mathrm{Up}$ & ATP binding cassette subfamily A member 8 \\
\hline A_19_P00317324 & \#N/A & 1.111694 & 0.000336 & 0.017778 & 5.791414 & Up & NA \\
\hline A_23_P122924 & INHBA & 0.971104 & 0.000336 & 0.017778 & 5.790115 & $\mathrm{Up}$ & $\begin{array}{l}\text { inhibin beta A subunit } \\
\text { low density lipoprotein receptor class A domain }\end{array}$ \\
\hline A_33_P3260125 & LDLRAD2 & 1.408377 & 0.000337 & 0.017778 & 5.78717 & Up & containing 2 \\
\hline A_33_P3281667 & CNTNAP3 & 0.947904 & 0.000339 & 0.017833 & 5.781984 & $\mathrm{Up}$ & $\begin{array}{l}\text { contactin associated protein-like } 3 \\
\text { family with sequence similarity } 153 \text {, member C, }\end{array}$ \\
\hline A_32_P84373 & FAM153C & 1.604174 & 0.000353 & 0.018266 & 5.74907 & Up & pseudogene \\
\hline A_33_P3847514 & C6orf141 & 1.000679 & 0.000353 & 0.018268 & 5.74794 & $\mathrm{Up}$ & chromosome 6 open reading frame 141 \\
\hline A_24_P34155 & RUNX1 & 1.245442 & 0.00036 & 0.01843 & 5.732384 & $\mathrm{Up}$ & runt related transcription factor 1 \\
\hline A_33_P3354429 & TRIM71 & 0.994075 & 0.000367 & 0.018648 & 5.715808 & $\mathrm{Up}$ & tripartite motif containing 71 \\
\hline A_23_P100642 & PNMT & 1.853774 & 0.000367 & 0.018648 & 5.715693 & Up & phenylethanolamine $\mathrm{N}$-methyltransferase \\
\hline A_23_P10172 & PRSS50 & 1.758832 & 0.00037 & 0.01872 & 5.708555 & $\mathrm{Up}$ & protease, serine 50 \\
\hline A_24_P142503 & SLC47A1 & 1.008499 & 0.000377 & 0.01889 & 5.69512 & $\mathrm{Up}$ & solute carrier family 47 member 1 \\
\hline A_33_P3289371 & PHACTR3 & 1.093945 & 0.000378 & 0.018958 & 5.690962 & Up & phosphatase and actin regulator 3 \\
\hline A_33_P3421118 & IL20RA & 1.257448 & 0.000386 & 0.019195 & 5.675376 & $\mathrm{Up}$ & interleukin 20 receptor subunit alpha \\
\hline A_23_P137573 & LEFTY2 & 1.262989 & 0.000389 & 0.019237 & 5.667495 & $\mathrm{Up}$ & left-right determination factor 2 \\
\hline A_23_P113777 & ITGBL1 & 1.325051 & 0.000395 & 0.019372 & 5.654497 & Up & integrin subunit beta like 1 \\
\hline A_33_P3274084 & RHBDL3 & 1.138528 & 0.000408 & 0.019769 & 5.628682 & $\mathrm{Up}$ & rhomboid like 3 \\
\hline A_23_P207194 & GH1 & 1.18446 & 0.000413 & 0.019911 & 5.618101 & $\mathrm{Up}$ & $\begin{array}{l}\text { growth hormone } 1 \\
\text { cadherin like and PC-esterase domain containing }\end{array}$ \\
\hline A_24_P943781 & CPED1 & 1.074957 & 0.000414 & 0.019933 & 5.616267 & Up & 1 \\
\hline A_33_P3307500 & STRA6 & 1.085733 & 0.000416 & 0.020011 & 5.612103 & Up & stimulated by retinoic acid 6 \\
\hline A_33_P3380642 & FRAS1 & 1.087367 & 0.000418 & 0.020041 & 5.608323 & $\mathrm{Up}$ & Fraser extracellular matrix complex subunit 1 \\
\hline A_33_P3394833 & FOXL2NB & 1.814458 & 0.000418 & 0.020041 & 5.608183 & Up & FOXL2 neighbor \\
\hline A_32_P780817 & CT45A1 & 1.206224 & 0.000429 & 0.020315 & 5.586514 & Up & cancer/testis antigen family 45 member A1 \\
\hline A_24_P51375 & \#N/A & 1.182894 & 0.000432 & 0.020315 & 5.58114 & $\mathrm{Up}$ & NA \\
\hline A_33_P3289121 & C2orf 40 & 1.024588 & 0.000433 & 0.020315 & 5.579578 & Up & chromosome 2 open reading frame 40 \\
\hline A_23_P500501 & FGFR3 & 1.024692 & 0.000434 & 0.020315 & 5.578659 & Up & fibroblast growth factor receptor 3 \\
\hline A_33_P3252281 & EYA4 & 1.461336 & 0.00044 & 0.02041 & 5.566737 & $\mathrm{Up}$ & EYA transcriptional coactivator and phosphatase \\
\hline
\end{tabular}




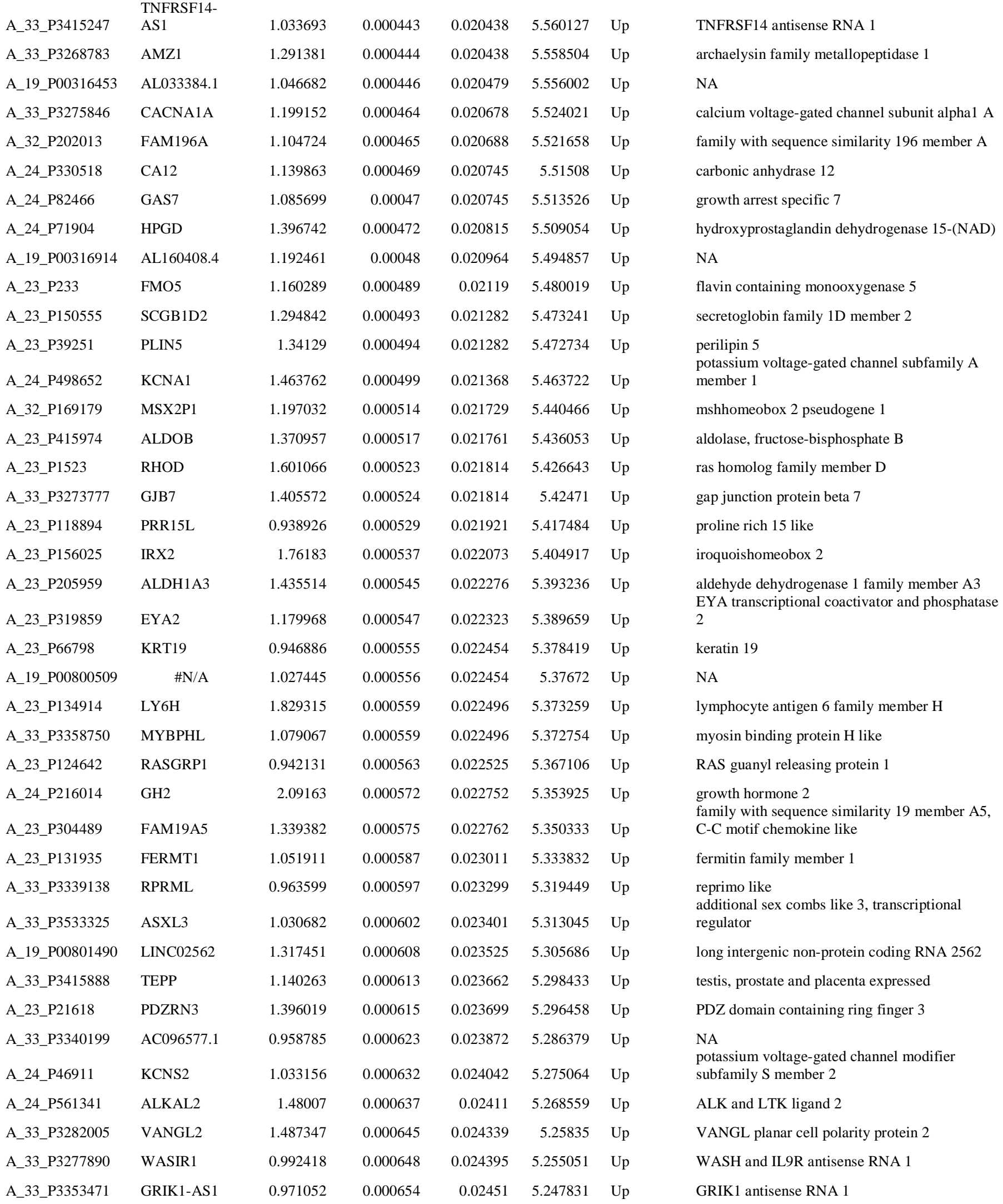




\begin{tabular}{|c|c|c|c|c|c|c|c|}
\hline A_32_P225816 & PRDM16 & 1.315384 & 0.000657 & 0.024583 & 5.244556 & $\mathrm{Up}$ & PR/SET domain 16 \\
\hline A_23_P148609 & PLAC1 & 0.962204 & 0.000658 & 0.024583 & 5.242831 & $\mathrm{Up}$ & placenta specific 1 \\
\hline A_33_P3271121 & SH3BP4 & 1.281724 & 0.00066 & 0.024583 & 5.240632 & $\mathrm{Up}$ & SH3 domain binding protein 4 \\
\hline A_33_P3285965 & NEUROD1 & 1.700584 & 0.000662 & 0.024605 & 5.238558 & $\mathrm{Up}$ & neuronal differentiation 1 \\
\hline A_33_P3288844 & IL6R & 1.168827 & 0.000663 & 0.02462 & 5.236673 & $\mathrm{Up}$ & interleukin 6 receptor \\
\hline A_33_P3298326 & FSHB & 2.112742 & 0.000664 & 0.02462 & 5.235787 & $\mathrm{Up}$ & follicle stimulating hormone beta subunit \\
\hline A_23_P207154 & $\mathrm{CSH} 2$ & 1.34608 & 0.000664 & 0.02462 & 5.235296 & $\mathrm{Up}$ & chorionic somatomammotropin hormone 2 \\
\hline A_33_P3321382 & CNTNAP3B & 1.057166 & 0.000677 & 0.024843 & 5.220864 & $\mathrm{Up}$ & contactin associated protein like 3B \\
\hline A_23_P159893 & CHRDL1 & 1.077118 & 0.000677 & 0.024843 & 5.220658 & Up & chordin like 1 \\
\hline A_24_P254506 & PAGE4 & 1.146619 & 0.000716 & 0.025673 & 5.176022 & Up & PAGE family member 4 \\
\hline A_23_P136777 & APOD & 1.229853 & 0.000717 & 0.025673 & 5.174762 & $\mathrm{Up}$ & apolipoprotein D \\
\hline A_23_P30983 & GJB7 & 1.662038 & 0.000739 & 0.026039 & 5.151262 & $\mathrm{Up}$ & $\begin{array}{l}\text { gap junction protein beta } 7 \\
\text { T-cell receptor beta variable 20/OR9-2 (non- }\end{array}$ \\
\hline A_33_P3273490 & TRBV20OR9-2 & 0.942031 & 0.000755 & 0.026226 & 5.134727 & Up & functional) \\
\hline A_23_P23292 & RXRG & 1.363857 & 0.000764 & 0.026479 & 5.125969 & $\mathrm{Up}$ & retinoid $\mathrm{X}$ receptor gamma \\
\hline A_23_P340263 & RNF175 & 1.370623 & 0.000772 & 0.026711 & 5.117906 & $\mathrm{Up}$ & ring finger protein 175 \\
\hline A_23_P398189 & IGSF11 & 1.162188 & 0.000776 & 0.026797 & 5.113508 & $\mathrm{Up}$ & immunoglobulin superfamily member 11 \\
\hline A_32_P187571 & $\mathrm{SCN} 2 \mathrm{~B}$ & 1.633253 & 0.000786 & 0.026893 & 5.103165 & $\mathrm{Up}$ & sodium voltage-gated channel beta subunit 2 \\
\hline A_23_P381746 & ASXL3 & 1.378008 & 0.00086 & 0.028195 & 5.033829 & Up & regulator \\
\hline A_23_P114713 & CYP4B1 & 1.134734 & 0.000878 & 0.02848 & 5.018238 & $\mathrm{Up}$ & cytochrome P450 family 4 subfamily B member 1 \\
\hline A_23_P392317 & DLGAP2 & 1.280103 & 0.000879 & 0.028513 & 5.016681 & $\mathrm{Up}$ & DLG associated protein 2 \\
\hline A_33_P3320197 & ALKAL2 & 1.220161 & 0.000889 & 0.028637 & 5.008349 & Up & ALK and LTK ligand 2 \\
\hline A_23_P110462 & PCDHB1 & 0.960895 & 0.000913 & 0.029158 & 4.987739 & $\mathrm{Up}$ & protocadherin beta 1 \\
\hline A_23_P150595 & TPH1 & 0.948973 & 0.000924 & 0.029301 & 4.978952 & $\mathrm{Up}$ & tryptophan hydroxylase 1 \\
\hline A_23_P154037 & AOX1 & 1.062065 & 0.000944 & 0.029642 & 4.96208 & Up & aldehyde oxidase 1 \\
\hline A_23_P86470 & $\mathrm{CH} 25 \mathrm{H}$ & 0.971876 & 0.000966 & 0.030028 & 4.944873 & $\mathrm{Up}$ & cholesterol 25-hydroxylase \\
\hline A_33_P3283824 & SLC39A8 & 1.133024 & 0.00098 & 0.03038 & 4.93375 & $\mathrm{Up}$ & solute carrier family 39 member 8 \\
\hline A_23_P143526 & S100B & 1.144173 & 0.000999 & 0.030653 & 4.91915 & $\mathrm{Up}$ & $\begin{array}{l}\text { S100 calcium binding protein B } \\
\text { MAM and LDL receptor class A domain }\end{array}$ \\
\hline A_33_P3292646 & MALRD1 & 1.208959 & 0.001004 & 0.0307 & 4.915254 & Up & containing 1 \\
\hline A_32_P34826 & B3GALT5-AS1 & 1.541162 & 0.001013 & 0.030874 & 4.908374 & $\mathrm{Up}$ & B3GALT5 antisense RNA 1 \\
\hline A_33_P3319022 & CLEC9A & 0.985478 & 0.001014 & 0.030874 & 4.907676 & Up & C-type lectin domain containing 9A \\
\hline A_24_P190472 & SLPI & 1.126312 & 0.001023 & 0.031057 & 4.900711 & $\mathrm{Up}$ & secretory leukocyte peptidase inhibitor \\
\hline A_32_P49199 & PGR & 1.356832 & 0.001051 & 0.031385 & 4.880628 & $\mathrm{Up}$ & progesterone receptor \\
\hline
\end{tabular}




\begin{tabular}{|c|c|c|c|c|c|c|c|}
\hline A_33_P3352467 & SSTR2 & 1.022938 & 0.001051 & 0.031385 & 4.880106 & Up & somatostatin receptor 2 \\
\hline A_19_P00321962 & AP000473.1 & 1.1851 & 0.001063 & 0.031601 & 4.871734 & Up & NA \\
\hline A_24_P236235 & FLRT2 & 0.96055 & 0.001102 & 0.032397 & 4.844468 & Up & fibronectinleucine rich transmembrane protein 2 \\
\hline A_33_P3232498 & C2orf71 & 0.973752 & 0.001115 & 0.032517 & 4.835307 & Up & chromosome 2 open reading frame 71 \\
\hline A_23_P1331 & COL13A1 & 1.167848 & 0.001139 & 0.032752 & 4.819329 & Up & $\begin{array}{l}\text { collagen type XIII alpha } 1 \text { chain } \\
\text { calcium voltage-gated channel auxiliary subunit }\end{array}$ \\
\hline A_33_P3373259 & CACNA2D3 & 1.461394 & 0.001151 & 0.03292 & 4.811744 & Up & alpha2delta 3 \\
\hline A_23_P73632 & NR0B1 & 1.455678 & 0.001157 & 0.03292 & 4.807777 & Up & nuclear receptor subfamily 0 group B member 1 \\
\hline A_23_P21092 & CALB2 & 1.452992 & 0.001175 & 0.033199 & 4.796121 & Up & calbindin 2 \\
\hline A_23_P146274 & STMN2 & 1.55252 & 0.001192 & 0.033466 & 4.785019 & Up & stathmin 2 \\
\hline A_23_P127781 & SCGB1D1 & 1.242168 & 0.001193 & 0.033466 & 4.784787 & Up & secretoglobin family $1 \mathrm{D}$ member 1 \\
\hline A_23_P213336 & FGF1 & 0.986971 & 0.001205 & 0.033585 & 4.777304 & Up & $\begin{array}{l}\text { fibroblast growth factor } 1 \\
\text { potassium voltage-gated channel subfamily A }\end{array}$ \\
\hline A_23_P48229 & KCNA1 & 1.237757 & 0.001218 & 0.03383 & 4.769327 & Up & member 1 \\
\hline A_23_P110052 & FOXL2 & 1.411075 & 0.001265 & 0.0345 & 4.740819 & Up & forkhead box L2 \\
\hline A_23_P308483 & MAP3K15 & 1.119098 & 0.001276 & 0.034639 & 4.734086 & Up & mitogen-activated protein kinase kinasekinase 15 \\
\hline A_33_P3304082 & AC100872.1 & 1.03319 & 0.001277 & 0.034639 & 4.733848 & Up & NA \\
\hline A_33_P3227269 & GATA3-AS1 & 1.53053 & 0.001296 & 0.034887 & 4.722827 & Up & GATA3 antisense RNA 1 \\
\hline A_24_P326491 & MKX & 1.009479 & 0.001298 & 0.034889 & 4.721501 & Up & mohawkhomeobox \\
\hline A_23_P434398 & TXLNB & 1.116641 & 0.001346 & 0.035471 & 4.694487 & Up & taxilin beta \\
\hline A_33_P3709150 & $\mathrm{T}$ & 0.957229 & 0.001354 & 0.035473 & 4.690142 & Up & $\mathrm{T}$ brachyury transcription factor \\
\hline A_33_P3527317 & LOC148638 & 1.189409 & 0.001354 & 0.035473 & 4.689917 & Up & uncharacterized LOC148638 \\
\hline A_32_P183765 & ERBB4 & 1.80022 & 0.001409 & 0.036262 & 4.66067 & Up & erb-b2 receptor tyrosine kinase 4 \\
\hline A_19_P00811193 & \#N/A & 0.967772 & 0.00141 & 0.036262 & 4.660162 & Up & $\begin{array}{l}\text { NA } \\
\text { protein phosphatase } 1 \text { regulatory inhibitor subunit }\end{array}$ \\
\hline A_23_P129835 & PPP1R1B & 1.133206 & 0.001419 & 0.036362 & 4.655157 & Up & $1 \mathrm{~B}$ \\
\hline A_23_P79968 & PCSK2 & 1.403328 & 0.001437 & 0.036661 & 4.645726 & Up & proproteinconvertasesubtilisin/kexin type 2 \\
\hline A_33_P3405459 & FNDC11 & 1.20722 & 0.001446 & 0.036818 & 4.641233 & Up & fibronectin type III domain containing 11 \\
\hline A_23_P320261 & DMKN & 0.95874 & 0.001475 & 0.037025 & 4.626565 & Up & dermokine \\
\hline A_23_P200780 & TGFBR3 & 1.016642 & 0.00148 & 0.037043 & 4.623865 & Up & transforming growth factor beta receptor 3 \\
\hline A_23_P6381 & MN1 & 0.940638 & 0.001497 & 0.037402 & 4.615481 & Up & MN1 proto-oncogene, transcriptional regulator \\
\hline A_33_P3411035 & SLIT1 & 1.425281 & 0.001504 & 0.037429 & 4.612055 & Up & $\begin{array}{l}\text { slit guidance ligand } 1 \\
\text { potassium voltage-gated channel modifier }\end{array}$ \\
\hline A_23_P210581 & KCNG1 & 0.947554 & 0.001562 & 0.038407 & 4.584504 & Up & subfamily G member 1 \\
\hline A_23_P54340 & MEGF11 & 1.058389 & 0.001582 & 0.038509 & 4.575181 & Up & multiple EGF like domains 11 \\
\hline A_23_P73571 & MUM1L1 & 1.588137 & 0.001589 & 0.038563 & 4.571829 & Up & MUM1 like 1 \\
\hline A_33_P3846653 & KRT19P2 & 1.050094 & 0.001666 & 0.039613 & 4.537271 & Up & keratin 19 pseudogene 2 \\
\hline A_23_P11685 & PLA2G4A & 0.933455 & 0.001677 & 0.039754 & 4.532537 & Up & phospholipase A2 group IVA \\
\hline A_23_P139687 & ERP27 & 0.966953 & 0.001688 & 0.039785 & 4.52741 & Up & endoplasmic reticulum protein 27 \\
\hline A_33_P3351279 & GPR37L1 & 0.966182 & 0.001697 & 0.039868 & 4.523641 & Up & G protein-coupled receptor 37 like 1 \\
\hline A_33_P3311503 & SMR3B & 0.956584 & 0.001702 & 0.039925 & 4.521576 & Up & submaxillary gland androgen regulated protein $3 \mathrm{~B}$ \\
\hline A_33_P3336686 & CLIC3 & 0.9489 & 0.001714 & 0.04004 & 4.51625 & Up & chloride intracellular channel 3 \\
\hline A_23_P421306 & SYT12 & 1.356431 & 0.001721 & 0.040069 & 4.513554 & Up & synaptotagmin 12 \\
\hline A_33_P3219939 & CUBN & 0.988697 & 0.00175 & 0.040474 & 4.501436 & Up & cubilin \\
\hline A_32_P34046 & HFM1 & 1.161494 & 0.001751 & 0.040474 & 4.500666 & Up & HFM1, ATP dependent DNA helicase homolog \\
\hline A_33_P3459365 & LINC00880 & 1.060831 & 0.001756 & 0.040509 & 4.498854 & Up & long intergenic non-protein coding RNA 880 \\
\hline
\end{tabular}




\begin{tabular}{|c|c|c|c|c|c|c|c|}
\hline A_23_P377094 & PNMA3 & 1.024371 & 0.001846 & 0.041758 & 4.462341 & Up & paraneoplastic Ma antigen 3 \\
\hline A_33_P3343182 & IQCA1 & 1.174187 & 0.00185 & 0.041796 & 4.461004 & Up & IQ motif containing with AAA domain 1 \\
\hline A_33_P3729375 & \#N/A & 1.126453 & 0.001854 & 0.041826 & 4.459231 & Up & NA \\
\hline A_33_P3387766 & LINC00908 & 1.113277 & 0.00189 & 0.042112 & 4.445516 & Up & $\begin{array}{l}\text { long intergenic non-protein coding RNA } 908 \\
\text { cadherin like and PC-esterase domain containing }\end{array}$ \\
\hline A_24_P187799 & CPED1 & 0.969089 & 0.001898 & 0.042255 & 4.442173 & Up & 1 \\
\hline A_23_P69154 & FAM198A & 1.114636 & 0.001939 & 0.042586 & 4.426766 & Up & family with sequence similarity 198 member A \\
\hline A_33_P3214988 & EFCAB1 & 1.067562 & 0.00196 & 0.042762 & 4.419076 & Up & EF-hand calcium binding domain 1 \\
\hline A_23_P344568 & FAM124A & 1.051178 & 0.002042 & 0.043747 & 4.389351 & Up & family with sequence similarity 124 member A \\
\hline A_23_P117851 & CPLX3 & 1.042413 & 0.002111 & 0.044804 & 4.365519 & Up & complexin 3 \\
\hline A_19_P00810522 & \#N/A & 1.16815 & 0.002131 & 0.044805 & 4.358693 & Up & NA \\
\hline A_23_P10232 & BANK1 & 1.035706 & 0.002181 & 0.045387 & 4.342172 & Up & B-cell scaffold protein with ankyrin repeats 1 \\
\hline A_23_P338534 & HIF3A & 1.003302 & 0.002203 & 0.045626 & 4.335127 & Up & hypoxia inducible factor 3 alpha subunit \\
\hline A_23_P146294 & EFCAB1 & 1.496561 & 0.00224 & 0.045987 & 4.323259 & Up & EF-hand calcium binding domain 1 \\
\hline A_24_P929322 & FLJ42393 & 1.329889 & 0.00225 & 0.046061 & 4.319918 & Up & uncharacterized LOC401105 \\
\hline A_24_P418203 & CNTNAP3 & 1.053068 & 0.002253 & 0.046061 & 4.319016 & Up & contactin associated protein-like 3 \\
\hline A_23_P7684 & CCNJL & 1.325275 & 0.002253 & 0.046061 & 4.319 & Up & cyclin J like \\
\hline A_33_P3412428 & ADAM32 & 0.990478 & 0.002281 & 0.046295 & 4.310264 & Up & ADAM metallopeptidase domain 32 \\
\hline A_33_P3215729 & RPS3AP14 & 1.011099 & 0.002429 & 0.047738 & 4.265334 & Up & ribosomal protein S3a pseudogene 14 \\
\hline A_23_P64121 & KIAA1549L & 1.381953 & 0.00253 & 0.048635 & 4.236572 & Up & KIAA1549 like \\
\hline A_23_P80242 & SEZ6L & 1.445032 & 0.002533 & 0.048676 & 4.235657 & Up & seizure related 6 homolog like \\
\hline A_33_P3302453 & LINC00242 & 1.002045 & 0.002592 & 0.049393 & 4.219266 & Up & long intergenic non-protein coding RNA 242 \\
\hline A_24_P33446 & KRTAP5-AS1 & -1.18091 & 7.62E-08 & $9.03 \mathrm{E}-05$ & -17.1132 & Down & KRTAP5-1/KRTAP5-2 antisense RNA 1 \\
\hline A_33_P3259028 & TRIM24 & -0.41581 & $1.15 \mathrm{E}-06$ & 0.000557 & -12.2547 & Down & tripartite motif containing 24 \\
\hline A_33_P3212684 & \#N/A & -0.76271 & $1.64 \mathrm{E}-06$ & 0.000718 & -11.7307 & Down & $\begin{array}{l}\text { NA } \\
\text { olfactory receptor family } 4 \text { subfamily A member }\end{array}$ \\
\hline A_23_P139099 & OR4A15 & -0.42416 & 2.37E-06 & 0.000899 & -11.2005 & Down & 15 \\
\hline A_23_P159974 & KLHL13 & -0.37716 & $3.9 \mathrm{E}-06$ & 0.00122 & -10.5164 & Down & kelch like family member 13 \\
\hline A_33_P3269503 & KRT87P & -0.94844 & 4.21E-06 & 0.001298 & -10.4139 & Down & keratin 87 pseudogene \\
\hline A_19_P00802970 & ZRANB2-AS1 & -0.56876 & 4.36E-06 & 0.001326 & -10.3675 & Down & ZRANB2 antisense RNA 1 \\
\hline A_19_P00323612 & \#N/A & -0.41168 & 4.64E-06 & 0.001358 & -10.2869 & Down & NA \\
\hline A_19_P00326199 & SINHCAF & -0.52736 & 4.72E-06 & 0.001358 & -10.2635 & Down & SIN3-HDAC complex associated factor \\
\hline A_23_P254831 & MAGEB2 & -0.36511 & $5.16 \mathrm{E}-06$ & 0.001443 & -10.1497 & Down & MAGE family member B2 \\
\hline
\end{tabular}




\begin{tabular}{|c|c|c|c|c|c|c|c|}
\hline A_19_P00327423 & \#N/A & -0.74048 & $5.89 \mathrm{E}-06$ & 0.001597 & -9.97785 & Down & NA \\
\hline A_19_P00800667 & LINC00398 & -0.31923 & $6.4 \mathrm{E}-06$ & 0.00171 & -9.87313 & Down & long intergenic non-protein coding RNA 398 \\
\hline A_19_P00811704 & \#N/A & -0.49672 & $6.45 \mathrm{E}-06$ & 0.00171 & -9.86335 & Down & NA \\
\hline A_19_P00315872 & \#N/A & -0.56281 & $1.14 \mathrm{E}-05$ & 0.0026 & -9.16497 & Down & NA \\
\hline A_23_P121596 & PPBP & -1.54696 & $1.17 \mathrm{E}-05$ & 0.002615 & -9.13962 & Down & pro-platelet basic protein \\
\hline A_23_P328145 & FAM71D & -0.93751 & $1.24 \mathrm{E}-05$ & 0.002678 & -9.06826 & Down & family with sequence similarity 71 member $D$ \\
\hline A_19_P00326831 & \#N/A & -0.74662 & $1.25 \mathrm{E}-05$ & 0.002678 & -9.06195 & Down & NA \\
\hline A_23_P382682 & NOXRED1 & -0.73831 & $1.4 \mathrm{E}-05$ & 0.002852 & -8.92458 & Down & $\begin{array}{l}\text { NADP dependent oxidoreductase domain } \\
\text { containing } 1\end{array}$ \\
\hline A_33_P3235048 & TRIM77 & -0.40971 & $1.46 \mathrm{E}-05$ & 0.002909 & -8.88124 & Down & tripartite motif containing 77 \\
\hline A_19_P00323829 & \#N/A & -0.90037 & $2.08 \mathrm{E}-05$ & 0.003738 & -8.47651 & Down & NA \\
\hline A_19_P00330864 & \#N/A & -1.06535 & $2.11 \mathrm{E}-05$ & 0.003766 & -8.46369 & Down & NA \\
\hline A_19_P00801104 & \#N/A & -0.54282 & $2.66 \mathrm{E}-05$ & 0.004416 & -8.20936 & Down & NA \\
\hline A_23_P212284 & POC1A & -0.3445 & $2.71 \mathrm{E}-05$ & 0.004457 & -8.18814 & Down & POC1 centriolar protein A \\
\hline A_19_P00813027 & \#N/A & -0.38569 & $2.75 \mathrm{E}-05$ & 0.004497 & -8.17297 & Down & NA \\
\hline A_33_P3325502 & ARHGAP29 & -0.93195 & $2.78 \mathrm{E}-05$ & 0.004538 & -8.15889 & Down & Rho GTPase activating protein 29 \\
\hline A_23_P315815 & NRG1 & -0.34435 & 2.93E-05 & 0.004679 & -8.10578 & Down & neuregulin 1 \\
\hline A_33_P3335825 & PLCD4 & -0.74316 & $3.14 \mathrm{E}-05$ & 0.004861 & -8.02939 & Down & phospholipase $\mathrm{C}$ delta 4 \\
\hline A_23_P57588 & GTSE1 & -0.80101 & 4.28E-05 & 0.005822 & -7.70706 & Down & G2 and S-phase expressed 1 \\
\hline A_23_P366983 & TRHDE & -0.85404 & $4.36 \mathrm{E}-05$ & 0.005857 & -7.6877 & Down & thyrotropin releasing hormone degrading enzyme \\
\hline A_33_P3405680 & ARHGEF3-AS1 & -0.50887 & 4.6E-05 & 0.006063 & -7.63133 & Down & ARHGEF3 antisense RNA 1 \\
\hline A_24_P911676 & SOX4 & -0.48583 & 4.82E-05 & 0.006272 & -7.58518 & Down & SRY-box 4 \\
\hline A_19_P00807368 & \#N/A & -0.29663 & $5.04 \mathrm{E}-05$ & 0.006496 & -7.53945 & Down & $\begin{array}{l}\text { NA } \\
\text { deleted in lymphocytic leukemia } 1 \text { (non-protein }\end{array}$ \\
\hline A_19_P00320026 & DLEU1 & -0.42962 & $5.06 \mathrm{E}-05$ & 0.006498 & -7.53609 & Down & coding) \\
\hline A_19_P00325235 & \#N/A & -0.6208 & $5.14 \mathrm{E}-05$ & 0.00653 & -7.51885 & Down & NA \\
\hline A_19_P00321005 & \#N/A & -0.9916 & $5.6 \mathrm{E}-05$ & 0.006971 & -7.43242 & Down & NA \\
\hline A_23_P118174 & PLK1 & -0.46167 & $5.73 \mathrm{E}-05$ & 0.007069 & -7.40971 & Down & polo like kinase 1 \\
\hline A_33_P3271945 & HAUS6 & -0.41613 & $5.81 \mathrm{E}-05$ & 0.007101 & -7.3965 & Down & HAUS augmin like complex subunit 6 \\
\hline A_19_P00324653 & GPALPP1 & -0.33064 & $6.19 \mathrm{E}-05$ & 0.007399 & -7.33303 & Down & GPALPP motifs containing 1 \\
\hline A_23_P124417 & BUB1 & -1.18787 & $6.27 \mathrm{E}-05$ & 0.007429 & -7.32071 & Down & BUB1 mitotic checkpoint serine/threonine kinase \\
\hline A_24_P145629 & SERINC2 & -0.77141 & $6.37 \mathrm{E}-05$ & 0.007486 & -7.30502 & Down & serine incorporator 2 \\
\hline A_33_P3230548 & KIF14 & -1.33322 & $6.4 \mathrm{E}-05$ & 0.007486 & -7.29949 & Down & kinesin family member 14 \\
\hline
\end{tabular}




\begin{tabular}{|c|c|c|c|c|c|c|c|}
\hline A_33_P3598466 & HSP90AB6P & -0.61836 & $6.54 \mathrm{E}-05$ & 0.007624 & -7.27875 & Down & $\begin{array}{l}\text { heat shock protein } 90 \text { alpha family class B } \\
\text { member } 6 \text {, pseudogene }\end{array}$ \\
\hline A_33_P3421973 & FRMPD3 & -0.97088 & $6.84 \mathrm{E}-05$ & 0.007761 & -7.23466 & Down & FERM and PDZ domain containing 3 \\
\hline A_23_P18579 & PTTG2 & -0.3236 & $7.36 \mathrm{E}-05$ & 0.008159 & -7.16262 & Down & $\begin{array}{l}\text { pituitary tumor-transforming } 2 \\
\text { RAN, member RAS oncogene family pseudogene }\end{array}$ \\
\hline A_19_P00329691 & RANP3 & -0.44835 & 7.63E-05 & 0.008328 & -7.12757 & Down & 3 \\
\hline A_24_P219114 & SEL1L & -0.34542 & $8.34 \mathrm{E}-05$ & 0.008626 & -7.04271 & Down & SEL1L ERAD E3 ligase adaptor subunit \\
\hline A_33_P3350488 & NUSAP1 & -0.45817 & $8.45 \mathrm{E}-05$ & 0.008651 & -7.02997 & Down & nucleolar and spindle associated protein 1 \\
\hline A_33_P3343720 & CELA3A & -0.49673 & $8.54 \mathrm{E}-05$ & 0.008656 & -7.01888 & Down & chymotrypsin like elastase family member $3 \mathrm{~A}$ \\
\hline A_19_P00331977 & \#N/A & -0.4191 & $8.65 \mathrm{E}-05$ & 0.008717 & -7.00758 & Down & NA \\
\hline A_19_P00331084 & \#N/A & -0.48869 & 9.37E-05 & 0.009105 & -6.93101 & Down & NA \\
\hline A_19_P00322169 & \#N/A & -0.61999 & 0.000103 & 0.009726 & -6.84557 & Down & NA \\
\hline A_33_P3263002 & TMEM262 & -0.63529 & 0.000104 & 0.009737 & -6.83519 & Down & transmembrane protein 262 \\
\hline A_33_P3284345 & NRG1 & -0.32711 & 0.000106 & 0.009862 & -6.81496 & Down & neuregulin 1 \\
\hline A_23_P81926 & PSORS1C2 & -0.36148 & 0.000107 & 0.009869 & -6.80951 & Down & psoriasis susceptibility 1 candidate 2 \\
\hline A_19_P00332459 & DHX15 & -1.24559 & 0.000108 & 0.009961 & -6.79737 & Down & DEAH-box helicase 15 \\
\hline A_23_P35219 & NEK2 & -1.05848 & 0.000109 & 0.010008 & -6.78932 & Down & NIMA related kinase 2 \\
\hline A_33_P3260511 & $\mathrm{CICP} 24$ & -0.35944 & 0.000109 & 0.010009 & -6.78677 & Down & capicua transcriptional repressor pseudogene 24 \\
\hline A_33_P3403429 & LRRC69 & -0.4054 & 0.000112 & 0.010141 & -6.76056 & Down & leucine rich repeat containing 69 \\
\hline A_33_P3354067 & AC092821.2 & -0.31328 & 0.000124 & 0.010658 & -6.66572 & Down & NA \\
\hline A_19_P00801050 & \#N/A & -0.48588 & 0.000125 & 0.010685 & -6.65847 & Down & NA \\
\hline A_23_P70007 & HMMR & -0.49422 & 0.00013 & 0.010887 & -6.62632 & Down & hyaluronan mediated motility receptor \\
\hline A_33_P3770642 & LINC00929 & -0.84694 & 0.00013 & 0.010887 & -6.62288 & Down & long intergenic non-protein coding RNA 929 \\
\hline A_19_P00317247 & PTP4A1 & -0.3095 & 0.000135 & 0.011079 & -6.58899 & Down & protein tyrosine phosphatase type IVA, member 1 \\
\hline A_19_P00328255 & \#N/A & -0.29491 & 0.000136 & 0.011131 & -6.58118 & Down & NA \\
\hline A_23_P19369 & CARMIL1 & -0.377 & 0.000143 & 0.011485 & -6.53694 & Down & capping protein regulator and myosin 1 linker 1 \\
\hline A_33_P3322125 & DAZAP2 & -0.33412 & 0.000145 & 0.011604 & -6.52583 & Down & DAZ associated protein 2 \\
\hline A_33_P3333177 & \#N/A & -0.62324 & 0.000146 & 0.011665 & -6.51937 & Down & NA \\
\hline A_23_P104651 & CDCA5 & -0.36513 & 0.000146 & 0.011689 & -6.51579 & Down & cell division cycle associated 5 \\
\hline A_19_P00328658 & \#N/A & -0.32397 & 0.000148 & 0.011752 & -6.5092 & Down & NA \\
\hline A_19_P00319194 & \#N/A & -0.87023 & 0.000152 & 0.01191 & -6.48015 & Down & NA \\
\hline A_19_P00330750 & \#N/A & -0.48391 & 0.000153 & 0.01191 & -6.47897 & Down & NA \\
\hline A_19_P00800106 & \#N/A & -0.51725 & 0.000155 & 0.012049 & -6.46641 & Down & NA \\
\hline A_19_P00800448 & \#N/A & -0.46945 & 0.000156 & 0.012123 & -6.45755 & Down & NA \\
\hline
\end{tabular}




\begin{tabular}{|c|c|c|c|c|c|c|c|}
\hline A_33_P3209703 & \#N/A & -0.38403 & 0.000159 & 0.012272 & -6.44165 & Down & NA \\
\hline A_19_P00801014 & ANTXR2 & -0.33022 & 0.000166 & 0.01256 & -6.40275 & Down & anthrax toxin receptor 2 \\
\hline A_19_P00327255 & \#N/A & -0.33261 & 0.000168 & 0.012611 & -6.39321 & Down & NA \\
\hline A_19_P00321594 & FBXL5 & -0.89313 & 0.00017 & 0.012633 & -6.38353 & Down & F-box and leucine rich repeat protein 5 \\
\hline A_33_P3420704 & \#N/A & -0.3589 & 0.00017 & 0.012666 & -6.37957 & Down & NA \\
\hline A_33_P3316903 & RBPMS-AS1 & -0.40474 & 0.000175 & 0.012856 & -6.35387 & Down & RBPMS antisense RNA 1 \\
\hline A_23_P300100 & PLA2G2D & -0.52315 & 0.000179 & 0.012985 & -6.33706 & Down & phospholipase A2 group IID \\
\hline A_33_P3295077 & AC092162.1 & -0.32979 & 0.000182 & 0.013153 & -6.31903 & Down & NA \\
\hline A_33_P3375334 & DAB1 & -1.09252 & 0.000186 & 0.013284 & -6.30295 & Down & $\begin{array}{l}\text { DAB1, reelin adaptor protein } \\
\text { testis-specific transcript, Y-linked } 13 \text { (non-protein }\end{array}$ \\
\hline A_23_P11397 & TTTY13 & -0.69508 & 0.000186 & 0.013284 & -6.3019 & Down & \\
\hline A_23_P346884 & RBPJL & -0.32051 & 0.000195 & 0.013736 & -6.25896 & Down & immunoglobulin kappa $\mathrm{J}$ region like \\
\hline A_19_P00804053 & \#N/A & -0.35416 & 0.000197 & 0.013821 & -6.24831 & Down & NA \\
\hline A_19_P00322074 & \#N/A & -0.36755 & 0.0002 & 0.013821 & -6.23712 & Down & NA \\
\hline A_19_P00327072 & \#N/A & -0.34689 & 0.000201 & 0.013821 & -6.23408 & Down & NA \\
\hline A_33_P3386344 & FANCA & -0.32132 & 0.000204 & 0.01395 & -6.22023 & Down & Fanconianemia complementation group A \\
\hline A_33_P3340468 & CENPI & -0.73725 & 0.000205 & 0.014011 & -6.21277 & Down & centromere protein I \\
\hline A_19_P00330152 & \#N/A & -0.44423 & 0.000206 & 0.014017 & -6.21017 & Down & NA \\
\hline A_19_P00800639 & \#N/A & -0.48611 & 0.000207 & 0.014064 & -6.20523 & Down & NA \\
\hline A_19_P00331152 & \#N/A & -0.61926 & 0.00021 & 0.014139 & -6.19498 & Down & NA \\
\hline A_19_P00811393 & \#N/A & -0.30534 & 0.000215 & 0.014373 & -6.17502 & Down & NA \\
\hline A_33_P3304268 & LOC643327 & -0.67616 & 0.000216 & 0.014405 & -6.16802 & Down & uncharacterized LOC643327 \\
\hline A_33_P3214690 & NLGN1 & -0.39399 & 0.000219 & 0.014495 & -6.15644 & Down & neuroligin 1 \\
\hline A_33_P3290729 & POU3F2 & -1.77448 & 0.000219 & 0.014495 & -6.15601 & Down & POU class 3 homeobox 2 \\
\hline A_23_P425332 & PPP4R4 & -0.37974 & 0.000219 & 0.014495 & -6.15533 & Down & protein phosphatase 4 regulatory subunit 4 \\
\hline A_33_P3345469 & GPR6 & -0.42562 & 0.000221 & 0.014537 & -6.148 & Down & G protein-coupled receptor 6 \\
\hline A_23_P414211 & PAX4 & -0.32483 & 0.000223 & 0.014561 & -6.14213 & Down & paired box 4 \\
\hline A_33_P3413224 & AC092755.2 & -0.39132 & 0.000226 & 0.014718 & -6.12784 & Down & NA \\
\hline A_19_P00316773 & \#N/A & -0.95664 & 0.000227 & 0.014718 & -6.12671 & Down & $\begin{array}{l}\text { NA } \\
\text { additional sex combs like } 2 \text {, transcriptional }\end{array}$ \\
\hline A_33_P3248610 & ASXL2 & -0.31774 & 0.000227 & 0.014718 & -6.12588 & Down & regulator \\
\hline A_19_P00320549 & \#N/A & -0.35255 & 0.000231 & 0.014898 & -6.11129 & Down & NA \\
\hline A_33_P3243454 & IGFL3 & -1.0725 & 0.000233 & 0.014996 & -6.10166 & Down & IGF like family member 3 \\
\hline A_33_P3416414 & PSAT1P1 & -0.62563 & 0.000236 & 0.015056 & -6.09036 & Down & $\begin{array}{l}\text { phosphoserine aminotransferase } 1 \text { pseudogene } 1 \\
\text { glutamate ionotropic receptor kainate type subunit }\end{array}$ \\
\hline A_33_P3307144 & GRIK2 & -0.39339 & 0.000237 & 0.015075 & -6.08797 & Down & 2 \\
\hline A_23_P60016 & PTTG3P & -0.61312 & 0.00024 & 0.015106 & -6.07843 & Down & pituitary tumor-transforming 3 , pseudogene \\
\hline A_33_P3230254 & NCAPG & -0.80641 & 0.000243 & 0.015185 & -6.06626 & Down & non-SMC condensin I complex subunit G \\
\hline A_33_P3265549 & SLC19A1 & -0.38232 & 0.000244 & 0.015241 & -6.0618 & Down & solute carrier family 19 member 1 \\
\hline A_33_P3629151 & \#N/A & -0.30303 & 0.000252 & 0.015491 & -6.03643 & Down & NA \\
\hline A_32_P64598 & LINC02156 & -0.97878 & 0.000253 & 0.015555 & -6.03164 & Down & long intergenic non-protein coding RNA 2156 \\
\hline A_23_P166993 & EPHB1 & -0.48094 & 0.000255 & 0.015611 & -6.02497 & Down & EPH receptor B1 \\
\hline A_24_P141707 & INHBE & -0.87769 & 0.000258 & 0.015701 & -6.01625 & Down & inhibin beta $\mathrm{E}$ subunit \\
\hline A_19_P00324607 & XIST_EXON1 & -0.52726 & 0.000258 & 0.015701 & -6.01367 & Down & $\mathrm{X}$-chromosome inactivation gene exon 1 \\
\hline A_23_P112187 & FIBCD1 & -0.32728 & 0.000259 & 0.015703 & -6.01152 & Down & fibrinogen $\mathrm{C}$ domain containing 1 \\
\hline
\end{tabular}




\begin{tabular}{|c|c|c|c|c|c|c|c|}
\hline A_19_P00330161 & RGS5 & -0.31195 & 0.000261 & 0.015752 & -6.00355 & Down & regulator of G protein signaling 5 \\
\hline A_19_P00810938 & \#N/A & -0.47038 & 0.000264 & 0.015866 & -5.99368 & Down & NA \\
\hline A_33_P3671729 & FLJ44715 & -0.66365 & 0.000266 & 0.015901 & -5.98817 & Down & uncharacterized LOC386671 \\
\hline A_23_P63032 & GUCA2B & -0.45895 & 0.000269 & 0.016011 & -5.97986 & Down & guanylatecyclase activator 2B \\
\hline A_23_P65757 & CCNB2 & -0.68899 & 0.000272 & 0.016127 & -5.97008 & Down & cyclin B2 \\
\hline A_33_P3221761 & КМT2B & -0.33926 & 0.000273 & 0.016143 & -5.96538 & Down & lysine methyltransferase $2 \mathrm{~B}$ \\
\hline A_23_P342138 & ADAMTSL1 & -0.30204 & 0.000274 & 0.016143 & -5.96357 & Down & ADAMTS like 1 \\
\hline $\begin{array}{l}\text { A_19_P00329997 } \\
\text { ERCC- }\end{array}$ & \#N/A & -0.77956 & 0.000275 & 0.016166 & -5.95971 & Down & NA \\
\hline 00126_533 & \#N/A & -0.61974 & 0.00028 & 0.016335 & -5.94611 & Down & NA \\
\hline A_19_P00807001 & \#N/A & -0.59089 & 0.000281 & 0.016399 & -5.9404 & Down & NA \\
\hline ERCC-00014_76 & \#N/A & -0.58213 & 0.000289 & 0.016663 & -5.9164 & Down & NA \\
\hline A_19_P00330883 & \#N/A & -0.44021 & 0.000294 & 0.016851 & -5.90168 & Down & NA \\
\hline A_19_P00806769 & LINC01122 & -0.33078 & 0.000297 & 0.016851 & -5.8936 & Down & long intergenic non-protein coding RNA 1122 \\
\hline A_33_P3300152 & \#N/A & -0.3139 & 0.000297 & 0.016851 & -5.89333 & Down & NA \\
\hline A_33_P3255001 & LINC00691 & -0.67432 & 0.000299 & 0.016851 & -5.88871 & Down & long intergenic non-protein coding RNA 691 \\
\hline A_32_P204376 & ANKRD20A2 & -0.82794 & 0.0003 & 0.016851 & -5.88524 & Down & ankyrin repeat domain 20 family member $\mathrm{A} 2$ \\
\hline A_19_P00325303 & \#N/A & -0.29931 & 0.000302 & 0.016931 & -5.88008 & Down & NA \\
\hline A_24_P43810 & FAM83A & -1.14146 & 0.000307 & 0.017077 & -5.86726 & Down & family with sequence similarity 83 member A \\
\hline A_33_P3296281 & \#N/A & -1.00736 & 0.000308 & 0.017112 & -5.86396 & Down & NA \\
\hline A_33_P3395733 & AL049795.1 & -0.52782 & 0.000309 & 0.017112 & -5.86154 & Down & NA \\
\hline A_33_P3229552 & LRFN1 & -0.3534 & 0.000309 & 0.017112 & -5.86091 & Down & containing 1 \\
\hline A_19_P00811105 & \#N/A & -0.35467 & 0.000309 & 0.017112 & -5.86002 & Down & NA \\
\hline A_23_P201022 & PKLR & -0.29705 & 0.000315 & 0.017267 & -5.84461 & Down & pyruvate kinase, liver and $\mathrm{RBC}$ \\
\hline A_33_P3840512 & SLC25A15 & -0.30272 & 0.000317 & 0.017267 & -5.83945 & Down & solute carrier family 25 member 15 \\
\hline A_33_P3389758 & \#N/A & -0.38846 & 0.000317 & 0.017267 & -5.83831 & Down & $\begin{array}{l}\text { NA } \\
\text { gamma-aminobutyric acid type A receptor theta }\end{array}$ \\
\hline A_24_P367410 & GABRQ & -0.68191 & 0.000319 & 0.017276 & -5.83492 & Down & subunit \\
\hline A_19_P00324404 & \#N/A & -0.32077 & 0.000322 & 0.017376 & -5.82646 & Down & NA \\
\hline A_23_P206059 & PRC1 & -0.3017 & 0.000324 & 0.01742 & -5.82114 & Down & protein regulator of cytokinesis 1 \\
\hline A_23_P14083 & AMIGO2 & -0.44866 & 0.000326 & 0.017507 & -5.81479 & Down & adhesion molecule with Ig like domain 2 \\
\hline A_24_P587443 & AC025278.1 & -0.61913 & 0.000329 & 0.017606 & -5.80688 & Down & NA \\
\hline A_33_P3248028 & \#N/A & -0.68935 & 0.000333 & 0.017746 & -5.79811 & Down & NA \\
\hline A_33_P3274001 & SUMO2P8 & -0.31129 & 0.000336 & 0.017778 & -5.79043 & Down & SUMO2 pseudogene 8 \\
\hline A_23_P57379 & $\mathrm{CDC} 45$ & -0.42634 & 0.000337 & 0.017778 & -5.78764 & Down & cell division cycle 45 \\
\hline A_23_P254733 & CENPU & -0.53283 & 0.00034 & 0.017833 & -5.78005 & Down & centromere protein $\mathrm{U}$ \\
\hline A_24_P9833 & PRDM11 & -0.38799 & 0.000341 & 0.017833 & -5.77904 & Down & PR/SET domain 11 \\
\hline A_33_P3311543 & \#N/A & -0.42903 & 0.000344 & 0.017933 & -5.7716 & Down & $\begin{array}{l}\text { NA } \\
\text { methylenetetrahydrofolate dehydrogenase }\end{array}$ \\
\hline A_33_P3216272 & LOC100133920 & -0.29115 & 0.000348 & 0.01814 & -5.75995 & Down & (NADP+ dependent) 1-like pseudogene \\
\hline A_19_P00802008 & \#N/A & -0.36753 & 0.000352 & 0.018266 & -5.75094 & Down & NA \\
\hline A_19_P00806281 & \#N/A & -0.45576 & 0.000355 & 0.018351 & -5.74313 & Down & NA \\
\hline A_19_P00800105 & \#N/A & -0.48555 & 0.000358 & 0.018376 & -5.73826 & Down & $\begin{array}{l}\text { NA } \\
\text { cytochrome P450 family } 27 \text { subfamily B member }\end{array}$ \\
\hline A_23_P36397 & CYP27B1 & -0.41362 & 0.00037 & 0.018715 & -5.70973 & Down & 1 \\
\hline
\end{tabular}




\begin{tabular}{|c|c|c|c|c|c|c|c|}
\hline A_24_P227091 & KIF11 & -0.32887 & 0.000374 & 0.018869 & -5.70094 & Down & kinesin family member 11 \\
\hline A_33_P3413518 & $\begin{array}{c}\text { \#N/A } \\
\text { LL22NC03- }\end{array}$ & -0.44929 & 0.000375 & 0.018883 & -5.69938 & Down & NA \\
\hline A_33_P3337124 & $63 \mathrm{E} 9.3$ & -0.34339 & 0.000376 & 0.01889 & -5.69708 & Down & uncharacterized LOC648691 \\
\hline A_19_P00811963 & \#N/A & -0.4231 & 0.000381 & 0.019036 & -5.68577 & Down & NA \\
\hline A_19_P00801430 & \#N/A & -0.33418 & 0.000382 & 0.019068 & -5.68342 & Down & NA \\
\hline A_24_P941759 & G2E3 & -0.2988 & 0.000386 & 0.019195 & -5.6742 & Down & G2/M-phase specific E3 ubiquitin protein ligase \\
\hline A_33_P3237364 & RPL36P20 & -0.39151 & 0.000388 & 0.019229 & -5.67001 & Down & ribosomal protein L36 pseudogene 20 \\
\hline A_33_P3374030 & LOC100132197 & -0.31421 & 0.000393 & 0.019342 & -5.65903 & Down & uncharacterized LOC100132197 \\
\hline $\begin{array}{l}\text { A_19_P00807621 } \\
\text { ERCC- }\end{array}$ & LINC01355 & -0.3223 & 0.000403 & 0.019654 & -5.63861 & Down & long intergenic non-protein coding RNA 1355 \\
\hline 00085_231 & \#N/A & -0.8721 & 0.000403 & 0.019654 & -5.63845 & Down & NA \\
\hline A_33_P3346643 & \#N/A & -0.65369 & 0.000403 & 0.019654 & -5.63821 & Down & NA \\
\hline A_33_P3342469 & OR6T1 & -0.39762 & 0.000405 & 0.019687 & -5.63402 & Down & olfactory receptor family 6 subfamily T member 1 \\
\hline A_23_P218827 & POLQ & -0.61577 & 0.000406 & 0.019694 & -5.63275 & Down & DNA polymerase theta \\
\hline A_33_P3268734 & COL5A1-AS1 & -0.30006 & 0.000411 & 0.019894 & -5.62161 & Down & $\begin{array}{l}\text { COL5A1 antisense RNA } 1 \\
\text { glutamate ionotropic receptor kainate type subunit }\end{array}$ \\
\hline A_23_P250136 & GRIK2 & -0.50891 & 0.000412 & 0.019894 & -5.61973 & Down & 2 \\
\hline A_33_P3398411 & RASSF3 & -0.78014 & 0.000419 & 0.020052 & -5.60582 & Down & Ras association domain family member 3 \\
\hline A_19_P00324378 & \#N/A & -1.09872 & 0.000425 & 0.020173 & -5.59533 & Down & NA \\
\hline A_19_P00326161 & \#N/A & -0.31777 & 0.000432 & 0.020315 & -5.58176 & Down & NA \\
\hline A_32_P62997 & PBK & -1.44872 & 0.000433 & 0.020315 & -5.58052 & Down & PDZ binding kinase \\
\hline A_33_P3304452 & SLC12A5-AS1 & -0.71833 & 0.000433 & 0.020315 & -5.57988 & Down & SLC12A5 and MMP9 antisense RNA 1 \\
\hline A_24_P413884 & CENPA & -1.04472 & 0.000435 & 0.020351 & -5.57587 & Down & centromere protein $\mathrm{A}$ \\
\hline A_33_P3335576 & \#N/A & -0.30201 & 0.000436 & 0.020351 & -5.57466 & Down & NA \\
\hline A_32_P470728 & GSX1 & -0.37056 & 0.000443 & 0.020438 & -5.56016 & Down & GS homeobox 1 \\
\hline A_19_P00804762 & \#N/A & -0.48298 & 0.000449 & 0.02053 & -5.55088 & Down & NA \\
\hline A_32_P233950 & DPPA5 & -0.82268 & 0.000449 & 0.02053 & -5.55041 & Down & developmental pluripotency associated 5 \\
\hline A_33_P3418185 & LYNX1 & -0.5229 & 0.000457 & 0.02064 & -5.53525 & Down & Ly6/neurotoxin 1 \\
\hline A_33_P3360271 & $\mathrm{EVX} 2$ & -0.52194 & 0.000458 & 0.02064 & -5.53386 & Down & even-skipped homeobox 2 \\
\hline A_24_P690811 & ASPDH & -0.41954 & 0.000463 & 0.020678 & -5.52511 & Down & aspartate dehydrogenase domain containing \\
\hline A_33_P3228709 & KRTAP5-7 & -0.35642 & 0.000464 & 0.020678 & -5.52349 & Down & keratin associated protein 5-7 \\
\hline A_19_P00331839 & \#N/A & -0.29212 & 0.00047 & 0.020745 & -5.51346 & Down & NA \\
\hline A_19_P00331107 & \#N/A & -0.80169 & 0.000474 & 0.020853 & -5.50635 & Down & NA \\
\hline A_23_P88331 & DLGAP5 & -0.90972 & 0.000475 & 0.020853 & -5.50474 & Down & DLG associated protein 5 \\
\hline A_33_P3258392 & EDN1 & -0.3889 & 0.000475 & 0.020853 & -5.50367 & Down & endothelin 1 \\
\hline A_19_P00804522 & \#N/A & -0.57 & 0.000476 & 0.020853 & -5.50304 & Down & NA \\
\hline A_19_P00317565 & \#N/A & -0.46065 & 0.000476 & 0.020853 & -5.50268 & Down & NA \\
\hline A_19_P00802508 & \#N/A & -0.59414 & 0.000477 & 0.020888 & -5.49991 & Down & NA \\
\hline A_19_P00803716 & \#N/A & -0.79278 & 0.000484 & 0.021054 & -5.4892 & Down & NA \\
\hline A_19_P00320023 & \#N/A & -0.53921 & 0.000495 & 0.021302 & -5.47115 & Down & NA \\
\hline A_19_P00320253 & \#N/A & -0.4143 & 0.000504 & 0.021479 & -5.45555 & Down & NA \\
\hline A_19_P00315793 & AL138781.1 & -0.49995 & 0.000507 & 0.021538 & -5.45163 & Down & NA \\
\hline A_19_P00320113 & \#N/A & -1.04798 & 0.000508 & 0.021586 & -5.44901 & Down & NA \\
\hline A_19_P00322033 & \#N/A & -0.67146 & 0.000518 & 0.021761 & -5.43368 & Down & NA \\
\hline
\end{tabular}




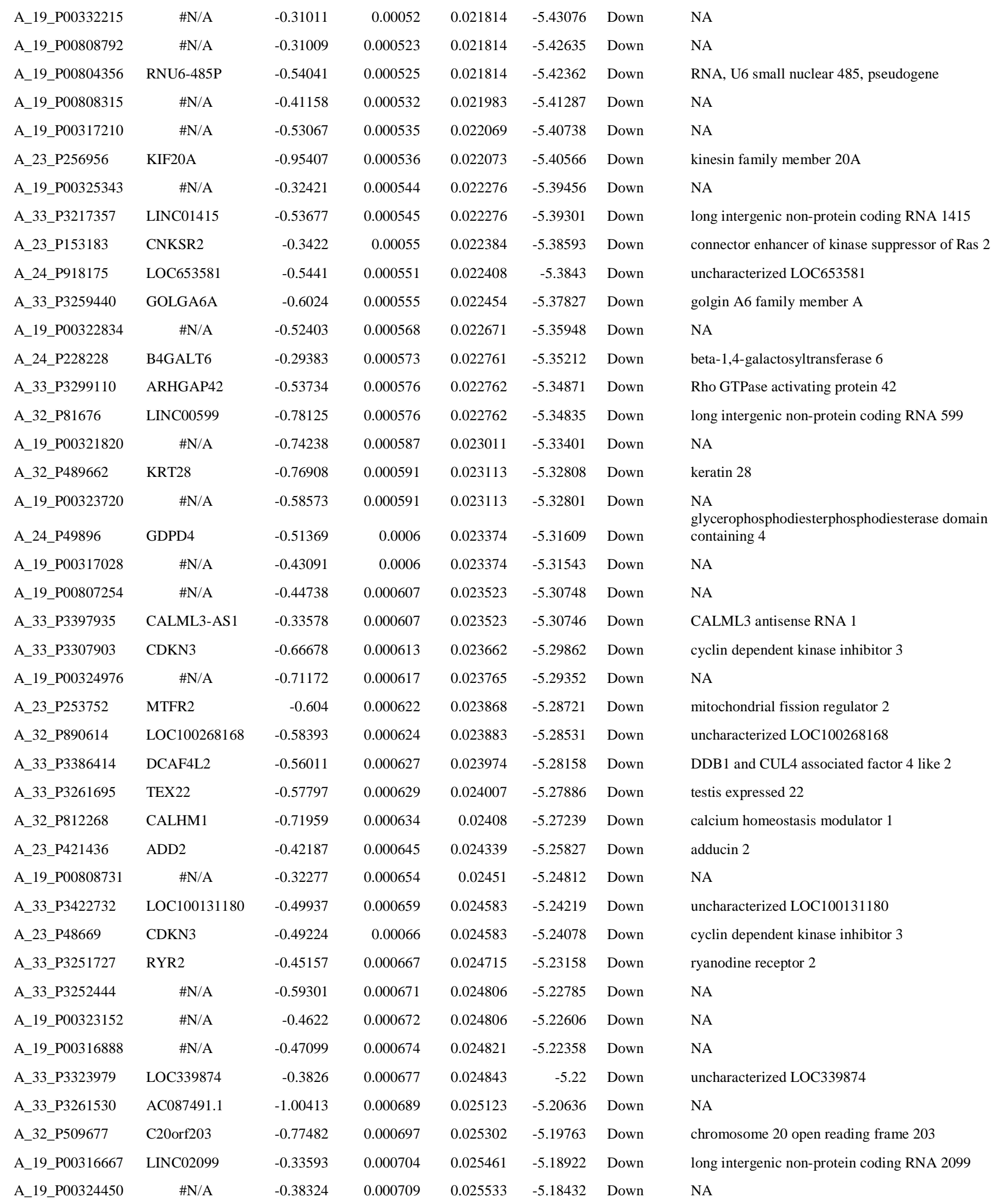




\begin{tabular}{|c|c|c|c|c|c|c|c|}
\hline A_33_P3407103 & YTHDF1 & -0.33159 & 0.000724 & 0.025806 & -5.1674 & Down & $\begin{array}{l}\text { YTH N6-methyladenosine RNA binding protein } 1 \\
\text { RNA binding motif protein, Y-linked, family } 1 \text {, } \\
\text { member B }\end{array}$ \\
\hline A_33_P3348328 & \#N/A & -0.45257 & 0.000727 & 0.02587 & -5.16416 & Down & NA \\
\hline A_23_P151150 & FOXM1 & -0.63036 & 0.000731 & 0.025919 & -5.16005 & Down & forkhead box M1 \\
\hline A_19_P00811940 & \#N/A & -0.41876 & 0.000733 & 0.025954 & -5.15836 & Down & NA \\
\hline A_19_P00800868 & \#N/A & -0.32832 & 0.000751 & 0.026166 & -5.13882 & Down & NA \\
\hline A_23_P1083 & GJA4 & -0.54207 & 0.000752 & 0.026166 & -5.1378 & Down & gap junction protein alpha 4 \\
\hline A_33_P3217619 & AC078923.1 & -0.55897 & 0.000782 & 0.026859 & -5.10793 & Down & NA \\
\hline A_33_P3298173 & DEFA7P & -0.44812 & 0.000783 & 0.026893 & -5.10632 & Down & defensin alpha 7 , pseudogene \\
\hline A_24_P79403 & PF4 & -0.6126 & 0.000785 & 0.026893 & -5.10463 & Down & platelet factor 4 \\
\hline A_33_P3300142 & ANKFY1 & -0.30215 & 0.000785 & 0.026893 & -5.10422 & Down & ankyrin repeat and FYVE domain containing 1 \\
\hline A_23_P14035 & AKAP3 & -0.92292 & 0.000792 & 0.026979 & -5.09727 & Down & A-kinase anchoring protein 3 \\
\hline A_32_P168349 & \#N/A & -0.4856 & 0.000793 & 0.026979 & -5.09635 & Down & NA \\
\hline A_33_P3309999 & DCAF12L2 & -0.32282 & 0.000797 & 0.027003 & -5.09317 & Down & DDB1 and CUL4 associated factor 12 like 2 \\
\hline A_32_P7316 & BDNF & -1.4327 & 0.000798 & 0.02703 & -5.09156 & Down & brain derived neurotrophic factor \\
\hline A_19_P00323487 & \#N/A & -0.33195 & 0.000802 & 0.027092 & -5.08752 & Down & NA \\
\hline A_23_P118815 & BIRC5 & -0.45791 & 0.000808 & 0.027224 & -5.08189 & Down & baculoviral IAP repeat containing 5 \\
\hline A_19_P00318567 & \#N/A & -0.43335 & 0.000816 & 0.027426 & -5.07411 & Down & NA \\
\hline A_33_P3385006 & SLC39A5 & -0.44216 & 0.000817 & 0.027426 & -5.0737 & Down & solute carrier family 39 member 5 \\
\hline A_19_P00809507 & \#N/A & -0.98202 & 0.00082 & 0.027463 & -5.0702 & Down & NA \\
\hline A_19_P00321075 & PVT1 & -1.26911 & 0.000829 & 0.02766 & -5.06179 & Down & Pvt1 oncogene (non-protein coding) \\
\hline A_33_P3349265 & GLYATL3 & -0.50093 & 0.000838 & 0.027779 & -5.05349 & Down & glycine- $\mathrm{N}$-acyltransferase like 3 \\
\hline A_33_P3374443 & L1CAM & -0.56749 & 0.000842 & 0.027859 & -5.05006 & Down & L1 cell adhesion molecule \\
\hline A_33_P3314301 & SV2C & -0.47211 & 0.000843 & 0.027861 & -5.04938 & Down & synaptic vesicle glycoprotein $2 \mathrm{C}$ \\
\hline A_23_P80902 & KIF15 & -0.4448 & 0.000853 & 0.028079 & -5.04014 & Down & kinesin family member 15 \\
\hline A_23_P143994 & FANCD2 & -0.60919 & 0.000858 & 0.02818 & -5.03582 & Down & Fanconianemia complementation group D2 \\
\hline A_19_P00319532 & LINC00963 & -0.30593 & 0.00086 & 0.028195 & -5.03397 & Down & long intergenic non-protein coding RNA 963 \\
\hline A_32_P186474 & RACGAP1 & -0.42374 & 0.000861 & 0.028195 & -5.03302 & Down & $\begin{array}{l}\text { RacGTPase activating protein } 1 \\
\text { FAM20C, golgi associated secretory pathway }\end{array}$ \\
\hline A_33_P3346635 & FAM20C & -0.51837 & 0.000867 & 0.028288 & -5.02725 & Down & kinase \\
\hline A_23_P74349 & NUF2 & -0.61002 & 0.000873 & 0.028388 & -5.0222 & Down & NUF2, NDC80 kinetochore complex component \\
\hline A_19_P00321690 & AL390755.1 & -0.8147 & 0.000878 & 0.02848 & -5.01818 & Down & NA \\
\hline A_19_P00324561 & \#N/A & -0.30458 & 0.000889 & 0.028637 & -5.00807 & Down & NA \\
\hline A_33_P3354783 & \#N/A & -0.43482 & 0.000901 & 0.028915 & -4.99774 & Down & NA \\
\hline A_33_P3220475 & QSOX1 & -0.34597 & 0.00091 & 0.0291 & -4.99053 & Down & quiescin sulfhydryl oxidase 1 \\
\hline A_19_P00316565 & TSIX & -0.70329 & 0.000918 & 0.029195 & -4.9834 & Down & TSIX transcript, XIST antisense RNA \\
\hline A_33_P3230189 & SLITRK6 & -0.43506 & 0.000921 & 0.029279 & -4.98064 & Down & SLIT and NTRK like family member 6 \\
\hline A_19_P00323540 & \#N/A & -0.29165 & 0.000924 & 0.029301 & -4.97835 & Down & NA \\
\hline A_33_P3591761 & FAM91A1 & -0.29173 & 0.000929 & 0.029399 & -4.97455 & Down & family with sequence similarity 91 member A1 \\
\hline A_19_P00324420 & H19_1 & -0.3534 & 0.000939 & 0.029592 & -4.9662 & Down & H19 conserved region 1 \\
\hline A_33_P3269854 & AL662860.1 & -0.35472 & 0.00094 & 0.029592 & -4.96563 & Down & NA \\
\hline A_19_P00315768 & \#N/A & -0.36314 & 0.000942 & 0.02963 & -4.96409 & Down & NA \\
\hline A_23_P103532 & GPR161 & -0.44277 & 0.000943 & 0.029642 & -4.96303 & Down & G protein-coupled receptor 161 \\
\hline
\end{tabular}




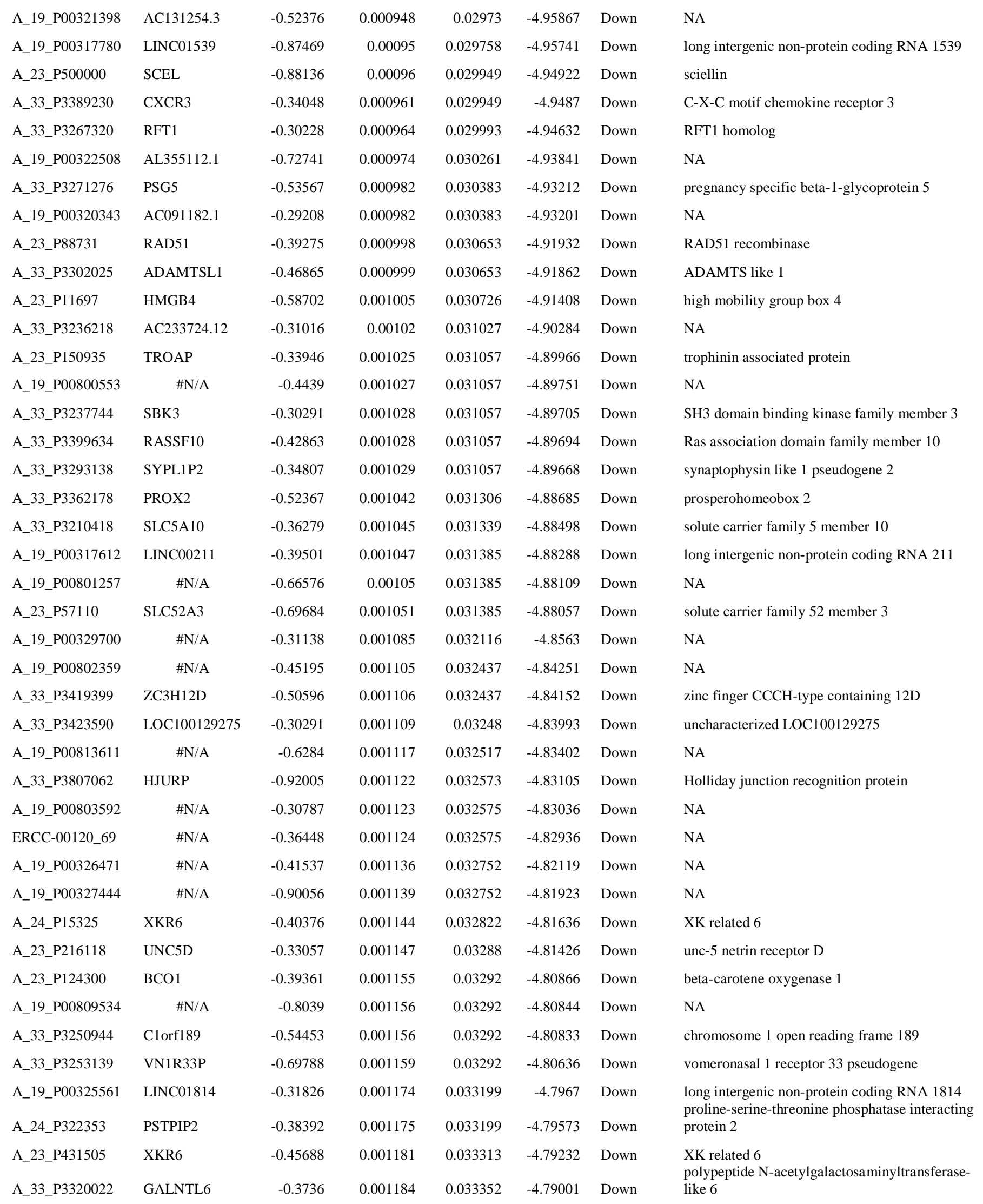




\begin{tabular}{|c|c|c|c|c|c|c|c|}
\hline A_19_P00810308 & \#N/A & -0.52859 & 0.001185 & 0.033352 & -4.78995 & Down & NA \\
\hline A_23_P376036 & EXD1 & -0.69087 & 0.001197 & 0.033513 & -4.7819 & Down & $\begin{array}{l}\text { exonuclease } 3 \text { '-5' domain containing } 1 \\
\text { spermatogenesis associated } 42 \text { (non-protein }\end{array}$ \\
\hline A_32_P70056 & SPATA42 & -1.07945 & 0.001203 & 0.033572 & -4.77858 & Down & coding) \\
\hline A_24_P924816 & SLC2A13 & -0.83023 & 0.001211 & 0.033718 & -4.77338 & Down & solute carrier family 2 member 13 \\
\hline A_19_P00326183 & \#N/A & -0.50802 & 0.001214 & 0.033752 & -4.77163 & Down & NA \\
\hline A_23_P126888 & KIF21B & -0.35514 & 0.001222 & 0.033852 & -4.76647 & Down & kinesin family member $21 \mathrm{~B}$ \\
\hline A_33_P3252083 & GRIP1 & -0.30525 & 0.001235 & 0.03403 & -4.75838 & Down & glutamate receptor interacting protein 1 \\
\hline A_33_P3231908 & POU3F3 & -1.22314 & 0.001245 & 0.034205 & -4.75272 & Down & POU class 3 homeobox 3 \\
\hline A_23_P305245 & C3orf80 & -0.92969 & 0.001246 & 0.034205 & -4.75191 & Down & chromosome 3 open reading frame 80 \\
\hline A_23_P98147 & CPN1 & -0.39421 & 0.00125 & 0.034238 & -4.74955 & Down & carboxypeptidase $\mathrm{N}$ subunit 1 \\
\hline A_23_P51085 & SPC25 & -0.89359 & 0.001265 & 0.0345 & -4.74067 & Down & SPC25, NDC80 kinetochore complex component \\
\hline A_33_P3294911 & C12ORF66 & -0.75124 & 0.001272 & 0.03457 & -4.73677 & Down & chromosome 12 open reading frame 66 \\
\hline A_23_P94422 & MELK & -1.00289 & 0.001289 & 0.034824 & -4.72646 & Down & maternal embryonic leucine zipper kinase \\
\hline A_23_P94494 & TLE4 & -0.40005 & 0.00129 & 0.034824 & -4.7262 & Down & transducin like enhancer of split 4 \\
\hline A_23_P133123 & MND1 & -0.49815 & 0.00129 & 0.034824 & -4.72607 & Down & meiotic nuclear divisions 1 \\
\hline A_23_P113283 & ZMAT3 & -0.30962 & 0.001308 & 0.035031 & -4.71552 & Down & zinc finger matrin-type 3 \\
\hline A_19_P00321002 & \#N/A & -0.66499 & 0.001315 & 0.035072 & -4.71182 & Down & $\begin{array}{l}\text { NA } \\
\text { ATPase sarcoplasmic/endoplasmic reticulum }\end{array}$ \\
\hline A_24_P202319 & ATP2A3 & -0.53341 & 0.001317 & 0.03508 & -4.71075 & Down & $\mathrm{Ca} 2+$ transporting 3 \\
\hline A_19_P00808287 & \#N/A & -0.58374 & 0.001319 & 0.035104 & -4.70957 & Down & NA \\
\hline A_19_P00322255 & $\mathrm{AC} 027031.2$ & -0.30074 & 0.001324 & 0.035191 & -4.70652 & Down & NA \\
\hline A_23_P113382 & GRIP1 & -0.48946 & 0.00133 & 0.035335 & -4.70303 & Down & glutamate receptor interacting protein 1 \\
\hline A_33_P3387831 & CENPM & -0.34165 & 0.001335 & 0.03541 & -4.70031 & Down & centromere protein $\mathrm{M}$ \\
\hline A_19_P00812133 & \#N/A & -0.46632 & 0.001337 & 0.035432 & -4.69913 & Down & $\begin{array}{l}\text { NA } \\
\text { glutamate ionotropic receptor kainate type subunit }\end{array}$ \\
\hline A_33_P3307133 & GRIK2 & -0.39228 & 0.00134 & 0.035451 & -4.6979 & Down & 2 \\
\hline A_33_P3265872 & AC002454.1 & -0.41286 & 0.001341 & 0.035467 & -4.697 & Down & NA \\
\hline A_33_P3233085 & AL049775.3 & -0.76663 & 0.001343 & 0.035471 & -4.69629 & Down & NA \\
\hline A_33_P3229276 & ZSCAN2 & -0.34477 & 0.001347 & 0.035471 & -4.69368 & Down & zinc finger and SCAN domain containing 2 \\
\hline A_23_P305581 & GPR26 & -0.92643 & 0.001354 & 0.035473 & -4.68974 & Down & G protein-coupled receptor 26 \\
\hline A_33_P3363245 & NXPH4 & -0.59888 & 0.001356 & 0.035473 & -4.68905 & Down & neurexophilin 4 \\
\hline A_33_P3378972 & UNC5D & -0.48731 & 0.001364 & 0.035577 & -4.68463 & Down & unc-5 netrin receptor $\mathrm{D}$ \\
\hline A_19_P00315659 & \#N/A & -0.50683 & 0.001364 & 0.035577 & -4.68448 & Down & NA \\
\hline A_33_P3301291 & FOXB2 & -0.65239 & 0.001367 & 0.035577 & -4.68318 & Down & forkhead box B2 \\
\hline A_33_P3273732 & BX649632.1 & -0.29717 & 0.001374 & 0.035707 & -4.67918 & Down & NA \\
\hline A_33_P3354291 & RNF215 & -0.40503 & 0.001375 & 0.035711 & -4.67845 & Down & ring finger protein 215 \\
\hline A_19_P00320972 & STMN1 & -0.29389 & 0.001376 & 0.035711 & -4.67818 & Down & stathmin 1 \\
\hline A_23_P202894 & ACRV1 & -0.7613 & 0.00138 & 0.035747 & -4.67571 & Down & acrosomal vesicle protein 1 \\
\hline A_33_P3212316 & \#N/A & -0.3866 & 0.00138 & 0.035747 & -4.67563 & Down & NA \\
\hline A_19_P00317630 & AC007221.1 & -0.88118 & 0.001395 & 0.036006 & -4.668 & Down & NA \\
\hline A_19_P00328506 & \#N/A & -0.31695 & 0.001419 & 0.036362 & -4.65535 & Down & NA \\
\hline A_23_P422933 & ARHGAP20 & -0.36989 & 0.001429 & 0.036533 & -4.64983 & Down & Rho GTPase activating protein 20 \\
\hline A_32_P151800 & FAM72D & -0.61437 & 0.00144 & 0.036674 & -4.64458 & Down & family with sequence similarity 72 member D \\
\hline A_19_P00810205 & $\mathrm{CCNB} 2 \mathrm{P} 1$ & -0.39557 & 0.001449 & 0.036853 & -4.63977 & Down & cyclin B2 pseudogene 1 \\
\hline
\end{tabular}




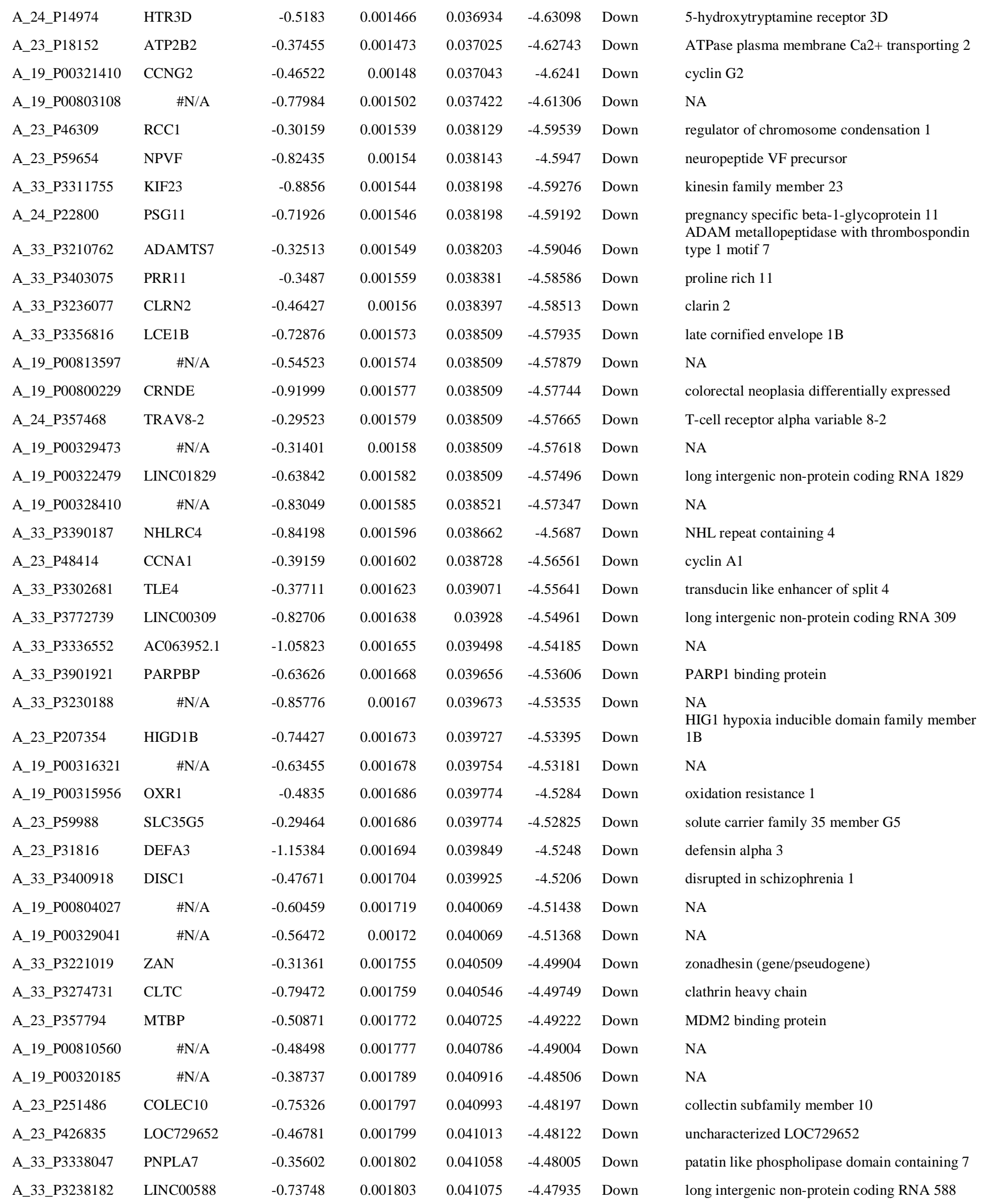




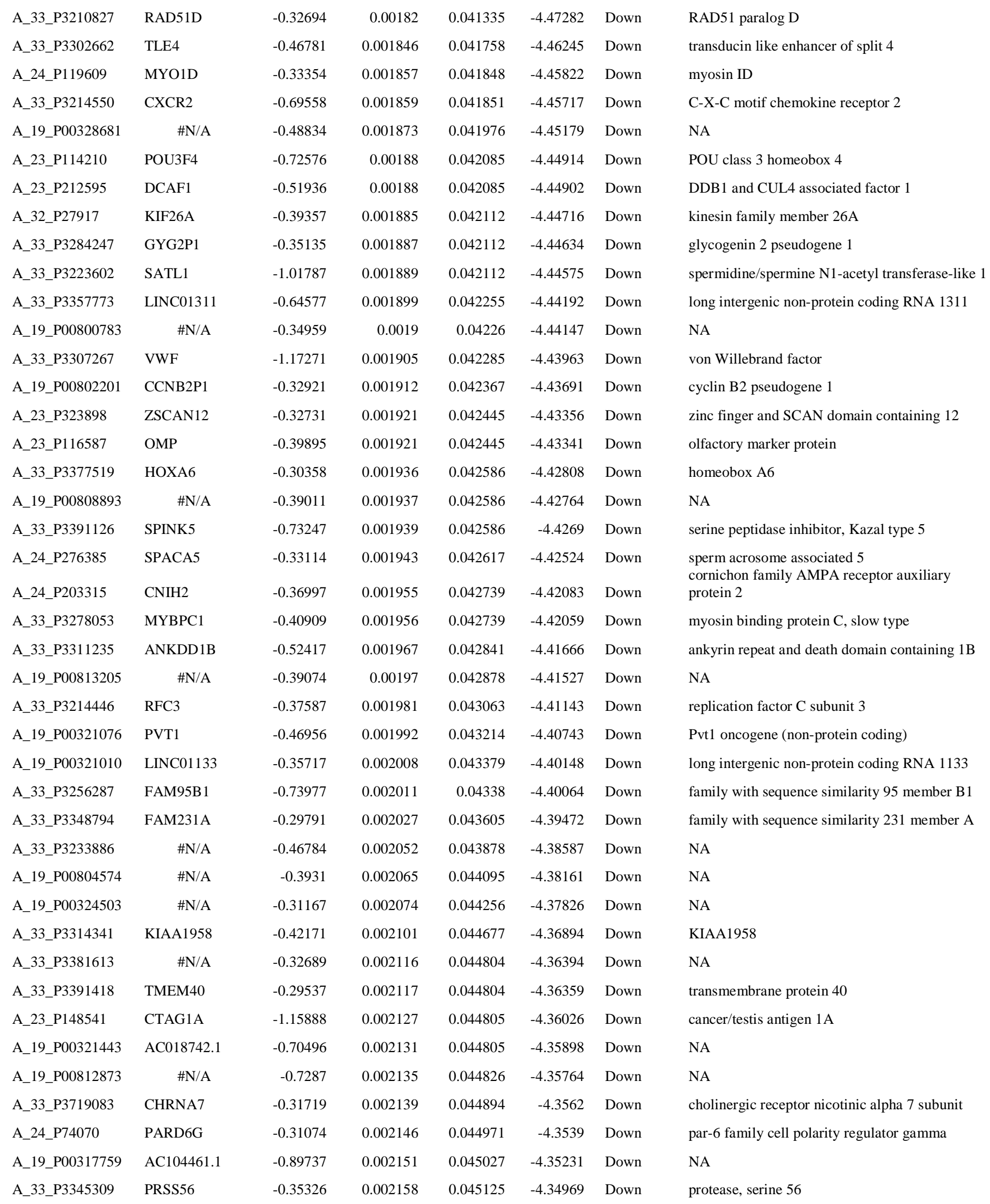




\begin{tabular}{|c|c|c|c|c|c|c|c|}
\hline A_19_P00804601 & TSSC2 & -0.58666 & 0.002163 & 0.045173 & -4.34823 & Down & $\begin{array}{l}\text { tumor suppressing subtransferable candidate } 2 \\
\text { pseudogene }\end{array}$ \\
\hline A_19_P00329437 & \#N/A & -0.59541 & 0.002165 & 0.045202 & -4.34741 & Down & NA \\
\hline A_23_P429491 & DDIAS & -0.4245 & 0.002191 & 0.045511 & -4.33903 & Down & DNA damage induced apoptosis suppressor \\
\hline A_32_P196115 & ZSCAN25 & -0.29268 & 0.002196 & 0.045572 & -4.33738 & Down & zinc finger and SCAN domain containing 25 \\
\hline A_23_P208240 & ZNF160 & -0.37222 & 0.002215 & 0.045759 & -4.33107 & Down & zinc finger protein 160 \\
\hline A_19_P00326743 & AL121655.1 & -0.3691 & 0.002223 & 0.045831 & -4.32845 & Down & NA \\
\hline A_33_P3407631 & AC133565.1 & -0.65338 & 0.002226 & 0.045834 & -4.32755 & Down & NA \\
\hline A_33_P3296181 & CCL3L3 & -0.572 & 0.002227 & 0.045834 & -4.32736 & Down & C-C motif chemokine ligand 3 like 3 \\
\hline A_19_P00802881 & \#N/A & -0.41452 & 0.002228 & 0.045845 & -4.32685 & Down & NA \\
\hline A_19_P00324996 & \#N/A & -0.29106 & 0.002233 & 0.045897 & -4.32536 & Down & NA \\
\hline A_32_P62090 & $\mathrm{CCDC} 182$ & -0.31268 & 0.00225 & 0.046061 & -4.32007 & Down & $\begin{array}{l}\text { coiled-coil domain containing } 182 \\
\text { transcription elongation factor A N-terminal and }\end{array}$ \\
\hline A_33_P3267270 & TCEANC2 & -0.33479 & 0.002255 & 0.04608 & -4.31822 & Down & central domain containing 2 \\
\hline A_23_P71727 & CKS2 & -0.30604 & 0.002256 & 0.04608 & -4.31802 & Down & CDC28 protein kinase regulatory subunit 2 \\
\hline A_32_P120791 & LOC727916 & -0.38371 & 0.002261 & 0.04611 & -4.31653 & Down & uncharacterized LOC727916 \\
\hline A_23_P51580 & HSD3B2 & -0.56617 & 0.002263 & 0.046111 & -4.31586 & Down & and steroid delta-isomerase 2 \\
\hline A_33_P3415156 & \#N/A & -0.44703 & 0.002272 & 0.046219 & -4.31314 & Down & NA \\
\hline A_33_P3419567 & RAD21L1 & -0.49999 & 0.00228 & 0.046295 & -4.31051 & Down & RAD21 cohesin complex component like 1 \\
\hline A_23_P118158 & HS3ST2 & -0.78188 & 0.002283 & 0.046327 & -4.30943 & Down & heparansulfate-glucosamine 3-sulfotransferase 2 \\
\hline A_24_P83899 & GRM5 & -0.6979 & 0.002291 & 0.046451 & -4.30718 & Down & $\begin{array}{l}\text { glutamate metabotropic receptor } 5 \\
\text { olfactory receptor family } 5 \text { subfamily } \mathrm{M} \text { member }\end{array}$ \\
\hline A_33_P3343496 & OR5M1 & -1.13651 & 0.002299 & 0.046535 & -4.30454 & Down & 1 \\
\hline A_23_P252388 & SPEF2 & -0.34362 & 0.002329 & 0.046876 & -4.29526 & Down & sperm flagellar 2 \\
\hline A_33_P3254579 & KRTAP1-5 & -0.53907 & 0.002339 & 0.04696 & -4.29225 & Down & $\begin{array}{l}\text { keratin associated protein } 1-5 \\
\text { killer cell immunoglobulin like receptor, three Ig }\end{array}$ \\
\hline A_33_P3340926 & KIR3DX1 & -0.35799 & 0.002354 & 0.047043 & -4.28758 & Down & domains X1 \\
\hline A_33_P3305456 & SSU72P2 & -0.8223 & 0.002355 & 0.047043 & -4.28742 & Down & SSU72 pseudogene 2 \\
\hline A_33_P3281041 & \#N/A & -0.30238 & 0.002357 & 0.047054 & -4.28686 & Down & NA \\
\hline A_24_P944973 & EYS & -0.99854 & 0.002366 & 0.047061 & -4.28409 & Down & eyes shut homolog (Drosophila) \\
\hline A_33_P3278896 & \#N/A & -0.3572 & 0.002381 & 0.047187 & -4.27953 & Down & NA \\
\hline A_33_P3315979 & OR4Q3 & -0.71413 & 0.002409 & 0.047533 & -4.27135 & Down & olfactory receptor family 4 subfamily Q member 3 \\
\hline A_19_P00320885 & AC091182.1 & -0.34391 & 0.002412 & 0.04755 & -4.27044 & Down & NA \\
\hline A_33_P3311676 & LINC00703 & -0.70239 & 0.002438 & 0.047883 & -4.26282 & Down & long intergenic non-protein coding RNA 703 \\
\hline A_33_P3363690 & LINC01182 & -0.7252 & 0.002439 & 0.047883 & -4.26253 & Down & $\begin{array}{l}\text { long intergenic non-protein coding RNA } 1182 \\
\text { olfactory receptor family } 13 \text { subfamily J member }\end{array}$ \\
\hline A_33_P3213982 & OR13J1 & -0.85672 & 0.002442 & 0.0479 & -4.26162 & Down & 1 \\
\hline A_23_P401024 & LINC00304 & -0.35474 & 0.002447 & 0.047944 & -4.26028 & Down & $\begin{array}{l}\text { long intergenic non-protein coding RNA } 304 \\
\text { MEF2 activating motif and SAP domain }\end{array}$ \\
\hline A_24_P297537 & MAMSTR & -0.40407 & 0.002451 & 0.047944 & -4.2591 & Down & containing transcriptional regulator \\
\hline A_23_P110802 & CENPH & -0.52312 & 0.002457 & 0.047944 & -4.25737 & Down & centromere protein $\mathrm{H}$ \\
\hline A_23_P58009 & C3orf52 & -0.46294 & 0.002461 & 0.047975 & -4.25616 & Down & chromosome 3 open reading frame 52 \\
\hline A_24_P472007 & UNC13A & -0.32027 & 0.002474 & 0.048119 & -4.25251 & Down & unc-13 homolog A \\
\hline A_24_P221485 & PSAT1P3 & -0.97228 & 0.002488 & 0.04829 & -4.2483 & Down & phosphoserine aminotransferase 1 pseudogene \\
\hline A_33_P3298267 & RUFY4 & -0.33366 & 0.002489 & 0.04829 & -4.24805 & Down & RUN and FYVE domain containing 4 \\
\hline A_33_P3367606 & FLJ13224 & -0.47084 & 0.002493 & 0.048337 & -4.24705 & Down & uncharacterized LOC79857 \\
\hline A_24_P361427 & TBC1D29 & -0.37791 & 0.002515 & 0.048568 & -4.24082 & Down & TBC1 domain family member 29 \\
\hline
\end{tabular}




\begin{tabular}{|c|c|c|c|c|c|c|c|}
\hline A_33_P3256317 & MYT1L-AS1 & -0.71248 & 0.002517 & 0.048568 & -4.24024 & Down & MYT1L antisense RNA 1 \\
\hline A_19_P00317146 & AC006116.8 & -0.29745 & 0.00252 & 0.048568 & -4.23944 & Down & NA \\
\hline A_33_P3236846 & GPR17 & -0.61794 & 0.002521 & 0.048568 & -4.23892 & Down & G protein-coupled receptor 17 \\
\hline A_33_P3372376 & \#N/A & -0.48123 & 0.002522 & 0.048568 & -4.2387 & Down & NA \\
\hline A_33_P3328921 & LINC00596 & -0.29513 & 0.002524 & 0.048568 & -4.23823 & Down & long intergenic non-protein coding RNA 596 \\
\hline A_19_P00315998 & \#N/A & -1.11027 & 0.002554 & 0.048927 & -4.22988 & Down & NA \\
\hline A_33_P3417474 & CR381653.2 & -1.16255 & 0.002554 & 0.048927 & -4.22977 & Down & NA \\
\hline A_33_P3419577 & RAD21L1 & -0.45966 & 0.002558 & 0.04897 & -4.22884 & Down & RAD21 cohesin complex component like 1 \\
\hline A_19_P00801549 & \#N/A & -0.40986 & 0.00258 & 0.049254 & -4.22271 & Down & NA \\
\hline A_33_P3325330 & \#N/A & -0.6559 & 0.002582 & 0.049254 & -4.2222 & Down & $\begin{array}{l}\text { NA } \\
\text { olfactory receptor family } 11 \text { subfamily } \mathrm{H} \text { member }\end{array}$ \\
\hline A_33_P3292565 & OR11H7 & -0.35919 & 0.002597 & 0.049431 & -4.21804 & Down & $\begin{array}{l}7 \text { (gene/pseudogene) } \\
\text { calcium/calmodulin dependent serine protein }\end{array}$ \\
\hline A_33_P3244096 & CASK & -0.39817 & 0.002618 & 0.049598 & -4.2122 & Down & kinase \\
\hline A_33_P3414107 & $\mathrm{AC} 123595.1$ & -0.86069 & 0.002619 & 0.049598 & -4.21197 & Down & NA \\
\hline A_19_P00322418 & OXR1 & -0.41218 & 0.002621 & 0.049598 & -4.21162 & Down & oxidation resistance 1 \\
\hline A_19_P00802684 & AC009413.1 & -0.64286 & 0.002634 & 0.049766 & -4.20802 & Down & NA \\
\hline A_33_P3401397 & C7orf65 & -0.62876 & 0.002646 & 0.049904 & -4.20474 & Down & chromosome 7 open reading frame 65 \\
\hline
\end{tabular}

NA- not annotated

Table 2 The enriched pathway terms of the up-regulated differentially expressed genes BIOCYC

\begin{tabular}{|c|c|c|c|c|c|c|c|}
\hline $\begin{array}{l}\text { Pathw } \\
\text { ay ID }\end{array}$ & Pathway Name & P-value & $\begin{array}{l}\text { FDR } \\
\text { B\&H }\end{array}$ & $\begin{array}{l}\text { FDR } \\
\text { B\&Y }\end{array}$ & Bonferroni & $\begin{array}{l}\text { Gene } \\
\text { Count }\end{array}$ & Gene \\
\hline 545296 & retinoate biosynthesis II & $1.62 \mathrm{E}-03$ & $4.52 \mathrm{E}-02$ & $1.78 \mathrm{E}-01$ & $4.52 \mathrm{E}-02$ & 02 & XDH,RBP4 \\
\hline 545295 & retinoate biosynthesis I & $7.21 \mathrm{E}-03$ & $1.01 \mathrm{E}-01$ & $3.97 \mathrm{E}-01$ & 2.02E-01 & 02 & ALDH1A3,RBP4 \\
\hline 142437 & nicotine degradation IV & $1.13 \mathrm{E}-02$ & $1.06 \mathrm{E}-01$ & 4.16E-01 & $3.18 \mathrm{E}-01$ & 02 & FMO5,AOX1 \\
\hline 545294 & $\begin{array}{l}\text { the visual cycle I } \\
\text { (vertebrates) }\end{array}$ & $2.51 \mathrm{E}-02$ & $1.54 \mathrm{E}-01$ & $6.05 \mathrm{E}-01$ & 7.02E-01 & 02 & LRAT,RBP4 \\
\hline 545293 & retinol biosynthesis & $3.18 \mathrm{E}-02$ & $1.54 \mathrm{E}-01$ & $6.05 \mathrm{E}-01$ & 8.89E-01 & 02 & LRAT,RBP4 \\
\hline 142238 & catecholamine biosynthesis & $3.30 \mathrm{E}-02$ & $1.54 \mathrm{E}-01$ & $6.05 \mathrm{E}-01$ & $9.24 \mathrm{E}-01$ & 01 & PNMT \\
\hline 545318 & L-carnitine biosynthesis & 4.91E-02 & $1.72 \mathrm{E}-01$ & $6.74 \mathrm{E}-01$ & $1.00 \mathrm{E}+00$ & 01 & BBOX1 \\
\hline 545363 & $\begin{array}{l}\text { guanosine nucleotides } \\
\text { degradation }\end{array}$ & 4.91E-02 & $1.72 \mathrm{E}-01$ & $6.74 \mathrm{E}-01$ & $1.00 \mathrm{E}+00$ & 01 & $\mathrm{XDH}$ \\
\hline
\end{tabular}




\section{KEGG}

\begin{tabular}{|c|c|c|c|c|c|c|c|}
\hline 1026136 & $\begin{array}{l}\text { Signaling pathways } \\
\text { regulating pluripotency of } \\
\text { stem cells }\end{array}$ & $5.18 \mathrm{E}-04$ & $5.12 \mathrm{E}-02$ & $2.94 \mathrm{E}-01$ & 8.97E-02 & 09 & $\begin{array}{l}\text { FGFR3,FGFR2,WNT5A,ID } \\
\text { 4,SOX2,INHBA,LEFTY2,P } \\
\text { AX6,SMAD9 }\end{array}$ \\
\hline 83053 & $\begin{array}{l}\text { Neuroactive ligand- } \\
\text { receptor interaction }\end{array}$ & $7.68 \mathrm{E}-04$ & $5.12 \mathrm{E}-02$ & 2.94E-01 & $1.33 \mathrm{E}-01$ & 13 & $\begin{array}{l}\text { TSHB,CSH1,CSH2,FSHB,G } \\
\text { ALR1,SSTR2,GH1,GH2,G } \\
\text { HRHR,KISS1R,GNRHR,CC } \\
\text { KBR,P2RY2 }\end{array}$ \\
\hline 83032 & $\begin{array}{l}\text { Drug metabolism - } \\
\text { cytochrome P450 }\end{array}$ & $1.06 \mathrm{E}-03$ & $5.12 \mathrm{E}-02$ & 2.94E-01 & $1.84 \mathrm{E}-01$ & 06 & $\begin{array}{l}\text { UGT2A2,MGST1,ALDH1A } \\
\text { 3,FMO5,AOX1,UGT2A1 }\end{array}$ \\
\hline 83077 & $\begin{array}{l}\text { Jak-STAT signaling } \\
\text { pathway }\end{array}$ & $1.18 \mathrm{E}-03$ & $5.12 \mathrm{E}-02$ & $2.94 \mathrm{E}-01$ & $2.05 \mathrm{E}-01$ & 09 & $\begin{array}{l}\text { AOX1,CSH1,CSH2,IFNE,I } \\
\text { L6R,IL20RA,IL22RA1,GH1 } \\
\text {,GH2 }\end{array}$ \\
\hline 83031 & $\begin{array}{l}\text { Metabolism of xenobiotics } \\
\text { by cytochrome P450 }\end{array}$ & $7.71 \mathrm{E}-03$ & $2.56 \mathrm{E}-01$ & $1.00 \mathrm{E}+00$ & $1.00 \mathrm{E}+00$ & 05 & $\begin{array}{l}\text { UGT2A2,MGST1,HSD11B } \\
\text { 1,ALDH1A3,UGT2A1 }\end{array}$ \\
\hline 83096 & $\begin{array}{l}\text { Maturity onset diabetes of } \\
\text { the young }\end{array}$ & $8.89 \mathrm{E}-03$ & $2.56 \mathrm{E}-01$ & $1.00 \mathrm{E}+00$ & $1.00 \mathrm{E}+00$ & 03 & NEUROD1,NKX2-2,PAX6 \\
\hline 673221 & Chemical carcinogenesis & $1.18 \mathrm{E}-02$ & $2.80 \mathrm{E}-01$ & $1.00 \mathrm{E}+00$ & $1.00 \mathrm{E}+00$ & 05 & $\begin{array}{l}\text { UGT2A2,MGST1,HSD11B } \\
\text { 1,ALDH1A3,UGT2A1 }\end{array}$ \\
\hline 83064 & $\begin{array}{l}\text { TGF-beta signaling } \\
\text { pathway }\end{array}$ & $1.29 \mathrm{E}-02$ & $2.80 \mathrm{E}-01$ & $1.00 \mathrm{E}+00$ & $1.00 \mathrm{E}+00$ & 05 & $\begin{array}{l}\text { ID4,INHBA,BMP7,LEFTY2 } \\
\text { „SMAD9 }\end{array}$ \\
\hline 82940 & $\begin{array}{l}\text { Steroid hormone } \\
\text { biosynthesis }\end{array}$ & $1.57 \mathrm{E}-02$ & $2.95 \mathrm{E}-01$ & $1.00 \mathrm{E}+00$ & $1.00 \mathrm{E}+00$ & 04 & $\begin{array}{l}\text { UGT2A2,HSD11B1,HSD17 } \\
\text { B2,UGT2A1 }\end{array}$ \\
\hline 172847 & $\begin{array}{l}\text { Protein digestion and } \\
\text { absorption }\end{array}$ & $1.70 \mathrm{E}-02$ & $2.95 \mathrm{E}-01$ & $1.00 \mathrm{E}+00$ & $1.00 \mathrm{E}+00$ & 05 & $\begin{array}{l}\text { COL4A6,COL13A1,SLC15 } \\
\text { A1,CTRB1,COL21A1 }\end{array}$ \\
\hline
\end{tabular}

Pathway Interaction Database

\begin{tabular}{llllllll}
\hline 137983 & ALK2 signaling events & $1.37 \mathrm{E}-02$ & $5.30 \mathrm{E}-01$ & $1.00 \mathrm{E}+00$ & $6.72 \mathrm{E}-01$ & 02 & BMP7,SMAD9 \\
\multirow{2}{*}{137948} & BMP receptor signaling & $2.16 \mathrm{E}-02$ & $5.30 \mathrm{E}-01$ & $1.00 \mathrm{E}+00$ & $1.00 \mathrm{E}+00$ & 03 & BMP7,CHRDL1,SMAD9
\end{tabular}

\section{REACTOME}

\begin{tabular}{|c|c|c|c|c|c|c|c|}
\hline 1268753 & $\begin{array}{l}\text { Peptide hormone } \\
\text { biosynthesis }\end{array}$ & $2.15 \mathrm{E}-06$ & $1.11 \mathrm{E}-03$ & $7.59 \mathrm{E}-03$ & $1.11 \mathrm{E}-03$ & 05 & $\begin{array}{l}\text { CGB3,TSHB,POMC,FSHB, } \\
\text { INHBA }\end{array}$ \\
\hline 1268754 & Glycoprotein hormones & $3.31 \mathrm{E}-05$ & $8.15 \mathrm{E}-03$ & $5.56 \mathrm{E}-02$ & $1.71 \mathrm{E}-02$ & 04 & CGB3,TSHB,FSHB,INHBA \\
\hline 1269559 & $\begin{array}{l}\text { Hormone ligand-binding } \\
\text { receptors }\end{array}$ & 4.72E-05 & $8.15 \mathrm{E}-03$ & $5.56 \mathrm{E}-02$ & $2.44 \mathrm{E}-02$ & 04 & $\begin{array}{l}\text { TSHB,FSHB,GPHA2,GNR } \\
\text { HR }\end{array}$ \\
\hline 1269328 & $\begin{array}{l}\text { Prolactin receptor } \\
\text { signaling }\end{array}$ & $1.16 \mathrm{E}-04$ & $1.38 \mathrm{E}-02$ & $9.41 \mathrm{E}-02$ & $5.98 \mathrm{E}-02$ & 04 & CSH1,CSHL1,GH1,GH2 \\
\hline 1268746 & $\begin{array}{l}\text { Peptide hormone } \\
\text { metabolism }\end{array}$ & $1.33 \mathrm{E}-04$ & $1.38 \mathrm{E}-02$ & $9.41 \mathrm{E}-02$ & $6.89 \mathrm{E}-02$ & 08 & $\begin{array}{l}\text { PCSK2,CGB3,TSHB,POMC } \\
\text {,FSHB,INHBA,GH1,PAX6 }\end{array}$ \\
\hline 1269327 & $\begin{array}{l}\text { Growth hormone receptor } \\
\text { signaling }\end{array}$ & 7.14E-04 & $6.16 \mathrm{E}-02$ & 4.21E-01 & $3.70 \mathrm{E}-01$ & 04 & CSH1,CSHL1,GH1,GH2 \\
\hline
\end{tabular}




\begin{tabular}{|c|c|c|c|c|c|c|c|}
\hline 1269545 & $\begin{array}{l}\text { Class A/1 (Rhodopsin-like } \\
\text { receptors) }\end{array}$ & $9.87 \mathrm{E}-04$ & $7.30 \mathrm{E}-02$ & 4.99E-01 & $5.11 \mathrm{E}-01$ & 14 & $\begin{array}{l}\text { GPR37L1,TSHB,POMC,FS } \\
\text { HB,GALR1,GPHA2,SSTR2 } \\
\text {,KISS1R,GNRHR,FFAR3,C } \\
\text { CK,CCKBR,GAL,P2RY2 }\end{array}$ \\
\hline 1270347 & $\begin{array}{l}\text { POU5F1 (OCT4), SOX2, } \\
\text { NANOG activate genes } \\
\text { related to proliferation }\end{array}$ & $1.15 \mathrm{E}-03$ & 7.42E-02 & $5.07 \mathrm{E}-01$ & $5.93 \mathrm{E}-01$ & 03 & SALL1,SOX2,DPPA4 \\
\hline 1269544 & GPCR ligand binding & $1.56 \mathrm{E}-03$ & $8.98 \mathrm{E}-02$ & $6.13 \mathrm{E}-01$ & $8.08 \mathrm{E}-01$ & 17 & $\begin{array}{l}\text { GPR37L1,TSHB,WNT5A,P } \\
\text { OMC,FSHB,IHH,GALR1,G } \\
\text { PHA2,SSTR2,GHRHR,KIS } \\
\text { S1R,GNRHR,FFAR3,CCK, } \\
\text { CCKBR,GAL,P2RY2 }\end{array}$ \\
\hline 1270340 & $\begin{array}{l}\text { Regulation of gene } \\
\text { expression in endocrine- } \\
\text { committed (NEUROG3+) } \\
\text { progenitor cells }\end{array}$ & $2.66 \mathrm{E}-03$ & $1.23 \mathrm{E}-01$ & $8.36 \mathrm{E}-01$ & $1.00 \mathrm{E}+00$ & 02 & NEUROD1,NKX2-2 \\
\hline
\end{tabular}

\section{GenMAPP}

\begin{tabular}{llcccccc}
\hline MAP00350 & Tyrosine metabolism & $1.32 \mathrm{E}-02$ & $2.47 \mathrm{E}-01$ & $8.99 \mathrm{E}-01$ & $2.78 \mathrm{E}-01$ & 03 & ALDH1A3,PNMT,AOX1 \\
MAP00150 & $\begin{array}{l}\text { Androgen and estrogen } \\
\text { metabolism }\end{array}$ & $3.18 \mathrm{E}-02$ & $2.47 \mathrm{E}-01$ & $8.99 \mathrm{E}-01$ & $6.67 \mathrm{E}-01$ & 02 & HSD11B1,HSD17B2 \\
MAP00380 & Tryptophan metabolism & $4.08 \mathrm{E}-02$ & $2.47 \mathrm{E}-01$ & $8.99 \mathrm{E}-01$ & $8.56 \mathrm{E}-01$ & 03 & AOX1,CYP4B1,TDO2 \\
MAP00910 & Nitrogen metabolism & $4.70 \mathrm{E}-02$ & $2.47 \mathrm{E}-01$ & $8.99 \mathrm{E}-01$ & $9.86 \mathrm{E}-01$ & 02 & CA8,CA12
\end{tabular}

\section{MSigDB C2 BIOCARTA (v6.0)}

\begin{tabular}{|c|c|c|c|c|c|c|c|}
\hline M5889 & $\begin{array}{l}\text { Ensemble of genes } \\
\text { encoding extracellular } \\
\text { matrix and extracellular } \\
\text { matrix-associated } \\
\text { proteins }\end{array}$ & $1.18 \mathrm{E}-09$ & $5.44 \mathrm{E}-08$ & $2.40 \mathrm{E}-07$ & $5.44 \mathrm{E}-08$ & 45 & $\begin{array}{l}\text { S100A14,VIT,S100A1,S100 } \\
\text { B,MFAP4,FBN2,EFEMP1,F } \\
\text { CN2,SBSPON,SPON1,FGF } \\
\text { 1,ITIH6,COL4A6,COL8A2, } \\
\text { FRAS1,SFRP2,COL13A1,I } \\
\text { TLN1,WNT5A,ADAM32,C } \\
\text { SH1,CSH2,CSHL1,SLIT1,S } \\
\text { LPI,IFNE,IHH,MEGF11,IN } \\
\text { HBA,LAMA1,ITIH2,GH1, } \\
\text { GH2,BMP7,COL21A1,ADA } \\
\text { MTS18,CHRDL1,CLEC9A, } \\
\text { NTF4,OGN,LEFTY2,RSPO } \\
\text { 3,MEGF6,CBLN2,LGI1 }\end{array}$ \\
\hline M5883 & $\begin{array}{l}\text { Genes encoding } \\
\text { secreted soluble factors }\end{array}$ & 2.79E-07 & $6.41 \mathrm{E}-06$ & 2.83E-05 & $1.28 \mathrm{E}-05$ & 21 & $\begin{array}{l}\text { S100A14,S100A1,S100B,F } \\
\text { GF1,SFRP2,WNT5A,CSH1, } \\
\text { CSH2,CSHL1,IFNE,IHH,M } \\
\text { EGF11,INHBA,GH1,GH2,B } \\
\text { MP7,CHRDL1,NTF4,LEFT } \\
\text { Y2,MEGF6,CBLN2 }\end{array}$ \\
\hline M5884 & $\begin{array}{l}\text { Ensemble of genes } \\
\text { encoding core } \\
\text { extracellular matrix } \\
\text { including ECM } \\
\text { glycoproteins, collagens } \\
\text { and proteoglycans }\end{array}$ & $1.42 \mathrm{E}-05$ & $2.18 \mathrm{E}-04$ & $9.63 \mathrm{E}-04$ & $6.54 \mathrm{E}-04$ & 16 & $\begin{array}{l}\text { VIT,MFAP4,FBN2,EFEMP } \\
\text { 1,SBSPON,SPON1,COL4A } \\
\text { 6,COL8A2,FRAS1,COL13A } \\
\text { 1,SLIT1,LAMA1,COL21A1 } \\
\text {,OGN,RSPO3,LGI1 }\end{array}$ \\
\hline
\end{tabular}




\begin{tabular}{|c|c|c|c|c|c|c|c|}
\hline M5885 & $\begin{array}{l}\text { Ensemble of genes } \\
\text { encoding ECM- } \\
\text { associated proteins } \\
\text { including ECM- } \\
\text { affilaited proteins, ECM } \\
\text { regulators and secreted } \\
\text { factors }\end{array}$ & $1.94 \mathrm{E}-05$ & $2.23 \mathrm{E}-04$ & $9.84 \mathrm{E}-04$ & 8.91E-04 & 29 & $\begin{array}{l}\text { S100A14,S100A1,S100B,F } \\
\text { CN2,FGF1,ITIH6,SFRP2,IT } \\
\text { LN1,WNT5A,ADAM32,CS } \\
\text { H1,CSH2,CSHL1,SLPI,IFN } \\
\text { E,IHH,MEGF11,INHBA,ITI } \\
\text { H2,GH1,GH2,BMP7, ADA } \\
\text { MTS18,CHRDL1,CLEC9A, } \\
\text { NTF4,LEFTY2,MEGF6,CB } \\
\text { LN2 }\end{array}$ \\
\hline M3008 & $\begin{array}{l}\text { Genes encoding } \\
\text { structural ECM } \\
\text { glycoproteins }\end{array}$ & 4.35E-04 & $4.00 \mathrm{E}-03$ & $1.77 \mathrm{E}-02$ & $2.00 \mathrm{E}-02$ & 11 & $\begin{array}{l}\text { VIT,MFAP4,FBN2,EFEMP } \\
\text { 1,SBSPON,SPON1,FRAS1, } \\
\text { SLIT1,LAMA1,RSPO3,LGI } \\
1\end{array}$ \\
\hline M4557 & $\begin{array}{l}\text { Biosynthesis of } \\
\text { neurotransmitters }\end{array}$ & $3.95 \mathrm{E}-03$ & $3.03 \mathrm{E}-02$ & $1.34 \mathrm{E}-01$ & $1.82 \mathrm{E}-01$ & 02 & PNMT,TPH1 \\
\hline M3005 & $\begin{array}{l}\text { Genes encoding } \\
\text { collagen proteins }\end{array}$ & $5.99 \mathrm{E}-03$ & $3.94 \mathrm{E}-02$ & $1.74 \mathrm{E}-01$ & $2.76 \mathrm{E}-01$ & 04 & $\begin{array}{l}\text { COL4A6,COL8A2,COL13A } \\
\text { 1,COL21A1 }\end{array}$ \\
\hline M16518 & $\begin{array}{l}\text { Wnt/Ca2+/cyclic GMP } \\
\text { signaling. }\end{array}$ & $4.30 \mathrm{E}-02$ & $2.47 \mathrm{E}-01$ & $1.00 \mathrm{E}+00$ & $1.00 \mathrm{E}+00$ & 02 & PDE6A,TF \\
\hline
\end{tabular}

\section{PantherDB}

\begin{tabular}{lllllllll}
\hline P02723 & $\begin{array}{l}\text { Adenine and } \\
\text { hypoxanthine salvage } \\
\text { pathway }\end{array}$ & $5.47 \mathrm{E}-03$ & $2.19 \mathrm{E}-01$ & $9.36 \mathrm{E}-01$ & $2.19 \mathrm{E}-01$ & 02 & AOX1,XDH \\
P04371 & $\begin{array}{l}\text { 5-Hydroxytryptamine } \\
\text { biosynthesis }\end{array}$ & $4.91 \mathrm{E}-02$ & $6.50 \mathrm{E}-01$ & $1.00 \mathrm{E}+00$ & $1.00 \mathrm{E}+00$ & 01 & TPH1
\end{tabular}

\section{Pathway Ontology}

\begin{tabular}{clcccccc}
\hline PW:0000365 & melanocortin system & $1.13 \mathrm{E}-02$ & $1.52 \mathrm{E}-01$ & $6.35 \mathrm{E}-01$ & $4.08 \mathrm{E}-01$ & 02 & PCSK2,POMC \\
PW:0000040 & $\begin{array}{l}\text { androgen and estrogen } \\
\text { metabolic }\end{array}$ & $1.37 \mathrm{E}-02$ & $1.52 \mathrm{E}-01$ & $6.35 \mathrm{E}-01$ & $4.94 \mathrm{E}-01$ & 02 & HSD11B1,UGT2A1 \\
PW:0000371 & calcium homeostasis & $1.69 \mathrm{E}-02$ & $1.52 \mathrm{E}-01$ & $6.35 \mathrm{E}-01$ & $6.09 \mathrm{E}-01$ & 01 & S100A1 \\
PW:0000138 & Vitamin B6 metabolic & $1.69 \mathrm{E}-02$ & $1.52 \mathrm{E}-01$ & $6.35 \mathrm{E}-01$ & $6.09 \mathrm{E}-01$ & 01 & AOX1 \\
PW:0000200 & $\begin{array}{l}\text { the planar cell polarity } \\
\text { Wntsignaling }\end{array}$ & $2.51 \mathrm{E}-02$ & $1.80 \mathrm{E}-01$ & $7.53 \mathrm{E}-01$ & $9.02 \mathrm{E}-01$ & 02 & CELSR1,VANGL2 \\
PW:0000190 & $\begin{array}{l}\text { porphyrin and } \\
\text { chlorophyll metabolic }\end{array}$ & $4.91 \mathrm{E}-02$ & $2.07 \mathrm{E}-01$ & $8.63 \mathrm{E}-01$ & $1.00 \mathrm{E}+00$ & 01 & UGT2A1
\end{tabular}

\section{SMPDB}

\begin{tabular}{|c|c|c|c|c|c|c|c|}
\hline SMP00063 & $\begin{array}{l}\text { Tryptophan } \\
\text { Metabolism }\end{array}$ & $2.59 \mathrm{E}-03$ & $1.16 \mathrm{E}-01$ & $5.13 \mathrm{E}-01$ & $1.19 \mathrm{E}-01$ & 03 & AOX1,TDO2,TPH1 \\
\hline SMP00220 & $\begin{array}{l}\text { Xanthine } \\
\text { Dehydrogenase } \\
\text { Deficiency } \\
\text { (Xanthinuria) }\end{array}$ & $1.69 \mathrm{E}-02$ & $1.16 \mathrm{E}-01$ & 5.13E-01 & $7.78 \mathrm{E}-01$ & 01 & $\mathrm{XDH}$ \\
\hline
\end{tabular}




$\begin{array}{clcccccc}\text { SMP00203 } & \begin{array}{l}\text { Molybdenium } \\ \text { Cofactor Deficiency }\end{array} & 1.69 \mathrm{E}-02 & 1.16 \mathrm{E}-01 & 5.13 \mathrm{E}-01 & 7.78 \mathrm{E}-01 & 01 & \text { XDH } \\ \text { SMP00246 } & \text { Pirenzepine Pathway } & 3.18 \mathrm{E}-02 & 1.16 \mathrm{E}-01 & 5.13 \mathrm{E}-01 & 1.00 \mathrm{E}+00 & 02 & \text { ATP4A,CCKBR } \\ \text { SMP00232 } & \text { Cimetidine Pathway } & 3.18 \mathrm{E}-02 & 1.16 \mathrm{E}-01 & 5.13 \mathrm{E}-01 & 1.00 \mathrm{E}+00 & 02 & \text { ATP4A,CCKBR } \\ \text { SMP00028 } & \text { Caffeine Metabolism } & 3.30 \mathrm{E}-02 & 1.16 \mathrm{E}-01 & 5.13 \mathrm{E}-01 & 1.00 \mathrm{E}+00 & 01 & \text { XDH } \\ \text { SMP00233 } & \text { Nizatidine Pathway } & 3.53 \mathrm{E}-02 & 1.16 \mathrm{E}-01 & 5.13 \mathrm{E}-01 & 1.00 \mathrm{E}+00 & 02 & \text { ATP4A,CCKBR } \\ \text { SMP00226 } & \text { Omeprazole Pathway } & 3.53 \mathrm{E}-02 & 1.16 \mathrm{E}-01 & 5.13 \mathrm{E}-01 & 1.00 \mathrm{E}+00 & 02 & \text { ATP4A,CCKBR } \\ \text { SMP00231 } & \text { Famotidine Pathway } & 3.53 \mathrm{E}-02 & 1.16 \mathrm{E}-01 & 5.13 \mathrm{E}-01 & 1.00 \mathrm{E}+00 & 02 & \text { ATP4A,CCKBR } \\ \text { SMP00227 } & \text { Lansoprazole Pathway } & 3.53 \mathrm{E}-02 & 1.16 \mathrm{E}-01 & 5.13 \mathrm{E}-01 & 1.00 \mathrm{E}+00 & 02 & \text { ATP4A,CCKBR }\end{array}$

Table 3 The enriched pathway terms of the down-regulated differentially expressed genes BIOCYC

\begin{tabular}{|c|c|c|c|c|c|c|c|}
\hline $\begin{array}{l}\text { Pathway } \\
\text { ID }\end{array}$ & Pathway Name & P-value & $\begin{array}{l}\text { FDR } \\
\text { B\&H }\end{array}$ & FDR B\&Y & Bonferroni & $\begin{array}{l}\text { Gene } \\
\text { Count }\end{array}$ & Gene \\
\hline 545291 & vitamin D3 biosynthesis & $2.55 \mathrm{E}-02$ & $2.81 \mathrm{E}-01$ & $8.48 \mathrm{E}-01$ & $2.81 \mathrm{E}-01$ & 01 & CYP27B1 \\
\hline \multicolumn{8}{|c|}{ KEGG } \\
\hline 83054 & Cell cycle & $1.09 \mathrm{E}-03$ & $1.56 \mathrm{E}-01$ & $8.62 \mathrm{E}-01$ & $1.56 \mathrm{E}-01$ & 07 & $\begin{array}{l}\text { TTK,CDC45,PLK1,PTT } \\
\text { G2,BUB1,CCNA1,CCN } \\
\text { B2 }\end{array}$ \\
\hline 169306 & Pancreatic secretion & $7.88 \mathrm{E}-03$ & $5.44 \mathrm{E}-01$ & $1.00 \mathrm{E}+00$ & $1.00 \mathrm{E}+00$ & 05 & $\begin{array}{l}\text { RYR2,ATP2A3,ATP2B } \\
\text { 2,PLA2G2D,CELA3A }\end{array}$ \\
\hline 83055 & p53 signaling pathway & 1.19E-02 & $5.44 \mathrm{E}-01$ & $1.00 \mathrm{E}+00$ & $1.00 \mathrm{E}+00$ & 04 & $\begin{array}{l}\text { GTSE1,CCNG2,ZMAT } \\
3, \mathrm{CCNB} 2\end{array}$ \\
\hline 413367 & Lactosylceramide biosynthesis & $2.55 \mathrm{E}-02$ & $5.44 \mathrm{E}-01$ & $1.00 \mathrm{E}+00$ & $1.00 \mathrm{E}+00$ & 01 & B4GALT6 \\
\hline 413391 & Cholecalciferol biosynthesis & $2.55 \mathrm{E}-02$ & $5.44 \mathrm{E}-01$ & $1.00 \mathrm{E}+00$ & $1.00 \mathrm{E}+00$ & 01 & CYP27B1 \\
\hline 83050 & Calcium signaling pathway & $3.01 \mathrm{E}-02$ & $5.44 \mathrm{E}-01$ & $1.00 \mathrm{E}+00$ & $1.00 \mathrm{E}+00$ & 06 & $\begin{array}{l}\text { CHRNA7,RYR2,ATP2 } \\
\text { A3,ATP2B2,PLCD4,GR } \\
\text { M5 }\end{array}$ \\
\hline 377262 & Fanconianemia pathway & $3.36 \mathrm{E}-02$ & $5.44 \mathrm{E}-01$ & $1.00 \mathrm{E}+00$ & $1.00 \mathrm{E}+00$ & 03 & $\begin{array}{l}\text { FANCA,FANCD2,RAD } \\
51\end{array}$ \\
\hline 119304 & $\begin{array}{l}\text { Progesterone-mediated oocyte } \\
\text { maturation }\end{array}$ & $3.51 \mathrm{E}-02$ & $5.44 \mathrm{E}-01$ & $1.00 \mathrm{E}+00$ & $1.00 \mathrm{E}+00$ & 04 & $\begin{array}{l}\text { PLK1,BUB1,CCNA1,C } \\
\text { CNB2 }\end{array}$ \\
\hline 199556 & Vitamin digestion and absorption & $3.77 \mathrm{E}-02$ & $5.44 \mathrm{E}-01$ & $1.00 \mathrm{E}+00$ & $1.00 \mathrm{E}+00$ & 02 & SLC19A1,SLC52A3 \\
\hline 413396 & $\begin{array}{l}\text { Steroid hormone biosynthesis, } \\
\text { cholesterol => prognenolone => } \\
\text { progesterone }\end{array}$ & $3.81 \mathrm{E}-02$ & $5.44 \mathrm{E}-01$ & $1.00 \mathrm{E}+00$ & $1.00 \mathrm{E}+00$ & 01 & HSD3B2 \\
\hline 83096 & Maturity onset diabetes of the & 4.36E-02 & $5.67 \mathrm{E}-01$ & $1.00 \mathrm{E}+00$ & $1.00 \mathrm{E}+00$ & 02 & PKLR,PAX4 \\
\hline
\end{tabular}




\begin{tabular}{|c|c|c|c|c|c|c|c|}
\hline \multicolumn{8}{|c|}{ Pathway Interaction Database } \\
\hline 138080 & Aurora B signaling & $1.16 \mathrm{E}-09$ & $4.30 \mathrm{E}-08$ & $1.81 \mathrm{E}-07$ & $4.30 \mathrm{E}-08$ & 09 & $\begin{array}{l}\text { CENPA,KLHL13,KIF2 } \\
\text { 3,BIRC5,RACGAP1,N } \\
\text { CAPG,BUB1,STMN1,K } \\
\text { IF20A }\end{array}$ \\
\hline 137935 & $\begin{array}{l}\text { FOXM1 transcription factor } \\
\text { network }\end{array}$ & $6.86 \mathrm{E}-06$ & $1.27 \mathrm{E}-04$ & 5.33E-04 & $2.54 \mathrm{E}-04$ & 06 & $\begin{array}{l}\text { CENPA,PLK1,FOXM1, } \\
\text { BIRC5,NEK2,CCNB2 }\end{array}$ \\
\hline 138007 & PLK1 signaling events & $1.93 \mathrm{E}-05$ & $2.38 \mathrm{E}-04$ & $1.00 \mathrm{E}-03$ & $7.15 \mathrm{E}-04$ & 06 & $\begin{array}{l}\text { PLK1,SPC24,TPX2,BU } \\
\text { B1,CENPU,KIF20A }\end{array}$ \\
\hline 137925 & Aurora A signaling & $6.31 \mathrm{E}-04$ & $5.83 \mathrm{E}-03$ & $2.45 \mathrm{E}-02$ & $2.33 \mathrm{E}-02$ & 04 & $\begin{array}{l}\text { CENPA,BIRC5,TPX2,D } \\
\text { LGAP5 }\end{array}$ \\
\hline 137959 & BARD1 signaling events & $5.96 \mathrm{E}-03$ & 4.41E-02 & $1.85 \mathrm{E}-01$ & $2.20 \mathrm{E}-01$ & 03 & $\begin{array}{l}\text { FANCA,FANCD2,RAD } \\
51\end{array}$ \\
\hline
\end{tabular}

REACTOME

\begin{tabular}{|c|c|c|c|c|c|c|c|}
\hline 1269820 & Mitotic Prometaphase & $1.04 \mathrm{E}-11$ & $5.07 \mathrm{E}-09$ & $3.43 \mathrm{E}-08$ & $5.07 \mathrm{E}-09$ & 15 & $\begin{array}{l}\text { KIF18A,CENPA,SPC25 } \\
\text {,CENPM,PLK1,BIRC5, } \\
\text { SPC24,CENPH,CENPI, } \\
\text { CDCA5,NUF2,NCAPG, } \\
\text { BUB1,CENPU,CCNB2 }\end{array}$ \\
\hline 1269821 & $\begin{array}{l}\text { Resolution of Sister Chromatid } \\
\text { Cohesion }\end{array}$ & $4.85 \mathrm{E}-11$ & $1.18 \mathrm{E}-08$ & 7.97E-08 & $2.36 \mathrm{E}-08$ & 14 & $\begin{array}{l}\text { KIF18A,CENPA,SPC25 } \\
\text {,CENPM,PLK1,BIRC5, } \\
\text { SPC24,CENPH,CENPI, } \\
\text { CDCA5,NUF2,BUB1,C } \\
\text { ENPU,CCNB2 }\end{array}$ \\
\hline 1269741 & Cell Cycle & $2.94 \mathrm{E}-10$ & $4.76 \mathrm{E}-08$ & $3.22 \mathrm{E}-07$ & $1.43 \mathrm{E}-07$ & 30 & $\begin{array}{l}\text { KIF18A,CENPA,HJUR } \\
\text { P,SPC25,MND1,HMM } \\
\text { R,CDC45,CENPM,PLK } \\
\text { 1,FOXM1,KIF23,GTSE } \\
\text { 1,BIRC5,SPC24,CENP } \\
\text { H,CENPI,TPX2,CDCA } \\
\text { 5,HAUS6,NUF2,NEK2, } \\
\text { NCAPG,BUB1,CCNA1, } \\
\text { RAD51,CENPU,RFC3, } \\
\text { KIF20A,CCNB2,TOP2 } \\
\text { A }\end{array}$ \\
\hline 1269763 & Cell Cycle, Mitotic & $4.25 \mathrm{E}-10$ & $5.16 \mathrm{E}-08$ & $3.49 \mathrm{E}-07$ & 2.07E-07 & 27 & $\begin{array}{l}\text { KIF18A,CENPA,SPC25 } \\
\text {,HMMR,CDC45,CENP } \\
\text { M,PLK1,FOXM1,KIF2 } \\
\text { 3,GTSE1,BIRC5,SPC24 } \\
\text {,CENPH,CENPI,TPX2, } \\
\text { CDCA5,HAUS6,NUF2, } \\
\text { NEK2,NCAPG,BUB1,C } \\
\text { CNA1,CENPU,RFC3,K } \\
\text { IF20A,CCNB2,TOP2A }\end{array}$ \\
\hline 1269519 & RHO GTPases Activate Formins & $3.21 \mathrm{E}-08$ & $3.12 \mathrm{E}-06$ & $2.11 \mathrm{E}-05$ & $1.56 \mathrm{E}-05$ & 12 & $\begin{array}{l}\text { KIF18A,CENPA,SPC25 } \\
\text {,CENPM,PLK1,BIRC5, } \\
\text { SPC24,CENPH,CENPI, }\end{array}$ \\
\hline
\end{tabular}


NUF2,BUB1,CENPU

\begin{tabular}{|c|c|c|c|c|c|c|c|}
\hline 1269378 & Kinesins & $6.18 \mathrm{E}-08$ & 5.01E-06 & $3.39 \mathrm{E}-05$ & $3.00 \mathrm{E}-05$ & 08 & $\begin{array}{l}\text { KIF18A,KIF23,RACGA } \\
\text { P1,KIF21B,KIF26A,KI } \\
\text { F15,KIF11,KIF20A }\end{array}$ \\
\hline 1269826 & Separation of Sister Chromatids & $2.79 \mathrm{E}-07$ & $1.94 \mathrm{E}-05$ & $1.31 \mathrm{E}-04$ & $1.36 \mathrm{E}-04$ & 13 & $\begin{array}{l}\text { KIF18A,CENPA,SPC25 } \\
\text {,CENPM,PLK1,BIRC5, } \\
\text { SPC24,CENPH,CENPI, } \\
\text { CDCA5,NUF2,BUB1,C } \\
\text { ENPU }\end{array}$ \\
\hline 1269810 & M Phase & 5.07E-07 & $2.98 \mathrm{E}-05$ & 2.01E-04 & $2.46 \mathrm{E}-04$ & 17 & $\begin{array}{l}\text { KIF18A,CENPA,SPC25 } \\
\text {,CENPM,PLK1,KIF23, } \\
\text { BIRC5,SPC24,CENPH, } \\
\text { CENPI,CDCA5,NUF2, } \\
\text { NCAPG,BUB1,CENPU, } \\
\text { KIF20A,CCNB2 }\end{array}$ \\
\hline 1269825 & Mitotic Anaphase & $5.75 \mathrm{E}-07$ & $2.98 \mathrm{E}-05$ & 2.01E-04 & 2.79E-04 & 13 & $\begin{array}{l}\text { KIF18A,CENPA,SPC25 } \\
\text {,CENPM,PLK1,BIRC5, } \\
\text { SPC24,CENPH,CENPI, } \\
\text { CDCA5,NUF2,BUB1,C } \\
\text { ENPU }\end{array}$ \\
\hline 1269823 & Mitotic Metaphase and Anaphase & $6.12 \mathrm{E}-07$ & $2.98 \mathrm{E}-05$ & $2.01 \mathrm{E}-04$ & $2.98 \mathrm{E}-04$ & 13 & $\begin{array}{l}\text { KIF18A,CENPA,SPC25 } \\
\text {,CENPM,PLK1,BIRC5, } \\
\text { SPC24,CENPH,CENPI, } \\
\text { CDCA5,NUF2,BUB1,C } \\
\text { ENPU }\end{array}$ \\
\hline 1269799 & G2/M Transition & $6.15 \mathrm{E}-04$ & $1.42 \mathrm{E}-02$ & $9.62 \mathrm{E}-02$ & 2.99E-01 & 09 & $\begin{array}{l}\text { HMMR,PLK1,FOXM1, } \\
\text { GTSE1,TPX2,HAUS6, } \\
\text { NEK2,CCNA1,CCNB2 }\end{array}$ \\
\hline
\end{tabular}

\section{MSigDB C2 BIOCARTA (v6.0)}

\begin{tabular}{llcccccc}
\hline M17370 & $\begin{array}{l}\text { Role of Ran in mitotic spindle } \\
\text { regulation }\end{array}$ & $2.34 \mathrm{E}-04$ & $8.19 \mathrm{E}-03$ & $3.40 \mathrm{E}-02$ & $8.19 \mathrm{E}-03$ & 03 & RCC1,TPX2,KIF15 \\
M16334 & $\begin{array}{l}\text { Eph Kinases and ephrins support } \\
\text { platelet aggregation }\end{array}$ & $6.90 \mathrm{E}-03$ & $1.21 \mathrm{E}-01$ & $5.01 \mathrm{E}-01$ & $2.42 \mathrm{E}-01$ & 02 & L1CAM,EPHB1 \\
M9703 & $\begin{array}{l}\text { Role of BRCA1, BRCA2 and } \\
\text { ATR in Cancer Susceptibility }\end{array}$ & $2.94 \mathrm{E}-02$ & $3.43 \mathrm{E}-01$ & $1.00 \mathrm{E}+00$ & $1.00 \mathrm{E}+00$ & 02 & FANCD2,RAD51 \\
\hline
\end{tabular}

\section{PantherDB}

\begin{tabular}{|c|c|c|c|c|c|c|c|}
\hline P00031 & $\begin{array}{l}\text { Inflammation mediated by } \\
\text { chemokine and cytokine signaling } \\
\text { pathway }\end{array}$ & $1.17 \mathrm{E}-02$ & $3.74 \mathrm{E}-01$ & $1.00 \mathrm{E}+00$ & $3.74 \mathrm{E}-01$ & 07 & $\begin{array}{l}\text { PF4,VWF,CCL3L3,CA } \\
\text { SK,CXCR2,CXCR3,PL } \\
\text { CD4 }\end{array}$ \\
\hline
\end{tabular}

\section{Pathway Ontology}

\begin{tabular}{llllllll}
\hline PW:0000194 & O-Glycans biosynthetic & $1.30 \mathrm{E}-02$ & $5.02 \mathrm{E}-02$ & $1.95 \mathrm{E}-01$ & $3.52 \mathrm{E}-01$ & 01 & B4GALT6 \\
PW:0000164 & ganglioside biosynthetic & $1.30 \mathrm{E}-02$ & $5.02 \mathrm{E}-02$ & $1.95 \mathrm{E}-01$ & $3.52 \mathrm{E}-01$ & 01 & B4GALT6 \\
& & & & & & & \\
PW:0000196 & globoside metabolic & $1.30 \mathrm{E}-02$ & $5.02 \mathrm{E}-02$ & $1.95 \mathrm{E}-01$ & $3.52 \mathrm{E}-01$ & 01 & B4GALT6
\end{tabular}




\begin{tabular}{|c|c|c|c|c|c|c|c|}
\hline PW:0000197 & glycosphingolipid metabolic & $1.30 \mathrm{E}-02$ & $5.02 \mathrm{E}-02$ & $1.95 \mathrm{E}-01$ & $3.52 \mathrm{E}-01$ & 01 & B4GALT6 \\
\hline PW:0000161 & $\begin{array}{l}\text { glycosylphosphatidylinositol } \\
\text { anchor biosynthetic }\end{array}$ & $1.30 \mathrm{E}-02$ & $5.02 \mathrm{E}-02$ & $1.95 \mathrm{E}-01$ & $3.52 \mathrm{E}-01$ & 01 & B4GALT6 \\
\hline PW:0000162 & $\begin{array}{l}\text { glycosphingolipid } \\
\text { biosynthetic - lactoseries }\end{array}$ & $1.30 \mathrm{E}-02$ & 5.02E-02 & $1.95 \mathrm{E}-01$ & $3.52 \mathrm{E}-01$ & 01 & B4GALT6 \\
\hline PW:0000163 & $\begin{array}{l}\text { glycosphingolipid } \\
\text { biosynthetic - neolactoseries }\end{array}$ & $1.30 \mathrm{E}-02$ & 5.02E-02 & $1.95 \mathrm{E}-01$ & $3.52 \mathrm{E}-01$ & 01 & B4GALT6 \\
\hline PW:0000192 & N-Glycans biosynthetic & $2.55 \mathrm{E}-02$ & 8.04E-02 & $3.13 \mathrm{E}-01$ & $6.90 \mathrm{E}-01$ & 01 & B4GALT6 \\
\hline PW:0000017 & Huntington disease & $2.68 \mathrm{E}-02$ & 8.04E-02 & 3.13E-01 & 7.24E-01 & 02 & CLTC,BDNF \\
\hline
\end{tabular}

\section{SMPDB}

\begin{tabular}{clcccccc}
\hline SMP00266 & Eptifibatide Pathway & $9.96 \mathrm{E}-03$ & $9.96 \mathrm{E}-03$ & $1.05 \mathrm{E}-01$ & $4.94 \mathrm{E}-01$ & 02 & RYR2,PLCD4 \\
SMP00261 & Ticlopidine Pathway & $9.96 \mathrm{E}-03$ & $9.96 \mathrm{E}-03$ & $1.05 \mathrm{E}-01$ & $4.94 \mathrm{E}-01$ & 02 & RYR2,PLCD4 \\
SMP00265 & Abciximab Pathway & $9.96 \mathrm{E}-03$ & $9.96 \mathrm{E}-03$ & $1.05 \mathrm{E}-01$ & $4.94 \mathrm{E}-01$ & 02 & RYR2,PLCD4 \\
SMP00264 & Dipyridamole Pathway & $9.96 \mathrm{E}-03$ & $9.96 \mathrm{E}-03$ & $1.05 \mathrm{E}-01$ & $4.94 \mathrm{E}-01$ & 02 & RYR2,PLCD4 \\
SMP00263 & Cilostazol Pathway & $9.96 \mathrm{E}-03$ & $9.96 \mathrm{E}-03$ & $1.05 \mathrm{E}-01$ & $4.94 \mathrm{E}-01$ & 02 & RYR2,PLCD4 \\
SMP00267 & Tirofiban Pathway & $9.96 \mathrm{E}-03$ & $9.96 \mathrm{E}-03$ & $1.05 \mathrm{E}-01$ & $4.94 \mathrm{E}-01$ & 02 & RYR2,PLCD4 \\
SMP00336 & Vitamin A Deficiency & $1.30 \mathrm{E}-02$ & $1.30 \mathrm{E}-02$ & $1.17 \mathrm{E}-01$ & $5.54 \mathrm{E}-01$ & 01 & BCO1 \\
SMP00260 & Clopidogrel Pathway & $2.19 \mathrm{E}-02$ & $2.19 \mathrm{E}-02$ & $1.73 \mathrm{E}-01$ & $8.17 \mathrm{E}-01$ & 02 & RYR2,PLCD4 \\
SMP00367 & Carvedilol Pathway & $2.94 \mathrm{E}-02$ & $2.94 \mathrm{E}-02$ & $1.85 \mathrm{E}-01$ & $8.75 \mathrm{E}-01$ & 02 & RYR2,PLCD4 \\
SMP00368 & Labetalol Pathway & $2.94 \mathrm{E}-02$ & $2.94 \mathrm{E}-02$ & $1.85 \mathrm{E}-01$ & $8.75 \mathrm{E}-01$ & 02 & RYR2,PLCD4
\end{tabular}

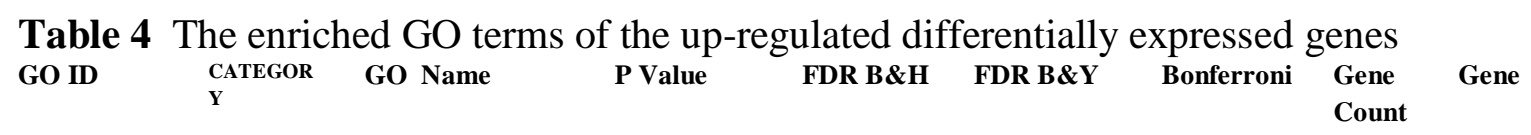

\begin{tabular}{|c|c|c|c|c|c|c|c|c|}
\hline GO:0090596 & BP & $\begin{array}{l}\text { sensory organ } \\
\text { morphogenesis }\end{array}$ & $1.00 \mathrm{E}-12$ & $4.43 \mathrm{E}-09$ & $3.97 \mathrm{E}-08$ & $4.43 \mathrm{E}-09$ & 28 & $\begin{array}{l}\text { EYA4,FBN2,E } \\
\text { FEMP1,SALL1, } \\
\text { CLIC5,TMIE,F } \\
\text { GFR3,FGFR2, } \\
\text { ALDH1A3,CL } \\
\text { RN1,PRRX2,C } \\
\text { OL8A2,SDK2, } \\
\text { WNT5A,CELS } \\
\text { R1,IHH,MEGF } \\
\text { 11,SOX2,SOX9 } \\
\text {,GAS1,BMP7,F } \\
\text { OXL2,STRA6, } \\
\text { CALB1,ROR2, } \\
\text { RBP4,VANGL } \\
\text { 2,PAX6 }\end{array}$ \\
\hline GO:0048562 & BP & embryonic organ & $1.04 \mathrm{E}-11$ & 2.29E-08 & $2.06 \mathrm{E}-07$ & $4.58 \mathrm{E}-08$ & 28 & $\begin{array}{l}\text { EYA4,GRHL2, } \\
\text { OVOL2,FBN2, }\end{array}$ \\
\hline
\end{tabular}


morphogenesis

embryonic organ development

sensory organ development

embryonic morphogenesis
$6.48 \mathrm{E}-11$

7.17E-08

6.43E-07

2.87E-07

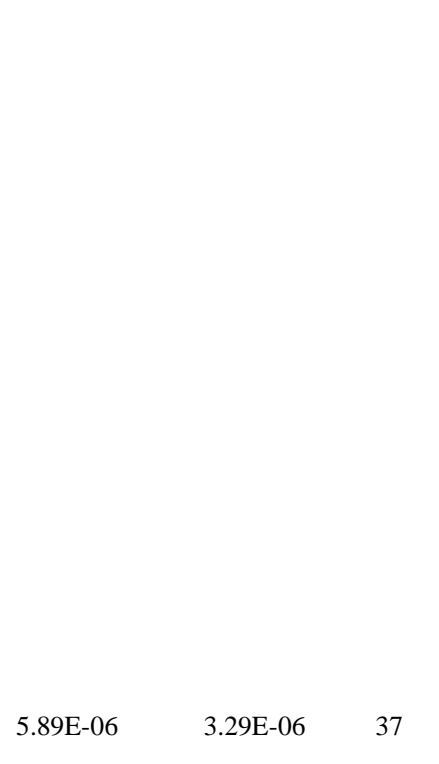

6.57E-07
EFEMP1,SALL 1,CLIC5,TMIE, FGFR2,ALDH1 A3,CLRN1,PR RX2,WNT5A,C ELSR1,IHH,RN F207,SOX2,SO X9,GAS1,BMP 7,NEUROD1,F OXL2,TBXT,S TRA6,ROR2,R BP4,VANGL2, PAX6

EYA4,GRHL2, OVOL2,FBN2, EFEMP1,SALL 1,CLIC5,TMIE, FGFR2,ALDH1 A3,CLRN1,PR RX2,WNT5A,C ELSR1,IHH,RN F207,SOX2,SO X9,PLAC1,GA S1,BMP7,NEU ROD1,FOXL2, NRK,TBXT,ST RA6,KRT19,R OR2,RBP4,RU NX1, VANGL2, RSPO3,PAX6

EYA4,PDE6A, GRHL2,FBN2, EFEMP1,SALL 1,CLIC5,TMIE, FGFR3,FGFR2, ALDH1 A3,CL RN1,PRRX2,C OL8A2,SDK2, WNT5A,CELS R1,IHH,PRDM 16,MEGF11,SO X2,SOX9,INH

BA,GAS1,LAM A1,BMP7,NEU ROD1,FOXL2, ADAMTS18,C HRDL1,STRA6 ,CALB1,NTF4, ROR2,RBP4,V ANGL2,PAX6

EYA4,EYA2,G RHL2,OVOL2, FBN2,EFEMP1 ,SALL1,CLIC5, TMIE,AMOT,F GFR2,ALDH1 A3,CLRN1,PR RX2,FRAS1,SF 


\begin{tabular}{|c|c|c|c|c|c|c|c|c|}
\hline & & & & & & & & $\begin{array}{l}\text { RP2,WNT5A,T } \\
\text { RIM71,IRX1,C } \\
\text { ELSR1,IHH,RN } \\
\text { F207,SOX2,SO } \\
\text { X9,INHBA,GA } \\
\text { S1,BMP7,NEU } \\
\text { ROD1,FOXL2, } \\
\text { TBXT,STRA6, } \\
\text { ROR2,RBP4,V } \\
\text { ANGL2,RSPO3 } \\
\text {,PAX6,IRX2 }\end{array}$ \\
\hline GO:0009887 & $\mathrm{BP}$ & $\begin{array}{l}\text { animal organ } \\
\text { morphogenesis }\end{array}$ & 3.37E-09 & $2.28 \mathrm{E}-06$ & $2.05 \mathrm{E}-05$ & $1.49 \mathrm{E}-05$ & 50 & $\begin{array}{l}\text { EYA4,GRHL2, } \\
\text { PGR,OVOL2,F } \\
\text { BN2,EFEMP1, } \\
\text { SALL1,CLIC5, } \\
\text { TMIE,FGF1,FG } \\
\text { FR3,FGFR2,FL } \\
\text { RT2,ALDH1A3 } \\
\text {,CLRN1,PRRX } \\
\text { 2,COL8A2,FR } \\
\text { AS1,SDK2,SFR } \\
\text { P2,COL13A1, } \\
\text { WNT5A,ID4,IR } \\
\text { X1,CELSR1,SL } \\
\text { IT1,IHH,RNF2 } \\
\text { 07,MEGF11,SO } \\
\text { X2,SOX9,INH } \\
\text { BA,GAS1,LAM } \\
\text { A1,BMP7,NEU } \\
\text { ROD1,FOXL2, } \\
\text { NGFR,TBXT,S } \\
\text { TRA6,CALB1, } \\
\text { NTF4,ROR2,R } \\
\text { BP4,VANGL2, } \\
\text { RSPO3,TGFBR } \\
\text { 3,PAX6,LY6H, } \\
\text { IRX2 }\end{array}$ \\
\hline GO:0048592 & $\mathrm{BP}$ & $\begin{array}{l}\text { eye } \\
\text { morphogenesis }\end{array}$ & 3.61E-09 & $2.28 \mathrm{E}-06$ & $2.05 \mathrm{E}-05$ & $1.60 \mathrm{E}-05$ & 18 & $\begin{array}{l}\text { FBN2,EFEMP1 } \\
\text {,FGFR3,ALDH } \\
\text { 1A3,COL8A2,S } \\
\text { DK2,WNT5A,I } \\
\text { HH,MEGF11,S } \\
\text { OX2,SOX9,GA } \\
\text { S1,BMP7,FOX } \\
\text { L2,STRA6,CA } \\
\text { LB1,RBP4,PA } \\
\text { X6 }\end{array}$ \\
\hline GO:0009790 & $\mathrm{BP}$ & $\begin{array}{l}\text { embryo } \\
\text { development }\end{array}$ & $5.07 \mathrm{E}-09$ & $2.80 \mathrm{E}-06$ & $2.51 \mathrm{E}-05$ & $2.24 \mathrm{E}-05$ & 50 & $\begin{array}{l}\text { LRAT,ERBB4, } \\
\text { EYA4,EYA2,G } \\
\text { RHL2,OVOL2, } \\
\text { FBN2,EFEMP1 } \\
\text {,SALL1,CLIC5, } \\
\text { TMIE,AMOT,F } \\
\text { GFR2,ALDH1 } \\
\text { A3,HSD17B2,C } \\
\text { LRN1,PRRX2, } \\
\text { FRAS1,SFRP2, } \\
\text { WNT5A,TRIM }\end{array}$ \\
\hline
\end{tabular}




\begin{tabular}{|c|c|c|c|c|c|c|c|c|}
\hline GO:0060429 & $\mathrm{BP}$ & $\begin{array}{l}\text { epithelium } \\
\text { development }\end{array}$ & 7.84E-09 & $3.85 \mathrm{E}-06$ & $3.45 \mathrm{E}-05$ & $3.47 \mathrm{E}-05$ & 54 & $\begin{array}{l}\text { ERBB4,DACT2 } \\
\text {,PTER,GRHL2, } \\
\text { PGR,OVOL2,S } \\
\text { ALL1,CLIC5,N } \\
\text { R0B1,FGF1,FG } \\
\text { FR3,FGFR2,AL } \\
\text { DH1A3,CLRN1 } \\
\text {,DEUP1,FRAS1 } \\
\text {,SFRP2,WNT5 } \\
\text { A,AGR2,ID4,X } \\
\text { DH,TRIM71,IR } \\
\text { X1,CELSR1,IH } \\
\text { H,RNF207,SO } \\
\text { X2,SOX9,PRSS } \\
\text { 8,INHBA,GAS } \\
\text { 1,LAMA1,BMP } \\
\text { 7,NEUROD1,F } \\
\text { OXL2,NGFR,T } \\
\text { BXT,NKX2- } \\
\text { 2,KAZN,STRA } \\
\text { 6,KRT14,CAL } \\
\text { B1,NTF4,ROR2 } \\
\text {,OCA2,RUNX1 } \\
\text {,VANGL2,RSP } \\
\text { O3,TGFBR3,Z } \\
\text { NF750,GAL,PA } \\
\text { X6,IRX2,SMA } \\
\text { D9 }\end{array}$ \\
\hline GO:0072089 & $\mathrm{BP}$ & $\begin{array}{l}\text { stem cell } \\
\text { proliferation }\end{array}$ & $1.16 \mathrm{E}-08$ & $5.13 \mathrm{E}-06$ & $4.60 \mathrm{E}-05$ & $5.13 \mathrm{E}-05$ & 17 & $\begin{array}{l}\text { OVOL2,FGFR2 } \\
\text {,PRRX2,SFRP2 } \\
\text {,WNT5A,ID4,T } \\
\text { RIM71,CSHL1, } \\
\text { IHH,SOX2,SO } \\
\text { X5,SOX9,GAS } \\
\text { 1,BMP7,KCNA } \\
\text { 1,RUNX1,PAX } \\
6\end{array}$ \\
\hline GO:0031012 & $\mathrm{CC}$ & $\begin{array}{l}\text { extracellular } \\
\text { matrix }\end{array}$ & $1.21 \mathrm{E}-08$ & $4.58 \mathrm{E}-06$ & $2.98 \mathrm{E}-05$ & $4.58 \mathrm{E}-06$ & 28 & $\begin{array}{l}\text { VIT,MFAP4,FB } \\
\text { N2,EFEMP1,S } \\
\text { BSPON,SPON1 } \\
\text { „FGF1,FGFR2, } \\
\text { FLRT2,COL4A } \\
\text { 6,COL8A2,FR }\end{array}$ \\
\hline
\end{tabular}




\begin{tabular}{|c|c|c|c|c|c|c|c|c|}
\hline & & & & & & & & $\begin{array}{l}\text { AS1,SFRP2,W } \\
\text { NT5A,SLIT1,S } \\
\text { LPI,IHH,LAM } \\
\text { A1,BMP7,COL } \\
\text { 21A1,ADAMT } \\
\text { S18,CPXM2,L } \\
\text { AD1,RUNX1,O } \\
\text { GN,TF,LEFTY } \\
\text { 2,TGFBR3 }\end{array}$ \\
\hline GO:0005615 & $\mathrm{CC}$ & extracellular space & $1.37 \mathrm{E}-07$ & $2.60 \mathrm{E}-05$ & $1.69 \mathrm{E}-04$ & 5.19E-05 & 55 & $\begin{array}{l}\text { PCSK2,CRISP2 } \\
\text {,SCRG1,TAC4, } \\
\text { F5,S100B,EFE } \\
\text { MP1,FCN2,SP } \\
\text { ON1,FGF1,FLR } \\
\text { T2,SPINK8,SF } \\
\text { RP2,PI16,WNT } \\
\text { 5A,AGR2,POM } \\
\text { C,PON3,XDH, } \\
\text { APOD,CARTP } \\
\text { T,SCGB1D2,S } \\
\text { CGB1D1,CSHL } \\
\text { 1,ASIP,SLIT1,S } \\
\text { LPI,C2orf40,IF } \\
\text { NE,IHH,CTRB } \\
\text { 1,ATP4A,IL6R, } \\
\text { PRSS8,INHBA, } \\
\text { LAMA1,DLK1, } \\
\text { ITIH2,SMR3B, } \\
\text { GH1,BMP7,KL } \\
\text { K11,CPXM2,R } \\
\text { BP4,KLK12,FA } \\
\text { M3B,OGN,TF, } \\
\text { CCK,LEFTY2, } \\
\text { TGFBR3,GAL, } \\
\text { DMKN,CBLN2 } \\
\text { „LGI1 }\end{array}$ \\
\hline GO:0044420 & $\mathrm{CC}$ & $\begin{array}{l}\text { extracellular } \\
\text { matrix component }\end{array}$ & $2.57 \mathrm{E}-04$ & $3.26 \mathrm{E}-02$ & $2.13 \mathrm{E}-01$ & $9.78 \mathrm{E}-02$ & 10 & $\begin{array}{l}\text { MFAP4,FBN2, } \\
\text { EFEMP1,COL4 } \\
\text { A6,COL8A2,F } \\
\text { RAS1,LAMA1, } \\
\text { LAD1,RUNX1, } \\
\text { TF }\end{array}$ \\
\hline GO:0005604 & $\mathrm{CC}$ & $\begin{array}{l}\text { basement } \\
\text { membrane }\end{array}$ & 8.42E-04 & $8.00 \mathrm{E}-02$ & $5.21 \mathrm{E}-01$ & $3.20 \mathrm{E}-01$ & 08 & $\begin{array}{l}\text { EFEMP1,COL4 } \\
\text { A6,COL8A2,F } \\
\text { RAS1,LAMA1, } \\
\text { LAD1,RUNX1, } \\
\text { TF }\end{array}$ \\
\hline GO:0043204 & $\mathrm{CC}$ & perikaryon & $1.16 \mathrm{E}-03$ & 8.84E-02 & $5.76 \mathrm{E}-01$ & 4.42E-01 & 09 & $\begin{array}{l}\text { PCSK2,PGR,R } \\
\text { BFOX3,PNMT, } \\
\text { CNTNAP2,KC } \\
\text { NA1,NGFR,CA } \\
\text { CNA1A,CCK }\end{array}$ \\
\hline GO:0005788 & $\mathrm{CC}$ & $\begin{array}{l}\text { endoplasmic } \\
\text { reticulum lumen }\end{array}$ & $1.46 \mathrm{E}-03$ & $9.27 \mathrm{E}-02$ & $6.04 \mathrm{E}-01$ & $5.56 \mathrm{E}-01$ & 11 & $\begin{array}{l}\text { TRDN,F5,SPO } \\
\text { N1,SRL,COL4 } \\
\text { A6,COL8A2,C } \\
\text { OL13A1,WNT5 }\end{array}$ \\
\hline
\end{tabular}




\begin{tabular}{|c|c|c|c|c|c|c|c|c|}
\hline & & & & & & & & $\begin{array}{l}\text { A,ERP27,COL2 } \\
\text { 1A1,NTF4 }\end{array}$ \\
\hline GO:0034703 & $\mathrm{CC}$ & $\begin{array}{l}\text { cation channel } \\
\text { complex }\end{array}$ & $4.96 \mathrm{E}-03$ & $2.47 \mathrm{E}-01$ & $1.00 \mathrm{E}+00$ & $1.00 \mathrm{E}+00$ & 09 & $\begin{array}{l}\text { TRDN,SCN2B, } \\
\text { CNTNAP2,CA } \\
\text { CNA2D3,KCN } \\
\text { A1,KCNG1,KC } \\
\text { NJ16,KCNS2,C } \\
\text { ACNA1A }\end{array}$ \\
\hline GO:0005783 & $\mathrm{CC}$ & $\begin{array}{l}\text { endoplasmic } \\
\text { reticulum }\end{array}$ & $7.05 \mathrm{E}-03$ & $2.47 \mathrm{E}-01$ & $1.00 \mathrm{E}+00$ & $1.00 \mathrm{E}+00$ & 45 & $\begin{array}{l}\text { SPTLC3,LRAT, } \\
\text { CPED1,TRDN, } \\
\text { F5,PGR,S100A } \\
\text { 1,MGST1,SPO } \\
\text { N1,TMPRSS3,S } \\
\text { RL,PLA2G4A, } \\
\text { FGFR3,FLRT2, } \\
\text { HSD11B1,HSD } \\
\text { 17B2,SLC51A, } \\
\text { FXYD3,ALDO } \\
\text { B,COL4A6,CO } \\
\text { L8A2,COL13A } \\
\text { 1,FMO5,WNT5 } \\
\text { A,AGR2,XDH, } \\
\text { APOD,CSH1,C } \\
\text { SH2,AGMO,PR } \\
\text { SS50,CYP4B1, } \\
\text { KCNA1,ERP27 } \\
\text {,COL21A1,NG } \\
\text { FR,NTF4,CH25 } \\
\text { H,AGR3,OCA2 } \\
\text {,RNF175,CUB } \\
\text { N,TGFBR3,RA } \\
\text { SGRP1,SEZ6L }\end{array}$ \\
\hline GO:0090665 & $\mathrm{CC}$ & $\begin{array}{l}\text { glycoprotein } \\
\text { complex }\end{array}$ & 7.07E-03 & $2.47 \mathrm{E}-01$ & $1.00 \mathrm{E}+00$ & $1.00 \mathrm{E}+00$ & 03 & $\begin{array}{l}\text { SGCD,KRT19, } \\
\text { SNTG2 }\end{array}$ \\
\hline GO:0031226 & $\mathrm{CC}$ & $\begin{array}{l}\text { intrinsic } \\
\text { component of } \\
\text { plasma membrane }\end{array}$ & 0.02817 & $3.24 \mathrm{E}-01$ & $1.00 \mathrm{E}+00$ & $1.00 \mathrm{E}+00$ & 42 & $\begin{array}{l}\text { KLRF1,FCER1 } \\
\text { A,SCN2B,FGF } \\
\text { R3,FGFR2,FLR } \\
\text { T2,FXYD3,CO } \\
\text { L13A1,SGCD, } \\
\text { AQP4,CELSR1, } \\
\text { ART3,SLC15A } \\
\text { 1,CNTNAP2,S } \\
\text { LC13A5,ATP4 } \\
\text { A,IL6R,TRAB } \\
\text { D2B,PRSS8,G } \\
\text { ALR1,GAS1,SS } \\
\text { TR2,SLC16A12 } \\
\text {,SLC39A8,KC } \\
\text { NA1,KISS1R,K } \\
\text { CNG1,KCNJ12, } \\
\text { KCNJ16,NGFR } \\
\text {,KCNS2,GNRH } \\
\text { R,GPR12,FFAR } \\
\text { 3,ROR2,CCKB } \\
\text { R,TGFBR3,SL } \\
\text { C39A12,CD8B, } \\
\text { P2RY2,TSPAN }\end{array}$ \\
\hline
\end{tabular}




\begin{tabular}{|c|c|c|c|c|c|c|c|c|}
\hline & & & & & & & & 8,SLC6A14 \\
\hline GO:0005179 & MF & hormone activity & $4.87 \mathrm{E}-08$ & $3.94 \mathrm{E}-05$ & $2.87 \mathrm{E}-04$ & $3.94 \mathrm{E}-05$ & 14 & $\begin{array}{l}\text { CGB3,TSHB,P } \\
\text { OMC,CARTPT, } \\
\text { CSH1,CSH2,CS } \\
\text { HL1,FSHB,INH } \\
\text { BA,GPHA2,GH } \\
\text { 1,GH2,CCK,G } \\
\text { AL }\end{array}$ \\
\hline GO:0005102 & MF & $\begin{array}{l}\text { signaling receptor } \\
\text { binding }\end{array}$ & $4.24 \mathrm{E}-06$ & $1.71 \mathrm{E}-03$ & $1.25 \mathrm{E}-02$ & $3.43 \mathrm{E}-03$ & 54 & $\begin{array}{l}\text { ERBB4,CGB3, } \\
\text { S100A14,TSHB } \\
\text {,TAC4,TRDN,P } \\
\text { GR,S100B,EFE } \\
\text { MP1,PPP1R1B, } \\
\text { NR0B1,NGEF, } \\
\text { FGF1,FLRT2,S } \\
\text { FRP2,WNT5A, } \\
\text { AGR2,POMC, } \\
\text { ALKAL2,CAR } \\
\text { TPT,CSH1,CS } \\
\text { H2,CSHL1,ASI } \\
\text { P,FSHB,SLIT1, } \\
\text { RASL11B,IFN } \\
\text { E,IHH,IL6R,IN } \\
\text { HBA,LAMA1, } \\
\text { GPHA2,GH1,G } \\
\text { H2,BMP7,FOX } \\
\text { L2,NGFR,NTF } \\
\text { 4,ROR2,FAM3 } \\
\text { B,OGN,TF,CC } \\
\text { K,CCKBR,LEF } \\
\text { TY2,RSPO3,T } \\
\text { GFBR3,GAL,C } \\
\text { D8B,P2RY2,TS } \\
\text { PAN8,SNTG2, } \\
\text { LGI1 }\end{array}$ \\
\hline GO:0004854 & MF & $\begin{array}{l}\text { xanthine } \\
\text { dehydrogenase } \\
\text { activity }\end{array}$ & $3.25 \mathrm{E}-04$ & $6.58 \mathrm{E}-02$ & 4.79E-01 & $2.63 \mathrm{E}-01$ & 2 & $\mathrm{AOX} 1, \mathrm{XDH}$ \\
\hline GO:0016726 & MF & $\begin{array}{l}\text { oxidoreductase } \\
\text { activity, acting on } \\
\mathrm{CH} \text { or } \mathrm{CH} 2 \\
\text { groups, NAD or } \\
\text { NADP as acceptor }\end{array}$ & $3.25 \mathrm{E}-04$ & $6.58 \mathrm{E}-02$ & $4.79 \mathrm{E}-01$ & $2.63 \mathrm{E}-01$ & 2 & $\mathrm{AOX} 1, \mathrm{XDH}$ \\
\hline GO:0004864 & MF & $\begin{array}{l}\text { protein } \\
\text { phosphatase } \\
\text { inhibitor activity }\end{array}$ & $5.51 \mathrm{E}-04$ & 8.91E-02 & $6.48 \mathrm{E}-01$ & $4.46 \mathrm{E}-01$ & 5 & $\begin{array}{l}\text { PPP1R1B,PHA } \\
\text { CTR3,PHACT } \\
\text { R2,PPP1R17,PP } \\
\text { P1R27 }\end{array}$ \\
\hline GO:0019212 & MF & $\begin{array}{l}\text { phosphatase } \\
\text { inhibitor activity }\end{array}$ & $7.87 \mathrm{E}-04$ & $1.04 \mathrm{E}-01$ & $7.58 \mathrm{E}-01$ & $6.37 \mathrm{E}-01$ & 5 & $\begin{array}{l}\text { PPP1R1B,PHA } \\
\text { CTR3,PHACT } \\
\text { R2,PPP1R17,PP } \\
\text { P1R27 }\end{array}$ \\
\hline GO:0044548 & MF & $\begin{array}{l}\text { S100 protein } \\
\text { binding }\end{array}$ & $1.42 \mathrm{E}-03$ & $1.04 \mathrm{E}-01$ & $7.58 \mathrm{E}-01$ & $1.00 \mathrm{E}+00$ & 3 & $\begin{array}{l}\text { S100A1,S100B, } \\
\text { FGF1 }\end{array}$ \\
\hline
\end{tabular}




\begin{tabular}{|c|c|c|c|c|c|c|c|c|}
\hline GO:0008236 & MF & $\begin{array}{l}\text { serine-type } \\
\text { peptidase activity }\end{array}$ & $1.45 \mathrm{E}-03$ & $1.04 \mathrm{E}-01$ & $7.58 \mathrm{E}-01$ & $1.00 \mathrm{E}+00$ & 12 & $\begin{array}{l}\text { PCSK2,F5,FCN } \\
2, T M P R S S 3, P R \\
\text { SS45,PRSS50,C } \\
\text { TRB1,PRSS8,R } \\
\text { HBDL3,KLK11 } \\
\text {,CPXM2,KLK1 } \\
2\end{array}$ \\
\hline GO:0017171 & MF & $\begin{array}{l}\text { serine hydrolase } \\
\text { activity }\end{array}$ & $1.61 \mathrm{E}-03$ & $1.04 \mathrm{E}-01$ & $7.58 \mathrm{E}-01$ & $1.00 \mathrm{E}+00$ & 12 & $\begin{array}{l}\text { PCSK2,F5,FCN } \\
2, T M P R S S 3, P R \\
\text { SS45,PRSS50,C } \\
\text { TRB1,PRSS8,R } \\
\text { HBDL3,KLK11 } \\
\text {,CPXM2,KLK1 } \\
2\end{array}$ \\
\hline GO:0004252 & MF & $\begin{array}{l}\text { serine-type } \\
\text { endopeptidase } \\
\text { activity }\end{array}$ & $1.77 \mathrm{E}-03$ & $1.04 \mathrm{E}-01$ & $7.58 \mathrm{E}-01$ & $1.00 \mathrm{E}+00$ & 11 & $\begin{array}{l}\text { PCSK2,F5,FCN } \\
\text { 2,TMPRSS3,PR } \\
\text { SS45,PRSS50,C } \\
\text { TRB1,PRSS8,R } \\
\text { HBDL3,KLK11 } \\
\text {,KLK12 }\end{array}$ \\
\hline GO:0031781 & MF & $\begin{array}{l}\text { type } 3 \\
\text { melanocortin } \\
\text { receptor binding }\end{array}$ & $1.87 \mathrm{E}-03$ & $1.04 \mathrm{E}-01$ & 7.58E-01 & $1.00 \mathrm{E}+00$ & 2 & POMC,ASIP \\
\hline
\end{tabular}

Biological Process(BP), Cellular Component(CC) and Molecular Functions (MF)

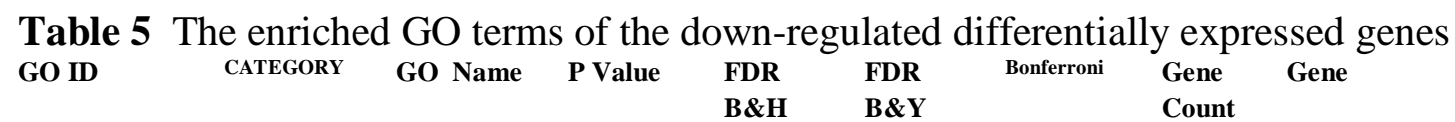

\begin{tabular}{|c|c|c|c|c|c|c|c|c|}
\hline GO:0000280 & BP & $\begin{array}{l}\text { nuclear } \\
\text { division }\end{array}$ & $2.87 \mathrm{E}-18$ & $6.44 \mathrm{E}-15$ & $5.58 \mathrm{E}-14$ & $9.45 \mathrm{E}-15$ & 41 & $\begin{array}{l}\text { NUSAP1,KIF18A,SPC25,RCC } \\
\text { 1,TTK,FANCA,FANCD2,CKS } \\
\text { 2,KLHL13,CLTC,PLK1,KIF23 } \\
\text {,BIRC5,SPC24,CENPH,TPX2, } \\
\text { RACGAP1,MTBP,CDCA5,PT } \\
\text { TG2,HAUS6,DLGAP5,PBK,R } \\
\text { AD21L1,NUF2,NEK2,PTTG3 } \\
\text { P,NCAPG,KIF15,BUB1,CCN } \\
\text { A1,KIF14,KIF11,RAD51,RAD } \\
\text { 51D,PRC1,EDN1,CCNF,CCN } \\
\text { G2,CCNB2,TOP2A }\end{array}$ \\
\hline
\end{tabular}

GO:0048285 BP $\quad$ organelle $\quad 3.91 \mathrm{E}-18 \quad 6.44 \mathrm{E}-15 \quad 5.58 \mathrm{E}-14 \quad 1.29 \mathrm{E}-14 \quad 42 \quad$ NUSAP1,KIF18A,SPC25,RCC fission $\quad 1$, TTK,FANCA,FANCD2,CKS 2,KLHL13,CLTC,PLK1,KIF23 ,BIRC5,SPC24,CENPH,TPX2, 


\begin{tabular}{|c|c|c|c|c|c|c|c|c|}
\hline GO:0007059 & $\mathrm{BP}$ & $\begin{array}{l}\text { chromosom } \\
\text { e } \\
\text { segregation }\end{array}$ & $1.04 \mathrm{E}-17$ & $1.14 \mathrm{E}-14$ & $9.91 \mathrm{E}-14$ & $3.43 \mathrm{E}-14$ & 31 & $\begin{array}{l}\text { NUSAP1,KIF18A,CENPA,HJ } \\
\text { URP,SPC25,RCC1,TTK,FAN } \\
\text { CD2,CENPM,PLK1,KIF23,BI } \\
\text { RC5,SPC24,CENPH,CENPI,R } \\
\text { ACGAP1,MTBP,CDCA5,PTT } \\
\text { G2,DLGAP5,RAD21L1,NUF2, } \\
\text { NEK2,PTTG3P,NCAPG,BUB1 } \\
\text {,KIF14,KIF11,CENPU,PRC1,T } \\
\text { OP2A }\end{array}$ \\
\hline GO:0098813 & $\mathrm{BP}$ & $\begin{array}{l}\text { nuclear } \\
\text { chromosom } \\
\text { e } \\
\text { segregation }\end{array}$ & $8.97 \mathrm{E}-17$ & 7.37E-14 & $6.40 \mathrm{E}-13$ & $2.95 \mathrm{E}-13$ & 28 & $\begin{array}{l}\text { NUSAP1,KIF18A,CENPA,SP } \\
\text { C25,TTK,FANCD2,CENPM,P } \\
\text { LK1,KIF23,BIRC5,SPC24,CE } \\
\text { NPH,CENPI,RACGAP1,MTB } \\
\text { P,CDCA5,PTTG2,DLGAP5,R } \\
\text { AD21L1,NUF2,NEK2,PTTG3 } \\
\text { P,NCAPG,BUB1,KIF14,CENP } \\
\text { U,PRC1,TOP2A }\end{array}$ \\
\hline GO:0022402 & $\mathrm{BP}$ & $\begin{array}{l}\text { cell cycle } \\
\text { process }\end{array}$ & $1.35 \mathrm{E}-15$ & $8.90 \mathrm{E}-13$ & $7.72 \mathrm{E}-12$ & $4.45 \mathrm{E}-12$ & 58 & $\begin{array}{l}\text { NUSAP1,CDKN3,KIF18A,CE } \\
\text { NPA,SPC25,RCC1,HMMR,TT } \\
\text { K,CDC45,FANCA,FANCD2,C } \\
\text { KS2,CENPM,KLHL13,ZC3H1 } \\
\text { 2D,CLTC,PLK1,FOXM1,KIF2 } \\
\text { 3,GTSE1,BIRC5,SPC24,CENP } \\
\text { H,CENPI,TPX2,RACGAP1,M } \\
\text { TBP,CDCA5,PTTG2,SOX4,H } \\
\text { AUS6,CYP27B1,DLGAP5,PB } \\
\text { K,RAD21L1,NUF2,MELK,NE } \\
\text { K2,PTTG3P,NCAPG,KIF15,B } \\
\text { UB1,CCNA1,KIF14,SLC39A5 } \\
\text {,KIF11,RAD51,RAD51D,CEN } \\
\text { PU,STMN1,PRC1,EDN1,KIF2 } \\
\text { 0A,CCNF,CCNG2,DDIAS,CC } \\
\text { NB2,TOP2A }\end{array}$ \\
\hline GO:0000819 & $\mathrm{BP}$ & $\begin{array}{l}\text { sister } \\
\text { chromatid } \\
\text { segregation }\end{array}$ & $2.90 \mathrm{E}-15$ & $1.59 \mathrm{E}-12$ & $1.38 \mathrm{E}-11$ & $9.53 \mathrm{E}-12$ & 24 & $\begin{array}{l}\text { NUSAP1,KIF18A,CENPA,SP } \\
\text { C25,TTK,CENPM,PLK1,KIF2 } \\
\text { 3,BIRC5,SPC24,CENPH,CEN } \\
\text { PI,RACGAP1,MTBP,CDCA5, } \\
\text { DLGAP5,NUF2,NEK2,NCAP } \\
\text { G,BUB1,KIF14,CENPU,PRC1, } \\
\text { TOP2A }\end{array}$ \\
\hline GO:1903047 & $\mathrm{BP}$ & $\begin{array}{l}\text { mitotic cell } \\
\text { cycle } \\
\text { process }\end{array}$ & $2.55 \mathrm{E}-14$ & $1.20 \mathrm{E}-11$ & $1.04 \mathrm{E}-10$ & $8.38 \mathrm{E}-11$ & 45 & $\begin{array}{l}\text { NUSAP1,CDKN3,KIF18A,CE } \\
\text { NPA,SPC25,RCC1,HMMR,TT } \\
\text { K,CDC45,CKS2,KLHL13,ZC3 } \\
\text { H12D,CLTC,PLK1,FOXM1,K } \\
\text { IF23,GTSE1,BIRC5,SPC24,CE } \\
\text { NPH,TPX2,RACGAP1,MTBP, } \\
\text { CDCA5,SOX4,HAUS6,DLGA } \\
\text { P5,PBK,NUF2,MELK,NEK2, } \\
\text { NCAPG,KIF15,BUB1,CCNA1 }\end{array}$ \\
\hline
\end{tabular}




GO:0007049 BP $\quad$ cell cycle $\quad 3.07 \mathrm{E}-14 \quad 1.26 \mathrm{E}-11 \quad 1.09 \mathrm{E}-10 \quad 1.01 \mathrm{E}-10 \quad 64$

GO:0000278 BP

GO:0051301 BP

GO:0000793 CC

GO:0000776 CC

GO:0000775 cell

division

condensed

chromosom

$5.65 \mathrm{E}-11$

$$
\text { e }
$$

mitotic cell cycle

$5.56 \mathrm{E}-13$

2.03E-1

$1.76 \mathrm{E}-0$

1.83E-09

45

kinetochore

$1.20 \mathrm{E}-10$

2.36E- 08

$1.55 \mathrm{E}-07$

4.72E-08

15

chromosom

4.29E-09

5.33E-07

$3.49 \mathrm{E}-06 \quad 1.69 \mathrm{E}-06$

16

centromeric

,KIF14,KIF11,STMN1,PRC1,E DN1,KIF20A,CCNF,CCNG2, CCNB2,TOP2A

NUSAP1,CDKN3,KIF18A,CE NPA,EXD1,HJURP,SPC25,RC C1,MND1,HMMR,TTK,CDC4 5,FANCA,FANCD2,CKS2,CE NPM,KLHL13,ZC3H12D,CLT C,PLK1,FOXM1,KIF23,GTSE 1,BIRC5,SPC24,CENPH,CEN PI,TPX2,RACGAP1,MTBP,PR R11,CDCA5,PTTG2,SOX4,H AUS6,CYP27B1,DLGAP5,PB K,RAD21L1,PARD6G,NUF2, MELK,PTP4A1,NEK2,PTTG3 P,NCAPG,KIF15,BUB1,CCN A1,KIF14,SLC39A5,KIF11,R AD51,RAD51D,CENPU,STM N1,PRC1,EDN1,KIF20A,CCN F,CCNG2,DDIAS,CCNB2,TO P2A

NUSAP1,CDKN3,KIF18A,CE NPA,SPC25,RCC1,HMMR,TT K,CDC45,CKS2,KLHL13,ZC3 H12D,CLTC,PLK1,FOXM1,K IF23,GTSE1,BIRC5,SPC24,CE NPH,TPX2,RACGAP1,MTBP, CDCA5,SOX4,HAUS6,DLGA P5,PBK,NUF2,MELK,NEK2, NCAPG,KIF15,BUB1,CCNA1 ,KIF14,KIF11,STMN1,PRC1,E DN1,KIF20A,CCNF,CCNG2, CCNB2,TOP2A

NUSAP1,SPC25,RCC1,TTK,C KS2,KLHL13,PLK1,KIF23,BI RC5,POU3F2,POU3F3,PPBP, SPC24,TPX2,RACGAP1,MTB $P, C D C A 5$, HAUS6,DLGAP5,P ARD6G,NUF2,NEK2,NCAPG, BUB1,CCNA1,KIF14,KIF11,S TMN1,PRC1,EDN1,KIF20A,C CNF,CCNG2,CCNB2,TOP2A

CENPA,HJURP,SPC25,RCC1, FANCD2,CENPM,PLK1,BIRC 5,SPC24,CENPH,CDCA5,RA D21L1,NUF2,NEK2,NCAPG, BUB1,RAD51,CENPU,TOP2A

KIF18A,CENPA,HJURP,SPC2 5,CENPM,PLK1,BIRC5,SPC2 4,CENPH,CENPI,MTBP,NUF 2,NEK2,BUB1,CENPU

KIF18A,CENPA,HJURP,SPC2 5,CENPM,PLK1,BIRC5,SPC2 4,CENPH,CENPI,MTBP,CDC A5,NUF2,NEK2,BUB1,CENP 


\begin{tabular}{|c|c|c|c|c|c|c|c|c|}
\hline & & region & & & & & & $\mathrm{U}$ \\
\hline GO:0000777 & $\mathrm{CC}$ & $\begin{array}{l}\text { condensed } \\
\text { chromosom } \\
\text { e } \\
\text { kinetochore }\end{array}$ & 5.42E-09 & 5.33E-07 & $3.49 \mathrm{E}-06$ & $2.13 \mathrm{E}-06$ & 12 & $\begin{array}{l}\text { CENPA,HJURP,SPC25,CENP } \\
\text { M,PLK1,BIRC5,SPC24,CENP } \\
\text { H,NUF2,NEK2,BUB1,CENPU }\end{array}$ \\
\hline GO:0005819 & $\mathrm{CC}$ & spindle & 7.20E-09 & $5.66 \mathrm{E}-07$ & $3.71 \mathrm{E}-06$ & $2.83 \mathrm{E}-06$ & 20 & $\begin{array}{l}\text { NUSAP1,KIF18A,TTK,CLTC, } \\
\text { PLK1,KIF23,POC1A,BIRC5,T } \\
\text { PX2,RACGAP1,HAUS6,DLG } \\
\text { AP5,PTP4A1,NEK2,KIF15,KI } \\
\text { F14,KIF11,RASSF10,PRC1,KI } \\
\text { F20A }\end{array}$ \\
\hline GO:0000779 & $\mathrm{CC}$ & $\begin{array}{l}\text { condensed } \\
\text { chromosom } \\
\text { e, } \\
\text { centromeric } \\
\text { region }\end{array}$ & $1.72 \mathrm{E}-08$ & $1.13 \mathrm{E}-06$ & 7.38E-06 & $6.76 \mathrm{E}-06$ & 12 & $\begin{array}{l}\text { CENPA,HJURP,SPC25,CENP } \\
\text { M,PLK1,BIRC5,SPC } 24, \text { CENP } \\
\text { H,NUF2,NEK2,BUB 1,CENPU }\end{array}$ \\
\hline GO:0005871 & $\mathrm{CC}$ & $\begin{array}{l}\text { kinesin } \\
\text { complex }\end{array}$ & $4.58 \mathrm{E}-08$ & $2.57 \mathrm{E}-06$ & $1.69 \mathrm{E}-05$ & $1.80 \mathrm{E}-05$ & 09 & $\begin{array}{l}\text { KIF18A,KIF23,KIF21B,KIF26 } \\
\text { A,DISC1,KIF15,KIF14,KIF11, } \\
\text { KIF20A }\end{array}$ \\
\hline GO:0015630 & $\mathrm{CC}$ & $\begin{array}{l}\text { microtubule } \\
\text { cytoskeleto } \\
\mathrm{n}\end{array}$ & $1.43 \mathrm{E}-07$ & 7.01E-06 & $4.59 \mathrm{E}-05$ & $5.61 \mathrm{E}-05$ & 38 & $\begin{array}{l}\text { NUSAP1,KIF18A,SPEF2,TTK } \\
\text {,CDC45,CLTC,C12orf66,PLK } \\
\text { 1,KIF23,POC1A,GTSE1,BIRC } \\
\text { 5,TPX2,RACGAP1,KIF21B,H } \\
\text { AUS6,KIF26A,DISC1,DLGAP } \\
\text { 5,DAB1,PTP4A1,NEK2,NCAP } \\
\text { G,KIF15,CCNA1,KIF14,RASS } \\
\text { F3,KIF11,RAD51,RAD51D,C } \\
\text { ENPU,RASSF10,STMN1,PRC } \\
\text { 1,KIF20A,CCNF,CCNB2,TOP } \\
\text { 2A }\end{array}$ \\
\hline GO:0005694 & $\mathrm{CC}$ & $\begin{array}{l}\text { chromosom } \\
\text { e }\end{array}$ & $4.51 \mathrm{E}-07$ & $1.97 \mathrm{E}-05$ & $1.29 \mathrm{E}-04$ & $1.77 \mathrm{E}-04$ & 33 & $\begin{array}{l}\text { NUSAP1,KIF18A,CENPA,HJ } \\
\text { URP,SPC25,RCC1,CDC45,FA } \\
\text { NCD2,SINHCAF,CENPM,CL } \\
\text { TC,PLK1,BIRC5,SPC24,CEN } \\
\text { PH,CENPI,MTBP,POLQ,CDC } \\
\text { A5,HMGB4,RAD21L1,NUF2, } \\
\text { TRIM24,NEK2,NCAPG,BUB1 } \\
\text {,PARPBP,RAD51,RAD51D,C } \\
\text { ENPU,RFC3,ASXL2,TOP2A }\end{array}$ \\
\hline GO:0044430 & $\mathrm{CC}$ & $\begin{array}{l}\text { cytoskeletal } \\
\text { part }\end{array}$ & $9.84 \mathrm{E}-07$ & $3.65 \mathrm{E}-05$ & $2.39 \mathrm{E}-04$ & $3.87 \mathrm{E}-04$ & 44 & $\begin{array}{l}\text { NUSAP1,KIF18A,SPEF2,TTK } \\
\text {,ADD2,CDC45,CLTC,PLK1,K } \\
\text { IF23,POC1A,CARMIL1,GTSE } \\
\text { 1,BIRC5,KRT87P,TPX2,RAC } \\
\text { GAP1,MYBPC1,KIF21B,HAU } \\
\text { S6,MYO1D,KIF26A,DISC1,D } \\
\text { LGAP5,DAB1,PTP4A1,NEK2, } \\
\text { NCAPG,KIF15,KIF14,RASSF } \\
\text { 3,KRTAP5- } \\
\text { 7,KIF11,RAD51,RAD51D,KR } \\
\text { T28,CENPU,RASSF10,STMN } \\
\text { 1,PRC1,KIF20A,CCNF,CCNB } \\
\text { 2,KRTAP1-5,TOP2A }\end{array}$ \\
\hline
\end{tabular}




\begin{tabular}{|c|c|c|c|c|c|c|c|c|}
\hline GO:0008017 & MF & $\begin{array}{l}\text { microtubule } \\
\text { binding }\end{array}$ & $6.06 \mathrm{E}-06$ & $2.40 \mathrm{E}-03$ & $1.73 \mathrm{E}-02$ & 4.63E-03 & 13 & $\begin{array}{l}\text { NUSAP1,KIF18A,PLK1,KIF2 } \\
\text { 3,BIRC5,RACGAP1,KIF21B, } \\
\text { KIF26A,KIF15,KIF14,KIF11,P } \\
\text { RC1,KIF20A }\end{array}$ \\
\hline GO:0003777 & MF & $\begin{array}{l}\text { microtubule } \\
\text { motor } \\
\text { activity }\end{array}$ & 8.30E-06 & $2.40 \mathrm{E}-03$ & $1.73 \mathrm{E}-02$ & $6.34 \mathrm{E}-03$ & 08 & $\begin{array}{l}\text { KIF18A,KIF23,KIF21B,KIF26 } \\
\text { A,KIF15,KIF14,KIF11,KIF20 } \\
\text { A }\end{array}$ \\
\hline GO:0015631 & MF & $\begin{array}{l}\text { tubulin } \\
\text { binding }\end{array}$ & $9.41 \mathrm{E}-06$ & $2.40 \mathrm{E}-03$ & $1.73 \mathrm{E}-02$ & 7.19E-03 & 15 & $\begin{array}{l}\text { NUSAP1,KIF18A,PLK1,KIF2 } \\
\text { 3,BIRC5,RACGAP1,KIF21B, } \\
\text { KIF26A,KIF15,KIF14,KIF11, } \\
\text { RAD51D,STMN1,PRC1,KIF2 } \\
\text { 0A }\end{array}$ \\
\hline GO:0008574 & MF & $\begin{array}{l}\text { ATP- } \\
\text { dependent } \\
\text { microtubule } \\
\text { motor } \\
\text { activity, } \\
\text { plus-end- } \\
\text { directed }\end{array}$ & $6.32 \mathrm{E}-05$ & $1.05 \mathrm{E}-02$ & 7.57E-02 & 4.83E-02 & 04 & KIF18A,KIF26A,KIF14,KIF11 \\
\hline GO:0003774 & MF & $\begin{array}{l}\text { motor } \\
\text { activity }\end{array}$ & $6.86 \mathrm{E}-05$ & $1.05 \mathrm{E}-02$ & $7.57 \mathrm{E}-02$ & $5.24 \mathrm{E}-02$ & 09 & $\begin{array}{l}\text { KIF18A,KIF23,KIF21B,MYO1 } \\
\text { D,KIF26A,KIF15,KIF14,KIF1 } \\
\text { 1,KIF20A }\end{array}$ \\
\hline GO:1990939 & MF & $\begin{array}{l}\text { ATP- } \\
\text { dependent } \\
\text { microtubule } \\
\text { motor } \\
\text { activity }\end{array}$ & $1.01 \mathrm{E}-04$ & $1.14 \mathrm{E}-02$ & 8.19E-02 & $7.71 \mathrm{E}-02$ & 04 & KIF18A,KIF26A,KIF14,KIF11 \\
\hline GO:0016887 & MF & $\begin{array}{l}\text { ATPase } \\
\text { activity }\end{array}$ & $1.04 \mathrm{E}-04$ & $1.14 \mathrm{E}-02$ & 8.19E-02 & $7.95 \mathrm{E}-02$ & 17 & $\begin{array}{l}\text { KIF18A,KIF23,POLQ,ATP2A } \\
\text { 3,ATP2B2,KIF21B,MYO1D,K } \\
\text { IF26A,DHX15,KIF15,KIF14,K } \\
\text { IF11,RAD51,RAD51D,RFC3, } \\
\text { KIF20A,TOP2A }\end{array}$ \\
\hline GO:0043142 & MF & $\begin{array}{l}\text { single- } \\
\text { stranded } \\
\text { DNA- } \\
\text { dependent } \\
\text { ATPase } \\
\text { activity }\end{array}$ & 7.57E-04 & $6.64 \mathrm{E}-02$ & 4.79E-01 & 5.79E-01 & 03 & POLQ,RAD51,RFC3 \\
\hline GO:0030165 & MF & $\begin{array}{l}\text { PDZ } \\
\text { domain } \\
\text { binding }\end{array}$ & $8.28 \mathrm{E}-04$ & $6.64 \mathrm{E}-02$ & 4.79E-01 & $6.33 \mathrm{E}-01$ & 07 & $\begin{array}{l}\text { ARHGAP29,NLGN1,CASK,A } \\
\text { TP2B2,KIF14,L1CAM,GRIK2 }\end{array}$ \\
\hline GO:0044877 & MF & $\begin{array}{l}\text { protein- } \\
\text { containing } \\
\text { complex } \\
\text { binding }\end{array}$ & 8.70E-04 & $6.64 \mathrm{E}-02$ & 4.79E-01 & $6.64 \mathrm{E}-01$ & 37 & $\begin{array}{l}\text { NUSAP1,DOK6,KIF18A,NRG } \\
\text { 1,CENPA,RBPJL,RCC1,ADD } \\
\text { 2,CDC45,CKS2,PLK1,KIF23, } \\
\text { VWF,CARMIL1,BIRC5,CAS } \\
\text { K,CENPH,RACGAP1,POLQ, } \\
\text { CDCA5,KIF21B,MYO1D,KIF } \\
\text { 26A,DISC1,TRIM24,YTHDF1 } \\
\text {,PRDM11,KIF15,KIF14,KIF11 } \\
\text {,RAD51,L1CAM,PRC1,KIF20 } \\
\text { A,TLE4,TOP2A,EPHB1 }\end{array}$ \\
\hline
\end{tabular}


Biological Process(BP), Cellular Component(CC) and Molecular Functions (MF)

Table 6 Topology table for up and down regulated genes

\begin{tabular}{|c|c|c|c|c|c|c|}
\hline Category & Node & Degree & Betweenness & Stress & Closeness & $\begin{array}{l}\text { Clustering } \\
\text { Coefficient }\end{array}$ \\
\hline Up & SALL1 & 20 & $6.73 \mathrm{E}-03$ & 599040 & 0.270852 & 0.026316 \\
\hline Up & BMP7 & 42 & $1.85 \mathrm{E}-02$ & 2547692 & 0.241805 & 0 \\
\hline Up & FAM83F & 2 & $5.08 \mathrm{E}-04$ & 43304 & 0.20099 & 0 \\
\hline Up & SGCD & 8 & $3.55 \mathrm{E}-03$ & 808402 & 0.171417 & 0 \\
\hline Up & C1orf168 & 1 & $0.00 \mathrm{E}+00$ & 0 & 1 & 0 \\
\hline Up & GRHL2 & 7 & $1.70 \mathrm{E}-03$ & 324668 & 0.243088 & 0 \\
\hline Up & TDO2 & 18 & $3.51 \mathrm{E}-03$ & 741118 & 0.221367 & 0 \\
\hline Up & SMR3B & 11 & $2.91 \mathrm{E}-03$ & 241848 & 0.209856 & 0 \\
\hline Up & SCRG1 & 1 & $0.00 \mathrm{E}+00$ & 0 & 0.171291 & 0 \\
\hline Up & IL20RA & 36 & $1.30 \mathrm{E}-02$ & 3240444 & 0.233049 & 0 \\
\hline Up & $\mathrm{FCN} 2$ & 7 & $2.56 \mathrm{E}-03$ & 197754 & 0.189402 & 0 \\
\hline Up & DPPA4 & 105 & $5.28 \mathrm{E}-02$ & 8404518 & 0.278355 & $1.90 \mathrm{E}-04$ \\
\hline Up & ATP4A & 91 & $4.54 \mathrm{E}-02$ & 4727962 & 0.263514 & 0 \\
\hline Up & GPR12 & 2 & $5.02 \mathrm{E}-04$ & 50910 & 0.215258 & 0 \\
\hline Up & SDK2 & 11 & $1.32 \mathrm{E}-03$ & 347148 & 0.206891 & 0 \\
\hline Up & SEPT14 & 9 & $3.56 \mathrm{E}-03$ & 546322 & 0.189457 & 0 \\
\hline Up & RSPO3 & 5 & $2.03 \mathrm{E}-03$ & 178044 & 0.172718 & 0 \\
\hline Up & SLC13A5 & 2 & $1.07 \mathrm{E}-04$ & 16672 & 0.203829 & 0 \\
\hline Up & FGFR2 & 93 & 5.79E-02 & 6781948 & 0.291174 & 0.003175 \\
\hline Up & MGST1 & 7 & $1.66 \mathrm{E}-03$ & 259370 & 0.209421 & 0 \\
\hline Up & FABP7 & 10 & $3.53 \mathrm{E}-03$ & 348144 & 0.198368 & 0 \\
\hline Up & PON3 & 2 & $1.00 \mathrm{E}+00$ & 2 & 1 & 0 \\
\hline Up & GAL & 9 & $2.89 \mathrm{E}-03$ & 341670 & 0.219065 & 0 \\
\hline Up & PAX6 & 49 & $2.00 \mathrm{E}-02$ & 1487900 & 0.266617 & 0.002775 \\
\hline Up & SYPL2 & 12 & $3.97 \mathrm{E}-03$ & 648624 & 0.216559 & 0 \\
\hline Up & LGI1 & 2 & $5.08 \mathrm{E}-04$ & 71602 & 0.185225 & 0 \\
\hline Up & SLC15A1 & 64 & $2.63 \mathrm{E}-02$ & 6462618 & 0.251196 & 0 \\
\hline Up & TMPRSS3 & 33 & $1.58 \mathrm{E}-02$ & 3314720 & 0.24797 & 0 \\
\hline Up & ROR2 & 49 & $1.54 \mathrm{E}-02$ & 2469916 & 0.257031 & 0 \\
\hline Up & DACT2 & 4 & $1.06 \mathrm{E}-03$ & 191246 & 0.177576 & 0 \\
\hline Up & SMTNL2 & 16 & $4.38 \mathrm{E}-03$ & 927394 & 0.241701 & 0 \\
\hline Up & KRT40 & 313 & $1.59 \mathrm{E}-01$ & 22565808 & 0.304829 & 0 \\
\hline Up & FBN2 & 24 & $6.88 \mathrm{E}-03$ & 1381044 & 0.220957 & 0 \\
\hline Up & NTF4 & 5 & $2.03 \mathrm{E}-03$ & 147964 & 0.13475 & 0 \\
\hline Up & GBA3 & 2 & $5.08 \mathrm{E}-04$ & 52696 & 0.148844 & 0 \\
\hline Up & SLIT1 & 2 & $5.08 \mathrm{E}-04$ & 49644 & 0.176676 & 0 \\
\hline Up & OR7A5 & 1 & $0.00 \mathrm{E}+00$ & 0 & 0.214321 & 0 \\
\hline
\end{tabular}




\begin{tabular}{|c|c|c|c|c|c|}
\hline Up & BBOX1 & 19 & $6.49 \mathrm{E}-03$ & 440000 & 0.226003 \\
\hline Up & ART3 & 24 & 8.13E-03 & 976682 & 0.239993 \\
\hline Up & ID4 & 13 & $3.35 \mathrm{E}-03$ & 782844 & 0.231447 \\
\hline Up & PLIN4 & 3 & $7.56 \mathrm{E}-05$ & 37378 & 0.213461 \\
\hline Up & TRDN & 37 & $1.31 \mathrm{E}-02$ & 2095642 & 0.247674 \\
\hline Up & CLIC5 & 8 & $2.39 \mathrm{E}-03$ & 360044 & 0.208391 \\
\hline Up & IRX1 & 1 & $0.00 \mathrm{E}+00$ & 0 & 0.200969 \\
\hline Up & FCER1A & 3 & $3.26 \mathrm{E}-05$ & 5636 & 0.185557 \\
\hline Up & NGEF & 21 & 7.48E-03 & 1282594 & 0.208005 \\
\hline Up & CCKBR & 17 & $6.42 \mathrm{E}-03$ & 1313620 & 0.225189 \\
\hline Up & ITLN1 & 11 & $3.22 \mathrm{E}-03$ & 584478 & 0.223274 \\
\hline Up & S100A1 & 23 & $5.90 \mathrm{E}-03$ & 824942 & 0.251533 \\
\hline Up & TMEM139 & 3 & $3.55 \mathrm{E}-03$ & 258886 & 0.184289 \\
\hline Up & PMAIP1 & 14 & 4.74E-03 & 615246 & 0.230621 \\
\hline Up & GHRHR & 1 & $0.00 \mathrm{E}+00$ & 0 & 1 \\
\hline Up & $\mathrm{XDH}$ & 2 & $5.08 \mathrm{E}-04$ & 44858 & 0.200601 \\
\hline Up & TRIM29 & 104 & $6.13 \mathrm{E}-02$ & 9041322 & 0.297553 \\
\hline Up & UGT2A2 & 1 & $0.00 \mathrm{E}+00$ & 0 & 1 \\
\hline Up & SOX5 & 37 & $1.43 \mathrm{E}-02$ & 1508376 & 0.275378 \\
\hline Up & S100A1 & 23 & $5.90 \mathrm{E}-03$ & 824942 & 0.251533 \\
\hline Up & GALR1 & 1 & $0.00 \mathrm{E}+00$ & 0 & 0.179707 \\
\hline Up & GAS1 & 3 & $1.01 \mathrm{E}-03$ & 43314 & 0.226575 \\
\hline Up & PPP1R17 & 1 & $0.00 \mathrm{E}+00$ & 0 & 0.214321 \\
\hline Up & IFNE & 10 & $2.80 \mathrm{E}-03$ & 416368 & 0.217901 \\
\hline Up & CELSR1 & 17 & $4.71 \mathrm{E}-03$ & 588542 & 0.223388 \\
\hline Up & C4orf19 & 7 & 5.94E-04 & 75814 & 0.20404 \\
\hline Up & POMC & 13 & $5.46 \mathrm{E}-03$ & 738136 & 0.221941 \\
\hline Up & LRAT & 3 & $0.00 \mathrm{E}+00$ & 0 & 1 \\
\hline Up & KLK11 & 27 & $1.36 \mathrm{E}-02$ & 2408872 & 0.236335 \\
\hline Up & F5 & 15 & 4.61E-03 & 881738 & 0.220265 \\
\hline Up & CNTNAP2 & 8 & $2.06 \mathrm{E}-03$ & 345082 & 0.217348 \\
\hline Up & ENPP6 & 40 & 1.39E-02 & 3610524 & 0.233408 \\
\hline Up & GBP6 & 3 & $1.02 \mathrm{E}-03$ & 53086 & 0.167077 \\
\hline Up & OBP2A & 7 & $3.04 \mathrm{E}-03$ & 662994 & 0.170129 \\
\hline Up & RBP4 & 15 & $4.72 \mathrm{E}-03$ & 550068 & 0.226992 \\
\hline Up & PPP1R27 & 1 & $0.00 \mathrm{E}+00$ & 0 & 0.166294 \\
\hline Up & ITIH2 & 16 & $5.09 \mathrm{E}-03$ & 851246 & 0.219884 \\
\hline Up & NEURL3 & 1 & $0.00 \mathrm{E}+00$ & 0 & 1 \\
\hline $\mathrm{Up}$ & CLIC5 & 8 & $2.39 \mathrm{E}-03$ & 360044 & 0.208391 \\
\hline Up & PPP1R27 & 1 & $0.00 \mathrm{E}+00$ & 0 & 0.166294 \\
\hline Up & RASL11B & 6 & $1.08 \mathrm{E}-03$ & 251748 & 0.233312 \\
\hline Up & $\mathrm{KCNJ} 12$ & 17 & 4.14E-03 & 1228964 & 0.207786 \\
\hline Up & AQP4 & 3 & $5.64 \mathrm{E}-04$ & 54624 & 0.198508 \\
\hline
\end{tabular}




\begin{tabular}{|c|c|c|c|c|c|}
\hline Up & CD8B & 2 & $5.08 \mathrm{E}-04$ & 43304 & 0.20099 \\
\hline Up & P2RY2 & 4 & $9.10 \mathrm{E}-04$ & 137648 & 0.233077 \\
\hline Up & IL22RA1 & 7 & $2.15 \mathrm{E}-03$ & 456438 & 0.199181 \\
\hline Up & RNF207 & 4 & $6.33 \mathrm{E}-04$ & 99124 & 0.213496 \\
\hline Up & ADAMTS18 & 5 & $7.80 \mathrm{E}-04$ & 175548 & 0.216858 \\
\hline Up & NGFR & 46 & $1.93 \mathrm{E}-02$ & 2626710 & 0.258024 \\
\hline Up & KCNJ16 & 1 & $0.00 \mathrm{E}+00$ & 0 & 1 \\
\hline Up & EFEMP1 & 56 & $3.53 \mathrm{E}-02$ & 3383126 & 0.271749 \\
\hline Up & $\mathrm{CHN} 2$ & 13 & $3.42 \mathrm{E}-03$ & 578204 & 0.234506 \\
\hline Up & GPHA2 & 21 & $6.38 \mathrm{E}-03$ & 1083752 & 0.214718 \\
\hline Up & GHRHR & 1 & $0.00 \mathrm{E}+00$ & 0 & 1 \\
\hline Up & KRT40 & 313 & $1.59 \mathrm{E}-01$ & 22565808 & 0.304829 \\
\hline Up & C6orf132 & 1 & $0.00 \mathrm{E}+00$ & 0 & 0.195135 \\
\hline Up & NRAP & 7 & $2.54 \mathrm{E}-03$ & 723962 & 0.182437 \\
\hline Up & PHACTR2 & 16 & $5.85 \mathrm{E}-03$ & 1042524 & 0.242609 \\
\hline Up & EVC2 & 86 & 4.01E-02 & 3002590 & 0.269961 \\
\hline Up & FOXL2 & 70 & $2.86 \mathrm{E}-02$ & 8356260 & 0.257889 \\
\hline Up & FAM3B & 9 & $5.47 \mathrm{E}-03$ & 400338 & 0.24077 \\
\hline Up & DLK1 & 69 & $3.09 \mathrm{E}-02$ & 5339202 & 0.257485 \\
\hline Up & BLID & 12 & $2.22 \mathrm{E}-03$ & 427182 & 0.219345 \\
\hline Up & CALB1 & 8 & $1.87 \mathrm{E}-03$ & 281870 & 0.227635 \\
\hline Up & OGN & 10 & $3.36 \mathrm{E}-03$ & 588086 & 0.221218 \\
\hline Up & $\mathrm{TF}$ & 59 & $3.30 \mathrm{E}-02$ & 5546776 & 0.282609 \\
\hline Up & SMAD9 & 114 & 7.87E-02 & 8103704 & 0.303093 \\
\hline $\mathrm{Up}$ & SOX2 & 355 & 2.07E-01 & 37524600 & 0.318174 \\
\hline Up & KRT14 & 53 & $2.76 \mathrm{E}-02$ & 6537242 & 0.277277 \\
\hline Up & DLGAP2 & 14 & 8.62E-03 & 607018 & 0.259128 \\
\hline Up & LRRC2 & 2 & $1.61 \mathrm{E}-05$ & 2042 & 0.164853 \\
\hline Up & AMOT & 111 & $6.81 \mathrm{E}-02$ & 13889668 & 0.286702 \\
\hline Up & ZNF385C & 7 & $2.12 \mathrm{E}-03$ & 396460 & 0.198308 \\
\hline Up & $\mathrm{CCK}$ & 2 & $5.08 \mathrm{E}-04$ & 109288 & 0.183825 \\
\hline Up & HSD11B1 & 16 & $5.27 \mathrm{E}-03$ & 800492 & 0.218251 \\
\hline Up & SGCD & 8 & $3.55 \mathrm{E}-03$ & 808402 & 0.171417 \\
\hline Up & PRSS8 & 1 & $0.00 \mathrm{E}+00$ & 0 & 1 \\
\hline Up & C6orf118 & 5 & $1.11 \mathrm{E}-03$ & 182260 & 0.220228 \\
\hline Up & LAD1 & 7 & $2.00 \mathrm{E}-03$ & 282468 & 0.222492 \\
\hline Up & MYL10 & 3 & $3.70 \mathrm{E}-04$ & 73004 & 0.215777 \\
\hline Up & CRISP2 & 5 & $2.02 \mathrm{E}-03$ & 232114 & 0.218082 \\
\hline $\mathrm{Up}$ & IHH & 2 & $5.08 \mathrm{E}-04$ & 70496 & 0.159532 \\
\hline Up & MCOLN2 & 1 & $0.00 \mathrm{E}+00$ & 0 & 1 \\
\hline Up & SLC39A12 & 70 & $3.22 \mathrm{E}-02$ & 4986186 & 0.249178 \\
\hline Up & VIT & 2 & $5.08 \mathrm{E}-04$ & 69608 & 0.209376 \\
\hline Up & PPP1R17 & 1 & $0.00 \mathrm{E}+00$ & 0 & 0.214321 \\
\hline
\end{tabular}




\begin{tabular}{|c|c|c|c|c|c|c|}
\hline Up & TSHB & 15 & $6.40 \mathrm{E}-03$ & 1264174 & 0.237848 & 0 \\
\hline Up & GADD45G & 56 & $2.74 \mathrm{E}-02$ & 5419566 & 0.271843 & 0 \\
\hline Up & FXYD3 & 7 & $1.80 \mathrm{E}-03$ & 219094 & 0.20662 & 0 \\
\hline Up & LIX1 & 2 & $1.00 \mathrm{E}+00$ & 2 & 1 & 0 \\
\hline Up & PRSS45 & 3 & $1.00 \mathrm{E}+00$ & 6 & 1 & 0 \\
\hline Up & MFAP4 & 6 & $1.38 \mathrm{E}-03$ & 246964 & 0.216631 & 0 \\
\hline Up & SPON1 & 3 & $1.02 \mathrm{E}-03$ & 109098 & 0.214367 & 0 \\
\hline Up & NFIX & 22 & $5.02 \mathrm{E}-03$ & 1428620 & 0.232403 & 0 \\
\hline $\mathrm{Up}$ & COL8A2 & 18 & $6.18 \mathrm{E}-03$ & 653142 & 0.221205 & 0 \\
\hline Up & GNRHR & 1 & $0.00 \mathrm{E}+00$ & 0 & 1 & 0 \\
\hline Up & SULT1C2 & 10 & 4.91E-03 & 344354 & 0.215375 & 0 \\
\hline Up & CA8 & 33 & $1.30 \mathrm{E}-02$ & 1324240 & 0.262215 & 0 \\
\hline Up & CHRDL1 & 6 & 7.97E-04 & 108788 & 0.198089 & 0 \\
\hline Up & MEGF6 & 6 & $1.43 \mathrm{E}-03$ & 100130 & 0.228083 & 0 \\
\hline Up & RPRM & 9 & $3.81 \mathrm{E}-03$ & 39669 & 0.211331 & 0 \\
\hline Up & OCA2 & 3 & $8.78 \mathrm{E}-05$ & 7862 & 0.168788 & 0 \\
\hline Up & MPPED1 & 26 & 8.03E-03 & 1622472 & 0.222492 & 0 \\
\hline Up & CARTPT & 3 & $1.00 \mathrm{E}+00$ & 6 & 1 & 0 \\
\hline Up & CSH1 & 13 & $5.61 \mathrm{E}-03$ & 475316 & 0.257014 & 0 \\
\hline Up & GALNT9 & 1 & $0.00 \mathrm{E}+00$ & 0 & 0.189969 & 0 \\
\hline Up & OGN & 10 & $3.36 \mathrm{E}-03$ & 588086 & 0.221218 & 0 \\
\hline Up & FAM153B & 14 & $5.40 \mathrm{E}-03$ & 935250 & 0.2049 & 0 \\
\hline Up & GADD45G & 56 & $2.74 \mathrm{E}-02$ & 5419566 & 0.271843 & 0 \\
\hline Up & VSNL1 & 10 & $1.31 \mathrm{E}-03$ & 162184 & 0.199151 & 0 \\
\hline Up & KISS1R & 3 & $1.02 \mathrm{E}-03$ & 144830 & 0.182319 & 0 \\
\hline Up & FGFR3 & 49 & $2.12 \mathrm{E}-02$ & 2766462 & 0.268434 & 0 \\
\hline Up & WNT5A & 22 & $7.80 \mathrm{E}-03$ & 881282 & 0.206349 & 0 \\
\hline Up & CD8B & 2 & $5.08 \mathrm{E}-04$ & 43304 & 0.20099 & 0 \\
\hline Up & SFRP2 & 17 & $3.65 \mathrm{E}-03$ & 988952 & 0.223769 & 0 \\
\hline Up & HSPB3 & 24 & $9.14 \mathrm{E}-03$ & 1066592 & 0.24811 & 0 \\
\hline Up & PAX6 & 49 & $2.00 \mathrm{E}-02$ & 1487900 & 0.266617 & 0.002775 \\
\hline Up & CYBRD1 & 21 & 7.61E-03 & 1486198 & 0.221255 & 0 \\
\hline Up & FXYD3 & 7 & $1.80 \mathrm{E}-03$ & 219094 & 0.20662 & 0 \\
\hline Up & PGR & 68 & $3.40 \mathrm{E}-02$ & 5124700 & 0.28689 & 0 \\
\hline Up & HSD17B2 & 2 & $5.08 \mathrm{E}-04$ & 61020 & 0.152911 & 0 \\
\hline Up & OPCML & 11 & $9.23 \mathrm{E}-03$ & 415810 & 0.253949 & 0 \\
\hline Up & RFX4 & 4 & $5.08 \mathrm{E}-04$ & 68768 & 0.194519 & 0 \\
\hline Up & AGR3 & 14 & $3.65 \mathrm{E}-03$ & 622642 & 0.231135 & 0 \\
\hline Up & SLIT1 & 2 & $5.08 \mathrm{E}-04$ & 49644 & 0.176676 & 0 \\
\hline Up & SOX9 & 19 & 4.66E-03 & 1135172 & 0.228918 & 0 \\
\hline Up & CSHL1 & 3 & $1.02 \mathrm{E}-03$ & 142558 & 0.195484 & 0 \\
\hline Up & CHST9 & 4 & $4.30 \mathrm{E}-04$ & 57912 & 0.2136 & 0 \\
\hline Up & SULT1C2 & 10 & 4.91E-03 & 344354 & 0.215375 & 0 \\
\hline
\end{tabular}




\begin{tabular}{|c|c|c|c|c|c|}
\hline Up & DYDC2 & 1 & $0.00 \mathrm{E}+00$ & 0 & 1 \\
\hline Up & SLIT1 & 4 & $1.52 \mathrm{E}-03$ & 98748 & 0.177265 \\
\hline Up & SNTG2 & 14 & 4.17E-03 & 686616 & 0.201051 \\
\hline Up & ZNF750 & 1 & $0.00 \mathrm{E}+00$ & 0 & 0.196381 \\
\hline Up & TAC4 & 3 & $1.00 \mathrm{E}+00$ & 6 & 1 \\
\hline Up & PTER & 14 & $4.59 \mathrm{E}-03$ & 989784 & 0.223477 \\
\hline Up & CTRB1 & 2 & $1.00 \mathrm{E}+00$ & 2 & 1 \\
\hline Up & COL21A1 & 2 & $0.00 \mathrm{E}+00$ & 0 & 0 \\
\hline Up & ATF7IP2 & 4 & 7.03E-04 & 79612 & 0.215258 \\
\hline Up & NEFM & 50 & $2.12 \mathrm{E}-02$ & 4143078 & 0.272012 \\
\hline Up & S100A14 & 21 & 8.84E-03 & 986808 & 0.265288 \\
\hline Up & AOX1 & 6 & $1.58 \mathrm{E}-03$ & 130420 & 0.202895 \\
\hline Up & KLRF1 & 5 & $6.70 \mathrm{E}-04$ & 77804 & 0.192503 \\
\hline Up & CCDC68 & 13 & $5.14 \mathrm{E}-03$ & 436104 & 0.208269 \\
\hline Up & CNKSR3 & 9 & $2.74 \mathrm{E}-03$ & 263976 & 0.196244 \\
\hline Up & CRYM & 4 & $5.65 \mathrm{E}-04$ & 44096 & 0.209666 \\
\hline Up & MYZAP & 1 & $0.00 \mathrm{E}+00$ & 0 & 0.213484 \\
\hline Up & PDE6A & 4 & $1.43 \mathrm{E}-04$ & 8434 & 0.238597 \\
\hline Up & INHBA & 8 & $2.57 \mathrm{E}-03$ & 734598 & 0.176407 \\
\hline Up & CNTNAP3 & 33 & $1.34 \mathrm{E}-02$ & 1433542 & 0.235939 \\
\hline Up & FAM153C & 2 & $5.08 \mathrm{E}-04$ & 85808 & 0.170078 \\
\hline Up & C6orf141 & 74 & $3.72 \mathrm{E}-02$ & 9207512 & 0.260292 \\
\hline Up & RUNX1 & 79 & $3.50 \mathrm{E}-02$ & 4622022 & 0.286015 \\
\hline Up & TRIM71 & 17 & $6.52 \mathrm{E}-03$ & 713150 & 0.220623 \\
\hline Up & PNMT & 3 & $5.88 \mathrm{E}-04$ & 55878 & 0.215493 \\
\hline Up & PRSS50 & 25 & $9.88 \mathrm{E}-03$ & 1981826 & 0.256663 \\
\hline Up & SLC47A1 & 27 & $1.28 \mathrm{E}-02$ & 765214 & 0.222832 \\
\hline Up & PHACTR3 & 29 & $1.17 \mathrm{E}-02$ & 1887264 & 0.234506 \\
\hline Up & IL20RA & 36 & $1.30 \mathrm{E}-02$ & 3240444 & 0.233049 \\
\hline Up & LEFTY2 & 3 & $1.02 \mathrm{E}-03$ & 95398 & 0.147528 \\
\hline Up & RHBDL3 & 4 & $1.52 \mathrm{E}-03$ & 323400 & 0.165874 \\
\hline Up & GH1 & 85 & 3.79E-02 & 5815610 & 0.26716 \\
\hline Up & STRA6 & 2 & $5.08 \mathrm{E}-04$ & 57684 & 0.184652 \\
\hline Up & FRAS1 & 33 & $1.76 \mathrm{E}-02$ & 1896950 & 0.244978 \\
\hline Up & CT45A1 & 3 & $1.00 \mathrm{E}+00$ & 6 & 1 \\
\hline Up & FGFR3 & 49 & 2.12E-02 & 2766462 & 0.268434 \\
\hline Up & EYA4 & 26 & $6.49 \mathrm{E}-03$ & 1286994 & 0.239075 \\
\hline Up & AMZ1 & 12 & $3.35 \mathrm{E}-03$ & 644006 & 0.193934 \\
\hline $\mathrm{Up}$ & CACNA1A & 98 & $5.07 \mathrm{E}-02$ & 4392082 & 0.272841 \\
\hline Up & CA12 & 13 & 3.67E-03 & 625692 & 0.218106 \\
\hline Up & GAS7 & 28 & $9.64 \mathrm{E}-03$ & 1499288 & 0.218894 \\
\hline Up & HPGD & 1 & $0.00 \mathrm{E}+00$ & 0 & 0.214321 \\
\hline Up & FMO5 & 5 & $9.24 \mathrm{E}-04$ & 141874 & 0.217516 \\
\hline
\end{tabular}




\begin{tabular}{|c|c|c|c|c|c|}
\hline Up & SCGB1D2 & 9 & $2.67 \mathrm{E}-03$ & 252442 & 0.209376 \\
\hline Up & PLIN5 & 6 & $2.54 \mathrm{E}-03$ & 488350 & 0.178558 \\
\hline Up & KCNA1 & 7 & $2.68 \mathrm{E}-03$ & 237802 & 0.195435 \\
\hline Up & ALDOB & 28 & $1.11 \mathrm{E}-02$ & 1644614 & 0.253084 \\
\hline Up & RHOD & 19 & $7.80 \mathrm{E}-03$ & 1084918 & 0.189786 \\
\hline Up & GJB7 & 51 & $2.16 \mathrm{E}-02$ & 2788870 & 0.243992 \\
\hline Up & ALDH1A3 & 9 & $2.42 \mathrm{E}-03$ & 378026 & 0.220327 \\
\hline Up & EYA2 & 88 & $4.15 \mathrm{E}-02$ & 6323538 & 0.281237 \\
\hline Up & KRT19 & 59 & $2.60 \mathrm{E}-02$ & 7305954 & 0.276926 \\
\hline Up & LY6H & 3 & $5.57 \mathrm{E}-04$ & 60178 & 0.176092 \\
\hline Up & MYBPHL & 7 & $2.32 \mathrm{E}-03$ & 246776 & 0.198308 \\
\hline Up & RASGRP1 & 4 & $3.51 \mathrm{E}-04$ & 53352 & 0.195019 \\
\hline Up & GH2 & 2 & $8.82 \mathrm{E}-06$ & 644 & 0.197325 \\
\hline Up & FERMT1 & 15 & $4.58 \mathrm{E}-03$ & 653358 & 0.227097 \\
\hline Up & ASXL3 & 2 & $5.08 \mathrm{E}-04$ & 44514 & 0.172627 \\
\hline Up & TEPP & 1 & $0.00 \mathrm{E}+00$ & 0 & 0.209354 \\
\hline Up & PDZRN3 & 11 & 4.13E-03 & 858088 & 0.195387 \\
\hline Up & KCNS2 & 1 & $0.00 \mathrm{E}+00$ & 0 & 0.148569 \\
\hline Up & VANGL2 & 33 & $1.46 \mathrm{E}-02$ & 1934604 & 0.232321 \\
\hline Up & PRDM16 & 6 & $1.44 \mathrm{E}-03$ & 306010 & 0.223972 \\
\hline Up & PLAC1 & 20 & $6.67 \mathrm{E}-03$ & 1205836 & 0.224957 \\
\hline Up & SH3BP4 & 43 & $1.67 \mathrm{E}-02$ & 2668100 & 0.262618 \\
\hline Up & COL4A6 & 9 & 4.29E-03 & 288964 & 0.222241 \\
\hline Up & NEUROD1 & 15 & $3.84 \mathrm{E}-03$ & 758734 & 0.219016 \\
\hline Up & IL6R & 17 & $6.73 \mathrm{E}-03$ & 876290 & 0.223845 \\
\hline Up & FSHB & 2 & $1.00 \mathrm{E}+00$ & 2 & 1 \\
\hline Up & $\mathrm{CSH} 2$ & 13 & $5.61 \mathrm{E}-03$ & 475316 & 0.257014 \\
\hline Up & CNTNAP3B & 16 & $7.15 \mathrm{E}-03$ & 582922 & 0.190741 \\
\hline Up & CHRDL1 & 6 & 7.97E-04 & 108788 & 0.198089 \\
\hline Up & NWD1 & 1 & $0.00 \mathrm{E}+00$ & 0 & 0.175339 \\
\hline Up & WWC2 & 18 & $3.48 \mathrm{E}-03$ & 975000 & 0.230041 \\
\hline Up & APOD & 50 & $3.56 \mathrm{E}-02$ & 2147634 & 0.271412 \\
\hline Up & GJB7 & 51 & $2.16 \mathrm{E}-02$ & 2788870 & 0.243992 \\
\hline Up & RXRG & 32 & $1.14 \mathrm{E}-02$ & 2426724 & 0.228268 \\
\hline Up & RNF175 & 2 & $2.24 \mathrm{E}-05$ & 1134 & 0.179355 \\
\hline Up & IGSF11 & 2 & $0.00 \mathrm{E}+00$ & 0 & 0 \\
\hline Up & $\mathrm{SCN} 2 \mathrm{~B}$ & 86 & $3.56 \mathrm{E}-02$ & 7895890 & 0.255896 \\
\hline Up & OVOL2 & 1 & $0.00 \mathrm{E}+00$ & 0 & 0.216108 \\
\hline Up & CCDC158 & 12 & $2.91 \mathrm{E}-03$ & 441576 & 0.225705 \\
\hline Up & ASXL3 & 2 & $5.08 \mathrm{E}-04$ & 44514 & 0.172627 \\
\hline Up & CYP4B1 & 2 & $1.00 \mathrm{E}+00$ & 2 & 1 \\
\hline Up & DLGAP2 & 14 & $8.62 \mathrm{E}-03$ & 607018 & 0.259128 \\
\hline Up & TPH1 & 7 & $1.87 \mathrm{E}-03$ & 396120 & 0.227635 \\
\hline
\end{tabular}




\begin{tabular}{|c|c|c|c|c|c|}
\hline Up & AOX1 & 6 & $1.58 \mathrm{E}-03$ & 130420 & 0.202895 \\
\hline Up & SLC39A8 & 26 & $1.05 \mathrm{E}-02$ & 1259100 & 0.220179 \\
\hline Up & S100B & 33 & $1.07 \mathrm{E}-02$ & 1384834 & 0.252922 \\
\hline Up & SLPI & 7 & $4.10 \mathrm{E}-03$ & 388202 & 0.209143 \\
\hline Up & PGR & 68 & $3.40 \mathrm{E}-02$ & 5124700 & 0.28689 \\
\hline Up & SSTR2 & 7 & $2.14 \mathrm{E}-03$ & 321652 & 0.229425 \\
\hline Up & FLRT2 & 3 & $0.00 \mathrm{E}+00$ & 0 & 1 \\
\hline Up & C2orf71 & 5 & $2.03 \mathrm{E}-03$ & 289652 & 0.182386 \\
\hline Up & COL13A1 & 2 & $0.00 \mathrm{E}+00$ & 0 & 0 \\
\hline Up & CACNA2D3 & 1 & $0.00 \mathrm{E}+00$ & 0 & 1 \\
\hline Up & NR0B1 & 15 & $5.59 \mathrm{E}-03$ & 309134 & 0.247425 \\
\hline Up & CALB2 & 4 & $1.64 \mathrm{E}-05$ & 1926 & 0.187706 \\
\hline Up & STMN2 & 27 & $1.22 \mathrm{E}-02$ & 1546160 & 0.247254 \\
\hline Up & SCGB1D1 & 71 & $4.35 \mathrm{E}-02$ & 6754916 & 0.254063 \\
\hline Up & FGF1 & 27 & $1.23 \mathrm{E}-02$ & 832698 & 0.262916 \\
\hline Up & KCNA1 & 7 & $2.68 \mathrm{E}-03$ & 237802 & 0.195435 \\
\hline Up & FOXL2 & 70 & $2.86 \mathrm{E}-02$ & 8356260 & 0.257889 \\
\hline Up & MAP3K15 & 7 & $1.18 \mathrm{E}-03$ & 196726 & 0.213172 \\
\hline Up & MKX & 10 & $2.04 \mathrm{E}-03$ & 492958 & 0.220302 \\
\hline Up & TXLNB & 43 & $1.53 \mathrm{E}-02$ & 4523946 & 0.233769 \\
\hline Up & $\mathrm{T}$ & 1 & $0.00 \mathrm{E}+00$ & 0 & 1 \\
\hline Up & ERBB4 & 84 & $3.50 \mathrm{E}-02$ & 8327424 & 0.269131 \\
\hline Up & PPP1R1B & 8 & $1.69 \mathrm{E}-03$ & 176268 & 0.187885 \\
\hline Up & PCSK2 & 2 & $0.00 \mathrm{E}+00$ & 0 & 0 \\
\hline Up & DMKN & 7 & $2.21 \mathrm{E}-03$ & 390378 & 0.236123 \\
\hline Up & TGFBR3 & 22 & $9.08 \mathrm{E}-03$ & 805594 & 0.23413 \\
\hline Up & MN1 & 3 & 4.47E-05 & 9412 & 0.20941 \\
\hline Up & SLIT1 & 2 & $5.08 \mathrm{E}-04$ & 49644 & 0.176676 \\
\hline Up & KCNG1 & 5 & $1.09 \mathrm{E}-03$ & 200078 & 0.194567 \\
\hline Up & MEGF11 & 2 & $5.16 \mathrm{E}-05$ & 7530 & 0.192871 \\
\hline Up & PLA2G4A & 35 & $1.68 \mathrm{E}-02$ & 3125428 & 0.264079 \\
\hline Up & ERP27 & 5 & $6.80 \mathrm{E}-04$ & 96440 & 0.20941 \\
\hline Up & GPR37L1 & 1 & $0.00 \mathrm{E}+00$ & 0 & 1 \\
\hline Up & SMR3B & 11 & 2.91E-03 & 241848 & 0.209856 \\
\hline Up & CLIC3 & 9 & $3.25 \mathrm{E}-03$ & 417244 & 0.21813 \\
\hline Up & SYT12 & 16 & $5.27 \mathrm{E}-03$ & 975528 & 0.226783 \\
\hline Up & CUBN & 10 & $2.40 \mathrm{E}-03$ & 351968 & 0.206793 \\
\hline Up & PNMA3 & 5 & 7.32E-04 & 128516 & 0.189685 \\
\hline Up & FAM198A & 3 & $9.84 \mathrm{E}-05$ & 8632 & 0.182912 \\
\hline Up & FAM124A & 29 & $1.00 \mathrm{E}-02$ & 2057940 & 0.226796 \\
\hline Up & CPLX3 & 17 & $7.35 \mathrm{E}-03$ & 422424 & 0.250732 \\
\hline Up & ADARB2 & 9 & $2.11 \mathrm{E}-03$ & 368998 & 0.226171 \\
\hline Up & DMKN & 7 & $2.21 \mathrm{E}-03$ & 390378 & 0.236123 \\
\hline
\end{tabular}




\begin{tabular}{|c|c|c|c|c|c|c|}
\hline Up & BANK1 & 4 & $1.46 \mathrm{E}-04$ & 22828 & 0.217336 & 0 \\
\hline Up & HIF3A & 3 & $6.24 \mathrm{E}-04$ & 67108 & 0.19472 & 0 \\
\hline Up & CNTNAP3 & 33 & $1.34 \mathrm{E}-02$ & 1433542 & 0.235939 & 0 \\
\hline Up & CCNJL & 39 & $1.50 \mathrm{E}-02$ & 3448762 & 0.260533 & 0 \\
\hline Up & ADAM32 & 21 & $6.74 \mathrm{E}-03$ & 1279704 & 0.223185 & 0 \\
\hline Up & PHYHD1 & 3 & 7.04E-04 & 88272 & 0.214905 & 0 \\
\hline Up & LAMA1 & 31 & $1.32 \mathrm{E}-02$ & 3129918 & 0.248298 & 0 \\
\hline Up & DNAI2 & 12 & $3.01 \mathrm{E}-03$ & 467486 & 0.2 & 0 \\
\hline Down & TRIM24 & 66 & $2.39 \mathrm{E}-02$ & 7566812 & 0.292625 & 0 \\
\hline Down & KLHL13 & 30 & $8.55 \mathrm{E}-03$ & 2607752 & 0.279499 & 0 \\
\hline Down & MAGEB2 & 46 & $1.08 \mathrm{E}-02$ & 4326748 & 0.268579 & 0 \\
\hline Down & РPBP & 22 & $7.30 \mathrm{E}-03$ & 2718118 & 0.22063 & 0 \\
\hline Down & TTK & 77 & $3.23 \mathrm{E}-02$ & 12267556 & 0.31332 & $7.21 \mathrm{E}-04$ \\
\hline Down & FAM71D & 3 & $6.39 \mathrm{E}-05$ & 15292 & 0.197561 & 0 \\
\hline Down & TOP2A & 102 & 4.15E-02 & 13126864 & 0.323325 & 0.002912 \\
\hline Down & KIF18A & 27 & $9.22 \mathrm{E}-03$ & 1047074 & 0.272947 & 0.002849 \\
\hline Down & TPX2 & 45 & $1.33 \mathrm{E}-02$ & 5212220 & 0.283324 & 0.00303 \\
\hline Down & POC1A & 3 & $2.10 \mathrm{E}-04$ & 38988 & 0.263713 & 0 \\
\hline Down & ARHGAP29 & 14 & $2.99 \mathrm{E}-03$ & 1435976 & 0.254092 & 0 \\
\hline Down & NRG1 & 66 & $2.98 \mathrm{E}-02$ & 8652224 & 0.277873 & 0 \\
\hline Down & PLCD4 & 3 & $1.07 \mathrm{E}-03$ & 235394 & 0.224878 & 0 \\
\hline Down & CADPS2 & 15 & 4.01E-03 & 1430040 & 0.22412 & 0 \\
\hline Down & $\mathrm{CCNF}$ & 13 & $2.36 \mathrm{E}-03$ & 321772 & 0.266733 & 0 \\
\hline Down & GTSE1 & 141 & $5.29 \mathrm{E}-02$ & 7085030 & 0.337622 & 0.008713 \\
\hline Down & TRHDE & 15 & 4.61E-03 & 1973410 & 0.23257 & 0 \\
\hline Down & SOX4 & 27 & $1.24 \mathrm{E}-02$ & 7096428 & 0.258212 & 0 \\
\hline Down & DLEU1 & 21 & $6.78 \mathrm{E}-03$ & 2289500 & 0.253469 & 0 \\
\hline Down & PLK1 & 276 & $1.64 \mathrm{E}-01$ & 39488508 & 0.35244 & 0.001016 \\
\hline Down & HAUS6 & 53 & $1.32 \mathrm{E}-02$ & 7866150 & 0.288256 & 0 \\
\hline Down & BUB1 & 95 & 4.02E-02 & 4659062 & 0.318162 & 0.008649 \\
\hline Down & SERINC2 & 8 & $2.18 \mathrm{E}-03$ & 1257316 & 0.206187 & 0 \\
\hline Down & KIF14 & 62 & $1.75 \mathrm{E}-02$ & 10376292 & 0.284688 & 0 \\
\hline Down & VWA1 & 14 & $4.26 \mathrm{E}-03$ & 366250 & 0.21001 & 0 \\
\hline Down & PTTG2 & 1 & $0.00 \mathrm{E}+00$ & 0 & 0.224824 & 0 \\
\hline Down & SEL1L & 58 & $2.60 \mathrm{E}-02$ & 9557720 & 0.270569 & 0 \\
\hline Down & NUSAP1 & 35 & $1.32 \mathrm{E}-02$ & 2253376 & 0.300363 & 0.008913 \\
\hline Down & CELA3A & 12 & $3.00 \mathrm{E}-03$ & 434718 & 0.234812 & 0 \\
\hline Down & DOK6 & 6 & 7.47E-04 & 300368 & 0.234664 & 0 \\
\hline Down & NRG1 & 66 & $2.98 \mathrm{E}-02$ & 8652224 & 0.277873 & 0 \\
\hline Down & DHX15 & 208 & $9.39 \mathrm{E}-02$ & 39966314 & 0.320847 & 0 \\
\hline Down & NEK2 & 52 & $1.53 \mathrm{E}-02$ & 5682386 & 0.277397 & 0 \\
\hline Down & TAS2R7 & 14 & $5.78 \mathrm{E}-03$ & 635150 & 0.247921 & 0.010989 \\
\hline Down & SPC24 & 41 & $1.22 \mathrm{E}-02$ & 1425412 & 0.291526 & 0.039024 \\
\hline
\end{tabular}




\begin{tabular}{|c|c|c|c|c|c|c|}
\hline Down & HMMR & 44 & $1.23 \mathrm{E}-02$ & 3764124 & 0.283002 & 0.003171 \\
\hline Down & PTP4A1 & 53 & $2.21 \mathrm{E}-02$ & 5500792 & 0.256435 & 0 \\
\hline Down & DAZAP2 & 99 & $4.35 \mathrm{E}-02$ & 9779248 & 0.28839 & 0.003651 \\
\hline Down & CDCA5 & 55 & $1.97 \mathrm{E}-02$ & 1719302 & 0.3 & 0.002903 \\
\hline Down & ANTXR2 & 12 & $3.86 \mathrm{E}-03$ & 876552 & 0.218842 & 0 \\
\hline Down & FBXL5 & 41 & $1.34 \mathrm{E}-02$ & 1287978 & 0.299253 & 0.006748 \\
\hline Down & PLA2G2D & 1 & $0.00 \mathrm{E}+00$ & 0 & 0.206084 & 0 \\
\hline Down & DAB1 & 67 & $2.85 \mathrm{E}-02$ & 5666990 & 0.279457 & 0.007689 \\
\hline Down & RBPJL & 2 & 5.37E-04 & 117698 & 0.224851 & 0 \\
\hline Down & FANCA & 86 & $2.87 \mathrm{E}-02$ & 13664846 & 0.284167 & 0 \\
\hline Down & NLGN1 & 11 & $3.82 \mathrm{E}-03$ & 881670 & 0.215525 & 0 \\
\hline Down & POU3F2 & 10 & $2.82 \mathrm{E}-03$ & 567692 & 0.225258 & 0 \\
\hline Down & PPP4R4 & 3 & $5.68 \mathrm{E}-04$ & 157816 & 0.205041 & 0 \\
\hline Down & GPR6 & 2 & $5.37 \mathrm{E}-04$ & 60688 & 0.210938 & 0 \\
\hline Down & PAX4 & 3 & $1.07 \mathrm{E}-03$ & 234714 & 0.21372 & 0 \\
\hline Down & ASXL2 & 18 & $6.05 \mathrm{E}-03$ & 1686090 & 0.242263 & 0 \\
\hline Down & IGFL3 & 1 & $0.00 \mathrm{E}+00$ & 0 & 1 & 0 \\
\hline Down & GRIK2 & 13 & 4.44E-03 & 430606 & 0.243976 & 0.025641 \\
\hline Down & NCAPG & 52 & $1.62 \mathrm{E}-02$ & 5737472 & 0.285517 & 0 \\
\hline Down & SLC19A1 & 3 & $5.43 \mathrm{E}-04$ & 132382 & 0.188974 & 0 \\
\hline Down & EPHB1 & 14 & $3.21 \mathrm{E}-03$ & 1135208 & 0.242942 & 0 \\
\hline Down & INHBE & 15 & 7.30E-03 & 1600282 & 0.257445 & 0 \\
\hline Down & FIBCD1 & 1 & $0.00 \mathrm{E}+00$ & 0 & 1 & 0 \\
\hline Down & RGS5 & 7 & $2.09 \mathrm{E}-03$ & 462166 & 0.237446 & 0 \\
\hline Down & CCNB2 & 36 & $8.70 \mathrm{E}-03$ & 3365996 & 0.275673 & 0.009524 \\
\hline Down & ADAMTSL1 & 7 & $2.82 \mathrm{E}-03$ & 352088 & 0.208506 & 0 \\
\hline Down & FAM83A & 7 & $1.40 \mathrm{E}-03$ & 115518 & 0.279038 & 0.047619 \\
\hline Down & LRFN1 & 20 & $9.73 \mathrm{E}-03$ & 1618782 & 0.237416 & 0 \\
\hline Down & PKLR & 19 & 4.89E-03 & 591642 & 0.267308 & 0.005848 \\
\hline Down & SLC25A15 & 15 & $5.26 \mathrm{E}-03$ & 1022014 & 0.258069 & 0 \\
\hline Down & PRC1 & 29 & $6.84 \mathrm{E}-03$ & 1142838 & 0.294452 & 0.031339 \\
\hline Down & AMIGO2 & 6 & $1.08 \mathrm{E}-03$ & 410596 & 0.178235 & 0 \\
\hline Down & KIF11 & 134 & $5.97 \mathrm{E}-02$ & 17181634 & 0.31282 & 0 \\
\hline Down & G2E3 & 6 & $1.27 \mathrm{E}-03$ & 455226 & 0.218061 & 0 \\
\hline Down & OR6T1 & 1 & $0.00 \mathrm{E}+00$ & 0 & 0.225613 & 0 \\
\hline Down & POLQ & 1 & $0.00 \mathrm{E}+00$ & 0 & 1 & 0 \\
\hline Down & GRIK2 & 13 & $4.44 \mathrm{E}-03$ & 430606 & 0.243976 & 0.025641 \\
\hline Down & RASSF3 & 28 & $7.78 \mathrm{E}-03$ & 3540288 & 0.258176 & 0 \\
\hline Down & PBK & 107 & $4.20 \mathrm{E}-02$ & 12464178 & 0.300775 & 0 \\
\hline Down & CENPA & 88 & 3.07E-02 & 4765456 & 0.300799 & 0.006293 \\
\hline Down & LYNX1 & 1 & $0.00 \mathrm{E}+00$ & 0 & 0.189426 & 0 \\
\hline Down & ASPDH & 10 & $3.69 \mathrm{E}-03$ & 974500 & 0.235242 & 0 \\
\hline Down & DLGAP5 & 23 & $7.53 \mathrm{E}-03$ & 2047406 & 0.302705 & 0.019763 \\
\hline
\end{tabular}




\begin{tabular}{|c|c|c|c|c|c|c|}
\hline Down & KIF20A & 38 & $9.99 \mathrm{E}-03$ & 3761204 & 0.277108 & 0 \\
\hline Down & CNKSR2 & 10 & $2.74 \mathrm{E}-03$ & 698616 & 0.245083 & 0 \\
\hline Down & GOLGA6A & 1 & $0.00 \mathrm{E}+00$ & 0 & 0.194204 & 0 \\
\hline Down & B4GALT6 & 4 & $1.00 \mathrm{E}+00$ & 12 & 1 & 0 \\
\hline Down & KRT28 & 1 & $0.00 \mathrm{E}+00$ & 0 & 1 & 0 \\
\hline Down & CDKN3 & 29 & $1.03 \mathrm{E}-02$ & 1383840 & 0.274414 & 0.002849 \\
\hline Down & DCAF4L2 & 7 & $1.14 \mathrm{E}-03$ & 263466 & 0.229603 & 0 \\
\hline Down & CALHM1 & 2 & $0.00 \mathrm{E}+00$ & 0 & 0 & 0 \\
\hline Down & ADD2 & 17 & $5.29 \mathrm{E}-03$ & 1384972 & 0.251723 & 0 \\
\hline Down & CDKN3 & 29 & $1.03 \mathrm{E}-02$ & 1383840 & 0.274414 & 0.002849 \\
\hline Down & RYR2 & 28 & $8.08 \mathrm{E}-03$ & 2614302 & 0.258642 & 0 \\
\hline Down & C20orf203 & 2 & $1.00 \mathrm{E}+00$ & 2 & 1 & 0 \\
\hline Down & YTHDF1 & 61 & $2.13 \mathrm{E}-02$ & 7937228 & 0.298366 & 0 \\
\hline Down & FOXM1 & 80 & $2.74 \mathrm{E}-02$ & 8443400 & 0.298749 & 0.002331 \\
\hline Down & GJA4 & 3 & $1.07 \mathrm{E}-03$ & 514922 & 0.181296 & 0 \\
\hline Down & PF4 & 11 & $5.89 \mathrm{E}-03$ & 2016954 & 0.188248 & 0.027778 \\
\hline Down & ANKFY1 & 41 & $1.24 \mathrm{E}-02$ & 5747558 & 0.26362 & 0 \\
\hline Down & AKAP3 & 10 & $3.76 \mathrm{E}-03$ & 892188 & 0.240046 & 0 \\
\hline Down & DCAF12L2 & 11 & $3.30 \mathrm{E}-03$ & 771012 & 0.231472 & 0 \\
\hline Down & BDNF & 11 & 4.67E-03 & 1264752 & 0.242373 & 0 \\
\hline Down & BIRC5 & 61 & $2.10 \mathrm{E}-02$ & 6990168 & 0.290549 & 0 \\
\hline Down & SLC39A5 & 45 & $1.94 \mathrm{E}-02$ & 2952184 & 0.228407 & 0 \\
\hline Down & GLYATL3 & 1 & $0.00 \mathrm{E}+00$ & 0 & 0.190715 & 0 \\
\hline Down & L1CAM & 24 & $6.90 \mathrm{E}-03$ & 2825930 & 0.267884 & 0 \\
\hline Down & SV2C & 7 & $2.26 \mathrm{E}-03$ & 545018 & 0.20922 & 0 \\
\hline Down & KIF15 & 27 & $7.88 \mathrm{E}-03$ & 1833408 & 0.267865 & 0 \\
\hline Down & FANCD2 & 74 & $3.14 \mathrm{E}-02$ & 6501308 & 0.312978 & 0.004813 \\
\hline Down & RACGAP1 & 89 & $3.18 \mathrm{E}-02$ & 7802394 & 0.304263 & 0.005107 \\
\hline Down & FAM20C & 12 & 4.87E-03 & 828322 & 0.200852 & 0 \\
\hline Down & NUF2 & 44 & $1.42 \mathrm{E}-02$ & 2296298 & 0.301725 & $3.38 \mathrm{E}-02$ \\
\hline Down & QSOX1 & 15 & $4.90 \mathrm{E}-03$ & 1223750 & 0.241087 & 0 \\
\hline Down & SLITRK6 & 1 & $0.00 \mathrm{E}+00$ & 0 & 0.206759 & 0 \\
\hline Down & FAM91A1 & 23 & 7.07E-03 & 2542312 & 0.2639 & 0 \\
\hline Down & GPR161 & 16 & $8.15 \mathrm{E}-03$ & 864880 & 0.246363 & 0.008333 \\
\hline Down & SCEL & 14 & $5.37 \mathrm{E}-03$ & 1611186 & 0.249883 & 0 \\
\hline Down & CXCR3 & 6 & $2.15 \mathrm{E}-03$ & 733476 & 0.158492 & 0.066667 \\
\hline Down & RFT1 & 21 & $6.86 \mathrm{E}-03$ & 2273166 & 0.225517 & 0 \\
\hline Down & PSG5 & 4 & $1.24 \mathrm{E}-03$ & 315510 & 0.199775 & 0 \\
\hline Down & RAD51 & 120 & 4.54E-02 & 8161594 & 0.307324 & 0.001883 \\
\hline Down & ADAMTSL1 & 7 & $2.82 \mathrm{E}-03$ & 352088 & 0.208506 & 0 \\
\hline Down & HMGB4 & 3 & $6.06 \mathrm{E}-04$ & 146328 & 0.226988 & 0 \\
\hline Down & TROAP & 33 & $1.24 \mathrm{E}-02$ & 1251972 & 0.293872 & 0.006452 \\
\hline Down & RASSF10 & 38 & $1.21 \mathrm{E}-02$ & 3884098 & 0.259851 & 0 \\
\hline
\end{tabular}




\begin{tabular}{|c|c|c|c|c|c|c|}
\hline Down & ZC3H12D & 1 & $0.00 \mathrm{E}+00$ & 0 & 1 & 0 \\
\hline Down & HJURP & 21 & $4.31 \mathrm{E}-03$ & 873270 & 0.271041 & 0.035088 \\
\hline Down & XKR6 & 1 & $0.00 \mathrm{E}+00$ & 0 & 0.225613 & 0 \\
\hline Down & UNC5D & 1 & $0.00 \mathrm{E}+00$ & 0 & 0.201809 & 0 \\
\hline Down & C1orf189 & 2 & $1.00 \mathrm{E}+00$ & 2 & 1 & 0 \\
\hline Down & PSTPIP2 & 8 & $1.87 \mathrm{E}-03$ & 472358 & 0.247427 & 0 \\
\hline Down & XKR6 & 1 & $0.00 \mathrm{E}+00$ & 0 & 0.225613 & 0 \\
\hline Down & EXD1 & 1 & $0.00 \mathrm{E}+00$ & 0 & 1 & 0 \\
\hline Down & KIF21B & 30 & $9.70 \mathrm{E}-03$ & 2431650 & 0.256842 & 0 \\
\hline Down & GRIP1 & 53 & $1.84 \mathrm{E}-02$ & 6233398 & 0.270372 & 0.001569 \\
\hline Down & POU3F3 & 3 & $5.62 \mathrm{E}-04$ & 84710 & 0.208378 & 0 \\
\hline Down & CPN1 & 6 & $1.71 \mathrm{E}-03$ & 328228 & 0.22172 & 0 \\
\hline Down & SPC25 & 35 & $6.24 \mathrm{E}-03$ & 630312 & 0.27207 & 0.052101 \\
\hline Down & C12ORF66 & 4 & $1.61 \mathrm{E}-03$ & 77742 & 0.201711 & 0 \\
\hline Down & MELK & 23 & $6.63 \mathrm{E}-03$ & 1668988 & 0.250673 & 0 \\
\hline Down & TLE4 & 28 & $9.56 \mathrm{E}-03$ & 2438214 & 0.252507 & 0 \\
\hline Down & MND1 & 7 & $2.70 \mathrm{E}-03$ & 788252 & 0.224986 & 0 \\
\hline Down & ZMAT3 & 24 & 7.69E-03 & 2968830 & 0.271931 & 0 \\
\hline Down & ATP2A3 & 53 & $2.39 \mathrm{E}-02$ & 6619182 & 0.256171 & 0 \\
\hline Down & GRIP1 & 53 & $1.84 \mathrm{E}-02$ & 6233398 & 0.270372 & 0.001569 \\
\hline Down & CENPM & 15 & $2.31 \mathrm{E}-03$ & 174586 & 0.25524 & 0.114286 \\
\hline Down & GRIK2 & 13 & 4.44E-03 & 430606 & 0.243976 & 0.025641 \\
\hline Down & ZSCAN2 & 1 & $0.00 \mathrm{E}+00$ & 0 & 1 & 0 \\
\hline Down & GPR26 & 1 & $0.00 \mathrm{E}+00$ & 0 & 1 & 0 \\
\hline Down & NXPH4 & 4 & $1.61 \mathrm{E}-03$ & 1133406 & 0.17246 & 0 \\
\hline Down & UNC5D & 1 & $0.00 \mathrm{E}+00$ & 0 & 0.201809 & 0 \\
\hline Down & RNF215 & 1 & $0.00 \mathrm{E}+00$ & 0 & 1 & 0 \\
\hline Down & STMN1 & 65 & $2.51 \mathrm{E}-02$ & 7293058 & 0.286065 & $4.81 \mathrm{E}-04$ \\
\hline Down & ARHGAP20 & 2 & $1.00 \mathrm{E}-05$ & 3872 & 0.219564 & 0 \\
\hline Down & FAM72D & 1 & $0.00 \mathrm{E}+00$ & 0 & 0.225613 & 0 \\
\hline Down & HTR3D & 4 & 7.06E-04 & 153078 & 0.180646 & 0 \\
\hline Down & ATP2B2 & 118 & $5.57 \mathrm{E}-02$ & 10270314 & 0.275124 & $2.90 \mathrm{E}-04$ \\
\hline Down & CCNG2 & 11 & $3.40 \mathrm{E}-03$ & 744714 & 0.210806 & 0 \\
\hline Down & $\mathrm{RCC} 1$ & 102 & $3.87 \mathrm{E}-02$ & 10546556 & 0.314165 & 0.001941 \\
\hline Down & NPVF & 3 & $3.46 \mathrm{E}-05$ & 17228 & 0.217653 & 0 \\
\hline Down & KIF23 & 62 & $2.33 \mathrm{E}-02$ & 4491558 & 0.306515 & 0.015819 \\
\hline Down & PSG11 & 4 & $1.12 \mathrm{E}-03$ & 136444 & 0.188324 & 0 \\
\hline Down & PRR11 & 95 & $3.70 \mathrm{E}-02$ & 16341014 & 0.259923 & 0 \\
\hline Down & LCE1B & 22 & $1.04 \mathrm{E}-02$ & 1365886 & 0.209408 & 0 \\
\hline Down & CCNA1 & 83 & $2.81 \mathrm{E}-02$ & 8701494 & 0.2972 & $2.47 \mathrm{E}-03$ \\
\hline Down & TLE4 & 28 & $9.56 \mathrm{E}-03$ & 2438214 & 0.252507 & 0 \\
\hline Down & HIGD1B & 3 & $1.00 \mathrm{E}+00$ & 6 & 1 & 0 \\
\hline Down & OXR1 & 10 & $1.57 \mathrm{E}-03$ & 396854 & 0.225914 & 0 \\
\hline
\end{tabular}




\begin{tabular}{|c|c|c|c|c|c|c|}
\hline Down & DEFA3 & 1 & $0.00 \mathrm{E}+00$ & 0 & 0.184876 & 0 \\
\hline Down & DISC1 & 138 & $5.45 \mathrm{E}-02$ & 23062588 & 0.288949 & 0 \\
\hline Down & CLTC & 333 & $1.73 \mathrm{E}-01$ & 59969246 & 0.345833 & 0.001483 \\
\hline Down & MTBP & 7 & $9.00 \mathrm{E}-04$ & 308238 & 0.25191 & 0 \\
\hline Down & COLEC10 & 14 & $5.78 \mathrm{E}-03$ & 496564 & 0.230569 & 0 \\
\hline Down & PNPLA7 & 1 & $0.00 \mathrm{E}+00$ & 0 & 1 & 0 \\
\hline Down & TLE4 & 28 & $9.56 \mathrm{E}-03$ & 2438214 & 0.252507 & 0 \\
\hline Down & MYO1D & 58 & $2.01 \mathrm{E}-02$ & 6943106 & 0.297723 & 0 \\
\hline Down & CXCR2 & 9 & $2.70 \mathrm{E}-03$ & 571596 & 0.206862 & 0 \\
\hline Down & POU3F4 & 3 & $3.10 \mathrm{E}-04$ & 10712 & 0.214335 & 0 \\
\hline Down & KIF26A & 3 & $5.58 \mathrm{E}-04$ & 216568 & 0.213757 & 0 \\
\hline Down & VWF & 20 & $6.90 \mathrm{E}-03$ & 3379006 & 0.226877 & 0 \\
\hline Down & ZSCAN12 & 40 & $1.72 \mathrm{E}-02$ & 4511560 & 0.249014 & 0 \\
\hline Down & OMP & 1 & $0.00 \mathrm{E}+00$ & 0 & 1 & 0 \\
\hline Down & SPINK5 & 1 & $0.00 \mathrm{E}+00$ & 0 & 0.200236 & 0 \\
\hline Down & MYBPC1 & 24 & $1.02 \mathrm{E}-02$ & 2208902 & 0.223047 & 0 \\
\hline Down & RFC3 & 48 & $1.19 \mathrm{E}-02$ & 6744340 & 0.286615 & 0 \\
\hline Down & KIAA1958 & 14 & $4.06 \mathrm{E}-03$ & 1021098 & 0.246641 & 0 \\
\hline Down & TMEM40 & 1 & $0.00 \mathrm{E}+00$ & 0 & 1 & 0 \\
\hline Down & CTAG1A & 2 & $5.37 \mathrm{E}-04$ & 160340 & 0.182031 & 0 \\
\hline Down & CHRNA7 & 5 & $1.20 \mathrm{E}-03$ & 315000 & 0.238785 & 0 \\
\hline Down & PARD6G & 21 & $6.07 \mathrm{E}-03$ & 2092448 & 0.251349 & 0 \\
\hline Down & ZNF160 & 4 & $1.00 \mathrm{E}+00$ & 12 & 1 & 0 \\
\hline Down & CCL3L3 & 1 & $1.00 \mathrm{E}+00$ & 6 & 1 & 0 \\
\hline Down & CKS2 & 36 & $8.66 \mathrm{E}-03$ & 1293372 & 0.279227 & $2.50 \mathrm{E}-02$ \\
\hline Down & HSD3B2 & 19 & $6.19 \mathrm{E}-03$ & 434014 & 0.258678 & 0.005848 \\
\hline Down & RAD21L1 & 1 & $0.00 \mathrm{E}+00$ & 0 & 0.198223 & 0 \\
\hline Down & HS3ST2 & 11 & $4.28 \mathrm{E}-03$ & 1134682 & 0.226849 & 0 \\
\hline Down & GRM5 & 10 & $2.07 \mathrm{E}-03$ & 756604 & 0.225846 & 0 \\
\hline Down & SPEF2 & 3 & $1.07 \mathrm{E}-03$ & 311542 & 0.166704 & 0 \\
\hline Down & KIR3DX1 & 3 & 5.37E-04 & 151612 & 0.229872 & 0 \\
\hline Down & EYS & 1 & $0.00 \mathrm{E}+00$ & 0 & 0.217653 & 0 \\
\hline Down & CENPH & 38 & $1.29 \mathrm{E}-02$ & 1191544 & 0.293478 & 0.022222 \\
\hline Down & C3orf52 & 11 & $3.74 \mathrm{E}-03$ & 897310 & 0.232454 & 0 \\
\hline Down & UNC13A & 7 & $2.18 \mathrm{E}-03$ & 1007070 & 0.215862 & 0 \\
\hline Down & RUFY4 & 3 & $5.58 \mathrm{E}-04$ & 112984 & 0.219927 & 0 \\
\hline Down & GPR17 & 1 & $0.00 \mathrm{E}+00$ & 0 & 1 & 0 \\
\hline Down & RAD21L1 & 1 & $0.00 \mathrm{E}+00$ & 0 & 0.198223 & 0 \\
\hline Down & CASK & 79 & $3.54 \mathrm{E}-02$ & 8491074 & 0.295926 & 0 \\
\hline Down & OXR1 & 10 & $1.57 \mathrm{E}-03$ & 396854 & 0.225914 & 0 \\
\hline
\end{tabular}

Table 7 miRNA - target gene interaction table 


\begin{tabular}{|c|c|c|c|c|c|c|c|}
\hline Regulation & $\begin{array}{l}\text { target } \\
\text { Genes }\end{array}$ & Degree & Top MicroRNA & Regulation & $\begin{array}{l}\text { target } \\
\text { Genes }\end{array}$ & Degree & Top MicroRNA \\
\hline Up & SALL1 & 53 & hsa-mir-548b-3p & Down & TRIM24 & 21 & hsa-mir-4477a \\
\hline \multirow[t]{2}{*}{ Up } & & & & Down & & & \\
\hline & NKX2-2 & 20 & hsa-mir-6829-3p & & KLHL13 & 1 & hsa-mir-218-5p \\
\hline \multirow[t]{2}{*}{ Up } & & & & Down & & & \\
\hline & PTCHD1 & 60 & hsa-mir-548a-3p & & MAGEB2 & 2 & hsa-mir-98-5p \\
\hline \multirow[t]{2}{*}{ Up } & & & & Down & & & \\
\hline & BMP7 & 22 & hsa-mir-6890-3p & & C3orf80 & 10 & hsa-mir-3153 \\
\hline \multirow[t]{2}{*}{ Up } & & & & Down & & & \\
\hline & TMEM154 & 49 & hsa-mir-3187-3p & & PPBP & 1 & hsa-mir-335-5p \\
\hline \multirow[t]{2}{*}{ Up } & & & & Down & & & \\
\hline & SERTM1 & 13 & hsa-mir-3924 & & TTK & 7 & hsa-mir-376a-3p \\
\hline \multirow[t]{2}{*}{ Up } & & & & Down & & & \\
\hline & FAM83F & 94 & hsa-mir-612 & & TOP2A & 17 & hsa-mir-708-5p \\
\hline \multirow[t]{2}{*}{ Up } & & & & Down & & & \\
\hline & SGCD & 41 & hsa-mir-4761-5p & & KIF18A & 2 & hsa-mir-192-5p \\
\hline \multirow[t]{2}{*}{ Up } & & & & Down & & & \\
\hline & C1orf168 & 2 & hsa-mir-148b-3p & & TPX2 & 1 & hsa-mir-193b-3p \\
\hline \multirow[t]{2}{*}{ Up } & & & & Down & & & \\
\hline & GRHL2 & 1 & hsa-mir-26b-5p & & POC1A & 69 & hsa-mir-3605-5p \\
\hline \multirow[t]{2}{*}{ Up } & & & & Down & & & \\
\hline & SMR3B & 1 & hsa-mir-495-3p & & ARHGAP29 & 25 & hsa-mir-6814-5p \\
\hline \multirow[t]{2}{*}{ Up } & & & & Down & & & \\
\hline & SCRG1 & 33 & hsa-mir-1304-3p & & NRG1 & 1 & hsa-mir-124-3p \\
\hline \multirow[t]{2}{*}{ Up } & & & & Down & & & \\
\hline & IL20RA & 1 & hsa-mir-26b-5p & & CADPS2 & 4 & hsa-mir-30c-5p \\
\hline \multirow[t]{2}{*}{ Up } & & & & Down & & & \\
\hline & FCN2 & 8 & hsa-mir-8064 & & ACER2 & 46 & hsa-let-7d-5p \\
\hline \multirow[t]{2}{*}{ Up } & & & & Down & & & \\
\hline & DPPA4 & 1 & hsa-mir-26b-5p & & CCNF & 102 & hsa-mir-324-3p \\
\hline \multirow[t]{2}{*}{ Up } & & & & Down & & & \\
\hline & SPTLC3 & 32 & hsa-mir-206 & & GTSE1 & 2 & hsa-mir-484 \\
\hline \multirow[t]{2}{*}{ Up } & & & & Down & & & \\
\hline & ATP4A & 1 & hsa-mir-1289 & & SOX4 & 160 & hsa-mir-519a-3p \\
\hline \multirow[t]{2}{*}{ Up } & & & & Down & & & \\
\hline & SDK2 & 33 & hsa-mir-644a & & DLEU1 & 31 & hsa-mir-616-5p \\
\hline \multirow[t]{2}{*}{ Up } & & & & Down & & & \\
\hline & RSPO3 & 1 & hsa-mir-335-5p & & PLK1 & 17 & hsa-mir-3120-3p \\
\hline \multirow[t]{2}{*}{ Up } & & & & Down & & & \\
\hline & FGFR2 & 5 & hsa-mir-19b-1-5p & & HAUS6 & 14 & hsa-mir-5582-3p \\
\hline \multirow[t]{2}{*}{ Up } & & & & Down & & & \\
\hline & IQCA1 & 1 & hsa-mir-193b-3p & & BUB1 & 67 & hsa-mir-497-3p \\
\hline \multirow[t]{2}{*}{ Up } & & & & Down & & & \\
\hline & MGST1 & 2 & hsa-mir-652-3p & & SERINC2 & 1 & hsa-mir-335-5p \\
\hline Up & & & & Down & & & \\
\hline & FABP7 & 7 & hsa-mir-6823-3p & & KIF14 & 14 & hsa-mir-3133 \\
\hline Up & & & & Down & & & \\
\hline & PON3 & 1 & hsa-mir-335-5p & & VWA1 & 30 & hsa-mir-592 \\
\hline
\end{tabular}


Up

GAL $\quad 8 \quad$ hsa-mir-4671-5p

Up

PAX6

23

hsa-mir-599

Up

Up

Up

Up

Up

Up

Up

Up

Up

Up

Up

Up

Up

Up

Up

Up

Up

Up

Up

Up

Up

Up

Up

SYPL2 1 hsa-mir-615-3p

SLC15A1 $19 \quad$ hsa-mir-7977

TMPRSS3 2 hsa-mir-204-5p

ROR2 3 hsa-mir-451a

DACT2 2

SMTNL2

43

FBN2

16

NTF4

1

SRL

SLIT1

33

BBOX1

4

hsa-mir-4477a

ART3

2

hsa-mir-122-5p

NRK 1 hsa-mir-335-5p

ID4 $91 \quad$ hsa-mir-4311

PLIN4 1 hsa-mir-335-5p

TRDN 17 hsa-mir-501-3p

CLIC5 54 hsa-mir-4480

BTBD17 1 hsa-mir-335-5p

CCKBR 3 hsa-mir-148a-3p

S100A1 4 hsa-mir-138-5p

CBLN2 6 hsa-mir-6819-3p

PMAIP1 128 hsa-mir-411-3p

XDH 1 hsa-mir-26b-5p
Down

FRMPD3 $18 \quad$ hsa-mir-6511a-3p

Down

SEL1L $\quad 12$ hsa-mir-548as-3p

Down

NUSAP1 11 hsa-let-7b-5p

Down

DOK6 23 hsa-mir-3679-3p

Down

NRG1 1 hsa-mir-124-3p

Down

DHX15 7 hsa-mir-221-3p

Down

NEK2 2 hsa-mir-92a-3p

Down

TAS2R7 1 hsa-mir-142-3p

Down

SPC24 $26 \quad$ hsa-mir-3664-5p

Down

HMMR 5 hsa-mir-106b-5p

Down

PTP4A1 132 hsa-mir-186-5p

Down

DAZAP2 109 hsa-mir-511-5p

Down

Down

CDCA5 4 hsa-mir-331-3p

Down

ANTXR2 $60 \quad$ hsa-mir-3065-5p

Down

FBXL5 $31 \quad$ hsa-mir-4731-3p

PLA2G2D 20 hsa-mir-548au-3p

\section{Down}

Down

RBPJL $25 \quad$ hsa-mir-6731-5p

Down

CENPI 2 hsa-mir-192-5p

Down

NLGN1 8 hsa-mir-670-3p

Down

POU3F2 19 hsa-mir-6781-5p

PAX4 8 hsa-mir-4783-5p

Down

ASXL2 $48 \quad$ hsa-mir-5002-5p

Down

Down

Down

GRIK2 1 hsa-mir-26b-5p

NCAPG $20 \quad$ hsa-mir-664b-3p

SLC19A1 9 hsa-mir-4422 


\begin{tabular}{|c|c|c|c|c|c|c|c|}
\hline Up & & & & Down & & & \\
\hline & TRIM29 & 18 & hsa-mir-1268a & & EPHB1 & 18 & hsa-mir-548aw \\
\hline Up & & & & Down & & & \\
\hline & UGT2A2 & 9 & hsa-mir-548u & & INHBE & 2 & hsa-mir-98-5p \\
\hline Up & & & & Down & & & \\
\hline & SOX5 & 38 & hsa-mir-555 & & FIBCD1 & 12 & hsa-mir-6717-5p \\
\hline Up & & & & Down & & & \\
\hline & S100A1 & 4 & hsa-mir-132-3p & & RGS5 & 42 & hsa-mir-4255 \\
\hline Up & & & & Down & & & \\
\hline & GALR1 & 8 & hsa-mir-130b-5p & & GUCA2B & 1 & hsa-mir-335-5p \\
\hline Up & & & & Down & & & \\
\hline & GAS1 & 33 & hsa-mir-449b-5p & & CCNB2 & 6 & hsa-let-7f-5p \\
\hline Up & & & & Down & & & \\
\hline & PPP1R17 & 1 & hsa-mir-335-5p & & ADAMTSL1 & 2 & hsa-mir-25-3p \\
\hline Up & & & & Down & & & \\
\hline & IFNE & 16 & hsa-mir-4786-5p & & FAM83A & 5 & hsa-mir-5090 \\
\hline Up & & & & Down & & & \\
\hline & CELSR1 & 2 & hsa-mir-92a-3p & & LRFN1 & 18 & hsa-mir-6887-3p \\
\hline Up & & & & Down & & & \\
\hline & C4orf19 & 5 & hsa-mir-1273f & & SLC25A15 & 20 & hsa-mir-6720-3p \\
\hline Up & & & & Down & & & \\
\hline & POMC & 2 & hsa-mir-488-3p & & GABRQ & 1 & hsa-mir-335-5p \\
\hline Up & & & & Down & & & \\
\hline & SLC6A14 & 1 & hsa-mir-26b-5p & & PRC1 & 17 & hsa-mir-3977 \\
\hline Up & & & & Down & & & \\
\hline & LRAT & 47 & hsa-mir-4770 & & AMIGO2 & 2 & hsa-mir-155-5p \\
\hline Up & & & & Down & & & \\
\hline & SPTLC3 & 32 & hsa-mir-6514-5p & & $\mathrm{CDC} 45$ & 2 & hsa-mir-575 \\
\hline Up & & & & Down & & & \\
\hline & KLK11 & 1 & hsa-mir-335-5p & & PRDM11 & 1 & hsa-mir-335-5p \\
\hline Up & & & & Down & & & \\
\hline & F5 & 4 & hsa-mir-634 & & CYP27B1 & 12 & hsa-mir-1178-5p \\
\hline Up & & & & Down & & & \\
\hline & CNTNAP2 & 14 & hsa-mir-571 & & KIF11 & 15 & hsa-mir-4495 \\
\hline Up & & & & Down & & & \\
\hline & $\mathrm{PI} 16$ & 2 & hsa-mir-1-1 & & G2E3 & 54 & hsa-mir-520g-3p \\
\hline Up & & & & Down & & & \\
\hline & ENPP6 & 24 & hsa-mir-6077 & & POLQ & 86 & hsa-mir-664a-5p \\
\hline Up & & & & Down & & & \\
\hline & GBP6 & 24 & hsa-mir-548j-3p & & GRIK2 & 1 & hsa-mir-26b-5p \\
\hline Up & & & & Down & & & \\
\hline & NEURL3 & 1 & hsa-mir-335-5p & & RASSF3 & 3 & hsa-mir-20a-3p \\
\hline Up & & & & Down & & & \\
\hline & CLIC5 & 54 & hsa-mir-4301 & & PBK & 4 & hsa-mir-4699-3p \\
\hline Up & & & & Down & & & \\
\hline & RASL11B & 7 & hsa-mir-3137 & & CENPA & 23 & hsa-mir-4719 \\
\hline Up & & & & Down & & & \\
\hline & KCNJ12 & 24 & hsa-mir-770-5p & & LYNX1 & 1 & hsa-mir-744-5p \\
\hline Up & & & & Down & & & \\
\hline & AQP4 & 1 & hsa-mir-320a & & $\mathrm{EVX} 2$ & 9 & hsa-mir-33b-3p \\
\hline
\end{tabular}




\begin{tabular}{|c|c|c|c|c|c|c|c|}
\hline \multirow[t]{2}{*}{ Up } & \multicolumn{7}{|c|}{ Down } \\
\hline & P2RY2 & 16 & hsa-mir-4433b-3p & & KRTAP5-7 & 1 & hsa-mir-26b-5p \\
\hline \multirow[t]{2}{*}{ Up } & & & & Down & & & \\
\hline & IL22RA1 & 1 & hsa-mir-26b-5p & & DLGAP5 & 21 & hsa-mir-520e \\
\hline \multirow[t]{2}{*}{ Up } & & & & Down & & & \\
\hline & RNF207 & 10 & hsa-mir-3190-5p & & EDN1 & 57 & hsa-mir-379-5p \\
\hline \multirow[t]{2}{*}{ Up } & & & & Down & & & \\
\hline & ADAMTS18 & 16 & hsa-mir-588 & & KIF20A & 5 & hsa-mir-23a-3p \\
\hline \multirow[t]{2}{*}{ Up } & & & & Down & & & \\
\hline & NGFR & 23 & hsa-mir-6732-5p & & CNKSR2 & 10 & hsa-mir-6808-3p \\
\hline \multirow[t]{2}{*}{ Up } & & & & Down & & & \\
\hline & KCNJ16 & 1 & hsa-mir-148b-3p & & GOLGA6A & 4 & hsa-mir-450b-5p \\
\hline \multirow[t]{2}{*}{ Up } & & & & Down & & & \\
\hline & GPHA2 & 24 & hsa-mir-1180-5p & & B4GALT6 & 19 & hsa-mir-1248 \\
\hline \multirow[t]{2}{*}{ Up } & & & & Down & & & \\
\hline & C6orf132 & 48 & hsa-mir-4638-5p & & ARHGAP42 & 22 & hsa-mir-6131 \\
\hline \multirow[t]{2}{*}{ Up } & & & & Down & & & \\
\hline & PHACTR2 & 47 & hsa-mir-590-3p & & KRT28 & 1 & hsa-mir-1229-3p \\
\hline \multirow[t]{2}{*}{ Up } & & & & Down & & & \\
\hline & EVC2 & 18 & hsa-mir-6873-3p & & CDKN3 & 18 & hsa-mir-4695-3p \\
\hline \multirow[t]{2}{*}{ Up } & & & & Down & & & \\
\hline & FOXL2 & 10 & hsa-mir-1253 & & DCAF4L2 & 2 & hsa-mir-494-3p \\
\hline \multirow[t]{2}{*}{ Up } & & & & & & & \\
\hline & FAM 3B & 1 & hsa-mir-335-5p & & & & \\
\hline \multirow[t]{2}{*}{ Up } & & & & & & & \\
\hline & DLK1 & 1 & hsa-mir-15a-5p & & & & \\
\hline \multirow[t]{2}{*}{ Up } & & & & & & & \\
\hline & BLID & 1 & hsa-mir-335-5p & & & & \\
\hline \multirow[t]{2}{*}{ Up } & & & & & & & \\
\hline & UGT2A1 & 10 & hsa-mir-4762-5p & & & & \\
\hline \multirow[t]{2}{*}{ Up } & & & & & & & \\
\hline & OGN & 1 & hsa-mir-526b-5p & & & & \\
\hline \multirow[t]{2}{*}{ Up } & & & & & & & \\
\hline & $\mathrm{TF}$ & 2 & hsa-mir-19a-3p & & & & \\
\hline \multirow[t]{2}{*}{ Up } & & & & & & & \\
\hline & SMAD9 & 45 & hsa-mir-4419b & & & & \\
\hline \multirow[t]{2}{*}{ Up } & & & & & & & \\
\hline & LINC00598 & 209 & hsa-mir-890 & & & & \\
\hline \multirow[t]{2}{*}{ Up } & & & & & & & \\
\hline & SOX2 & 15 & hsa-mir-126-3p & & & & \\
\hline Up & & & & & & & \\
\hline & KLK12 & 2 & hsa-mir-1-3p & & & & \\
\hline Up & & & & & & & \\
\hline & KRT14 & 2 & hsa-mir-124-3p & & & & \\
\hline Up & & & & & & & \\
\hline & DLGAP2 & 8 & hsa-mir-20a-3p & & & & \\
\hline Up & & & & & & & \\
\hline & LRRC2 & 46 & hsa-mir-3914 & & & & \\
\hline Up & AMOT & 23 & hsa-mir-500a-5p & & & & \\
\hline
\end{tabular}




\begin{tabular}{|c|c|c|c|}
\hline $\mathrm{Jp}$ & LINC00598 & 209 & hsa-mir-518a-5p \\
\hline Up & HSD11B1 & 2 & hsa-mir-26b-5p \\
\hline Up & SGCD & 41 & hsa-mir-4698 \\
\hline Up & PRSS8 & 1 & hsa-mir-335-5p \\
\hline Up & C6orf118 & 2 & hsa-mir-1-1 \\
\hline Up & CRISP2 & 2 & hsa-mir-27a-3p \\
\hline Up & $\mathrm{IHH}$ & 22 & hsa-mir-6839-5p \\
\hline Up & MCOLN2 & 18 & hsa-mir-6731-3p \\
\hline Up & SLC39A12 & 1 & hsa-mir-335-5p \\
\hline up & VIT & 1 & hsa-mir-124-3p \\
\hline Up & PRRX2 & 1 & hsa-mir-124-3p \\
\hline Up & PPP1R17 & 1 & hsa-mir-335-5p \\
\hline Up & TSHB & 1 & hsa-mir-26b-5p \\
\hline Up & AGR2 & 2 & hsa-mir-197-3p \\
\hline Up & KBTBD12 & 39 & hsa-mir-4675 \\
\hline up & GADD45G & 1 & hsa-mir-181a-5p \\
\hline Up & LIX1 & 3 & hsa-mir-4668-5p \\
\hline Up & PRSS45 & 8 & hsa-mir-765 \\
\hline Up & MFAP4 & 1 & hsa-mir-124-3p \\
\hline Up & ESPNL & 1 & hsa-mir-375 \\
\hline Up & NFIX & 67 & hsa-mir-4488 \\
\hline Up & EVC2 & 18 & hsa-mir-1236-3p \\
\hline Up & COL8A2 & 2 & hsa-mir-124-3p \\
\hline Up & GNRHR & 4 & hsa-mir-1-3p \\
\hline 0 & SULT1C2 & 2 & hsa-mir-7-5p \\
\hline
\end{tabular}




\begin{tabular}{|c|c|c|c|}
\hline & CA8 & 54 & hsa-mir-646 \\
\hline Up & CHRDL1 & 54 & hsa-mir-583 \\
\hline Up & RPRM & 32 & hsa-mir-939-5p \\
\hline Up & OCA2 & 12 & hsa-mir-1299 \\
\hline Up & MPPED1 & 15 & hsa-mir-516a-3p \\
\hline Up & OGN & 1 & hsa-mir-526b-5p \\
\hline Up & GADD45G & 1 & hsa-mir-181a-5p \\
\hline Up & VSNL1 & 25 & hsa-mir-6873-5p \\
\hline Up & FGFR3 & 9 & hsa-mir-425-5p \\
\hline Up & WNT5A & 29 & hsa-mir-4680-3p \\
\hline Up & SFRP2 & 2 & hsa-mir-218-5p \\
\hline Up & HSPB3 & 1 & hsa-mir-335-5p \\
\hline Up & PAX6 & 23 & hsa-mir-223-3p \\
\hline Up & CYBRD1 & 42 & hsa-mir-4517 \\
\hline Up & PGR & 2 & hsa-mir-181a-5p \\
\hline Up & HSD17B2 & 2 & hsa-mir-124-3p \\
\hline Up & OPCML & 9 & hsa-mir-3149 \\
\hline Up & AGR3 & 1 & hsa-mir-335-5p \\
\hline Up & SLIT1 & 33 & hsa-mir-4726-5p \\
\hline Up & SOX9 & 30 & hsa-mir-1323 \\
\hline Up & CSHL1 & 1 & hsa-mir-16-5p \\
\hline UP & CHST9 & 2 & hsa-mir-92a-3p \\
\hline Up & NRK & 1 & hsa-mir-335-5p \\
\hline Up & SULT1C2 & 2 & hsa-mir-7-5p \\
\hline & SULT1C4 & 10 & hsa-mir-6833-3p \\
\hline
\end{tabular}




\begin{tabular}{|c|c|c|c|}
\hline & SNTG2 & 1 & hsa-mir-146a-5p \\
\hline \multicolumn{4}{|r|}{ 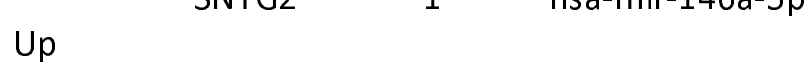 } \\
\hline \multicolumn{4}{|c|}{ Up } \\
\hline & AGMO & 23 & hsa-mir-1976 \\
\hline \multicolumn{4}{|l|}{ Up } \\
\hline & PTER & 2 & hsa-mir-130b-3p \\
\hline \multicolumn{4}{|r|}{ the } \\
\hline \multicolumn{4}{|c|}{ Up } \\
\hline \multicolumn{4}{|l|}{ Up } \\
\hline & ATF7IP2 & 11 & hsa-mir-548c-3p \\
\hline \multicolumn{4}{|r|}{ 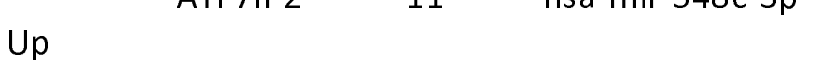 } \\
\hline \multicolumn{4}{|r|}{ 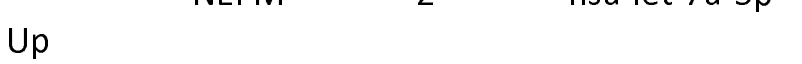 } \\
\hline \multicolumn{4}{|r|}{ (2) } \\
\hline \multicolumn{4}{|r|}{ nsa-mir- $\angle 0 D-5 p$} \\
\hline \multicolumn{4}{|l|}{ Up } \\
\hline & CCDC68 & 10 & hsa-mir-6808-5p \\
\hline \multicolumn{4}{|r|}{ 政 } \\
\hline Up & CNKSR3 & 138 & hsa-mir-2276-3p \\
\hline Up & MYZAP & 22 & hsa-mir-7153-3p \\
\hline Up & PDE6A & 34 & hsa-mir-4757-5p \\
\hline Up & ABCA8 & 2 & hsa-mir-192-5p \\
\hline Up & INHBA & 40 & hsa-mir-548i \\
\hline Up & LDLRAD2 & 7 & hsa-mir-3974 \\
\hline Up & CNTNAP3 & 4 & hsa-mir-1226-3p \\
\hline Up & C6orf141 & 22 & hsa-mir-1827 \\
\hline Up & RUNX1 & 60 & hsa-mir-1179 \\
\hline Up & TRIM71 & 104 & hsa-mir-3607-3p \\
\hline Up & & & \\
\hline & PNMT & 1 & hsa-mir-26b-5p \\
\hline & PRSS50 & 1 & hsa-mir-124-3p \\
\hline
\end{tabular}




\begin{tabular}{|c|c|c|c|}
\hline U & SLC47A1 & 52 & hsa-mir-637 \\
\hline Up & IL20RA & 1 & hsa-mir-26b-5p \\
\hline Up & LEFTY2 & 10 & hsa-mir-4267 \\
\hline Up & ITGBL1 & 18 & hsa-mir-579-3p \\
\hline Up & RHBDL3 & 4 & hsa-mir-8485 \\
\hline Up & $\mathrm{GH} 1$ & 1 & hsa-mir-7-5p \\
\hline Up & FRAS1 & 1 & hsa-mir-124-3p \\
\hline Up & СТ45A1 & 1 & hsa-mir-335-5p \\
\hline Up & FGFR3 & 9 & hsa-mir-4764-5p \\
\hline Up & EYA4 & 9 & hsa-mir-203a-3p \\
\hline Up & AMZ1 & 19 & hsa-mir-1470 \\
\hline Up & CACNA1A & 21 & hsa-mir-4734 \\
\hline Up & FAM196A & 1 & hsa-mir-335-5p \\
\hline Up & CA12 & 24 & hsa-mir-2355-3p \\
\hline Up & GAS7 & 18 & hsa-mir-384 \\
\hline Up & HPGD & 6 & hsa-mir-664a-3p \\
\hline Up & SCGB1D2 & 1 & hsa-mir-26b-5p \\
\hline Up & PLIN5 & 17 & hsa-mir-1184 \\
\hline Up & ALDOB & 2 & hsa-mir-378a-3p \\
\hline Up & GJB7 & 38 & hsa-mir-653-5p \\
\hline Up & PRR15L & 7 & hsa-mir-3679-3p \\
\hline Up & $\mathrm{IRX} 2$ & 9 & hsa-mir-6808-3p \\
\hline Up & ALDH1A3 & 31 & hsa-mir-1304-5p \\
\hline Up & EYA2 & 1 & hsa-mir-30a-5p \\
\hline up & KRT19 & 2 & hsa-mir-193b-3p \\
\hline
\end{tabular}




\begin{tabular}{|c|c|c|c|}
\hline & LYGH & 17 & hsa-mir-3188 \\
\hline Up & MYBPHL & 19 & hsa-mir-4304 \\
\hline Up & & & \\
\hline & RASGRP1 & 13 & hsa-mir-3161 \\
\hline Up & FERMT1 & 4 & hsa-mir-9-5p \\
\hline Up & ASXL3 & 28 & hsa-mir-1297 \\
\hline Up & PDZRN3 & 1 & hsa-mir-197-3p \\
\hline Up & KCNS2 & 19 & hsa-mir-888-3p \\
\hline Up & VANGL2 & 34 & hsa-mir-4733-5p \\
\hline Up & PRDM16 & 6 & hsa-mir-133a-3p \\
\hline Up & SH3BP4 & 42 & hsa-mir-663b \\
\hline Up & COL4A6 & 2 & hsa-mir-197-3p \\
\hline Up & NEUROD1 & 17 & hsa-mir-6738-3p \\
\hline Up & IL6R & 55 & hsa-mir-509-5p \\
\hline Up & FSHB & 7 & hsa-mir-3121-5p \\
\hline Up & CHRDL1 & 54 & hsa-mir-4510 \\
\hline Up & NWD1 & 75 & \\
\hline Up & CPXM2 & 14 & hsa-mir-4539 \\
\hline Up & WWC2 & 39 & hsa-mir-596 \\
\hline Up & GJB7 & 38 & hsa-mir-642a-5p \\
\hline Up & IGSF11 & 4 & hsa-mir-3163 \\
\hline Up & SCN2B & 46 & hsa-mir-4708-5p \\
\hline Up & CCDC158 & 15 & hsa-mir-1261 \\
\hline Up & ASXL3 & 28 & hsa-mir-708-3p \\
\hline Up & DLGAP2 & 8 & hsa-mir-4635 \\
\hline & AOX1 & 1 & hsa-mir-26b-5p \\
\hline
\end{tabular}




\begin{tabular}{|c|c|c|c|}
\hline $\mathrm{Jp}$ & $\mathrm{CH} 25 \mathrm{H}$ & 1 & hsa-mir-26b-5p \\
\hline Up & & 6 & \\
\hline Up & & & \\
\hline & S100B & 2 & hsa-mir-29a-3p \\
\hline Up & SLPI & 26 & hsa-mir-298 \\
\hline Up & PGR & 2 & hsa-mir-126-3p \\
\hline Up & SSTR2 & 35 & hsa-mir-6787-3p \\
\hline Up & C2orf71 & 12 & hsa-mir-3065-3p \\
\hline Up & COL13A1 & 28 & hsa-mir-940 \\
\hline Up & CACNA2D3 & 15 & hsa-mir-6503-3p \\
\hline Up & NROB1 & 1 & hsa-mir-561-3p \\
\hline Up & CALB2 & 1 & hsa-mir-335-5p \\
\hline Up & SCGB1D1 & 2 & hsa-mir-26b-5p \\
\hline Up & FGF1 & 24 & \\
\hline Up & FOXL2 & 10 & hsa-mir-520a-5p \\
\hline Up & MKX & 31 & hsa-mir-548p \\
\hline Up & TXLNB & 27 & hsa-mir-642a-5p \\
\hline Up & ERBB4 & 7 & hsa-mir-302b-3p \\
\hline Up & PCSK2 & 25 & hsa-mir-660-5p \\
\hline Up & DMKN & 18 & hsa-mir-6720-5p \\
\hline Up & TGFBR3 & 67 & hsa-mir-548aq-3p \\
\hline Up & MN1 & 17 & hsa-mir-188-3p \\
\hline Up & SLIT1 & 33 & hsa-mir-629-3p \\
\hline Up & KCNG1 & 1 & hsa-mir-324-5p \\
\hline Up & MEGF11 & 1 & hsa-mir-335-5p \\
\hline U & PLA2G4A & 35 & hsa-mir-2114-5p \\
\hline
\end{tabular}




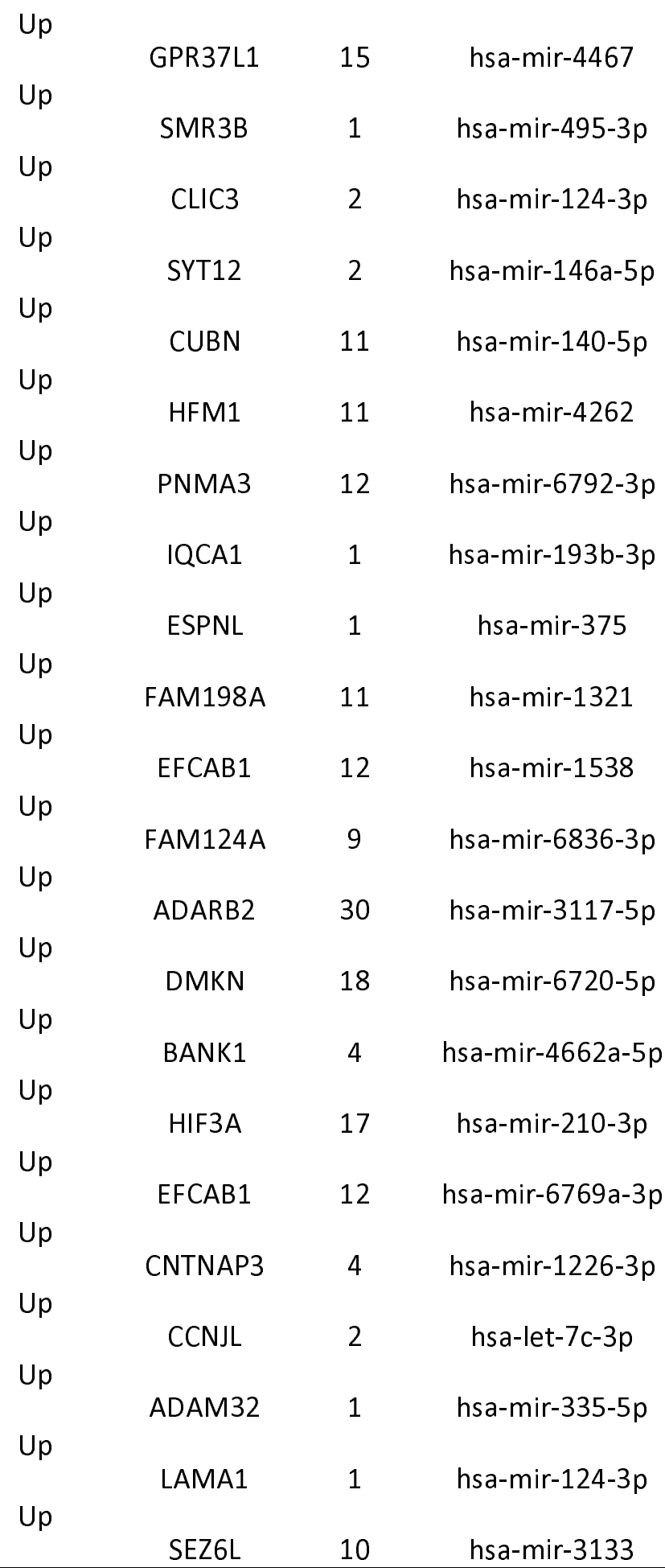

Degree - No of miRNA interact with target gene. We taken any one miRNA in table.

Table 8 TF - target gene interaction table

\begin{tabular}{llllllll}
\hline Regulation & TF & Degree & Gene & Regulation & TF & Degree & Gene \\
\hline
\end{tabular}




\begin{tabular}{|c|c|c|c|c|c|c|c|}
\hline Up & EZH2 & 73 & IRX1 & Down & CREM & 10 & ACER2 \\
\hline \multirow[t]{2}{*}{ Up } & & & & Down & & & \\
\hline & WT1 & 12 & ADAMTS18 & & TRIM28 & 14 & ARHGAP 29 \\
\hline \multirow{2}{*}{ Up } & & & & Down & & & \\
\hline & ZNF76 & 22 & ADARB2 & & GATAD1 & 11 & FU13224 \\
\hline \multirow{2}{*}{ Up } & & & & Down & & & \\
\hline & SUZ12 & 30 & CACNA2D3 & & SIN3A & 28 & ANTXR2 \\
\hline \multirow[t]{2}{*}{ Up } & & & & Down & & & \\
\hline & EBF1 & 14 & CSHL1 & & PHF8 & 31 & AKAP3 \\
\hline \multirow[t]{2}{*}{ Up } & & & & Down & & & \\
\hline & FOSL2 & 10 & AGR2 & & MXD4 & 20 & BIRC5 \\
\hline \multirow[t]{2}{*}{ Up } & & & & Down & & & \\
\hline & ZNF217 & 9 & AMZ1 & & SAP30 & 31 & HOXA4 \\
\hline \multirow[t]{2}{*}{ Up } & & & & Down & & & \\
\hline & NFIA & 11 & ALDOB & & STAT1 & 7 & CDCA5 \\
\hline \multirow[t]{2}{*}{ Up } & & & & Down & & & \\
\hline & ZNF644 & 17 & ATF7IP2 & & GLI4 & 8 & ASPDH \\
\hline \multirow{2}{*}{ Up } & & & & Down & & & \\
\hline & GATA4 & 14 & $\mathrm{CHN} 2$ & & KDM5B & 31 & ATP2A3 \\
\hline \multirow[t]{2}{*}{ Up } & & & & Down & & & \\
\hline & NR2F2 & 6 & $\mathrm{AOX} 1$ & & KDM5A & 35 & CCNF \\
\hline \multirow[t]{2}{*}{ Up } & & & & Down & & & \\
\hline & CREB1 & 14 & CNKSR3 & & ZEB1 & 26 & C3orf52 \\
\hline \multirow{2}{*}{ Up } & & & & Down & & & \\
\hline & ZBTB33 & 25 & APOD & & ATF1 & 23 & CKS2 \\
\hline \multirow{2}{*}{ Up } & & & & Down & & & \\
\hline & TFE3 & 14 & CNKSR3 & & NR2C2 & 19 & C1orf189 \\
\hline \multirow[t]{2}{*}{ Up } & & & & Down & & & \\
\hline & MBD4 & 10 & DPPA4 & & TFDP1 & 26 & CADPS2 \\
\hline \multirow[t]{2}{*}{ Up } & & & & Down & & & \\
\hline & MYBL2 & 10 & HSD17B2 & & ZNF589 & 14 & DAZAP2 \\
\hline \multirow[t]{2}{*}{ Up } & & & & Down & & & \\
\hline & ZFP64 & 15 & C6orf132 & & POLR2H & 14 & CDC45 \\
\hline \multirow[t]{2}{*}{ Up } & & & & Down & & & \\
\hline & ATF1 & 18 & CCDC68 & & ETV1 & 10 & FAM83A \\
\hline \multirow[t]{2}{*}{ Up } & GATAD2 & & & Down & & & \\
\hline & $A$ & 18 & CRYM & & SMARCA4 & 12 & CENPH \\
\hline \multirow[t]{2}{*}{ Up } & & & & Down & & & \\
\hline & RCOR2 & 13 & FGFR3 & & MAZ & 34 & BDNF \\
\hline \multirow[t]{2}{*}{ Up } & & & & Down & & & \\
\hline & BCL6 & 11 & KISS1R & & SIRT6 & 12 & HMMR \\
\hline \multirow[t]{2}{*}{ Up } & & & & Down & & & \\
\hline & FOXA3 & 15 & DMKN & & REST & 24 & CDCA5 \\
\hline Up & & & & Down & & & \\
\hline & YY1 & 9 & FABP7 & & PPARG & 17 & BIRC5 \\
\hline Up & & & & Down & & & \\
\hline & HMG20B & 16 & IL6R & & ZNF2 & 21 & BUB1 \\
\hline Up & & & & Down & & & \\
\hline & ZNF7 & 2 & PNMT & & BCL11A & 22 & CENPM \\
\hline
\end{tabular}




\begin{tabular}{|c|c|c|c|c|c|c|c|}
\hline \multirow[t]{2}{*}{ Up } & \multicolumn{7}{|c|}{ Down } \\
\hline & NFIC & 4 & FMO5 & & LEF1 & 13 & CCNG2 \\
\hline \multirow[t]{2}{*}{ Up } & & & & Down & & & \\
\hline & HNF4G & 10 & PRR15L & & INSM2 & 18 & CKS2 \\
\hline \multirow[t]{2}{*}{ Up } & & & & Down & & & \\
\hline & MBD1 & 27 & DACT2 & & BCL3 & 1 & AMIGO2 \\
\hline \multirow[t]{2}{*}{ Up } & & & & Down & & & \\
\hline & THAP1 & 6 & $\mathrm{FCN} 2$ & & NCOR1 & 19 & CLTC \\
\hline \multirow[t]{2}{*}{ Up } & & & & Down & & & \\
\hline & RARA & 6 & DMKN & & FOSL2 & 6 & MYBPC1 \\
\hline \multirow[t]{2}{*}{ Up } & & & & Down & & & \\
\hline & ETV4 & 10 & $\mathrm{ITIH} 2$ & & ZNF335 & 16 & CYP27B1 \\
\hline \multirow[t]{2}{*}{ Up } & & & & Down & & & \\
\hline & L3MBTL2 & 19 & AMOT & & TAF7 & 21 & DAZAP2 \\
\hline \multirow[t]{2}{*}{ Up } & & & & Down & & & \\
\hline & СТВР1 & 2 & ASXL3 & & CREB1 & 15 & DHX15 \\
\hline \multirow[t]{2}{*}{ Up } & & & & Down & & & \\
\hline & NONO & 3 & GRHL2 & & GABPA & 16 & CLRN2 \\
\hline \multirow[t]{2}{*}{ Up } & & & & Down & & & \\
\hline & PPARG & 19 & $\mathrm{CSH} 1$ & & L3MBTL2 & 20 & C3orf80 \\
\hline \multirow[t]{2}{*}{ Up } & & & & Down & & & \\
\hline & MAZ & 28 & BMP7 & & ZNF175 & 10 & EXD1 \\
\hline \multirow[t]{2}{*}{ Up } & & & & Down & & & \\
\hline & CBFB & 7 & FFAR3 & & ZNF639 & 16 & CRNDE \\
\hline \multirow[t]{2}{*}{ Up } & & & & Down & & & \\
\hline & SMAD4 & 14 & CLIC3 & & KLF16 & 22 & FANCA \\
\hline \multirow[t]{2}{*}{ Up } & & & & Down & & & \\
\hline & RNF2 & 12 & CCNJL & & SMAD5 & 31 & $\mathrm{CNIH} 2$ \\
\hline \multirow[t]{2}{*}{ Up } & & & & Down & & & \\
\hline & ATF4 & 4 & FRAS1 & & EED & 9 & KIF18A \\
\hline \multirow[t]{2}{*}{ Up } & & & LOC100288 & Down & & & \\
\hline & HBP1 & 16 & 911 & & NRF1 & 25 & FANCA \\
\hline \multirow[t]{2}{*}{ Up } & & & & Down & & & \\
\hline & ATF3 & 12 & C6orf141 & & SP7 & 16 & B4GALT6 \\
\hline \multirow[t]{2}{*}{ Up } & & & & Down & & & \\
\hline & KLF11 & 14 & LINC00242 & & ZNF580 & 23 & HOXA6 \\
\hline \multirow[t]{2}{*}{ Up } & & & & Down & & & \\
\hline & ZNF580 & 19 & ARMC4 & & SMARCE1 & 11 & KIF20A \\
\hline Up & & & & Down & & & \\
\hline & GATAD1 & 15 & PHYHD1 & & POLR2A & 20 & C20orf203 \\
\hline Up & & & & Down & & & \\
\hline & CTCF & 22 & ATP4A & & DMAP1 & 14 & FAM20C \\
\hline Up & & & & Down & & & \\
\hline & ZBTB26 & 16 & FAM3B & & ZNF501 & 26 & ARHGAP20 \\
\hline Up & & & & Down & & & \\
\hline & ZNF589 & 11 & CA12 & & SP3 & 11 & G2E3 \\
\hline Up & & & & Down & & & \\
\hline & PKNOX1 & 11 & $\mathrm{CSH} 2$ & & SUZ12 & 23 & CRNDE \\
\hline Up & & & & Down & & & \\
\hline & ZEB1 & 11 & AQP4 & & BCL11B & 28 & DISC1 \\
\hline
\end{tabular}


Up

ZNF263 15

Up

KLF1 $17 \quad$ C9orf152

Up

Up

Up

ZNF558 7

\section{ARMC3}

TBX21 5

\section{DMKN}

ZNF324 13

Up

Up

ZNF547 2

WNT5A

NGFR

ZNF202 2

ARMC4

Up

$\begin{array}{ll}\text { ZNF175 } & 8\end{array}$

Up

Up

ZNF239 4

ZNF121 11

Up

Up

$\begin{array}{ll}\text { TARDBP } & 7\end{array}$

SP3 19

Up

Up

PRDM2 6

BCOR 8

Up

POLR2H 2

Up

Up

CEBPA 5

TRIM24 4

Up

Up

NR2F1 13

$\begin{array}{ll}\text { ZNF384 } & 7\end{array}$

Up

Up

NCOR1 6

ART3

ASXL3

CELSR1

MKX

FAM196A

CPLX3

KISS1R

GJB7

SLC6A14

LDLRAD2

IL22RA1

ID4

KAZN

FOXL2

Up

NR4A1 11

GMEB2 2

Up

Up

THRB

11

TGIF2

19

Up

PRSS8

CTRB1

NFIX
Down

Down

Down

Down

Down

Down

GFI1B

10

FOXM1

ZNF324

17

C1orf189

ARID4B

17

MY01D

MXD3

21

DOK6

SMAD4 16

LOC339874

IRF4

13

NHLRC4

Down

PRDM10

9

MAMSTR

Down

EGR1

13

NXPH4

Down

CREB3L1

25

NUSAP1

Down

TCF7L2

1

ASPDH

Down

MTA1

15

YTHDF1

Down

SREBF1

3

PARPBP

Down

CDC5L

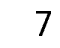

LRFN1

Down

NR2F1

18

ZSCAN12

Down

TRIM22

15

VWA1

Down

Down

IKZF1

13

TROAP

Down

ZNF202

6

TRHDE

Down

HIC1

16

TEX22

ID3

18

TCEANC2

Down

KLF6

12

TTK

Down

ZNF76

27

ZRANB2-AS1

Down

TCF7

5

SOX4

Down

DRAP1

13

SERINC2

Down

KLF9

43

UNC13A

Down

FMO5

HCFC1

15

TRIM24 
Up

\section{ZNF512 7}

Up

Up

GABPA 9

CT45A1

EVC2

ARID4B

13

PPP1R27

Up

Up

KDM1A 13

PLIN4

DMAP1 20

C1orf168

Up

Up

KLF7 21

ENTPD8

GLIS2 27

$\mathrm{KCNJ12}$

Up

ZNF146 6

Up

Up

SP1 14

OVOL3

NTF4

FXYD3

Up

ZNF488 2

BCL11A 12

KRT19

Up

ZNF341 19

COL13A1

Up

Up

BCL11B 16

EYA4

ZBTB7A 19

Up

Up

HIC1 23

ZNF501 17

Up

Up

SOX13 19

ESRRA 8

Up

INSM2 11

Up

Up

$\begin{array}{ll}\text { ZNF2 } & 17\end{array}$

ZNF610 11

Up

ETV6 1

Up

Up

ZNF394 15

ZNF18

12

Up

19

SALL1

FAM198A

MGST1

PMAIP1

ZNF24

15

Down

CEBPA

6

RASSF10

Down

Down

Down

MBD1

10

PLK1

MLX

14

ZSCAN2

Down

NFYC

11

TPX2

Down

KDM1A

9

SPC24

Down

ELF1

24

RGS5

Down

MNT

3

PARPBP

Down

PBX2

9

TLE4

HBP1

15

SLC39A5

Down

KLF8

20

RCC1

Down

KLF11

12

ZC3H12D

Down

CHD1

20

RASSF3

Down

FOXJ2

13

TCEANC2

Down

TEAD3

10

TRIM24

Down

HMGN3 16

KIF11

Down

ZNF584 9

PF4

Down

NCOA1 10

DAZAP2

Down

ZKSCAN1

8

PVT1

Down

HDAC1

12

HAUS6

Down

$\begin{array}{ll}\text { ZNF423 } & 18\end{array}$

KLHL13

Down

ZFX 23

HMMR

Down

EZH2 $\quad 34$

POU3F4

Down

EP300

4

PBK

Down

GTF2E2

28

KIAA1958

Down

DNAI2

NFRKB

12

CCNB2 
Up

$$
\text { Up }
$$

KLF8 $\quad 11$

NKX2-2

Down

$\begin{array}{cccc}\text { Up } & \text { ELF3 } & 14 & \text { BTBD17 } \\ \text { Up } & \text { DID01 } & 2 & \text { LEFTY2 }\end{array}$

Down

$\begin{array}{lll}\text { RERE } & 11 & \text { SOX9 }\end{array}$

Up

ZNF197 11

WWC2

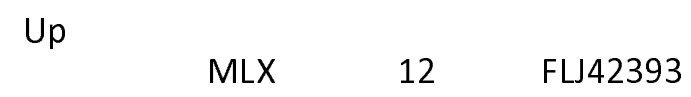

$\begin{array}{llll}\text { Up } & \text { FOSL1 } & 11 & \text { FMO5 }\end{array}$

$\begin{array}{cccc}\text { Up } & \text { ESR1 } & 3 & \text { LGI1 } \\ \text { Up } & \text { ZFP2 } & 12 & \text { STRA6 }\end{array}$

Down

HDGF $\quad 17 \quad$ POLQ

\section{Down}

JUNB 6

WRNIP1 24

LINC00309

Down

MLLT1

11

CALHM1

Down

ZFP37

23

PTP4A1

Down

TBX21

8

DISC1

Down

SMC3

6

SPC24

Down

ZBTB17 8

POU3F3

Up

Up

Up

$\begin{array}{lll}\text { POLR2A } & 7 \quad \text { LDLRAD2 }\end{array}$

Down

EHMT2 1

BDNF

Down

MYNN

21

C20orf203

NFIL3 $11 \quad$ NEURL3

KLF13

11

INHBE

Down

$\begin{array}{lll}\text { KLF16 } & 21 & \text { GH2 }\end{array}$

Up

KLF16 21

FOSL1

18

PTP4A1

Down

Up

KLF4 $16 \quad$ GADD45G

Down

CTCF

12

PKLR

REST 13

RHBDL3

ZNF644

17

MAMSTR

Up

$\begin{array}{ll}\text { RAD21 } & 17\end{array}$

RBP4

Down

Up

Up

MXD3 14

S100A1

Down

SSRP1

9

TRIM24

Down

RERE

18

PTTG2

GPR37L1

CEBPG

7

MTBP

Down

RPRML

ZHX2

11

LOC339874

Up

ZNF335 10

Down

ETS1

18

CNIH2

Up

ELF1 11

DLEC1

Down

SCRT2

6

SLC52A3

Up

Up

Up

GATA2 7

CA8

Down

GMEB2 $\quad 15$

ZSCAN12

Down

$\begin{array}{lll}\text { RXRB } & 11 \quad \text { TMEM139 }\end{array}$

MYC

5

FANCA

Down

$\begin{array}{lll}\text { DPF2 } & 6 & \text { GRHL2 }\end{array}$

SCRT1

3

STMN1

Up

NR2F6 15

PTER

Down

THRB

12

SLC25A15

Up

ZNF71 10

EFEMP1

Down

ZNF197

18

RASSF10 


\begin{tabular}{|c|c|c|c|c|c|c|c|}
\hline \multirow[t]{2}{*}{ Up } & \multicolumn{7}{|c|}{ Down } \\
\hline & ZNF101 & 7 & RNF207 & & ZBTB7A & 28 & QSOX1 \\
\hline \multirow[t]{2}{*}{ Up } & & & & Down & & & \\
\hline & JUN & 2 & C6orf141 & & MXI1 & 6 & HJURP \\
\hline \multirow[t]{2}{*}{ Up } & & & & Down & & & \\
\hline & ZNF623 & 5 & HIF3A & & ZNF394 & 24 & ZNF160 \\
\hline \multirow[t]{2}{*}{ Up } & & & & Down & & & \\
\hline & ZNF140 & 1 & C6orf141 & & ZBTB40 & 7 & G2E3 \\
\hline \multirow[t]{2}{*}{ Up } & & & & Down & & & \\
\hline & ZNF292 & 1 & C6orf141 & & $\mathrm{E} 2 \mathrm{~F} 4$ & 14 & FANCD2 \\
\hline \multirow[t]{2}{*}{ Up } & & & & Down & & & \\
\hline & ZNF264 & 2 & OVOL3 & & RNF2 & 9 & PNPLA7 \\
\hline \multirow[t]{2}{*}{ Up } & & & & Down & & & \\
\hline & CUX1 & 9 & SYT12 & & ZNF407 & 8 & CCNB2 \\
\hline \multirow[t]{2}{*}{ Up } & & & & Down & & & \\
\hline & GFI1B & 6 & TGFBR3 & & ZBTB11 & 21 & RAD51 \\
\hline \multirow[t]{2}{*}{ Up } & & & & Down & & & \\
\hline & ZNF382 & 9 & KCNG1 & & MAFG & 3 & CLTC \\
\hline \multirow[t]{2}{*}{ Up } & & & & Down & & & \\
\hline & PRDM12 & 1 & C6orf141 & & POU5F1 & 2 & BUB1 \\
\hline \multirow[t]{2}{*}{ Up } & & & & Down & & & \\
\hline & FOS & 3 & IL6R & & RCOR2 & 10 & CDKN3 \\
\hline \multirow[t]{2}{*}{ Up } & & & & Down & & & \\
\hline & PRDM10 & 14 & STRA6 & & NR4A1 & 12 & C20orf203 \\
\hline \multirow[t]{2}{*}{ Up } & & & & Down & & & \\
\hline & TEAD3 & 14 & $\mathrm{TF}$ & & TFAP4 & 16 & RFT1 \\
\hline \multirow[t]{2}{*}{ Up } & & & & Down & & & \\
\hline & IRF1 & 10 & TEPP & & ZNF101 & 11 & CADPS2 \\
\hline \multirow[t]{2}{*}{ Up } & & & & Down & & & \\
\hline & SIRT6 & 5 & CA8 & & ZNF341 & 11 & NXPH4 \\
\hline \multirow[t]{2}{*}{ Up } & & & & Down & & & \\
\hline & MIXL1 & 3 & MYBPHL & & MEF2D & 12 & $\mathrm{RCC1}$ \\
\hline \multirow[t]{2}{*}{ Up } & & & & Down & & & \\
\hline & ZBTB11 & 9 & SLC39A8 & & YY1 & 10 & KIF18A \\
\hline \multirow[t]{2}{*}{ Up } & & & & Down & & & \\
\hline & CREB3L1 & 9 & RASL11B & & EBF1 & 8 & KIR3DX1 \\
\hline \multirow[t]{2}{*}{ Up } & & & & Down & & & \\
\hline & TFDP1 & 29 & VSNL1 & & TSHZ1 & 11 & PKLR \\
\hline Up & & & & Down & & & \\
\hline & HMBOX1 & 5 & TMEM154 & & PKNOX1 & 4 & LYNX1 \\
\hline Up & & & & Down & & & \\
\hline & BHLHE40 & 7 & PPP1R27 & & WT1 & 15 & RAD21L1 \\
\hline Up & & & & Down & & & \\
\hline & $\mathrm{BACH} 1$ & 3 & PNMT & & FOXA3 & 14 & FOXM1 \\
\hline Up & & & & Down & & & \\
\hline & FOXJ2 & 7 & S100B & & PRDM1 & 10 & PRDM1 \\
\hline Up & SMARCA & & & Down & & & \\
\hline & 4 & 5 & FAM83F & & ZNF610 & 16 & SLC19A1 \\
\hline Up & & & & Down & & & \\
\hline & SIN3A & 17 & PAX6 & & ZNF18 & 17 & DISC1 \\
\hline
\end{tabular}


Up

Up

ZBTB40 6

ID3 13

Up

TRIM28 4

Up

Up

HDAC1

13

SMARCE

1

8

Up

Up

GLI4

4

SCRT2

3

Up

Up

Up

Up

Up

Up

Up

Up

Up

Up

Up

Up

Up

Up

GLIS1

15

EGR2 15

ATF2

4

HHEX 5

STAT3

5

CEBPG 14

HCFC1 4

TFAP4 14

NR2C2 10

HMGN3 8

FOXM1 7

EGR1 7

IRF4

7

DRAP1

14

Up

Up

SP7 $\quad 10$

NCOA1 3

Up

$\begin{array}{lll}\text { CREM } & 8 & \text { S100A14 }\end{array}$

Up

FBN2

CYBRD1

RUNX1

PDE6A

STRA6

RPRM

$\mathrm{CH} 25 \mathrm{H}$

Sox9

CLEC9A

TSPAN8

CLIC3

TRIM71

MFAP4

ENTPD8

NTF4

RHOD

KISS1R

NEURL3

PPP1R27
Down

Down

KLF7

20

NHLRC4

ZBTB33

15

HOXA6

Down

GLIS2

19

ZBTB33

Down

$$
\text { KLF4 }
$$

8

PRR11

Down

SUPT5H

7

EVX2

Down

ARNT

6

CDC45

Down

EGR2

13

FL13224

Down

ZNF382

6

HOXA4

Down

RFX1

7

OMP

Down

ELK1

23

ZMAT3

Down

ZNF71

17

NUF2

Down

DDX20

16

TSSC2

Down

SP1

25

LOC10026816

Down

Down

TEAD2

1

CDC45

Down

SMARCA5

13

RAD21L1

ZNF83

9

MAMSTR

Down

ZNF24

22

MTBP

Down

DNMT1

5

KIF20A

Down

GTF2A2

7

FAM83A

Down

$$
\text { E2F5 }
$$

13

DHX15

Down

IRF2

7

$\mathrm{CN} I \mathrm{H} 2$

Down

HMBOX1

\section{5}

CLTC

Down

CREB3

4

MY01D

Down

ZNF623

9

TCEANC2

Down

JUND

8

MYBPC1 
Up

$\begin{array}{cccc} & \text { LEF1 } & 3 & \text { TF } \\ \text { Up } & \text { DNMT1 } & 1 & \text { CNKSR3 }\end{array}$

Down

Up

ZNF423 $18 \quad$ PPP1R1B

Down

Up

$\begin{array}{cccc}\text { Up } & \text { TEAD1 } & 9 & \text { SPTLC3 } \\ & \text { ADNP } & 8 & \text { RNF207 }\end{array}$

Down

Up $\quad$ HES1 $\quad 5 \quad$ GADD45G

$\begin{array}{lll}\text { Up } & \text { ARNT } & 2\end{array}$

$\begin{array}{llll}\text { Up } & \text { ZFP37 } & 13 & \text { RASGRP1 }\end{array}$

Up

Up

Up

ZBTB17 4

KRT19

\section{Down}

Down

$\begin{array}{lll}\text { ZNF512 } & 7 & \text { HTR3D }\end{array}$

Down

$\begin{array}{lll}\text { ATF2 } & 6 & \text { NLGN1 }\end{array}$

$\begin{array}{lll}\text { ZFP2 } & 23 & \text { GPR161 }\end{array}$

Down

Down

$\begin{array}{lll}\text { MAFK } & 5 & \text { STMN1 }\end{array}$

$\begin{array}{lll}\text { KLF1 } & 21 & \text { ZBTB33 }\end{array}$

Down

Down

HDAC8 1

CDC45

MYNN $\quad 10 \quad$ KISS1R

ETS2

TRIM24

Down

$\begin{array}{lll}\text { ARID1B } & 7 & \text { PON3 }\end{array}$

RELA

3

PKLR

Up

$\begin{array}{lll}\text { RFXANK } & 8 & \text { PRSS8 }\end{array}$

Down

Up

ZNF143 4

COL13A1

Down

NR2F6

22

QSOX1

Up

Up

$\begin{array}{ll}\mathrm{SP} 2 & 17\end{array}$

EFCAB1

Down

HES1

$$
2
$$

KIF15

Down

ZHX1

\section{2}

TSSC2

CDC5L $\quad 5$

PTER

TSC22D4

1

CDC45

Down$$
\begin{array}{ll}
\text { ZNF16 } & 4
\end{array}
$$

RUNX1

Down

$$
\text { Up }
$$

$\begin{array}{lll}\text { PRDM1 } 4 & \text { TGFBR3 }\end{array}$

BACH1 6

ZSCAN12

Up

TRIM22 6

SULT1C4

Down

DID01 4

CYP27B1

Up

Up

$\begin{array}{lll}\text { GTF2F1 } & 11 \quad \text { SYT12 }\end{array}$

Down

$\begin{array}{ll}\text { CUX1 } & 7\end{array}$

PVT1

Down

ETV6 3

PRR11

$\begin{array}{lll}\text { TSHZ1 } & 5 & \text { KRT19P2 }\end{array}$

ARID1B

12

RACGAP1

Up

Up

RFX1 5

PGR

Down

$\begin{array}{ll}\text { ZNF207 } & 15\end{array}$

RAD51D

Down

CRISP2

IRF1

24

CENPH

Up

$\begin{array}{ll}\text { PTTG1 } 1 & 1\end{array}$

Down

$\begin{array}{lll}\text { MEF2D } & 8 & \text { PDE6A }\end{array}$

GATA4

6

TPX2

Up

SSRP1 15

TRIM71

Down

Up

NRF1

14

SALL1

Down

NFIA

9

VWA1

TGIF2

13

PLK1 
Up

\section{KDM5A 2}

Up

Up

RCOR1 6

HDAC2 5

Up

HDAC6
Up SMARCA

5

Up

Up

Up

Up

Up

Up

Up

Up

Up

Up

Up

Up

Up

Up

$$
\text { E2F5 }
$$

HDGF

6

ETV1

5

$\begin{array}{ll}\text { PYGO2 } 1 & 1\end{array}$

ELK1 6

KDM5B

11

$\begin{array}{ll}\text { NFRKB } & 7\end{array}$

MITF 2

$$
\text { PHF8 }
$$

11

ZNF639 6

SAP30

11

CREB3

6

KLF6 7

ZKSCAN1 5

Up

Up

CBX8 6

ZNF138

1

Up

$\begin{array}{ll}\text { ZNF366 } & 7\end{array}$

Up

Up

SUPT5H 5

$$
\text { USF2 }
$$

5

Up
CRYM

GRHL2

ESPNL

SSTR2

$\mathrm{GH} 2$

$\mathrm{CSH} 2$

CSHL1

DLEC1

CYBRD1

FOXL2

WWC2

LEFTY2

RASGRP1

ID4

MYBPHL LOC100288

911

SRL

VANGL2

DLK1

SOX2

DMKN

WNT5A

S100B

SYPL2

DPPA4
Down

Down

CBFB

9

PSTPIP2

TFE3

14

DISC1

Down

ZFP64

9

G2E3

Down

SIN3B

9

PTP4A1

Down

GTF2F1

17

$\mathrm{CNIH} 2$

Down

ZBTB26

11

SOX4

Down

CCNT2

8

PNPLA7

Down

TEAD1

8

NCAPG

Down

GTF2B

8

KIF11

Down

RFXANK

9

HAUS6

Down

SOX5

10

CDKN3

Down

Down

NR2F2

4

SPC24

GATAD2A

11

LYNX1

Down

THAP1

4

CLTC

Down

RFX3

6

CENPA

Down

ETV4

15

HMMR

Down

ZNF384

13

SLITRK6

Down

SP2

20

UNC13A

Down

ZNF143

11

RFC3

Down

MTA2

5

FU13224

Down

ZNF121

6

TMEM40

Down

Down

BCL6

12

YTHDF1

ATF4 7

RAD21L1

Down

USF1

3

PNPLA7

Down

PML

4

RCC1 
Up

$\begin{array}{cccc}\text { Up } & \text { EHMT2 } & 3 & \text { DRGX } \\ \text { Up } & \text { CTBP2 } & 26 & \text { LRAT } \\ \text { Up } & \text { CEBPB } & 2 & \text { PMAIP1 } \\ \text { Up } & \text { SREBF2 } & 4 & \text { MN1 }\end{array}$

Down

$\begin{array}{lll}\text { MTA1 } & 7 & \text { GPR37L1 }\end{array}$

Up $\quad$ CCNT2 $6 \quad$ FGFR3

Up JUND $\quad 4 \quad$ KRT19

Down

RAD21

16

DOK6

Down

MYBL2

SEL1L

Down

$$
\text { RARA }
$$

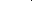

DLEU1

Down

HMG20A

6

EXD1

Down MBD4 4

SEL1L

Down HNF4G 4

CLRN2

Up

KLF13 6

KISS1R

Down

HMG20B 12

RAD51

Up

Up

MBD2

8

LGI1

Down

NFATC1 2

PVT1

Down

NONO

3

PRR11

$\begin{array}{lll}\text { ZFX } & 8 & \text { PAX6 }\end{array}$

Up

Up

WRNIP1 5

ERP27

Down

PPARGC1

$\begin{array}{lll}\text { MLLT1 } & 8 & \text { LY6H }\end{array}$

Down

Up

Up

ZNF584 4

EVC2

Down

$A$

1

CLTC

$\begin{array}{lll}\text { HLF } & 2 & \text { FAM217A }\end{array}$

Down

Up

Up

$\begin{array}{lll}\text { IKZF1 } & 8 & \text { PHYHD1 }\end{array}$

Down

TAL1

\section{5}

PVT1

Down $\quad$ KAT2A 1

$\begin{array}{lllc} & \text { TEAD4 } & 5 & \text { ITIH2 } \\ \text { Up } & \text { CEBPD } & 5 & \text { SLC39A8 }\end{array}$

Down

ARID3A 1

CLTC

$\begin{array}{lll}\text { Up } & & \\ & \text { FOXA2 } & \end{array}$

Down

GATA1 4

RFT1

Up

Up

GATA1 5

OR7A5

Down

MTA3 2

PVT1

Down

GATAD2B 3

FAM91A1

$\begin{array}{llll}\text { Up } & \text { ZNF8 } & 2 & \text { FXYD3 }\end{array}$

Up

$\begin{array}{lll}\text { MAFK } & 3 & \text { TDO2 }\end{array}$

Down

ESRRA

9

SLC39A5

$\begin{array}{lll}\text { STAT1 } & & \\ \text { FFAR3 }\end{array}$

Up

Up

$\begin{array}{lll}\text { MXD4 } & 17 & \text { SH3BP4 }\end{array}$

Down

RXRB

9

SLC25A15

Down

CTBP2

15

FOXB2

Down

ZNF366

10

TROAP

$\begin{array}{lll}\text { SMC3 } & 4 & \text { FLRT2 }\end{array}$

RUNX3

1

CXCR3

Up

GTF2A2 1

FOXL2

Down

$$
\text { SPI1 }
$$

1

CXCR3 
Up

Up $\quad$ MNT 1

Up

ZNF641 1

ZNF354C 1

Up

Up

CHD1 12

Up

ZNF592 1

Up $\quad$ ZNF207 1

Up

NFATC1 2

FOXK2 2

Up

Up

Up

GATAD2B 4

$\begin{array}{ll}\text { MYC } & 5 \\ \text { SIX4 } & 1\end{array}$

Up

Up

Up

Up

Up

Up

Up

Up

Up

Up

Up

Up

Up

Up

MTA3

2

GTF2E2 5

TBX3

2

EED

7

JUNB 5

YBX1 2

MAFF 3

GTF3C2 1

ZBTB1 3

IRF2

$$
1
$$

$$
\text { ZHX2 }
$$

3

CHD4

$$
1
$$

SMAD5

5
FRAS1

FXYD3

FXYD3

IRX2

GRHL2

GRHL2

S100A14

LY6H

RHOD

SYT12

GRHL2

S100A14

HSD11B1

PNMT

NEURL3

KAZN

$\mathrm{KCNJ} 12$

KCNJ16

KISS1R

TGFBR3

MCM3

1

KISS1R

NFIX

RNF207

KLK11
Down

Down

NFIC

$$
3
$$

ZC3H12D

Down

$$
\text { ELF3 }
$$

\section{7}

NXPH4

Down

ZNF558

8

NLGN1

Down

MAX

4

FAM83A

Down

ZNF547

6

SV2C

$\begin{array}{ll}\text { PRDM2 } & 10\end{array}$

NUF2

Down

ZNF263

17

FIBCD1

Down

ZBTB1

7

PRC1

Down

ZNF641 2

LRFN1

Down

TARDBP

SLC19A1

Down

NFIL3

9

NUSAP1

Down

HDAC2

6

ZSCAN2

Down

Down

ESR1

\section{1}

EDN1

HDAC6 6

FANCD2

Down

Down

ZNF7

\section{4}

G2E3

NFYB 2

PRR11

Down

GATA2 9

KIF21B

Down

$\begin{array}{ll}\text { PTRF } & 1\end{array}$

FAM83A

Down

CEBPD 2

FAM83A

Down

FOXA2 2

TCEANC2

Down

USF2 6

L1CAM

Down

SIX4

2

PVT1

Down

TEAD4

2

FAM83A

Down

ZNF217

2

RASSF3

Down

RCOR1

3

MTBP 
Up

TAL1 3

Up

Up

RELA 4

PTRF 2

Up

Up

ARID3A 1

Up

NFE2 2

TMEM139

$\begin{array}{lll}\text { ETS1 } & 2 & \text { ZNF385C }\end{array}$

Up

Up

AEBP2 1

OVOL3

NFE2L2 2

Up

Up

DDX20 3

EP300 1

Up

Up

MTA2 3

KAT2A

1

Up

Up

POU2F2 1

TCF7 1

Up

PML 2

Up

Up

Up

NR3C1 1

MAX 2

ZNF707 1

Up

Up

MXI1 2

NFYB 1

Up

Up

Up

Up

Up

RHOD

PDE6A

PI16

RHBDL3

PMAIP1

S100A1

PNMT

RNF207

PPP1R27

PRSS8

PRR15L

PRR15L

RHOD

RNF207

S100A1
Down

Down

Down

Down

Down

BHLHE40

13

11

ZAN

ADNP

12

TMEM40

TRHDE

POU3F3

Down

CTBP1

4

STMN1

Down

CEBPB

3

6

ZRANB2-AS1

Down

CBX8

ZNF488

1

HOXA4

Down

Down

RXRA

1

HOXA4

ZNF264

1

HOXA6

Down

ZNF16

1

HOXA6

Down

ZNF138

HOXA6

Down

ZNF707

\section{1}

INHBE

Down

NFE2L1

1

KIAA1958

Down

CHD4

\section{1}

KIF11

Down

TBP

4

NCAPG

Down

MAFF

2

KIF18A

Down

GATA3

\section{5}

SPEF2

Down

HLF

2

RAD21L1

Down

THRAP3

3

RCC1

Down

ZNF8

1

LRFN1

Down

ZNF239

2

ZNF160

Down

SIX5

1

MAMSTR

Down

RBBP5

2

MTBP

Down

RUNX1

DPF2

5

PRC1 


$\begin{array}{lccccccc}\text { Up } & \text { ZNF83 } & 1 & \text { RUNX1 } & \text { Down } & \text { MITF } & 2 & \text { ZBTB33 } \\ \text { Up } & \text { RUNX3 } & 1 & \text { RUNX1 } & \text { Down } & \text { SREBF2 } & 5 & \text { PNPLA7 } \\ \text { Up } & \text { SPI1 } & 1 & \text { RUNX1 } & \text { Down } & \text { ZNF585B } & 1 & \text { OXR1 } \\ \text { Up } & \text { SREBF1 } & 1 & \text { RUNX1 } & \text { Down } & \text { NFE2 } & 5 & \text { PF4 } \\ \text { Up } & \text { GTF2B } & 1 & \text { S100A1 } & \text { Down } & \text { MCM7 } & 1 & \text { PKLR } \\ \text { Up } & \text { PBX2 } & 1 & \text { SALL1 } & \text { Down } & \text { FOXK2 } & 1 & \text { PRR11 } \\ \text { Up } & \text { ZNF407 } & 1 & \text { SYPL2 } & \text { Down } & \text { ZNF592 } & 1 & \text { PVT1 } \\ \text { Up } & \text { HNF4A } & 1 & \text { TF } & \text { Down } & \text { ZNF140 } & 1 & \text { SLC5A10 } \\ \text { Up } & \text { RXRA } & 2 & \text { TPPP2 } & \text { Down } & \text { ZNF146 } & 2 & \text { TROAP } \\ \text { Up } & \text { TEAD2 } & 1 & \text { WWC2 } & \text { Down } & \text { TBX3 } & 2 & \text { ZC3H12D } \\ & & & \text { Down } & \text { HHEX } & 1 & \text { TPX2 }\end{array}$

Degree - No of target gene interact with TF. We taken any one target gene in table. TF transcription factors 